\title{
THE CRASHWORTHINESS BEHAVIOUR OF THIN-WALLED ALUMINIUM EXTRUDED TUBES WITH VARYING POLYGONAL CROSS SECTIONS
}

by

\author{
Anthony Rossi \\ Bachelor of Engineering, Ryerson University, 1994
}

A thesis presented to Ryerson University in partial fulfillment of the requirements for the degree of

Master of Applied Science

In the Program of

PROPERTY OF

Mechanical Engineering

GYERSON UANYERSEITY LIGRARY

Toronto, Ontario, Canada, 2004

C(Anthony Rossi) 2004 


\section{UMI Number: EC52984}

\section{All rights reserved \\ INFORMATION TO USERS}

The quality of this reproduction is dependent upon the quality of the copy submitted. Broken or indistinct print, colored or poor quality illustrations and photographs, print bleed-through, substandard margins, and improper alignment can adversely affect reproduction.

In the unlikely event that the author did not send a complete manuscript and there are missing pages, these will be noted. Also, if unauthorized copyright material had to be removed, a note will indicate the deletion.

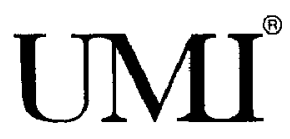

UMI Microform EC52984

Copyright 2008 by ProQuest LLC

All rights reserved. This microform edition is protected against unauthorized copying under Title 17, United States Code.

ProQuest LLC

789 East Eisenhower Parkway

P.O. Box 1346

Ann Arbor, Ml 48106-1346 
I hereby declare that I am the sole author of this thesis.

I authorize Ryerson University to lend this thesis to other institutions or individuals for the purpose of scholarly research.

Signature

I further authorize Ryerson University to reproduce this thesis by photocopying or by other means, in total or in part, at the request of other institutions or individuals for the purpose of scholarly research.

Signature 
Ryerson University requires the signatures of all persons using or photocopying this thesis. Please sign below, and give address and date. 


\title{
The Crashworthiness Behaviour of Thin-Walled Aluminium Extruded Tubes With Varying Polygonal Cross Sections
}

\author{
Anthony Rossi, Masters of Applied Science, 2004, \\ Department of Aerospace Engineering \\ Ryerson University
}

\begin{abstract}
The finite element code, LS-DYNA, was used to investigate the energy dissipation characteristics of AA6060-T4 aluminium alloy extruded tubes with varying polygonal (i.e., hexagonal and octagonal) cross sections subjected to axial impacts. The research study was conducted in two phases. The first phase comprised of validating the finite element model parameters and numerical results of thin-walled aluminium extruded square tubes with actual published experimental data. The mean dynamic axial crushing force value was predicted within $\pm 3 \%$, and in the case of the permanent axial displacement, the accuracy was within $\pm 5 \%$, when compared to the published experimental results. The second phase results of the numerical simulations pertaining to the hexagonal and octagonal tube sections were compared to the benchmark results established for the square section tubes. The numerical simulation results for the hexagonal section tube revealed a minimum average increase in the mean dynamic axial crushing force and permanent displacement parameters of $10 \%$ and $11 \%$, respectively, over the baseline square tube sections. The octagonal tube section showed a minimum average increase in the mean axial crushing force and permanent displacement parameters of $25 \%$ and $20 \%$, respectively.
\end{abstract}




\section{ACKNOWLEDGEMENTS}

I would like to give a special acknowledgement to both Dr. Z. Fawaz and Dr. K.

Behdinan. Their guidance, insight offered, and most of all patience made all the difference in the world. Their help and motivation has been a strong inspiration. I am greatly indebted to them for their work. 


\section{DEDICATION}

Where can I begin? It has been a journey taken that I would have never begun without the support of my family. A very special thank you and appreciation goes to my dear wife and kids who understood the importance of this endeavour and without their support and, most importantly, understanding, this accomplishment would never have been possible.

To my parents, who always stressed the value of an education and also for their assistance, I would like to say a very special thank you. Finally my brother, he has always been there when I needed him and not only as a sibling, but as a friend. 


\section{TABLE OF CONTENTS}

$\begin{array}{lc}\text { ABSTRACT } & \text { iv } \\ \text { ACKNOWLEDGEMENTS } & \mathbf{v} \\ \text { DEDICATION } & \mathbf{v i} \\ \text { TABLE OF CONTENTS } & \text { vii } \\ \text { LIST OF FIGURES } & \text { ix } \\ \text { LIST OF TABLES } & \mathbf{x i}\end{array}$

1.0 INTRODUCTION 1

1.1 CRASHWORTHINESS 2

1.2 IMPACT INJURY 4

1.2.1 HEAD INJURY CRITERION

$\begin{array}{llr}1.2 .2 & \text { VISCOUS (SOFT TISSUE) CRITERIA } & 8\end{array}$

1.3 OBJECTIVES OF CURRENT RESEARCH 10

2.0 STRUCTURAL IMPACT 11

2.1 INTRODUCTION 11

2.2 STRAIN RATE SENSITIVITY OF MATERIALS 13

2.2.1 CONSTITUTIVE EQUATIONS 14

2.2.2 SPLIT HOPKINSON BAR TEST (SHBT) 20

2.2.3 STRAIN RATE $\quad 24$

2.2.3.1 ALUMINIUM ALLOYS 24

2.2.3.2 STEEL ALLOYS

2.3 DYNAMIC PROGRESSIVE BUCKLING 31

2.3.1 PROGRESSIVE BUCKLING MODE 31

2.3.2 SUPER FOLDING ELEMENT METHOD 34

2.3.3 DYNAMIC AXIAL CRUSHING 41

2.3.3.1 DYNAMIC AMPLIFICATION FACTOR METHOD

2.3.3.2 STRAIN RATE SENSITIVITYEFFECT METHOD 43

2.4 DYNAMIC PLASTIC BUCKLING 47

2.4.1 DEFORMATION CHARACTERISTICS 47

2.4.2 GEOMETRICAL RELATIONS 49

2.4.3 EQUILIBRIUM EQUATIONS

2.4.4 CONSTITUTIVE EQUATIONS 53 


\subsection{NUMERICAL SIMULATION 60}

3.1 VALIDATION OF NUMERICAL RESULTS 61

3.2 VALIDATION OF COMPLEX POLYGONAL TUBE SECTIONS 64

3.3 MATERIAL PROPERTY MODEL 69

3.4 MODEL MESH ELEMENTS 73

3.4.1 BELYTSCHKO-LIN-TSAY SHELL ELEMENT CO-ROTATIONAL COORDINATES

3.4.2 VELOCITY-STRAIN DISPLACEMENT RELATIONS

3.4.3 STRESS RESULTANTS AND NODAL FORCES

$\begin{array}{lll}3.5 & \text { CONTACT-IMPACT ALGORITHM } & 82\end{array}$

3.5.1 THE PENALTY METHOD (PM) 83

3.5.1.1 THE SLAVE SEARCH

4.0 VALIDATION OF ANALYSIS 90

$4.1 \quad$ POST-BUCKLING DEFORMATION 92

5.0 RESULTS AND DISCUSSION 96

5.1 NUMERICAL SIMULATION RESULTS 96

$\begin{array}{ll}5.2 \text { DISCUSSION } & 108\end{array}$

5.3.1 REFINEMENT OF EXISTING ANALYTICAL METHODS 115

6.0 CONCLUSION 118

REFERENCES 121

APPENDLX A 


\section{LIST OF TABLES}

1.1 Impact comparison between aluminium and steel vehicles

1.2 Impact results for Audi A8 4.2

$\begin{array}{lll}2.1 & \text { Material coefficients for various materials } & 15\end{array}$

2.2 Mean crushing force of various sections 39

2.3 Material coefficients for various materials 43

3.1 Experimental square extrusion tube dynamic test specimens 62

3.2 Hexagonal tube section geometric and simulation parameters 64

3.3 Octagonal tube section geometric and simulation parameters 65

$\begin{array}{lll}3.4 & \text { Material parameters for AA6060-T4 }\end{array}$

$\begin{array}{lll}3.5 & \text { Material properties for AA6060-T4 } & 70\end{array}$

4.1 Experimental results compared vs LS-DYNA numerical 92 simulation results

5.1 LS-DYNA simulation results for square extrusion tube 103

5.2 LS-DYNA simulation results for hexagonal extrusion tube 103

5.3 LS-DYNA simulation results for octagonal extrusion tube 103

5.4 Mean axial crushing force (Pm) determined using the 104 Super Folding Element Method

5.5 Dynamic mean axial crushing force $\left(\mathrm{P}_{\mathrm{m}, \mathrm{Dyn}}\right)$ of LS-DYNA models predicted using the Dynamic Amplification Method

5.6 Dynamic mean axial crushing force $\left(\mathrm{P}_{\mathrm{m}, \text { Dyn }}\right)$ of LS-DYNA models 105 predicted using the Strain Rate Sensitivity Effect Method 
5.7 Table summarizing the dynamic mean axial crushing force determined by various methods (i.e. Experimental test, LS-DYNA, Dynamic Amplification Method, and Strain Rate Sensitivity Effect Method)

5.8 Percentage difference of mean dynamic axial force $\left(\Delta \mathbf{P}_{\mathbf{m d}}\right)$ and permanent axial displacement $\left(\Delta \delta_{p}\right)$ for hexagonal extrusion section compared to baseline square extrusion section LS-DYNA results

5.9 Percentage difference of mean dynamic axial force $\left(\Delta P_{m d}\right)$ and 109 permanent axial displacement $\left(\Delta \delta_{p}\right)$ for octagonal extrusion section compared to baseline square extrusion section LS-DYNA results

5.10 Table showing comparison between mean axial crushing force 116 predicted using the Super Element Folding Method and LS-DYNA numerical simulations 


\section{LIST OF ILLUSTRATIONS}

1.1 Audi A8 aluminium space frame structure 4

1.2(a) Whole body tolerance of impact 5

1.2(b) Influence of pulse duration on whole body tolerance of impact 6

1.3 Variation of $\mathrm{HIC}$ with impact velocity $\left(\mathrm{V}_{2}\right)$ and dimensionless 7 acceleration $(\mathrm{a} / \mathrm{g})$

2.1 Pure bending of beam with a rectangular cross-section $\mathrm{B} \times \mathrm{H}$ and made from a strain-rate sensitive material

2.2 Schematic illustration of the Split Hopkinson Bar Test (SHBT) Apparatus

2.3 Typical strain measurement history from SHBT

2.4 Typical stress-strain curve obtained using the SHBT apparatus

2.5 Stress-strain diagram for aluminium alloy generated from dynamic uniaxial compression test at various strain rates

2.6 Variation of dynamic uniaxial tensile stresses with strain rate for aluminium alloy

2.7 Stress-strain diagram for mild steel alloy generated from dynamic uniaxial compression test at various strain rates

2.8 Variation of strength with strain rate for the dynamic uniaxial behaviour of mild steel

2.9 Dynamic bending moment-rotation curves for various values of rotation rate $(\dot{\theta})$ for mild steel

2.10 Basic collapse elements: (a) Type I, (b) Type II

2.11 Paper model of square tube symmetric crushing mode Type I

2.12 Asymmetric mixed mode B crushing of a thin-walled square tube

2.13 Geometry of basic folding mechanism

2.14 Extensional elements and bending hinge lines 
2.15 Idealized axial crushing of square tube

$\begin{array}{lll}2.16 & \text { Idealized equivalent (plastic) flow stress } & 40\end{array}$

2.17 Typical energy $(E)$ and mean crush force $\left(F_{\text {mean }}\right)$ relationship 46

2.18 Permanent profile of an aluminium 6061-T6 cylindrical tube that 48 exhibits dynamic plastic buckling deformation

2.19 Permanent profile of an aluminium 6061-T6 cylindrical tube that exhibits dynamic progressive buckling deformation

2.20 Idealisation of mass $(\mathrm{M})$ with a constant velocity $\left(\mathrm{V}_{0}\right)$ impacting axially on cylindrical shell tube of length (L)

2.21 Different perspectives of cylindrical tube shell elements with loading and deformation notation

2.22 Idealized response of a cylindrical shell section that is subjected to an axial impact

3.1 LS-DYNA meshed model depicting the square tube and impact plate specimens

3.2 Tube sections used for LS-DYNA numerical simulation analysis

3.3 LS-DYNA meshed model depicting the hexagonal tube and impact plate specimens

3.4 LS-DYNA meshed model depicting the octagonal tube and impact plate specimens

3.5 Trigger profiles utilized for the finite element analysis tube sections

3.6 True stress-strain curve of AA6060-T4 alloy $t=1.8 \mathrm{~mm}$

3.7 True stress-strain curve of AA6060-T4 alloy $\mathrm{t}=2.0 \mathrm{~mm}$

3.8 True stress-strain curve of AA6060-T4 alloy $t=2.5 \mathrm{~mm}$

3.9 Element coordinate system parameters 75

3.10 Four master segments can harbour slave node $\mathbf{n}_{\mathbf{8}}$ given that 84 $\mathbf{m}_{\mathbf{s}}$ is shown the nearest master mode as shown above 
3.11 Projection of $g$ onto the master segment $\mathrm{s}_{\mathrm{i}}$

3.12 Location of contact point when $\mathrm{n}_{\mathrm{s}}$ lies above the master segment 86

4.1 Typical $2.5 \mathrm{~mm}$ thick specimen deformation characteristics 93

$\begin{array}{lll}4.2 & \text { Small amplitude buckling } & 94\end{array}$

4.3 Impact energy vs displacement curve of LS1.8-1-SQ numerical simulation 94

4.4 Impact energy vs displacement curve of LS2.5-9-SQ numerical simulation 95

5.1 Final deformation views of LS-DYNA model LS1.8-1-SQ 97

5.2 Final deformation views of LS-DYNA model LS1.8-1-HEX 97

5.3 Final deformation views of LS-DYNA model LS1.8-1-OCT 98

5.4 Typical square tube section dynamic axial crushing force vs permanent displacement (Model LS1.8-1-SQ shown) 99

5.5 Typical hexagonal tube section dynamic axial crushing force vs permanent displacement (Model LS1.8-1-HEX shown)

5.6 Typical octagonal tube section dynamic axial crushing force vs permanent displacement (Model LS1.8-1-OCT shown)

5.7 Typical square tube section permanent displacement vs impact duration (Model LS1.8-1-SQ shown)

5.8 Typical hexagonal tube section permanent displacement vs impact duration (Model LS1.8-1-HEX shown)

5.9 Typical octagonal tube section permanent displacement vs impact duration (Model LS1.8-1-OCT shown)

5.10 Typical lobe formation relevance to the dynamic axial crushing force vs the permanent displacement curve

5.11 Typical relationship showing dynamic plastic buckling effects using both the dynamic axial crushing force vs the permanent displacement curve and the permanent displacement vs impact duration curve 
A-2 Final deformation views of LS-DYNA model LS1.8-3-SQ 125

A-3 Final deformation views of LS-DYNA model LS1.8-4-SQ 126

A-4 Final deformation views of LS-DYNA model LS1.8-5-SQ 127

A-5 Final deformation views of LS-DYNA model LS1.8-6-SQ $\quad 128$

A-6 Final deformation views of LS-DYNA model LS1.8-7-SQ 129

A-7 Final deformation views of LS-DYNA model LS1.8-8-SQ 130

A-8 Final deformation views of LS-DYNA model LS1.8-9-SQ 131

A-9 Final deformation views of LS-DYNA model LS1.8-2- HEX 132

A-10 Final deformation views of LS-DYNA model LS1.8-3- HEX 133

A-11 Final deformation views of LS-DYNA model LS1.8-4- HEX 134

A-12 Final deformation views of LS-DYNA model LS1.8-5- HEX 135

A-13 Final deformation views of LS-DYNA model LS1.8-6- HEX 136

A-14 Final deformation views of LS-DYNA model LS1.8-7- HEX 137

A-15 Final deformation views of LS-DYNA model LS1.8-8- HEX 138

A-16 Final deformation views of LS-DYNA model LS1.8-9- HEX 139

A-17 Final deformation views of LS-DYNA model LS1.8-2- OCT 140

A-18 Final deformation views of LS-DYNA model LS1.8-3- OCT 141

A-19 Final deformation views of LS-DYNA model LS1.8-4- OCT 142

A-20 Final deformation views of LS-DYNA model LS1.8-5- OCT 143

A-21 Final deformation views of LS-DYNA model LS1.8-6- OCT 144

A-22 Final deformation views of LS-DYNA model LS1.8-7- OCT 145

A-23 Final deformation views of LS-DYNA model LS1.8-8- OCT 146

A-24 Final deformation views of LS-DYNA model LS1.8-9- OCT 147 



\section{Chapter 1}

\section{INTRODUCTION}

Aluminium has become the material of choice for the "light weighting" of automobile structures where the development in this application has been primarily spurred by the introduction of stricter environmental regulations to reduce "greenhouse" emissions. Aside from its obvious weight advantages over traditional automotive steel alloys, aluminium alloys also offer other significant advantages in the areas of design and manufacturing flexibility in the form of cast and extruded members to produce complex shaped components that can be used for primary structural members in automotive applications. Currently, many automobile manufacturers are establishing many innovative benchmarks in the utilization of aluminium alloys for automotive structural applications. In the event of frontal collisions, modern automotive design practice calls for the use of front longitudinal members to behave as the primary load path for the impact forces and provide energy absorption in the form of axial collapse ensuring that the resulting inertial forces transferred to the occupant's compartment are within an acceptable safety level. Essentially, extruded square and rectangular cross-sections are the prominent configurations for front longitudinal rails found currently in typical automobiles. These are critical crash energy absorbing members that deform in an axial collapsing fashion in the event of a frontal collision. The present research study shall investigate the validation and suitability of dynamic impact numerical simulations to predict the energy absorption and deformation characteristics of aluminium extruded tubes of varying polygonal sections. The study shall focus on axial impacts with the emphasis on the crash post buckling deformation response parameters consisting of the mean dynamic axial force $\left(\mathbf{P}_{\mathrm{md}}\right)$ and the permanent axial displacement $\left(\delta_{\mathrm{p}}\right)$. 


\subsection{CRASHWORTHINESS}

The term "crashworthiness" originated around 1950 being initially associated with aviation safety, but today's application is more synonymous with the automotive industry. Crashworthiness refers to the capability of a vehicle or component to provide a survivable level of protection during severe collisions.

Today, the application of crashworthiness principles are most prominent in the automotive industry and to the aviation industry on a more limited scale, since, on a statistical basis, automobiles compared to other means of transportation are the cause of the greatest number of transportation related accident victims. Crashworthiness is viewed as an additional design requirement/specification that is mandated by government authorities. In the United States and also Canada to an even greater extent, the Federal Motor Vehicle Safety Standards (FMVSS) place important performance standards for occupant protection in collisions that most directly affect the vehicle structure. Automobile crashworthiness is guided by four distinct factors: (1) limiting to tolerable levels the impact forces that are applied to occupant; (2) providing means for managing the energy of collision while at the same time maintaining adequate survival space for occupants; (3) containing the occupants within the vehicle survivable space during the collision; and (4) protecting the occupants from post-crash hazards, such as fires [1].

The layout of the various front-end components of an automobile is engineered to achieve the desirable mode of collapse and maximize energy absorption. Structural layout is important in ensuring a controlled crash through axial collapse and bending. The force-deformation response of an energy absorber under axial loading fluctuates through several peak and saddle points as the crash event progresses. The first peak characterizes the maximum crush load of the member and can be as high as twice the mean crush load. The axial component is usually triggered through weakening the first section by the forming of small indentations (also known as triggers) at the appropriate folding distance, in order to soften the maximum crash load. This triggering is done to initiate the crushing of the component, secure a sequential collapse, and protect other 
system components from premature failure. The maximum load, despite its high value, does not significantly affect the total energy absorbed by the component, which is computed by integrating the force-deformation response. This is due to the very brief, few milliseconds duration of the peak load. Subsequent peaks are much lower than the maximum peak load, and are generally characteristic of the corner load on the section and thus for crash design analysis, the dynamic mean force is the primary parameter used for comparative and validation analysis.

Steel has been the primary material in the automotive industry for decades, but due to environmental issues that are forcing manufacturers to reduce overall fuel consumption rates and greenhouse gas emissions of new vehicles, they have had to turn to alternate lightweight materials. As a result, aluminium is gaining acceptance within the automotive industry due to the potential weight savings ranging anywhere between $25 \%$ to $40 \%$ when compared to the traditional steel structures of current vehicles [2]. In addition to general weight reductions, aluminium provides improved crash performance due to the higher crash energy absorption capacity over that of steel when both are compared on a weight specific basis. Aluminium side impact beams of various designs are used in numerous car models, as are bumper beams. In particular, aluminium extrusions and thin-walled die castings are gaining vast popularity with the advantage being that there are little geometrical limitations with respect to the complexity of the section and that they offer simultaneous applications as joining elements. Thus the proper use of extruded or die-cast products enables the development of new, innovative structural design solutions, and, as a result, significant weight and cost savings by parts integration. Therefore, the use of aluminium extrusions and castings is altering the manufacturing process of automobiles, giving way to both bonded and bolted space frame structures, that are not too different from traditional aluminium aerospace structures.

For structural applications, aluminium-magnesium-silicon alloys (AlMgSi) such as AA6014, AA6060, and AA6061 have been the popular choices due to their excellent crash energy absorption and formability characteristics [2]. 
Figure 1.1 shows a typical aluminium alloy space frame structure. The ever increasing application of aluminium in automobile structures has led to the incorporation of new non-traditional manufacturing processes such as castings and extrusions which are quite evident in the figure.

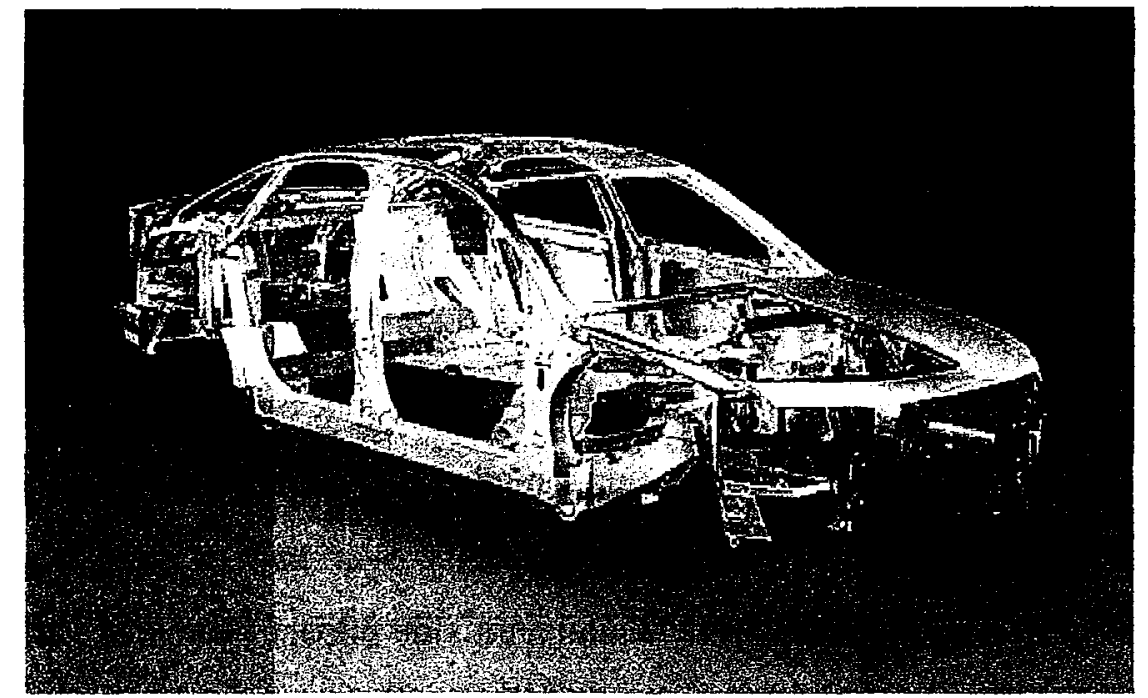

Figure I.I - Audi A8 aluminium space frame structure [2].

\subsection{IMPACT INJURY}

In the design of passenger transportation systems, the definition of structural crashworthiness can be extended to ensuring that the occupants can tolerate a certain level of impact severity that minimises their risk of serious injury. Many studies have been conducted over the years to gather valuable data regarding the behaviour of the human anatomy from the effects of impacts. 
Some typical values of whole body accelerations and their relation to severity of injury are presented in the Figures 1.2(a) and 1.2 (b). As shown in Figure 1.2(a), the extreme acceleration survival limit for the typical adult is $200 \mathrm{Gs}$, where the chances of survival actually diminish significantly beyond 20 Gs.

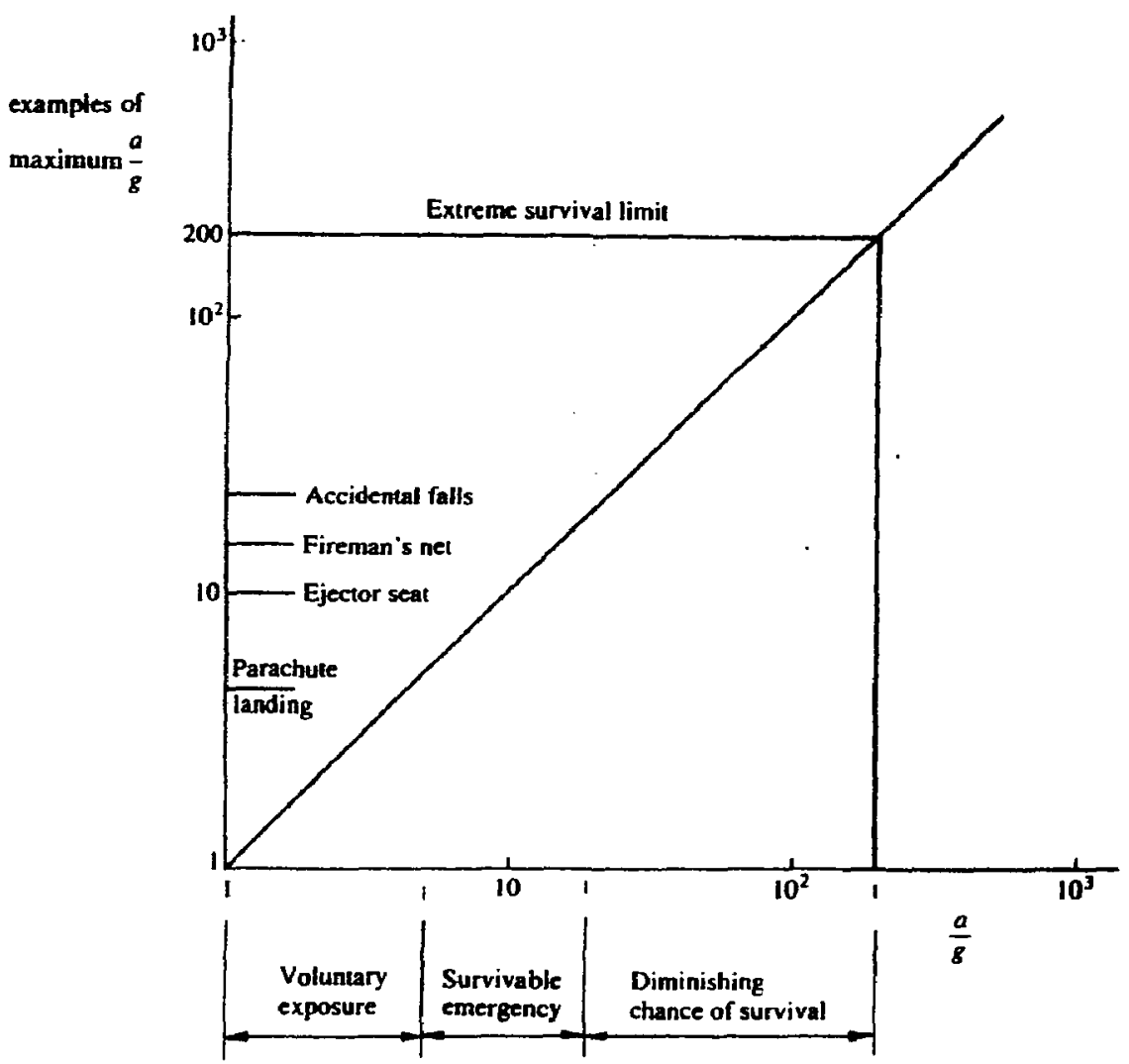

Figure 1.2(a) - Whole body tolerance of impact [3]. 


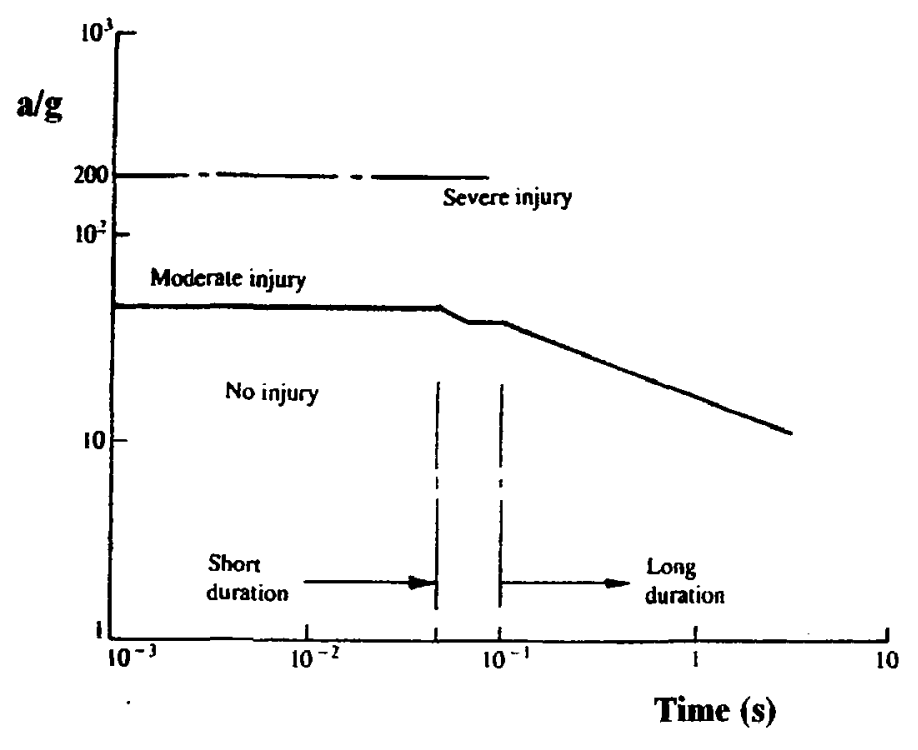

Figure 1.2 (b)- Influence of pulse duration on whole-body tolerance of impact [1].

As can be seen, the upper limit for a typical adult corresponds to an acceleration of approximately $200 \mathrm{~g}$, but this acceleration (deceleration) can only be sustained for a short duration of time ( $<0.07$ seconds). Thus, the degree of injury due to impacts is not only determined by the rate of acceleration/deceleration, but also the duration of the sustained rate of acceleration/deceleration.

\subsubsection{HEAD INJURY CRITERION}

Head injuries are responsible for a significant loss of life in transportation accidents and for this very reason, have received quite a significant amount of attention from both the medical and transportation communities. As a result of the research conducted, the Head Injury Criterion (HIC) was formulated to assess the severity of head injuries when head contact occurs. The US regulations, FMVSS 208 and FVMSS 214, on occupant crash protection specify that values of HIC greater than or equal to 1000 (HIC $\geq 1000)$ are life threatening (fatal) [1]. The expression for the HIC is defined as: 


$$
H I C=\left(T_{2}-T_{1}\right)\left[\frac{\int_{T_{1}}^{T_{2}} A_{v} d t}{T_{2}-T_{1}}\right]^{2.5}
$$

where:

$T_{1}=$ initial time of head contact

$T_{2}=$ final time of head contact

$A_{v}=$ dimensionless acceleration $=a_{v} / g$

$\mathbf{a}_{\mathbf{v}}=$ average head acceleration / deceleration

$\mathrm{g}=$ gravitational constant

It is important to note that the validity of the HIC is for pulse durations that vary between 2.5 milliseconds and 50 milliseconds $(2.5 \mathrm{~ms} \leq \mathrm{T} \leq 50 \mathrm{~ms})$. The data presented in Figure 1.3 on the next page illustrates the variation of the HIC with the impact velocity and accelerations that occur as a result. Therefore, the importance of a vehicle structure to exhibit good energy absorbing characteristics with the resulting impact accelerations being maintained below the HIC upper threshold limit to minimise the risk of serious head injuries is paramount.

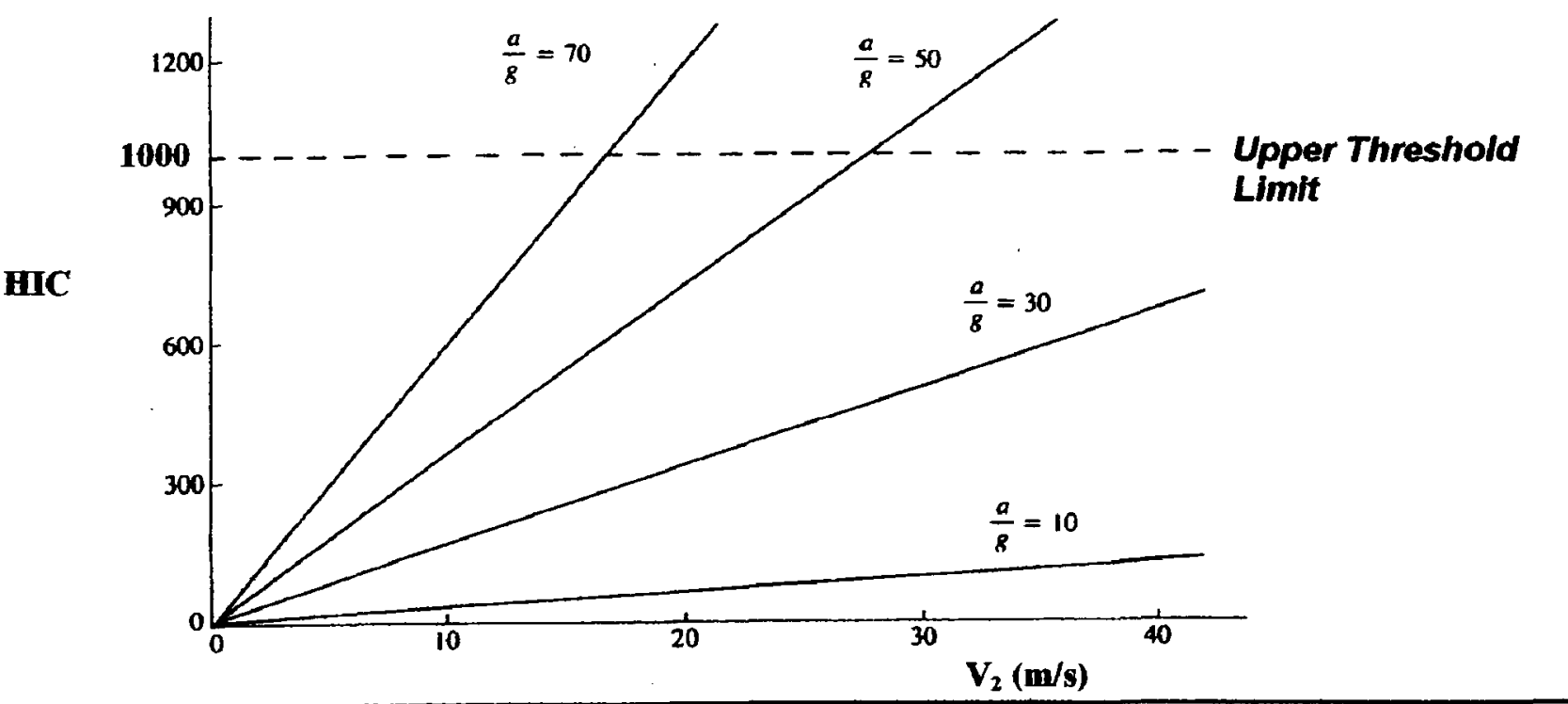

Figure 1.3 - Variation of $\mathrm{HIC}$ with impact velocity $\left(\mathrm{V}_{2}\right)$ and dimensionless acceleration $(\mathrm{a} / \mathrm{g})$ [3]. 


\subsubsection{VISCOUS (SOFT TISSUE) CRITERIA}

Like head injuries, chest injuries can be quite severe and more than often can also be fatal. A particularly common chest injury occurs to one out of every six persons who die in a car crash. The cause of death is attributed to a circumferential tear of the aorta, the largest artery in the body that carries the entire blood output of the left side of the heart. More than half of the time it tears at exactly the same spot where that restraint use doesn't seem to matter and that one need not necessarily break anything else in the chest for this artery to tear.

The Viscous (soft tissue) Criteria (VC) assists in quantifying the severity damage to the chest and its internal organs [3].

$$
V(t) C(t)=\operatorname{MAX}\left[\left(\frac{D_{t}}{0.14}\right)\left(\frac{8\left(D_{t 1}-D_{t 2}\right)}{12(t 1-t 2)}\right)\right]
$$

$V(t)$ is the sternum deflection velocity at time $(t)$ and is computed as:

$$
V(t)=\frac{8\left(D_{t 1}-D_{t 2}\right)}{12(t 1-t 2)}
$$

where:

$D_{t 1}=$ rib deflection at time $t l$

$\mathrm{D}_{12}=$ rib deflection at time $\mathrm{t} 2$

$\mathrm{t} 1$ = time interval at impact initiation

$\mathrm{t} 2=$ time interval at impact termination

$C(t)$ is the compression at time (t) and is given by:

$$
C(t)=\frac{D_{t}}{0.14}
$$

where:

$D_{t}=D_{t}-D_{t 1}$ 
To minimize the severity of impacts, the product of $V(t) C(t)$ should be equal to or less than 1 meter/second. Because of the time dependency of the Viscous Criteria, its two components $V(t)$ and $C(t)$ are computed at each time interval, $\Delta t$. Since the product $V(t)$ $\mathbf{C}(\mathrm{t})$ is computed at each time step $(\Delta t)$, the Viscous Criteria is the maximum value of all the products.

The data presented in Tables $1.1 \& 1.2$ not only illustrate the use of the HIC as one of the important parameters in effectively assessing the crashworthiness of vehicles, but also the crash response pertaining to other anatomical areas are measured. In relation to the Viscous Criteria, both the chest acceleration and displacement are measured.

Table 1.1- Impact comparison between aluminium and steel vehicles [4].

\begin{tabular}{|c|ccc|}
\hline \multicolumn{4}{|c|}{ Frontal Barrier Crash Results for Ford Aluminium and Steel Vehicles. } \\
\hline & Aluminium & Steel & FVMSS 208 Req. \\
\hline Dynamic Crash (in) & 30.8 & 28.4 & -100 \\
Head Injury Criteria & 549 & 524 & 60 \\
Chest Acceleration (g) & 37 & 53 & 3.0 \\
Chest Displacement (in) & 1.4 & 1.4 & -- \\
Torso Belt Loads (lbs) & 1219 & 1686 & 2250 \\
Left Femur Loads (lbs) & 697 & 1644 & 2250 \\
Right Femur Load (lbs) & 906 & 1092 & \\
\hline
\end{tabular}

Table 1.2- Impact results for Audi A8 4.2 [4].

\begin{tabular}{|c|ccc|}
\hline & Frontal Barrier Crash Results for Audi A8 4.2 & \\
\hline & Driver & Passenger & Low Risk Values \\
\hline Head Injury Criteria & 531 & 424 & 1000 \\
Head Acceleration (g) & 61 & 54 & 90 \\
Chest Acceleration (g) & 56 & 57 & 60 \\
Pelvis Acceleration (g) & 61 & 66 & 75 \\
Force on Thighs (lbs) & 530 & 587 & 1349 \\
Impact Duration (ms) & 15 & $\mathbf{2 3}$ & - \\
\hline
\end{tabular}




\subsection{OBJECTIVES OF CURRENT RESEARCH}

The focus of the present study is to examine in detail how the optimization of tube sections can improve their axial impact energy absorption characteristics. The optimization shall be carried out while maintaining a constant material section area between the tube configurations which will facilitate the ability to make comparisons.

Particular emphasis shall be placed on the understanding of the collapsing mechanisms that come into effect during an impact event. LS-DYNA shall be the computational tool for the finite element analysis in this research study and as such will be used for validation applications. The first phase of the research study consists of formulating a rigorous model to validate and benchmark the mean dynamic axial crushing force and resulting permanent axial displacement parameters pertaining to experimental impact test data compiled by Langseth and Hopperstad $[5,6,7]$ for aluminium thin-walled extruded square tubes. The second phase of the research study consists of using the validated LSDYNA model to analyze more complex hexagonal and octagonal section tubes and compare the numerical results against the benchmark values established from the first phase of the research study. The post-buckling deformation characteristics of the complex tube sections shall be assessed based on existing impact theory with the intent of promoting a better understanding of the complex interactions occurring during the plastic deformation phases. Finally, the computational results will be combined with current analytical methods $[8,9]$ to provide a more refined analytical means to improve the overall accuracy in predicting the mean dynamic axial crushing force in applications pertaining to preliminary design and parametric studies involving energy absorbing members. 


\section{Chapter 2}

\section{STRUCTURAL IMPACT}

\subsection{INTRODUCTION}

A literature review was conducted in preparation for the research study in order to assess the current research activity in the diverse field of impact mechanics. Although the static plastic behaviour of structures was first studied in the late 19th century [10], more systematic investigations on the dynamic plastic behaviour of structures is much more recent. There are key researchers, such as W. Abramowicz, N. Jones, and T. Wierzbicki, who most certainly can be considered pioneers in this field with their contributions of the past four decades significantly enhancing our understanding of this complex phenomenon $[8,9,11,12]$.

The research work of $\mathrm{W}$. Abramowicz, N. Jones, and T. Wierzbicki has centred on the crushing mechanics of thin-walled structures with extensive experimental analysis being conducted. There exist a plethora of technical publications that don their names, but it seems that most of their work has centred around structures composed of circular and square tubes. The significant contributions of Abramowicz and Wierzbicki in this field include their analytical method known as the Super Folding Element Method that allows the estimation of the mean dynamic crush force for an axially impacted column member. 
The Super Folding Element Method also includes a noteworthy parameter proposed by Abramowicz and Wierzbicki which is known as the plastic (energy equivalent) flow stress which compensates for the strain hardening effects found in certain engineering materials.

The properties of many materials under dynamic loading conditions are different from the corresponding static values. In particular, the stress-strain relations are sensitive to the speed of a test, a phenomenon that is known as strain rate sensitivity, or viscoplasticity. This aspect of material science spurred by research into structural impacts has seen significant contributions over the past half century with pioneers such as H. Kolsky. In the 1940s, Kolsky conducted extensive research involving the effects of high rates of strain on the mechanical properties of metals $[13,14,15,16]$.

As of the mid 1990s, the research team of M. Langseth and O. Hopperstad from the Norwegian University of Science and Technology had began to expand on the work of Abramowicz and Wierzbicki by performing extensive experimental work that included both static and dynamic crushing of thin-walled circular and square sections. In addition, they have also conducted both static and dynamic crushing of circular and square sections filled with foam. Their technical publications contain an extensive array of experimental data $[6,7]$.

After reviewing many technical publications, it was observed that the work (i.e. analytical and experimental) conducted in the past had primarily focused on simple section columns (i.e. circular and square), and any technical literature with respect to the crashworthiness behaviour of complex polygonal tube sections is significantly lacking. It is this lack of significant technical information pertaining to the crashworthiness behaviour of more complex tube sections that has motivated this research study in a hope that further work can be inspired. 


\subsection{STRAIN RATE SENSITIVETY OF MATERIALS}

The design of structures is based on material data obtained from experimental means and is normally presented in the form of a stress-strain diagram. The majority of available tabulated material properties data were gathered by deforming the specimen very slowly (quasi-static) with the average strain rate used in obtaining the stress-strain diagram being in the order of 0.001 inch / inch per second or less. Many researchers have known that the behaviour of a given material differs at higher rates of strain such as what occurs during impact events where significant structural deformation may occur over a very short period of time. Thus, the material behaviour obtained for quasi-static conditions becomes irrelevant for applications where high strain rates are encountered and as a result the material is deemed to be strain rate sensitive in most cases. Material strain rate sensitivity, or viscoplasticity, implies that the plastic flow of some materials is sensitive to strain rate which tends to primarily manifest itself as a strengthening effect in structures. In most cases, experimental testing is still the only means to obtain accurate data for materials at high strain rates. 


\subsubsection{CONSTITUTIVE EQUATIONS}

Many decades of research into the strain rate sensitive behaviour of materials has produced different constitutive equations with varying results in their ability to predict material behaviour. In order to generate the various coefficients used in the constitutive equations, careful experimental work is required, but still there exists considerable uncertainty and lack of reliable data even for some common materials. In addition, for the complex interaction of combined loading such as in the case of biaxial loading, there exists insufficient data and more research is still required to formulate practical constitutive equations.

The unit of measurement for strain rate is normally depicted as $1 /$ second $\left(\mathrm{s}^{-1}\right)$ but in some literature it is also presented as inch/inch/second. In any case, the unit derivation is shown below in the expression for the dynamic axial strain rate:

$$
\dot{\varepsilon}=\frac{\varepsilon}{t}=\frac{\delta / L}{t}=\frac{V}{L}=\frac{L}{L t}=\frac{1}{t}
$$

where:

$\dot{\varepsilon}=$ uniaxial plastic strain rate

$\varepsilon=$ strain

$\mathbf{L}=$ initial length of member (prior to deformation)

$\delta=$ change in length of member as a result of deformation

$\mathrm{V}=$ impact velocity of member

For uniaxial dynamic loading, Cowper and Symonds [10] proposed a constitutive equation that yields reasonable estimates of the strain-rate sensitive behaviour of various metallic materials:

For $\sigma_{0}^{\prime} \geq \sigma_{0}$,

$$
\dot{\varepsilon}=D\left(\frac{\sigma_{0}^{\prime}}{\sigma_{0}}-1\right)^{q}
$$


where:

$\dot{\varepsilon}=$ uniaxial plastic strain rate

$\sigma_{0}^{\prime}=$ dynamic flow stress

$\sigma_{0}=$ static uniaxial flow stress

D $=$ material constant

$q=$ material constant

The flow stress $\sigma_{0}$ is best approximated according to Wierzbicki and Abramowicz by an energy equivalent stress in the form of $[11,12]$ :

$$
\sigma_{0}=\sqrt{\sigma_{\text {Yield }} \sigma_{\text {Uh }}}
$$

where $\sigma_{Y i e l d}$ and $\sigma_{U t}$ are the material yield and ultimate tensile stresses. The flow stress in simplistic terms can be described as the stress that gives rise to a deformation energy equal to the dissipated energy, where the latter for a specific strain is given by the area under the stress-strain curve.

Equation (2.1) can be rearranged in the form:

$$
\frac{\sigma_{0}^{\prime}}{\sigma_{0}}=1+\left(\frac{\dot{\varepsilon}}{D}\right)^{1 / q}
$$

Table 2.1 - Material coefficients for various materials [10].

\begin{tabular}{|c|c|c|}
\hline Material & $\mathbf{D}\left(\mathbf{s}^{-1}\right)$ & $\mathbf{q}$ \\
\hline Mild Steel & 40.4 & 5 \\
\hline Aluminium alloy & 6500 & 4 \\
\hline Titanium (Ti 50A) & 120 & 9 \\
\hline Stainless Steel 304 & 100 & 10 \\
\hline
\end{tabular}

The material constants $\mathrm{D}$ and $\mathrm{q}$ are determined from dynamic uniaxial, or pure shear tests on the material. 
The Cowper-Symonds constitutive equation can also be expressed in the following form for specimens with triaxial stress states:

$$
\frac{\sigma_{e}^{\prime}}{\sigma_{0}}=1+\left(\frac{\dot{\varepsilon}_{e}}{D}\right)^{1 / q}
$$

Using the octahedral stress equations, $\sigma_{e}^{\prime}$ is the equivalent or effective dynamic flow stress:

$$
\sigma_{e}^{\prime}=\frac{\sqrt{\left(\sigma_{x}^{\prime}-\sigma_{y}^{\prime}\right)^{2}+\left(\sigma_{y}^{\prime}-\sigma_{z}^{\prime}\right)^{2}+\left(\sigma_{z}^{\prime}-\sigma_{x}^{\prime}\right)^{2}+6\left(\tau_{x y}^{\prime 2}+\tau^{\prime 2}{ }_{y z}^{2}+\tau_{z x}^{\prime 2}\right)}}{\sqrt{2}}
$$

where:

$\sigma_{x}^{\prime}=\sigma_{y}^{\prime}=\sigma_{z}^{\prime}=$ normal stress components

$\tau_{\mathrm{xy}}^{\prime}=\tau_{\mathrm{yz}}^{\prime}=\tau_{\mathrm{zx}}^{\prime}=$ shear stress components

and $\dot{\varepsilon}_{\mathrm{e}}$ is the associated equivalent or effective strain rate:

$$
\dot{\varepsilon}_{\theta}=\frac{\left.\sqrt{2\left(\left(\dot{\varepsilon}_{x}-\dot{\varepsilon}_{y}\right)^{2}+\left(\dot{\varepsilon}_{y}-\dot{\varepsilon}_{z}\right)^{2}+\left(\dot{\varepsilon}_{z}-\dot{\varepsilon}_{x}\right)^{2}+6\left(\dot{\varepsilon}_{x y}^{2}+\dot{\varepsilon}_{y z}^{2}+\dot{\varepsilon}_{z x}^{2}\right)\right.}\right)}{3}
$$

where:

$\dot{\varepsilon}_{\mathrm{x}}=\dot{\varepsilon}_{\mathrm{y}}=\dot{\varepsilon}_{\mathrm{z}}=$ normal strain components

$\dot{\varepsilon}_{\mathrm{xy}}=\dot{\varepsilon}_{\mathrm{yz}}=\dot{\varepsilon}_{\mathrm{zx}}=$ shear strain components

In the uniaxial case, when all the stress components are zero except $\sigma_{x}^{\prime}$, the effective dynamic flow stress reduces to $\sigma_{\mathrm{e}}^{\prime}=\sigma_{\mathrm{x}}^{\prime}$ and the effective strain reduces to $\dot{\varepsilon}_{\mathrm{e}}=\dot{\varepsilon}_{\mathrm{x}}$. In 
the case of pure shear in the $x-y$ plane where $\tau_{x y}^{\prime} \neq 0, \dot{\varepsilon}_{x y} \neq 0$, and the remaining stress and strain rate components are zero, Equations (2.5a) and (2.5b) reduce to:

$$
\sigma_{\mathrm{e}}^{\prime}=\sqrt{3} \tau_{\mathrm{xy}}^{\prime} \quad \text { and } \quad \dot{\varepsilon}_{\mathrm{e}}=\frac{2 \dot{\varepsilon}_{\mathrm{xy}}}{\sqrt{3}}
$$

For the beam presented below which has a rectangular section with width $B$ and depth $H$, a constitutive equation for the strain-rate sensitive behaviour of a material subjected to a pure bending moment is developed based on the Cowper-Symonds constitutive equation [13].

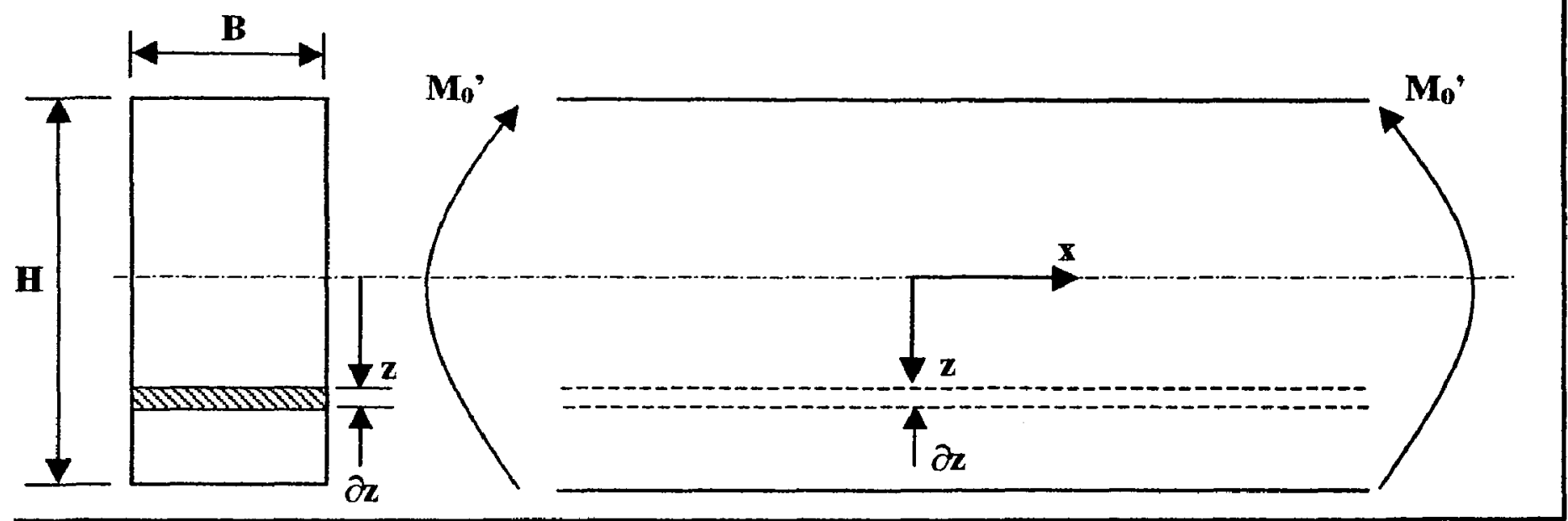

Figure 2.1 - Pure bending of beam with a rectangular cross-section $\mathrm{B} \times \mathrm{H}$ and made from a strain-rate sensitive material [10].

Assuming the material has the same behaviour in tension and compression (in essence the stress-strain curve is similar for both load cases and, by default, the area under the curve being equal), the dynamic bending moment can be expressed as:

$$
M_{0}^{\prime}=2 \int_{0}^{\frac{H}{2}} \sigma_{x}^{\prime} z B d z
$$


and the dynamic flow stress as:

$$
\sigma_{x}^{\prime}=\sigma_{0}\left[1+\left(\frac{\dot{\varepsilon}_{x}}{D}\right)^{1 / q}\right]
$$

where $\dot{\varepsilon}_{\mathrm{x}}$ is the axial strain rate at a distance $z$ from the neutral axis of the beam. Recalling that simple beam bending theory stipulates that plane cross-sections remain plane, the axial strain can be expressed as:

$$
\varepsilon_{\mathrm{X}}=\mathrm{zK}
$$

where $K$ is the change of curvature of the neutral axis. Similarly, the dynamic axial strain rate at a distance $z$ from the neutral axis of the beam can be expressed as:

$$
\dot{\varepsilon}_{\mathrm{x}}=\mathrm{z} \dot{\mathrm{K}}
$$

Substituting equations (2.8) and (2.10) into (2.7) yields:

$$
\begin{aligned}
& M_{0}=2 B \int_{0}^{\frac{H}{2}} \sigma_{0}\left[1+\left(\frac{z \dot{K}}{D}\right)^{1 / q}\right] z d z \\
& \text { or } \quad M_{0}^{\prime}=2 B \sigma_{0}\left[\frac{z^{2}}{2}+\frac{z^{2+1 / q}}{2+1 / q}\left(\frac{\dot{\kappa}}{D}\right)^{1 / q}\right]_{0}^{\frac{H}{2}}
\end{aligned}
$$

An expression for the ratio of dynamic bending moment $\left(M_{0}^{\prime}\right)$ and static fully plastic bending moment $\left(\mathrm{M}_{0}\right)$ can be expressed as:

$$
\frac{M_{0}^{\prime}}{M_{0}}=1+\frac{2 q}{2 q+1}\left(\frac{H \dot{K}}{2 D}\right)^{\frac{1}{q}}
$$


where:

$\mathrm{M}_{0}=$ fully plastic bending moment $=\frac{\sigma_{0} \mathrm{~B} \mathrm{H}^{2}}{4}$
$\frac{H \dot{\mathrm{K}}}{2}=$ maximum strain rate in the beam with occurs at the top and bottom fibres of the beam.

Equation (2.13) predicts the behaviour of a beam with a rectangular cross-section when subjected to a dynamic pure bending moment and is based on the Cowper-Symonds constitutive equation. With the constants $\sigma_{0}, D$, and $q$ evaluated from a uniaxial tensile or compressive test and substituted into equation (2.13), a relative reasonable engineering estimate of the strain-sensitive moment curvature characteristics of a material can be obtained.

For instance, in the case of mild steel and referring to Table 2.1 , the values for $\mathrm{D}$ and $\mathrm{q}$ are $40.4 \mathrm{~s}^{-1}$ and 5, respectively. Substituting the values for $\mathrm{D}$ and $\mathrm{q}$ into equation (2.13) yields

$$
\frac{\mathrm{M}_{0}^{\prime}}{\mathrm{M}_{0}}=1+\frac{10}{11}\left(\frac{\mathrm{H} \dot{\kappa}}{80.8}\right)^{0.2}
$$

where $\dot{\boldsymbol{K}}=\frac{\dot{\theta}}{\mathrm{L}}$ when $\dot{\theta}$ is the angular change across a beam of length $\mathrm{L}$ subjected to a pure bending moment $\mathrm{M}_{0}^{\prime}$. Aspden and Campbell showed good agreement when comparing their experimental results with the constitutive Equation (2.14) for the case of mild steel [13]. 


\subsubsection{SPLIT HOPKINSON BAR TEST (SHBT)}

The Split Hopkinson Bar Test (SHBT) apparatus is currently the most widely used device to test materials at high strain rates $[14,15]$. Constant strain rate tests can be performed at strain rates approaching $10^{4} \mathrm{~s}^{-1}$ quite easily.

In the Split Hopkinson Bar Test, a short cylindrical specimen is sandwiched between two long elastic bars as in Figure 2.2. The bars are generally made of high strength Maraging steel with diameters less than 0.75 inches and a length near five feet. The specimen dimensions are normally 0.25 inches in diameter and 0.25 to 0.75 inches in length. The ends of the pressure bars and specimen are machined flat to enforce desired boundary conditions. Typically, a projectile (striker bar) is fired into the end of the input bar generating a compressive stress pulse. Immediately following impact, this pulse travels along the bar towards the input bar-specimen interface at which the pulse is partially reflected into the input bar and partially transmitted through the specimen and into the output bar. The reflected pulse is reflected as a wave in tension, whereas the transmitted pulse remains in compression. The strain histories in the two pressure bars are recorded by strain gauges 1 and 2 as shown in Figure 2.2 .

So long as the pressures in the bars remain under their elastic limits, specimen stress, strain, and strain rate may be calculated from the recorded strain histories. Under certain deformation conditions, only two important strain pulses need be identified. These are the reflected pulse and the pulse transmitted through the specimen as shown in Figure 2.3. Kolsky developed the following relation for calculating the specimen stress $[16,17]$ :

$$
\sigma_{s}(t)=E \frac{A_{v}}{A} \varepsilon_{T}(t)
$$

where $E$ is the output pressure bar's elastic modulus, $A_{o}$ is the output bars cross sectional area, $A$ is the sample's cross sectional area, and $\varepsilon_{T}(t)$ is the transmitted strain history. Specimen strain rate may be calculated from 


$$
\frac{d \varepsilon_{S}(t)}{d t}=-\frac{2 C_{0}}{L} \varepsilon_{R}(t)
$$

where $\varepsilon_{R}(t)$ is the reflected input bar strain history, $L$ is the specimen length prior to impact, and $C_{o}$ is the infinite wavelength wave velocity in the input pressure bar, calculated from elementary vibrations as

$$
C_{0}=\sqrt{\frac{E}{\rho}}
$$

where $E$ and $\rho$ are the bars elastic modulus and density, respectively. Equation (2.16) can be integrated in time to yield the specimens strain, given by Equation (2.18).

$$
\varepsilon_{S}(t)=-\frac{2 C_{0}}{L} \int_{0}^{t} \varepsilon_{R}(t) d t
$$

Though these equations have been around since the late 1940's, it has only been with the advent of high-speed computers where Fast Fourier Transform analysis and other numerical procedures can be completed quickly that their potential has fully been realized. The recorded strain histories must first be corrected for dispersion, then examined such that the precise beginning and ending of the reflected and transmitted pulses may be identified. Many testing conditions greatly affect the implementation and effectiveness of the above equations. For instance the length of the impact pulse determines how much the specimen may be deformed. As mentioned, the waves are dispersed, which in effect smears the waveforms. In addition, the fundamental assumption that the specimen deforms uniformly can only be achieved by proper lubrication of the specimen-pressure bar interface and the proper selection of dimensions for the specimen. By carefully addressing these issues, dynamic stress-strain relations can be found for a broad range of materials using the previously defined equations. 


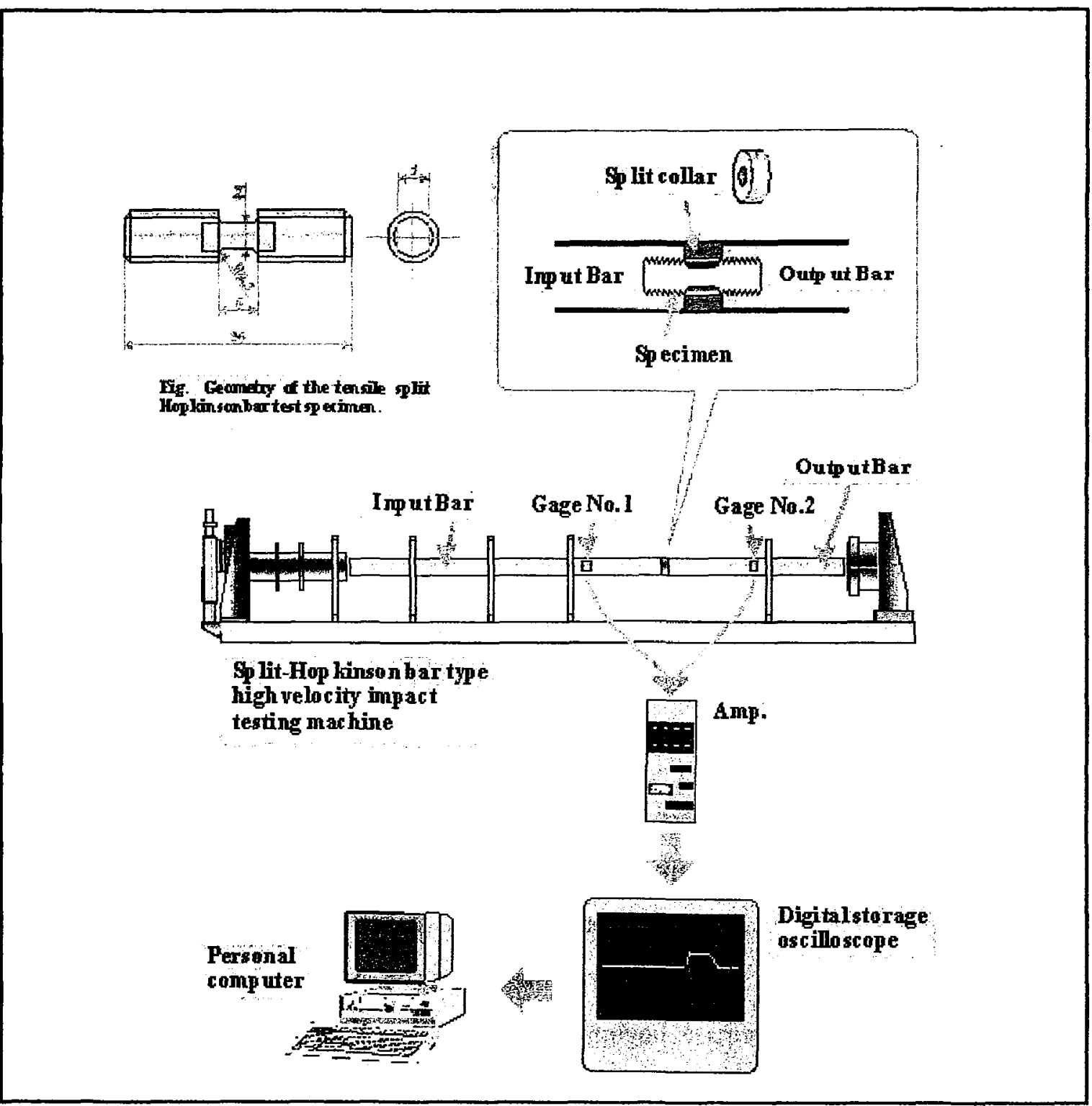

Figure 2.2 - Schematic illustration of the Split Hopkinson Bar Test (SHBT) apparatus [17]. 


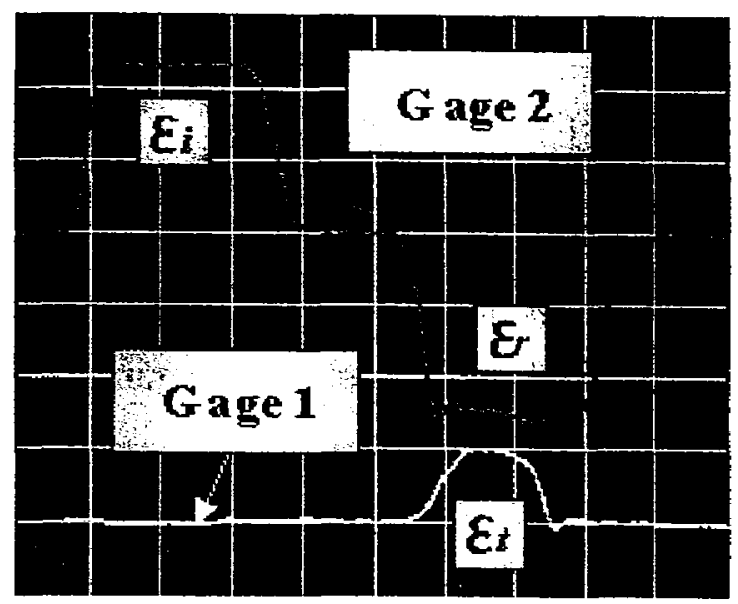

Figure 2.3 - Typical strain measurement history from SHBT [17].

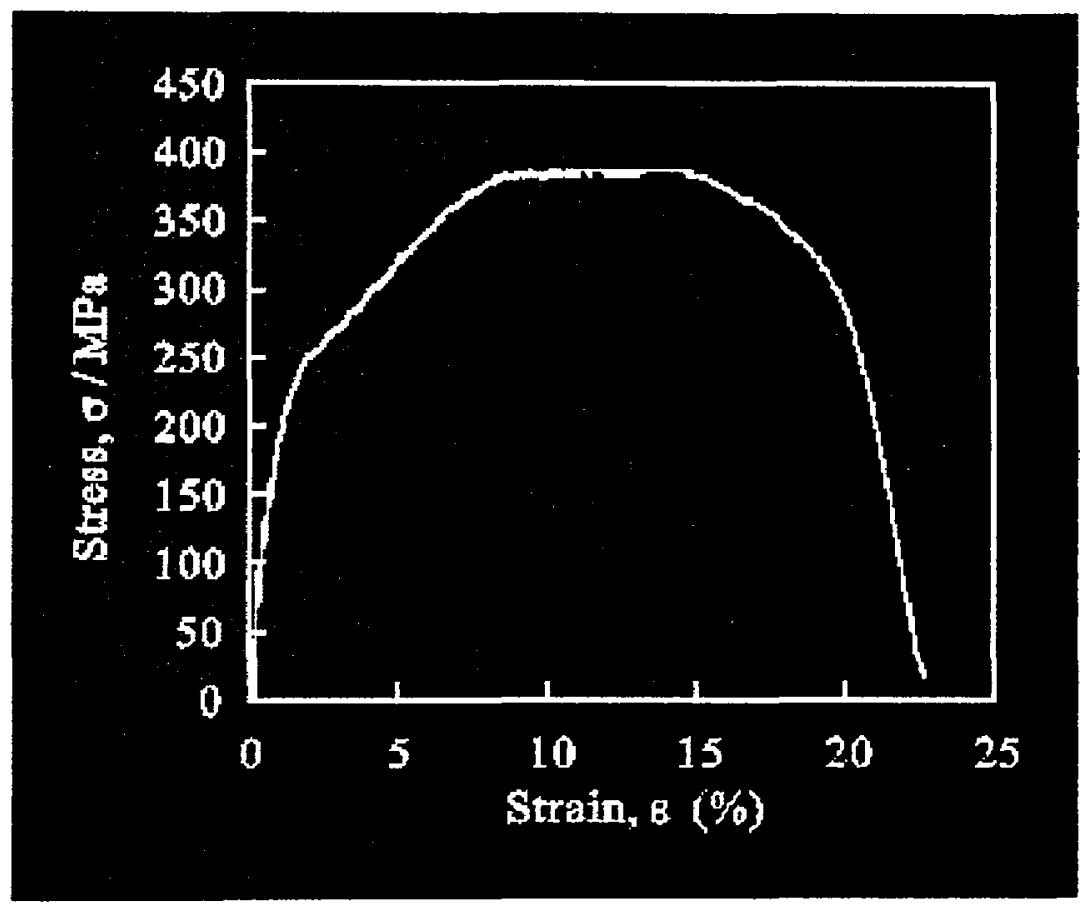

Figure 2.4-Typical stress-strain curve obtained using the SHBT apparatus [17]. 


\subsubsection{STRAIN RATE}

A comparative analysis of the strain rate characteristics that play a prominent role in the dynamic plastic behaviour of both mild steel and aluminium alloys in automotive crash energy absorbing structures shall be provided in this section. There are two schools of thought regarding the application of mild steel and aluminium alloys in automotive crash energy absorbing structures due to the different strain rate sensitivity of both materials. The supporters of mild steel argue that the strain rate sensitivity of mild steel manifests itself as a strengthening effect in structure and thus serves as an additional safety factor. However, the supporters of aluminium argue that the mild steel energy absorption systems could impart unacceptable forces on the human body as the material stiffens under the high strain rates thus limiting the deformation of the system. Aluminium, unlike mild steel, is not sensitive to strain rate and thus shall be more efficient in energy absorption.

\subsubsection{ALUMINIUM ALLOYS}

Data from dynamic uniaxial compression testing conducted by Maiden and Green [18] has demonstrated that the behaviour of aluminium alloys is essentially strain-rate insensitive as shown in Figure 2.5 below. The various strain rates ranging from $0.009 \mathrm{~s}^{-1}$ to $910 \mathrm{~s}^{-1}$ when plotted against the corresponding stress show very little scatter indicating the strain-rate insensitivity of the material. This strain-rate insensitivity may account for the good correlation between rigid, perfectly plastic theoretical methods when compared to corresponding experimental results [18]. 


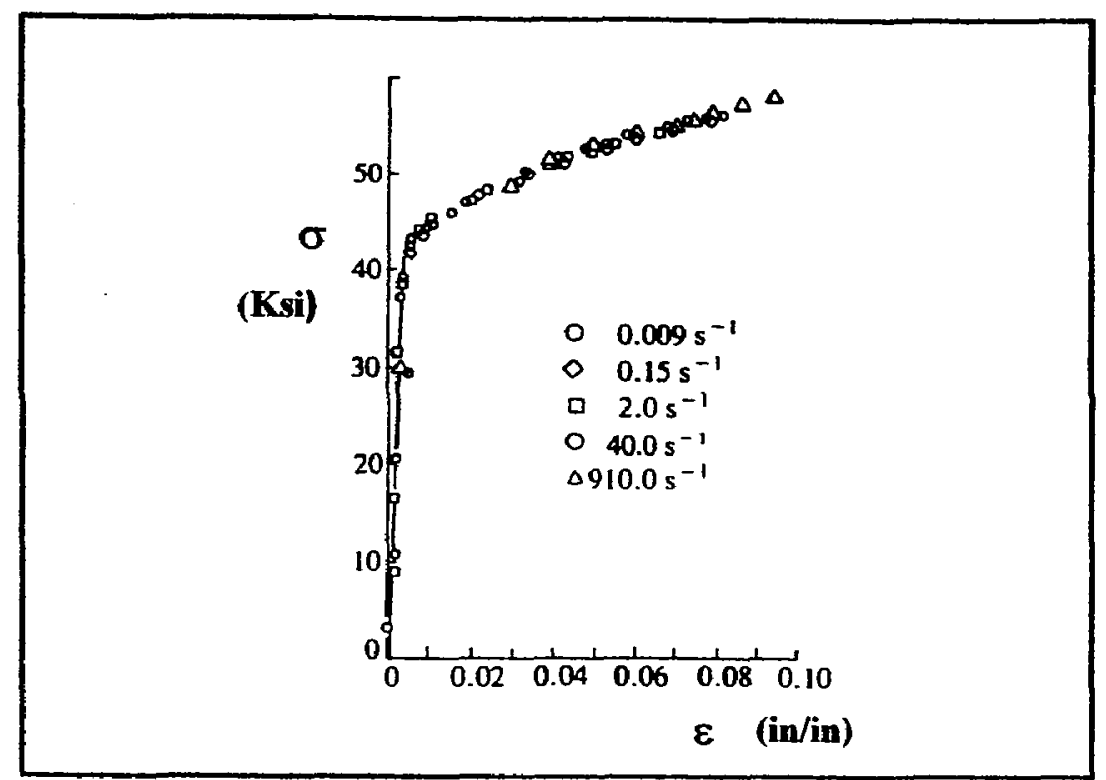

Figure 2.5 - Stress-strain diagram for aluminium alloy generated from dynamic uniaxial compression test at various strain rates [18].

Similarly, Nicholas [15] has demonstrated from dynamic tensile tests that aluminium remains strain-rate insensitive under tensile loading regardless of the stress or strain values used as presented below in Figure 2.6.

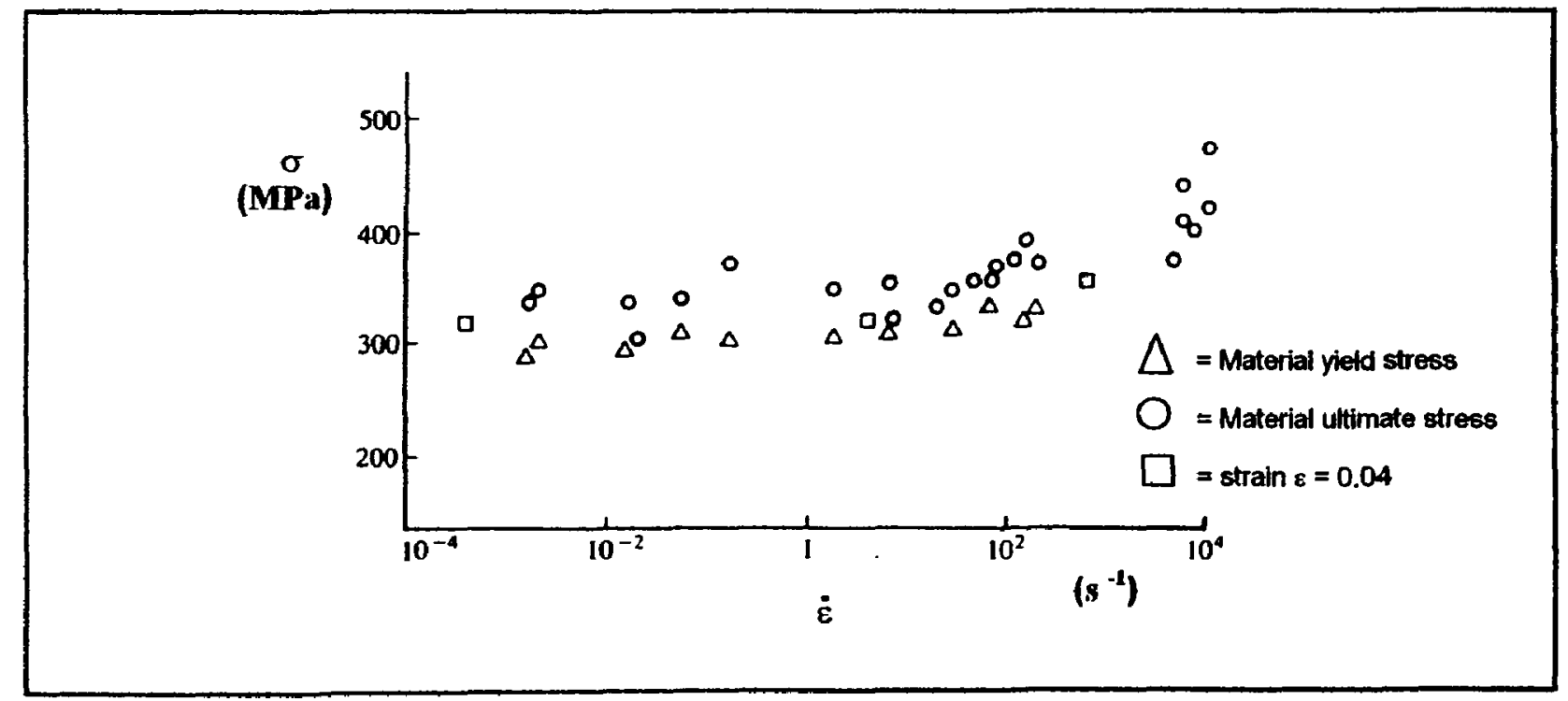

Figure 2.6 - Variation of dynamic uniaxial tensile stresses with strain rate for aluminium alloy [15]. 
Nicholas, Marsh and Campbell $[14,15]$ conducted numerous tests regarding the dynamic shear behaviour of aluminium alloys by using thin-walled tubular specimens subjected to dynamic torsional loads and basically, have found no significant variation in flow stress for increases of shear strain rates up to $10^{3} \mathrm{~s}^{-1}$.

Davies and Magee [10] conducted numerous dynamic bending moment tests on various steel and aluminium alloy sheet metal beams. The strain rates were not measured directly, but could be estimated from the machine cross-head velocities. A dynamic factor was defined as the flow stress at a machine cross-head speed of 5000 inches / minute compared with the flow stress in a static test conducted at 0.1 inches / minute. The dynamic factor for the aluminium alloys tested were found to be very low indicating the strain-rate insensitive behaviour of the material under dynamic bending loads [10]. 


\subsubsection{STEEL ALLOYS}

Due to the extensive applications of mild steel alloys, the material strain characteristics have been widely investigated over the decades and are well understood. Marsh and Campbell conducted extensive dynamic uniaxial impact (compression) tests that indicate a significant increase in the upper yield stress with increase of strain rate and thus revealing the stress-rate sensitivity of steel alloys under dynamic compression type loads. The results of their tests are presented below in Figure 2.7.

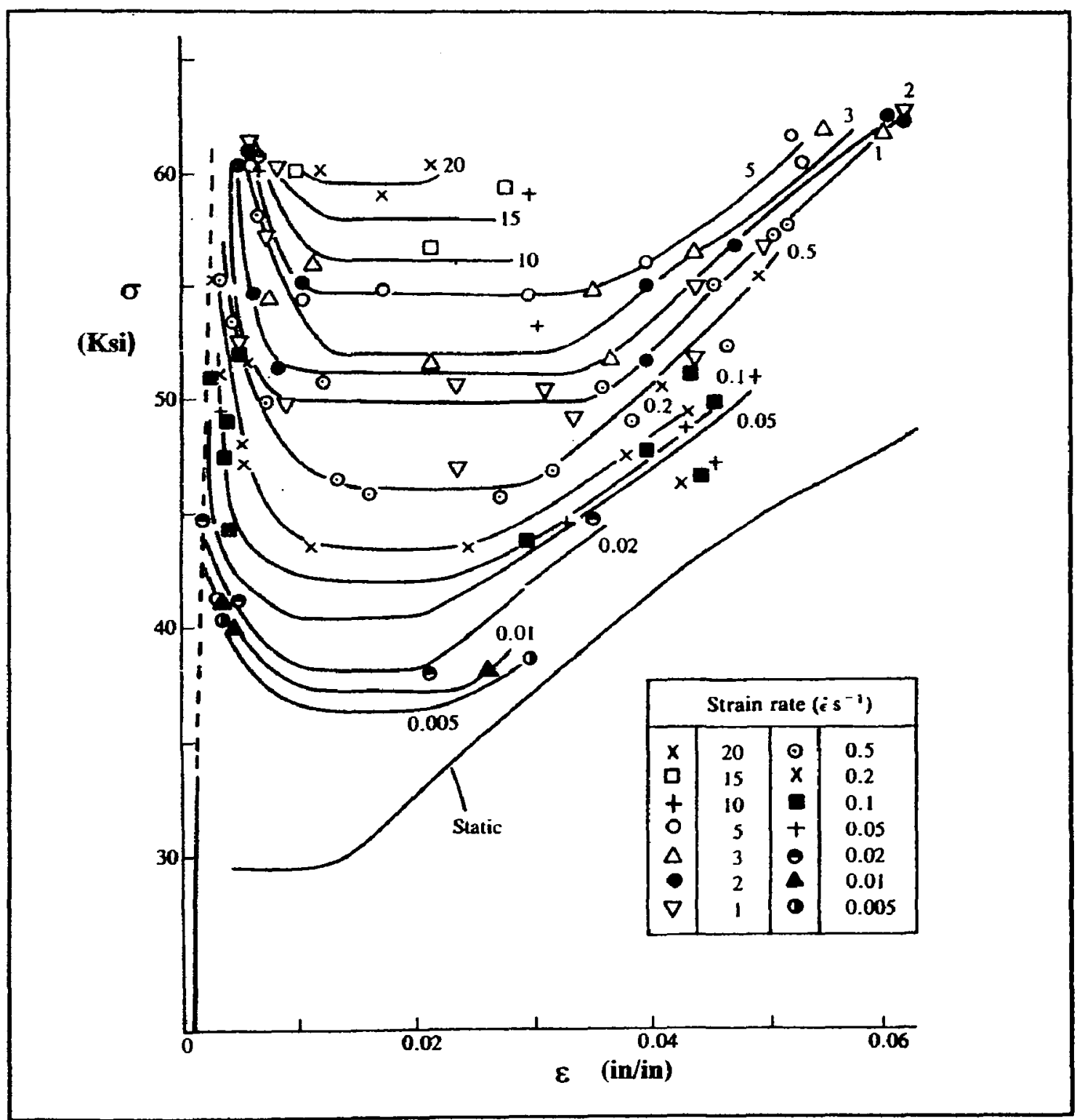

Figure 2.7 - Stress-strain diagram for mild steel alloy generated from dynamic uniaxial compression test at various strain rates [10]. 
With reference to Figure 2.7, at a strain rate of $0.005 \mathrm{~s}^{-1}$ and a strain of $0.02 \mathrm{in}$./in. the corresponding stress is equivalent to approximately $36 \mathrm{Ksi}$, but by increasing the strain rate to $20 \mathrm{~s}^{-1}$ at the same strain level, the corresponding stress increases to $60 \mathrm{Ksi}$. This significant increase in stress is indicative of a material that is strain-rate sensitive.

Similarly, Campbell and Cooper [10] investigated the dynamic tensile behaviour of lowcarbon mild steel specimens up to fracture noticing that the yield stress increases with an increase in strain rate as shown below in Figure 2.8. For the strain rate between $10 \mathrm{~s}^{-1}$ to $100 \mathrm{~s}^{-1}$, the increase in the yield stress has a linear relationship where it increases from $35 \mathrm{Ksi}$ to over $60 \mathrm{Ksi}$ where the strengthening effect is quite significant since it nearly doubles the yield stress of the material.

In addition to the increasing flow stress with increase of strain rate over a wide range of strain rates, Campbell and Cooper [10] noted another interesting material behaviour characteristic from their experimental data. They noticed that the steel alloy fracture strain decreases with increase in strain rate, indicating material embrittlement at higher strain rates.

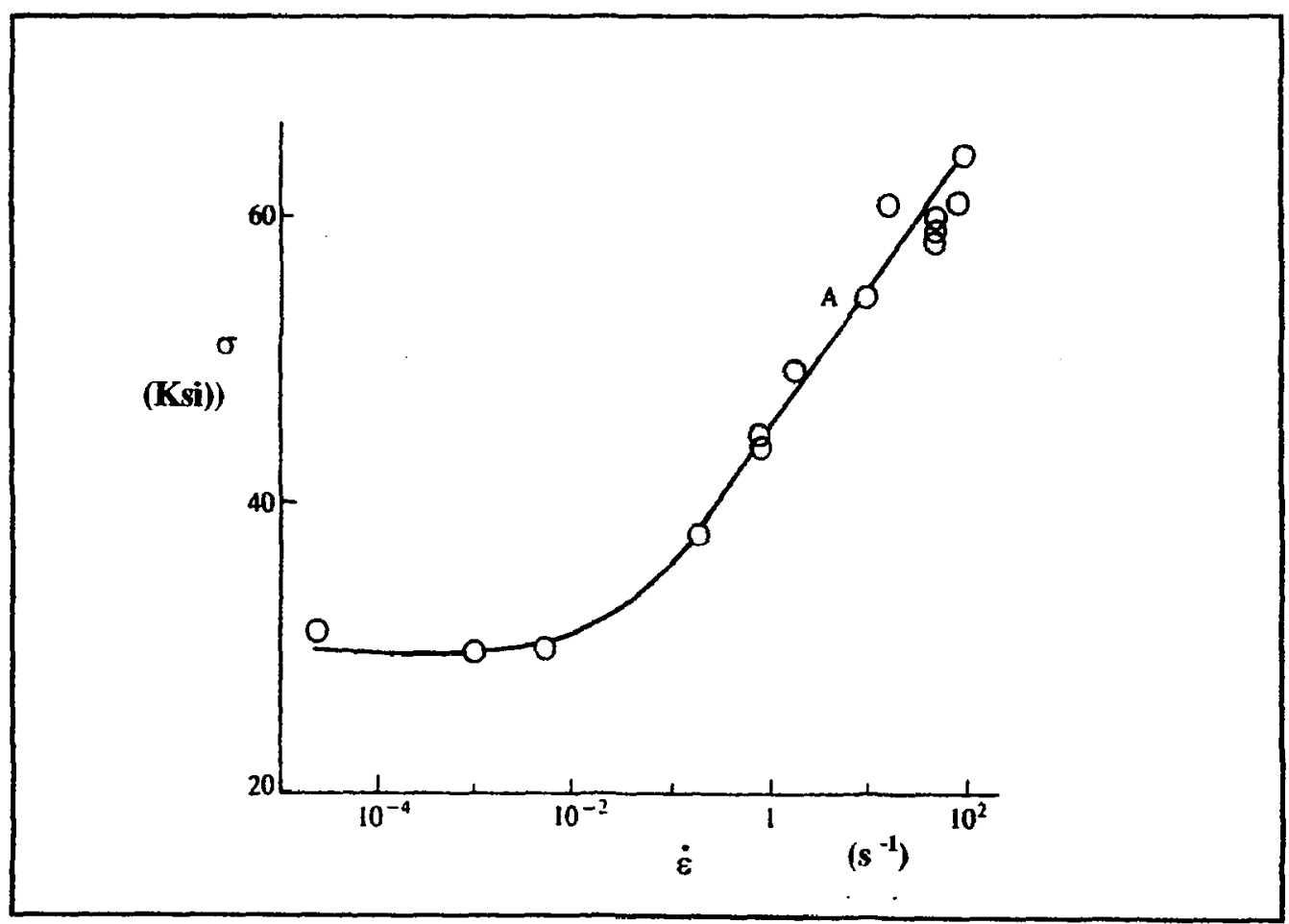

Figure 2.8 - Variation of strength with strain rate for the dynamic uniaxial behaviour of mild steel [10]. 
For the investigation of dynamic bending moment of mild steel beams, Aspden and Campbell constructed an apparatus in which beams were bent with maximum strain rates reaching $20 \mathrm{~s}^{-1}$. The static and dynamic bending moment and their respective angle curves are presented in Figure $2.9[13,19]$.

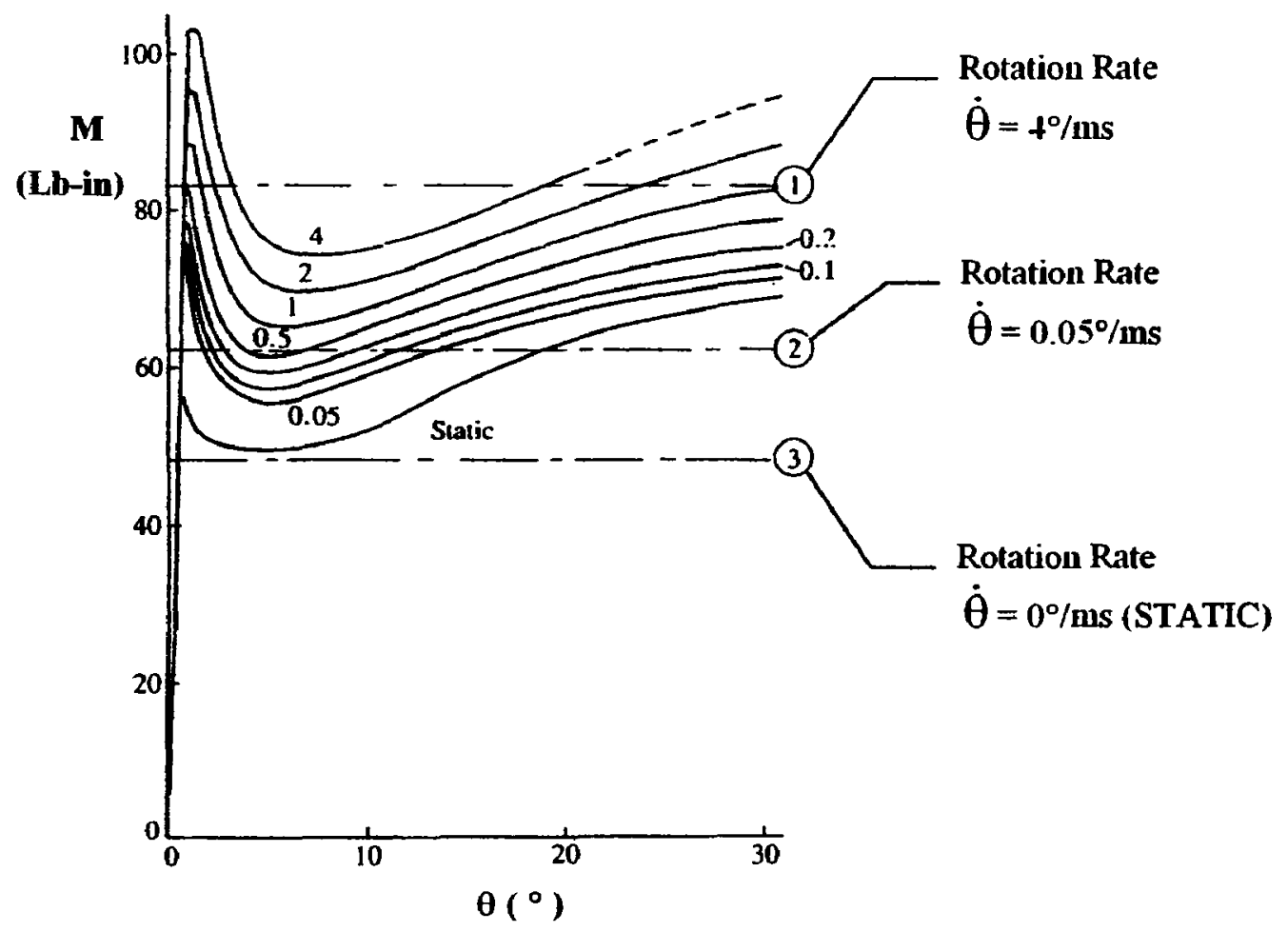

Figure 2.9-Dynamic bending moment-rotation curves for various values of rotation rate $(\dot{\theta})$ for mild steel [10].

Above in Figure 2.9, theoretical predictions using the dynamic bending moment constitutive equation developed based on the Cowper-Symonds constitutive equation where $D=40.4 \mathrm{~s}^{-1}$ and $q=5$ are presented alongside the experimental data for comparison. The horizontal lines numbering 1,2, and 3 in Figure 2.9 represent the calculated theoretical predictions. The numbers on curves are values of $\dot{\theta}$ in degree per milliseconds $(\% / \mathrm{ms})$. 
As can be seen, the predicted theoretical values differ significantly from the experimental results that emphasize the fact that the constitutive equations have limitations in their application, and that experimental derived results are still the primary means of obtaining material behaviour due to strain rate sensitivity. 


\subsection{DYNAMIC PROGRESSIVE BUCKLING}

It has been demonstrated that quasi-static conditions can be assumed for axial impacts with low velocities (up to tens of metres per second) for thin-walled metal tubes since the effects of inertial forces are ignored. Depending on the nature of the impact, neglecting the effects of the inertial forces can be justified in cases where the striking mass $(M)$ is significantly larger than the mass of the tube $(\mathrm{m})$. Although the deformation response of the tube from the impact is in the form of progressive buckling which is a dynamic process, the term "dynamic" becomes more prevalent with strain rate sensitive materials and the high strain rates encountered during the transient wrinkling/lobe forming conditions $[20,21]$.

\subsubsection{PROGRESSIVE BUCKLING MODE}

Wierzbicki and Abramowicz have conducted significant theoretical analyses and research into the crushing behaviour of thin-walled square tubes. They have identified two basic collapse elements that they used to investigate both the static and dynamic progressive buckling of square tubes $[8,21]$.

Unlike circular tube sections, the interaction at the square tube corner region is much more complex and involved and shall be the primary focus of this section. Due to geometric compatibility requirements at the vertical interfaces of the tube elements (corner regions) there are three primary types of progressive buckling modes: Symmetric Type I, Symmetric Type II, and Asymmetric Mixed Mode. The mean crushing force is determined by equating the external work of the axial crushing force to the internal energy required to form either one completer layer of lobes (wrinkles) from four basic collapse elements (sides) or two adjacent layer of lobes with eight basic elements (sides) $[9,10]$. 
Symmetric crushing mode is defined with four of the Type I collapsed sides as shown for each layer of lobes. A b/t ratio greater than $40.8(b / t>40.8)$ is an approximate means to predict symmetric crushing in thin square tubes where $b$ is the length of the tube wall and $t$ is the tube thickness. Another form of symmetric crushing mode involves each layer of lobes in an extensional mode as characterized by Type II and can be predicted to form in thick square tubes with $b / t<7.5[9,10]$.

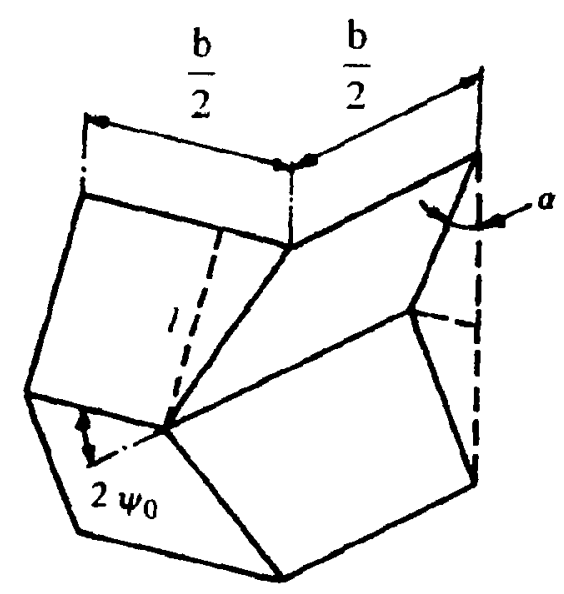

(a)

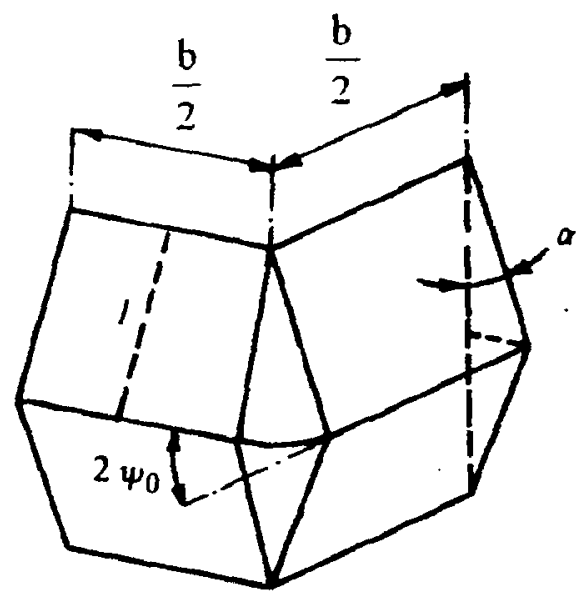

(b)

Figure 2.10-Basic collapse elements: (a) Type I, (b) Type II [10].

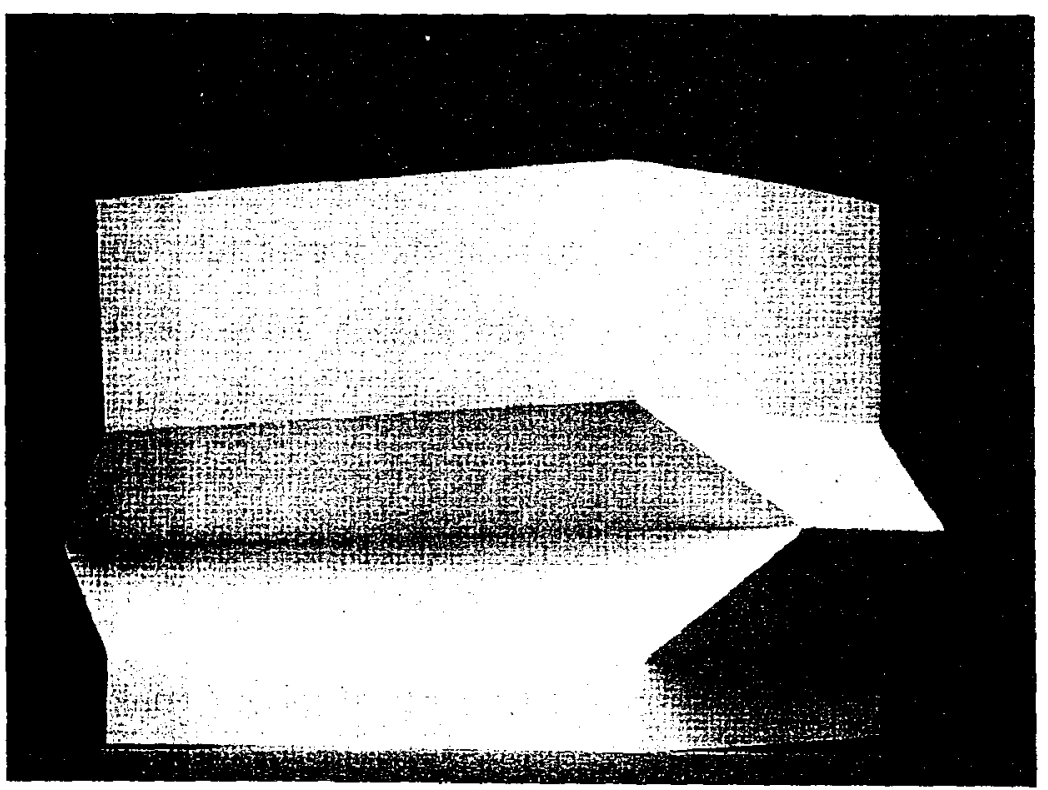

Figure 2.11 - Paper model of square tube symmetric crushing mode Type I [10]. 
The Asymmetric Mixed Mode B progressive buckling is characterized as two adjacent layers of lobes having seven of the sides as Type I collapse and the other as Type II collapse. This type of crushing is predicted to form within the range of $7.5 \leq b / t \leq 40.8$. Experimental data has suggested that the difference between the theoretical crushing forces associated with a Symmetric Mode and an Asymmetric Mode B is small so that either may develop in an actual square tube specimen that has slight imperfections $[9,25]$.

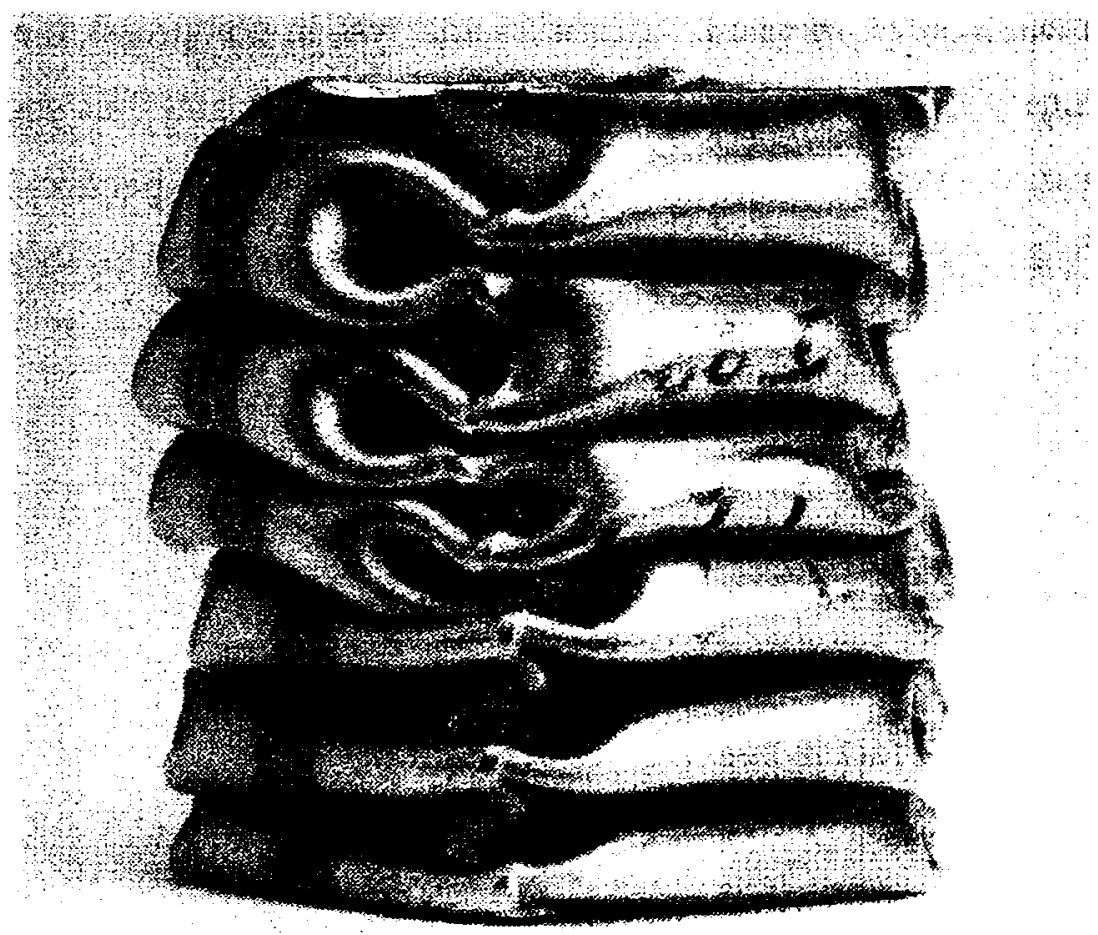

Figure 2.12 - Asymmetric mixed mode B crushing of a thin-walled square tube [1]. 


\subsubsection{SUPER FOLDING ELEMENT METHOD}

Wierzbicki and Abramowicz developed the Super Folding Element Method, which is the predominant analytical solution for evaluating the mean crushing force $\left(\mathbf{P}_{m}\right)$ for the axial progressive crushing of thin-walled columns $[8,9,21]$. The basic folding element proposed consists of three extensional triangular elements and three stationary hinge lines. One contributing flange with width $c$ (which is half of the sectional flange width $b$, thickness $\mathrm{t}$, length $2 \mathrm{H}$ (folding wavelength), and flow stress $\sigma_{0}$. As a result of deformation, three membrane elements are developed near the corner. The three membrane elements consist of one in extension and two in compression along with three horizontal stationary hinge lines with rotation angles of $\theta, 2 \theta$, and $\theta$ as shown. Using the Principal of Virtual Velocity (for further clarification see Appendix I pertaining to Reference [10]), an expression for the equilibrium of the system can be stated as:

$$
\mathbf{P} \cdot \dot{\delta}=\dot{\mathrm{U}}_{\mathrm{b}}+\dot{\mathrm{U}}_{\mathbf{m}}
$$

where:

$P=$ Instantaneous crushing force

$\dot{\delta}=$ Axial displacement rate

$\dot{\mathrm{U}}_{\mathrm{m}}=$ Energy dissipation rate in bending deformation

$\dot{\mathrm{U}}_{\mathrm{b}}=$ Energy dissipation rate in membrane deformation

In Figure 2.13 on the next page, view (a) depicts the geometry before deformation, where as, view (b) is an isometric view after deformation. View (c) is a side view of the folding mechanism where $\delta$ is the net deformation and $\mathbf{P}$ is the applied quasi-static crushing load. 


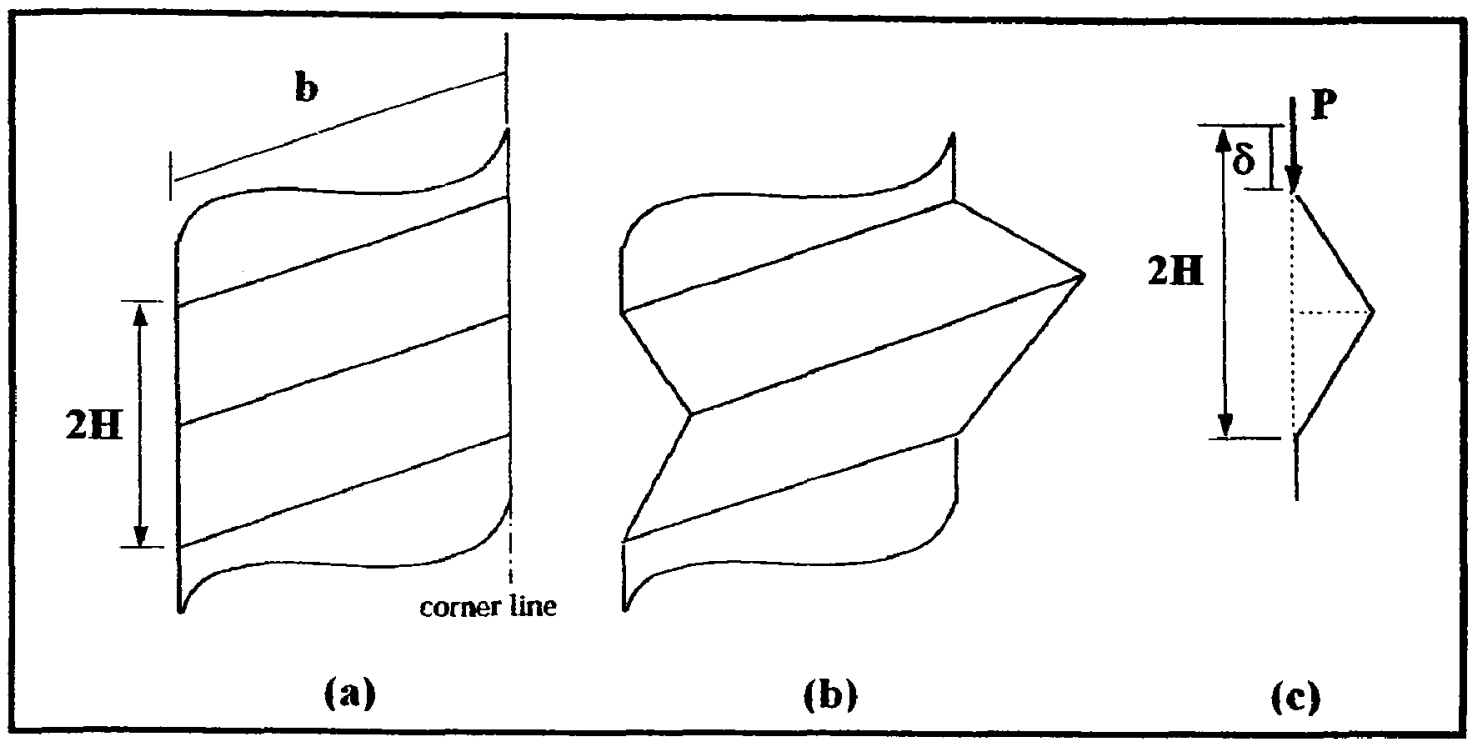

Figure 2.13 - Geometry of basic folding mechanism [8].

Integrating Equation (2.19) for one wavelength in the process of progressive folding, the equilibrium of the system can be further described as:

$$
\mathbf{P}_{\mathbf{m}} \cdot 2 \mathrm{H}=\mathbf{U}_{\mathbf{b}}+\mathbf{U}_{\mathbf{m}}
$$

where $P_{m}$ is the quasi-static mean crushing force.
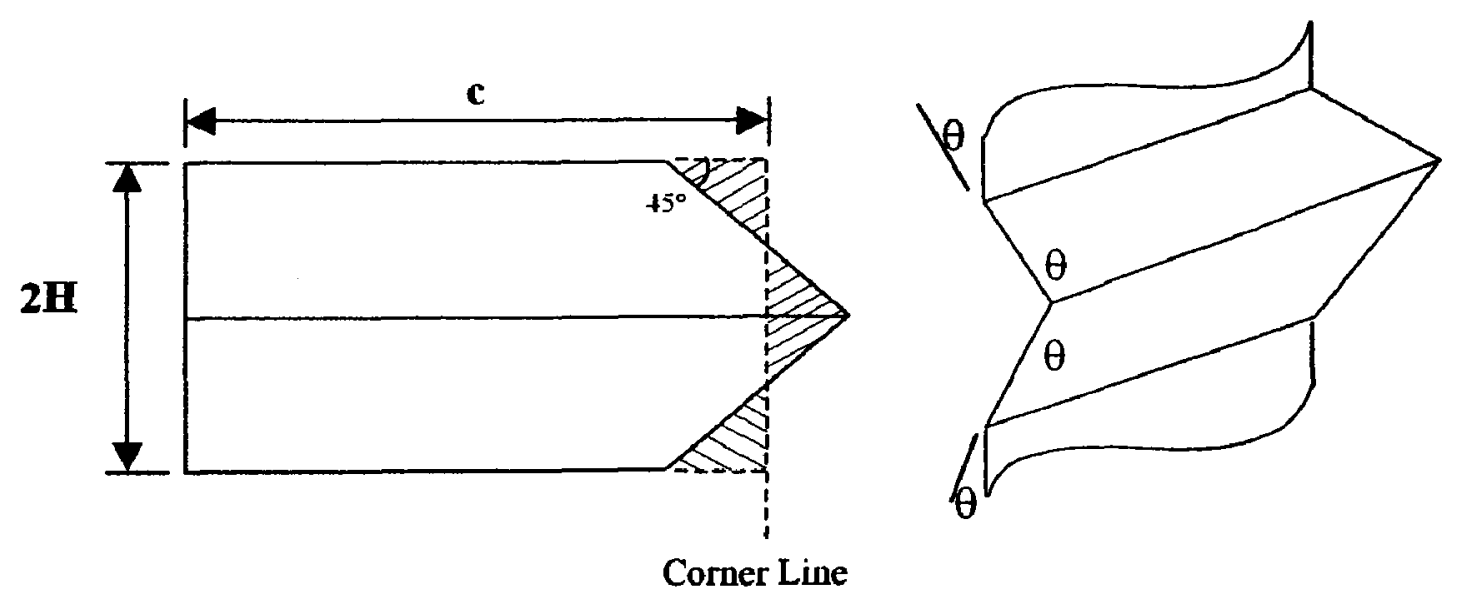

Corner Line

Figure 2.14 - Extensional elements and bending hinge lines [8]. 
Integrating both the extended and compressed area (hatched section in Figure 2.14) provides the membrane energy $U_{m}$ dissipated during the crushing of one wavelength:

$$
U_{m}=\int \sigma_{0} t d s=\frac{1}{2} \sigma_{0} t H^{2}=2 M_{0} \frac{H^{2}}{t}
$$

where $\mathrm{M}_{0}$ is the fully plastic bending moment of the flange idealized as a plate element and is expressed as:

$$
\mathrm{M}_{0}=\frac{1}{4} \sigma_{0} \mathrm{t}^{2}
$$

where $t$ is the flange thickness.

The bending energy $U_{b}$ can be calculated by summing up the energy dissipation at the three stationary hinge lines:

$$
\mathrm{U}_{\mathrm{b}}=\sum_{\mathrm{i}=1}^{3} \mathrm{M}_{0} \theta_{\mathrm{i}} \mathbf{c}
$$

where $\theta_{\mathrm{i}}$ is the rotation angle at each hinge line (recalling each fold consists of three horizontal stationary hinge lines with rotation angles of $\theta, 2 \theta$, and $\theta$ ). To facilitate the analysis, it is assumed that each fold completely flattens the flanges after an axial displacement of $2 \mathrm{H}$ (see Figure 2.15). 


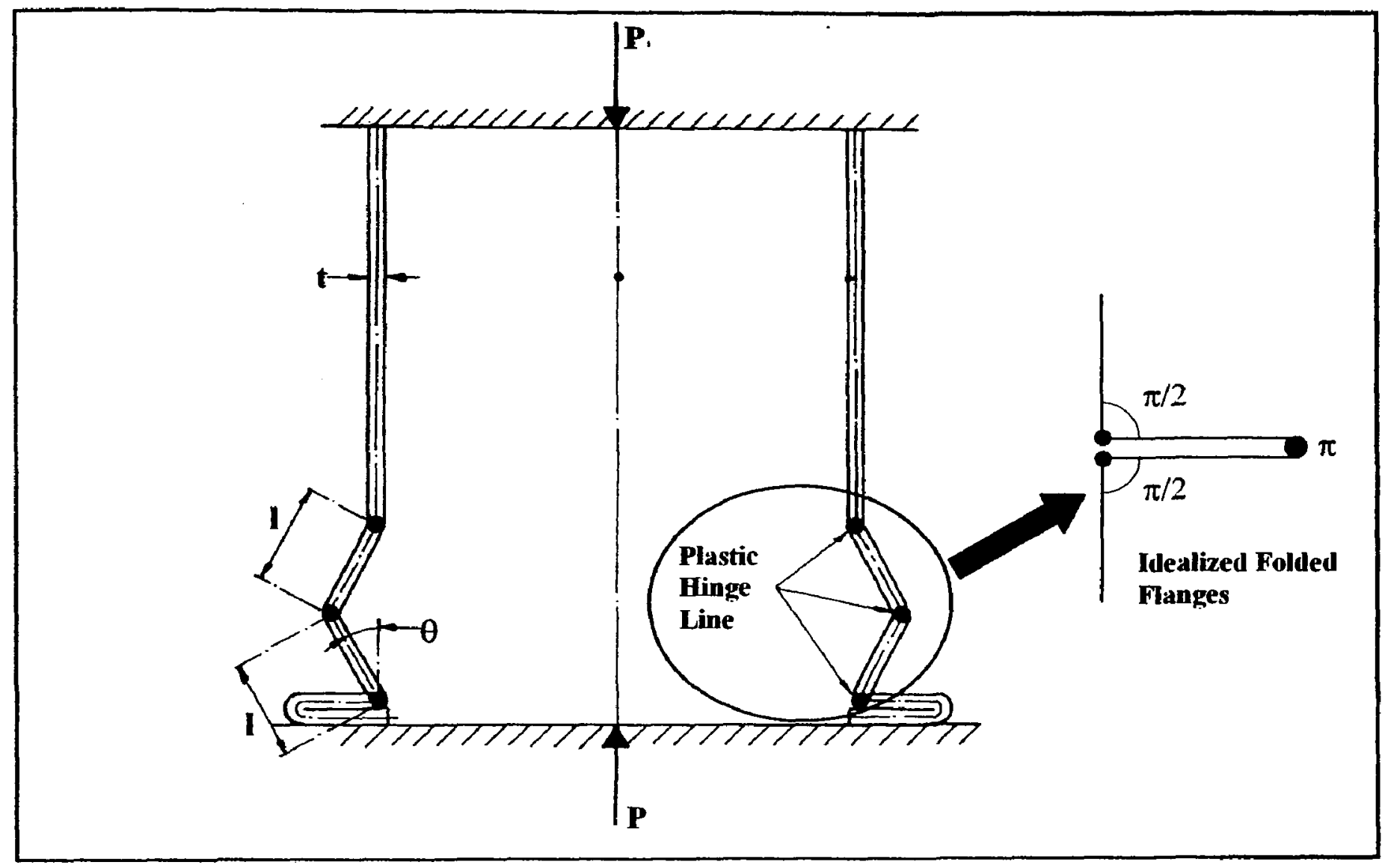

Figure 2.15 - Idealized axial crushing of square tube [10].

Therefore, on the basis of this assumption, the rotation angles at the three hinge lines are $\pi / 2, \pi$, and $\pi / 2$; respectively. Using Equation (2.23) for the defined rotation angles yields the following expression for the bending energy $U_{b}$ :

$$
\mathrm{U}_{\mathrm{b}}=2 \pi \mathrm{M}_{0} \mathrm{c}
$$

Using equations (2.19), (2.21), and (2.24), the mean crushing force $P_{m}$ can be determined by the following expression:

$$
\frac{\mathbf{P}_{\mathbf{m}}}{\mathbf{M}_{0}}=\frac{\mathbf{H}}{\mathbf{t}}+\pi \frac{\mathrm{c}}{\mathbf{H}}
$$


As stated previously, to simplify the analysis, the folds were assumed to be completely horizontal, but in reality this turns out not to be the case since flanges are never completely crushed into a perfectly flat plane. The available crush distance for one wavelength is actually less than $2 \mathrm{H}$, and in fact, Wierzbicki and Abramowicz found that the effective crush distance is typically about $70 \sim 75 \%$ of the wavelength [8,21]. For simplicity, the value is assumed to be 0.75 for the derivation of this analytical solution and thus Equation (2.25) shall be modified accordingly taking the effective crush distance into account:

$$
\frac{\mathrm{P}_{\mathrm{m}}}{\mathrm{M}_{0}}=\frac{4}{3}\left(\frac{\mathrm{H}}{\mathrm{t}}+\pi \frac{\mathrm{c}}{\mathrm{H}}\right)
$$

Equation (2.26) can be further developed to accommodate both multi-flange and multicell sections that are composed of $N$ number of flanges. Assuming the wall thickness is constant over the whole cross-section, the mean crushing force of the section can be calculated by summing up all the contributing flanges:

$$
\frac{P_{m}}{M_{0}}=\frac{4}{3} \sum_{i=1}^{N}\left(\frac{H}{t}+\pi \frac{c}{H}\right)=\frac{4}{3}\left(\frac{N H}{t}+\frac{\pi l}{H}\right)
$$

where $l$ is the total length of the walls (and internal webs) of the cross section. For example, $N=14$ for a double cell section and $N=20$ for a triple cell section.

The folding wavelength $\mathrm{H}$ can be determined by the stationary condition of the mean crushing force:

$$
\frac{\partial P_{m}}{\partial H}=0 \text { which leads to } H=\sqrt{\frac{\pi l t}{N}}
$$


An expression for the mean crushing force for $N$ number of flanges can be expressed as:

$$
P_{m}=\frac{2}{3} \sigma_{0} t \sqrt{\pi N A}
$$

where $\mathrm{A}$ is the material area of the cross section. Thus, the mean crushing force and material area of the cross section for various single, multi-cell/flange profiles that can be used for crash applications are presented in Table 2.2 below:

Table 2.2 - Mean crushing force of various sections $[8,22]$.

\begin{tabular}{c|c|c|}
\hline $\begin{array}{c}\text { Section } \\
\text { Type }\end{array}$ & $\begin{array}{c}\text { Mean Crushing Force } \\
\mathbf{P}_{\mathbf{m}}=\end{array}$ & $\begin{array}{c}\text { Material Section Area } \\
\mathbf{A}=\end{array}$ \\
\hline Square Single Cell & $13.06 \sigma_{0} \mathbf{t}^{53} \mathrm{~b}^{13}$ & $\mathbf{4 b ~ t}$ \\
Double Cell (5 Flanges) & $9.89 \sigma_{0} \mathrm{t}^{32} \mathrm{~b}^{12}$ & $5 \mathrm{~b} \mathrm{t}$ \\
Triple Cell (6 Flanges) & $12.94 \sigma_{0} \mathrm{t}^{32} \mathrm{~b}^{12}$ & $6 \mathrm{~b} \mathrm{t}$ \\
Quadruple Cell & $14.18 \sigma_{0} \mathrm{t}^{32} \mathrm{~b}^{12}$ & $6 \mathrm{~b} \mathrm{t}$ \\
\hline
\end{tabular}

It is important to point out that the theory and numerical solution derived at this stage is based on a rigidly, perfectly plastic material with a constant flow stress $\sigma_{0}$. For a material with considerable strain hardening effects, Wierzbicki and Abramowicz proposed using a modified energy equivalent flow stress such as $[5,8]$ :

$$
\begin{gathered}
\sigma_{0}=\left(\sigma_{0}{ }^{1} \sigma_{0}{ }^{2} \sigma_{0}{ }^{3}\right)^{1 / 3} \\
\sigma_{0}{ }^{i}=\frac{2}{\varepsilon_{0}^{i}} \int_{0}^{\varepsilon_{0}^{i}} \sigma\left(\varepsilon_{u}\right) \varepsilon_{u} d \varepsilon \quad \text { where } \mathrm{i}=1,2,3
\end{gathered}
$$

For the different regions of plastic deformation, Wierzbicki and Abramowicz also proposed using the maximum values of plastic strain $\varepsilon_{u}[5,8]$ :

$$
\varepsilon_{0}^{1}=0.93\left(\frac{t}{b}\right)^{2 / 3} \quad \varepsilon_{0}^{2}=0.69\left(\frac{t}{b}\right)^{1 / 3} \quad \varepsilon_{0}^{3}=1.30\left(\frac{t}{b}\right)^{1 / 3}
$$




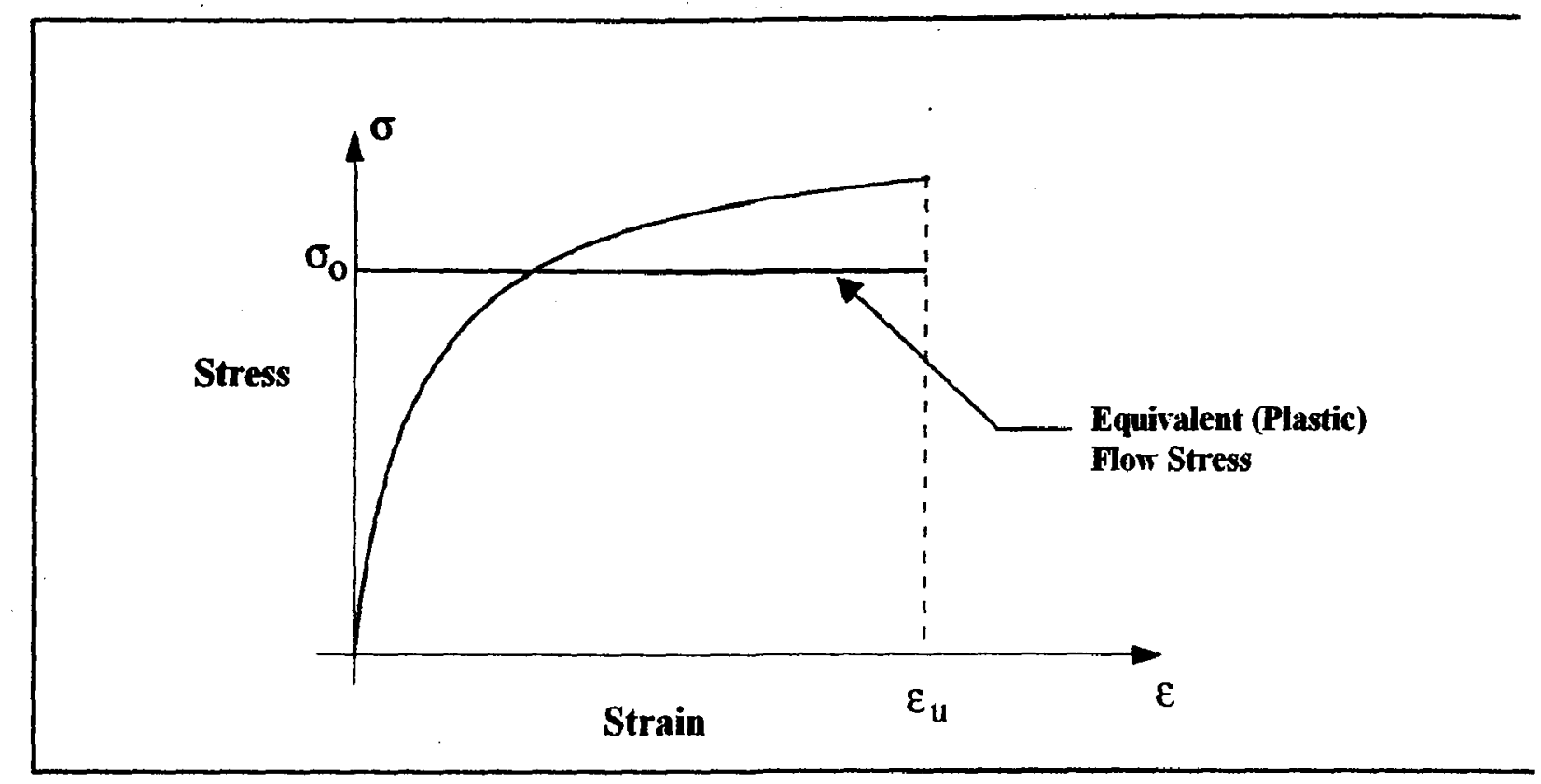

Figure 2.16-Idealized equivalent (plastic) flow stress [9].

An alternate method also proposed by Wierzbicki and Abramowicz that is more expedient for calculating the energy equivalent stress flow and has been shown to have reasonable agreement with experimental data is to take the average value between the yield and ultimate stress $[5,8]$ :

$$
\sigma_{0}=\frac{\sigma_{y}+\sigma_{u}}{2}
$$

where:

$\sigma_{\mathbf{u}}=$ Ultimate stress

$\sigma_{\mathbf{y}}=$ Yield stress

As can be concluded by Figure 2.16, the assumption of the flow stress $\sigma_{0}$ being constant may give reasonable results for a material being rigid, perfectly plastic and insensitive to strain rate. But in materials where significant strain hardening occurs after yielding, this simplification for the stress flow may prove to be a source for inaccurate theoretical values. 


\subsubsection{DYNAMIC AXIAL CRUSHING}

The theory presented in the previous section does not take into consideration the effects of dynamic crushing since the theory was developed for quasi-static conditions where the impact velocities are relatively modest. But most importantly, the material being crushed was idealized as a material being rigid, perfectly plastic and insensitive to strain rate. Further additions to the numerical solution shall be introduced that use the previously presented theory based on the quasi-static response as a foundation to further extend their relevance to more complex material behaviour such as strain rate sensitivity.

\subsubsection{DYNAMIC AMPLIFICATION FACTOR METHOD}

An expression developed by Hanssen introduces a dynamic amplification factor that is based on experimental data obtained through numerous static and dynamic crushing experiments of extruded thin-walled columns [22]. The dynamic amplification factor $D_{\text {amp }}$ is expressed as a function of column width, thickness, mass density, flow stress, and impact velocity:

$$
D_{\text {amp }}=1+C_{\text {ine }}\left(\frac{(b-t) \rho V_{0}^{2}}{t \sigma_{0}}\right)^{1 / 2}
$$

where:

$\rho=$ Mass density

$\mathrm{V}_{0}=$ Impact velocity

$C_{\text {ine }}=$ Dynamic amplification factor 
The dynamic amplification coefficient $\mathrm{C}_{\mathrm{ine}}$ is a function of relative deformation. In the initial stage of the crushing response, $\mathrm{C}_{\text {ine }}$ is larger due to the inertia effect and therefore, to compensate for this effect and to simplify the calculations, an average value of 0.75 is assumed over the entire displacement range. The dynamic mean crushing force $\left(\mathbf{P}_{m, d y n}\right)$ is calculated as:

$$
P_{m, d y n}=D_{a m p} P_{m}
$$

where $P_{m}$ can be obtained referring to Table 2.2 depending on the section characteristics or calculated directly using Equation (2.29). 


\subsubsection{STRAIN RATE SENSITIVITY METHOD}

An alternate method determined by Abramowicz and Jones directly compensates for the strain rate sensitivity of the material. Therefore, Equation (2.29) is modified to compensate for the strain rate sensitivity of the material and the expression for the mean crushing force for a closed section composed of $N$ number of flanges is $[4,9]$ :

$$
\mathrm{P}_{\mathrm{m}, \mathrm{dyn}, \mathrm{st}}=\frac{2}{3} \sigma_{0} \mathrm{t} \sqrt{\pi \mathrm{NA}}\left[1+\left(\frac{\dot{\varepsilon}}{\mathrm{D}}\right)^{\frac{1}{q}}\right]
$$

where:

$\dot{\varepsilon}=$ Plastic strain rate

$\sigma_{0}=$ Static (plastic) flow stress

D = Material constant

$q=$ Material constant

$A=$ Material area of the cross section

$\mathrm{N}=$ Number of flanges that make up the cross section

$\mathrm{t}=$ Thickness of cross section flange

Table 2.3 - Material coefficients for various materials [10].

\begin{tabular}{|c|c|c|}
\hline Material & $\mathbf{D}\left(\mathbf{s}^{-1}\right)$ & $\mathbf{q}$ \\
\hline Mild Steel & 40.4 & 5 \\
\hline Aluminium alloy & 6500 & 4 \\
\hline Titanium (Ti 50A) & 120 & 9 \\
\hline Stainless Steel 304 & 100 & 10 \\
\hline
\end{tabular}

Note: The material constants $\mathrm{D}$ and $\mathrm{q}$ are determined from dynamic uniaxial, or pure shear tests on the material. 
Generally, it is difficult to accurately estimate the strain rates responsible for developing complex deformation patterns in thin walled sections, but Abramowicz and Jones derived an estimate for the mean strain rate of the toroidal corner regions:

$$
\dot{\varepsilon}=\frac{0.33 \mathrm{~V}_{0}}{\mathrm{~b}}
$$

where:

$\dot{\varepsilon}=$ Material mean strain rate

$\mathrm{V}_{0}=$ Impact velocity

$b=$ Flange length

Substituting the expression of Equation (2.37) into Equation (2.36), further refines the expression for the mean crushing force for a closed section composed of $N$ number of flanges:

$$
\mathbf{P}_{\mathrm{m}, \mathrm{dyn}, \mathrm{st}}=\frac{2}{3} \sigma_{0} \mathrm{t} \sqrt{\pi N \mathrm{NA}}\left[1+\left(\frac{0.33 \mathrm{~V}_{0}}{\mathrm{bD}}\right)^{\frac{1}{q}}\right]
$$

For a square tube section, equation (2.38) is rewritten as:

$$
P_{m, d y, s t r}=13.06 \sigma_{0} b^{1 / 3} t^{5 / 3}\left[1+\left(\frac{0.33 V_{0}}{b D}\right)^{\frac{1}{q}}\right]
$$

Correlation of the above theoretical predictions using Equation (2.39) with experimental results on steel tubes composed of square sections, reveals that the theoretical predictions tend towards a lower bound of the experimental values for the dynamic crushing forces. The underestimation of the theoretical predictions can be attributed to the difficulty in 
quantifying the mean strain rate in the corner sections and to some extent the limited experimental data for the strain rate sensitive properties of materials with large strains.

For a typical column undergoing axial crushing, the force-displacement relationship can be integrated to obtain the magnitude of the energy absorbed by the column member due to the impact.

The expression for the energy dissipated by the axial crushing of the column can be defined as:

$$
E=\int_{0}^{d} F(\bar{x}) d \bar{x}
$$

where $d$ is the permanent displacement of the crushed column and $F$ is the crush force in the direction of the load. It can be seen that the integral of Equation (2.40) is equivalent to the area under the force-displacement curve over the displacement range (interval) of 0 to $\mathrm{d}$ (see Figure 2.17).

As a result, the absorbed energy divided by the deformation defines the mean (average) crush load and which is an important parameter to evaluate a structural member's capability for absorbing energy. The mean (average) crush load is defined by the following expression:

$$
F_{\text {mean }}=\frac{E}{d}=\frac{1}{d} \int_{0}^{d} F(\bar{x}) d \bar{x}
$$

where $\mathrm{E}$ is the absorbed energy. For impact scenarios where a stationary member is struck by a mass travelling at a given velocity and the impact energy is full dissipated by the stationary member, the mean (average) crush load can be further be defined by the kinetic energy of the impacting mass divided by the deformation of the stationary member: 


$$
F_{\text {mean }}=\frac{K E}{d}=\frac{1 / 2 M V^{2}}{d}
$$

where $\mathrm{M}$ is the mass and $\mathrm{V}$ is the velocity of the impacting mass.

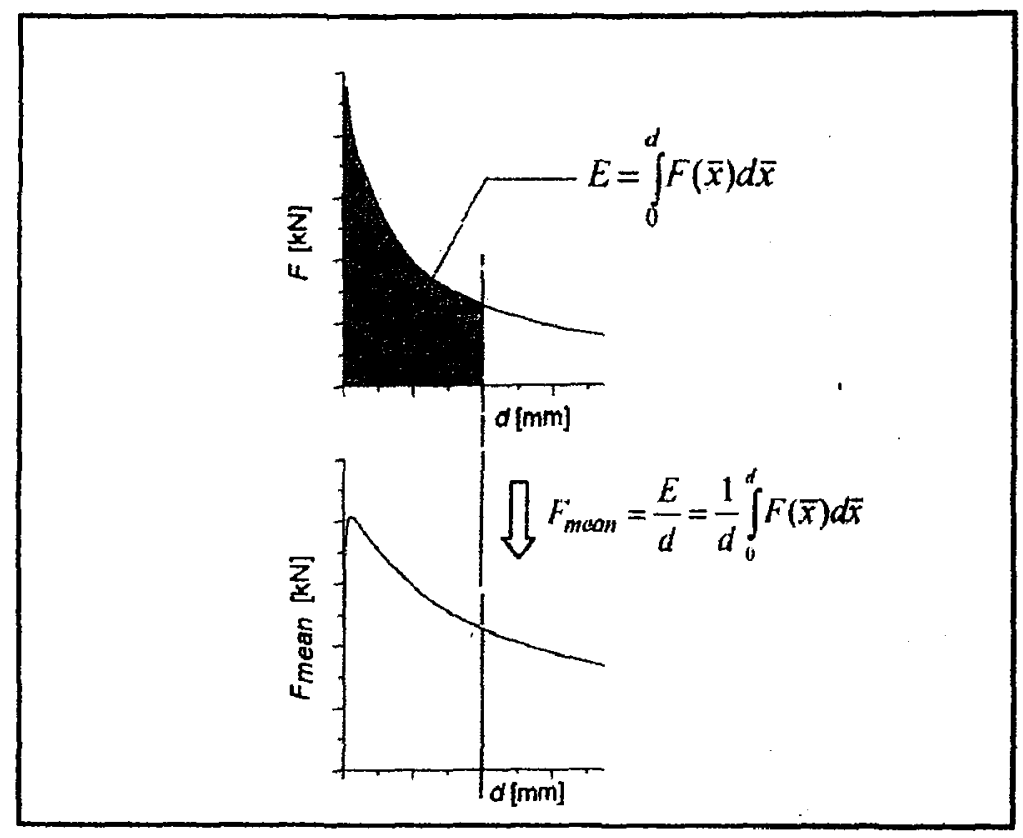

Figure 2.17 - Typical energy $(\mathrm{E})$ and mean crush force $\left(\mathrm{F}_{\text {mean }}\right)$ relationship. 


\subsection{DYNAMIC PLASTIC BUCKLING}

The theory related to dynamic progressive buckling of thin-walled tubular structures subjected to axial impact loading was based on a very important premise. The premise is that the impact loads are applied sufficiently slowly (quasi-static loading) so that neither the axial or lateral inertia effects of the tubular structure play a significant role during the deformation response $[23,24]$. In essence, the duration of the impact loading was much longer than the transient time of the deformation wave that propagates along the length of the tube. Whereas, dynamic plastic buckling is the phenomenon that describes the deformation mechanism of a thin-walled tubular structure that has been subjected to severe dynamic axial loading and significant structural inertia effects. Unlike dynamic progressive buckling with the wrinkling (buckling/lobe formation) confined to one end (normally the impact end), dynamic plastic buckling involves the deformation in the form of wrinkles extending over the entire length of the member. In general, another characteristic of dynamic plastic buckling, mentioned previously, is the influence of inertia forces that tend to favour the development of lateral displacement fields with low mode numbers. This section shall provide an introduction to the dynamic plastic buckling of long cylindrical shell structures.

\subsubsection{DEFORMATION CHARACTERISTICS}

In the case of dynamic progressive buckling, the response of the member as a result of the axial impacting mass was considered one-dimensional because the membrane force $(\mathrm{N})$ and bending moment $(\mathrm{M})$ produced a stress variation through the wall thickness, but did not give consideration to stresses on an orthogonal plane. In the case of dynamic plastic buckling, it is necessary to consider a biaxial stress field due to both axial and radial deformations as shown in Figure 2.18. 


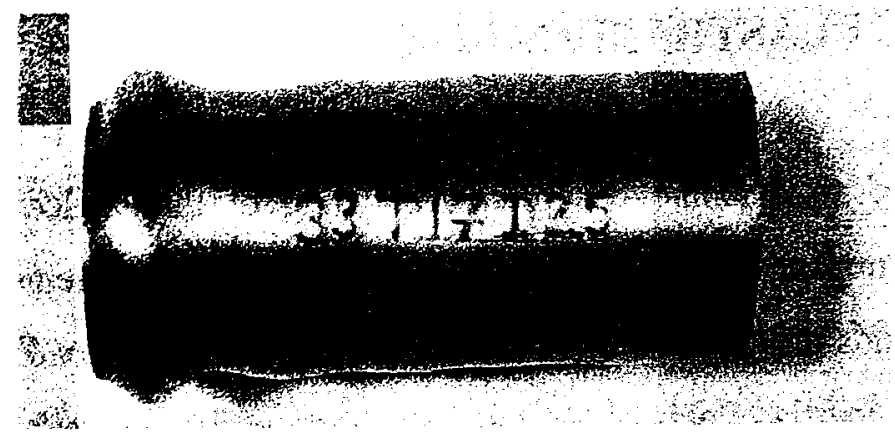

Figure 2.18 - Permanent profile of an aluminium 6061-T6 cylindrical tube that exhibits dynamic plastic buckling deformation [10]

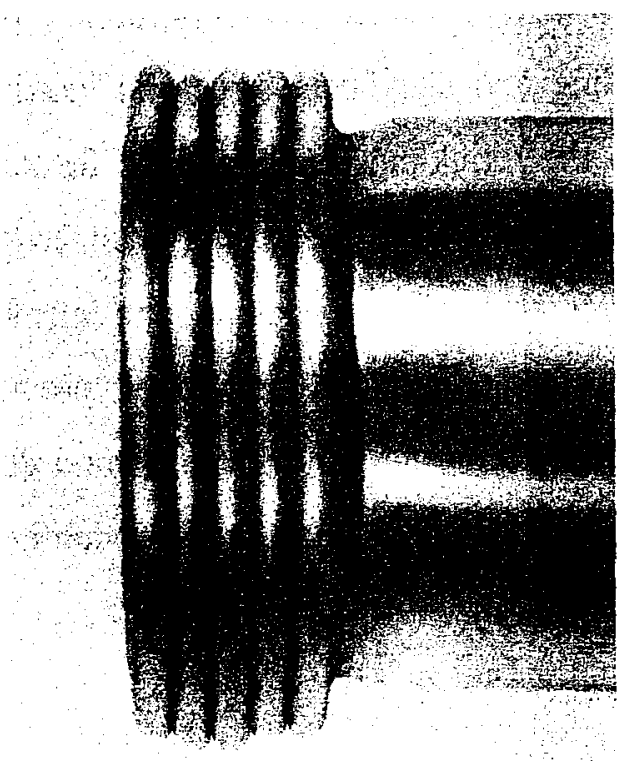

Figure 2.19-Permanent profile of an aluminium 6061-T6 cylindrical tube that exhibits dynamic progressive buckling deformation [10].

Figures 2.18 and 2.19 give valuable insight into the deformation characteristics between the two different buckling forms that contribute to the axial impact energy dissipation of a member: dynamic plastic buckling and dynamic progressive buckling. The extent of axial deformation tends to be less severe for dynamic plastic buckling, with the formation of lobes numbering significantly less compared to the specimen exhibiting dynamic progressive buckling mode of deformation for that of a given impact energy level. 


\subsubsection{GEOMETRIC RELATIONS}

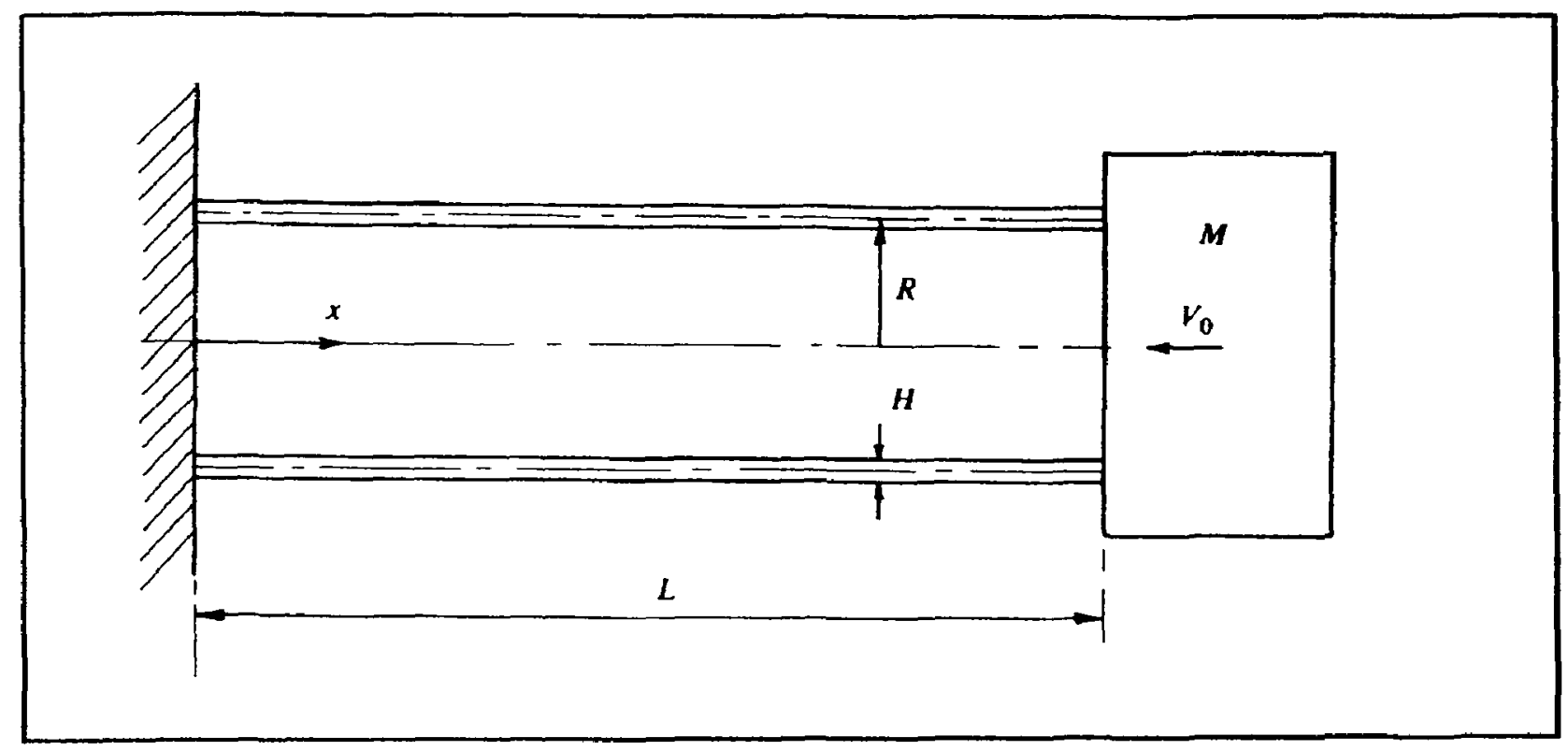

Figure 2.20 - Idealisation of mass $(M)$ with a constant velocity $\left(\mathrm{V}_{0}\right)$ impacting axially on cylindrical shell tube of length $(\mathrm{L})[10]$.

The axisymmetric element of a cylindrical shell as shown in Figure 2.21(c) has an initial length $\mathrm{d} S_{0}$ and a deformed length $\mathrm{d} S$. The initial imperfect transverse (radial) profile is represented by $w^{i}$ and is measured radially inwards from this initial imperfect shape. The axial membrane strain is defined as:

$$
\begin{gathered}
\varepsilon_{\mathrm{x}}=\frac{\mathrm{dS}-\mathrm{dS_{0 }}}{\mathrm{dS_{0 }}} \\
\text { or } \varepsilon_{\mathrm{x}}=\frac{\partial \mathrm{u}}{\partial \mathrm{x}}+\frac{1}{2}\left(\frac{\partial \mathrm{w}}{\partial \mathrm{x}}\right)^{2}+\frac{\partial \mathrm{w}^{i}}{\partial \mathrm{x}} \frac{\partial \mathrm{w}}{\partial \mathrm{x}}
\end{gathered}
$$

e $w$ is the total radial displacement from the initial imperfect profile and $w^{i}$ is the 1 (radial) initial deviation (imperfection) from a perfectly circular profile. 


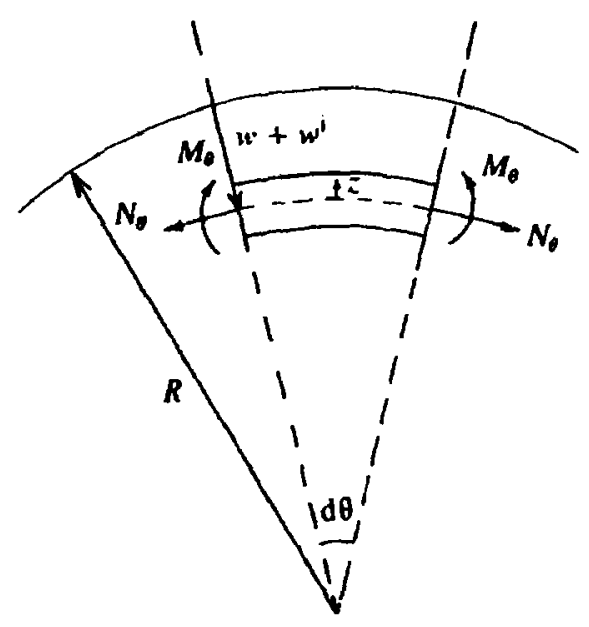

(a)

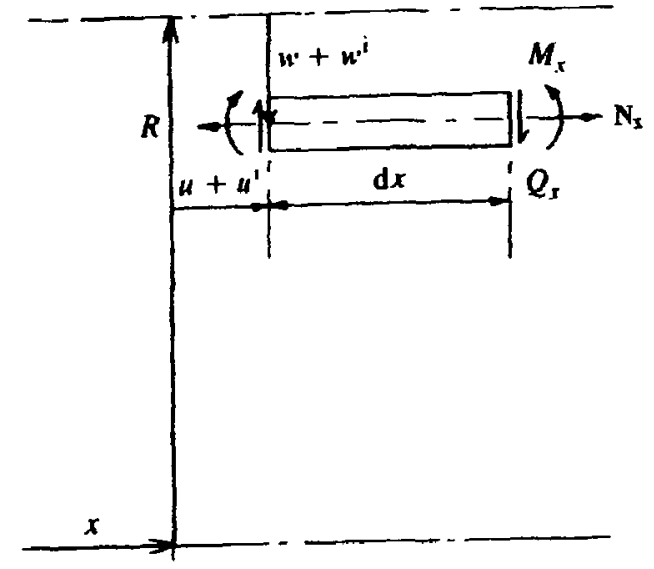

(b)

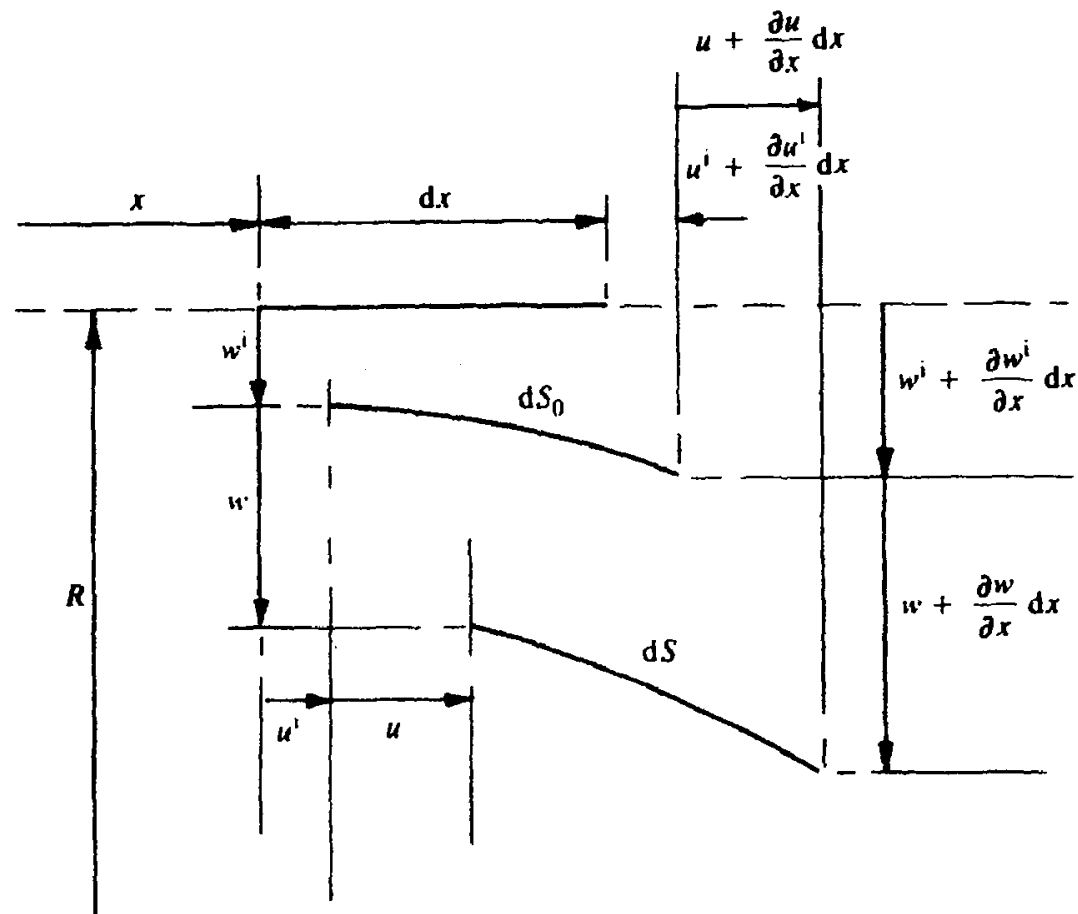

(c)

Figure 2.21-Different perspectives of cylindrical tube shell elements with loading and deformation notation [10]. 
Figures 2.21(a) and 2.21(b) on the previous page, present an axial and circumferential view, respectively, of a cylindrical shell element with the appropriate notation being defined. Similarly, Figure 2.21(c) shows the centre line of the element in the initial ( $\left.\mathrm{dS}_{0}\right)$ and deformed (dS) conditions.

The initial lateral displacement $w^{i}$ is described by the expression:

$$
w^{i}=W_{l}^{i} \sin \left(\frac{\pi x}{L}\right)
$$

where $\mathrm{W}_{1}{ }^{\mathrm{i}}$ is the maximum value of the initial lateral displacement (geometric imperfection), and $\mathrm{L}$ is the length of the tubular member. Neglecting the higher-order terms and assuming $u^{i}=0$, the longitudinal change in curvature can be defined as:

$$
\kappa_{\mathrm{x}}=-\frac{\partial^{2} w}{\partial \mathrm{x}^{2}}
$$

Assuming the deformation of a tube is restricted to remain axisymmetric during the duration of the response, then the circumferential strain can be expressed as:

$$
\varepsilon_{\theta}=\frac{2 \pi\left(R-w^{i}-w+z\right)-2 \pi\left(R-w^{i}+z\right)}{2 \pi\left(R-w^{i}+z\right)}
$$

or approximated as: $\quad \varepsilon_{\theta} \cong-\frac{w}{R}\left(1+\frac{w^{i}}{R}+\frac{z}{R}\right)$

and

$$
\boldsymbol{\kappa}_{\boldsymbol{\theta}}=\mathbf{0}
$$

e $R$ is the mean radius of a tube and $\mathrm{z}$ is measured through the wall thickness from lid-surface as shown in Figure 2.21(a). 


\subsubsection{EQUILIBRIUM EQUATIONS}

The moment, axial and transverse equilibrium equations for a cylindrical shell are presented below, respectively:

$$
\begin{gathered}
\mathrm{Q}_{\mathrm{x}}=\frac{\partial \mathrm{M}_{x}}{\partial \mathrm{x}} \\
\frac{\partial \mathrm{N}_{s}}{\partial \mathrm{x}}-v \frac{\partial^{2} \mathrm{u}}{\partial \mathrm{t}^{2}}=0 \\
\frac{\partial^{2} \mathbf{M}}{\partial \mathbf{x}^{2}}+\frac{\partial}{\partial \mathrm{x}}\left\{\mathrm{N}_{\mathbf{x}}\left(\frac{\partial \mathrm{w}}{\partial \mathrm{x}}+\frac{\partial \mathrm{w}^{\mathrm{i}}}{\partial \mathrm{x}}\right)\right\}+\frac{\mathrm{N}_{\theta}}{\mathrm{R}}\left(1+\frac{\mathbf{w}^{\mathrm{i}}}{\mathrm{R}}\right)-v \frac{\partial^{2} \mathbf{w}}{\partial \mathrm{t}^{2}}=0
\end{gathered}
$$

where:

$v=\rho \times H$

$\rho=$ material density

$\mathrm{H}=$ shell thickness

$t=$ time

Note: Remaining notations not defined are othernise defined in Figure 2.21.

The geometrical relations consisting of Equation (2.44a) and (2.48) along with the equilibrium relations consisting of Equation (2.49) to (2.51) are consistent according to the principle of Virtual Velocities (Reference [10] Appendix $3 \& 4$ ) which can be verified accordingly. 


\subsubsection{CONSTITUTIVE EQUATIONS}

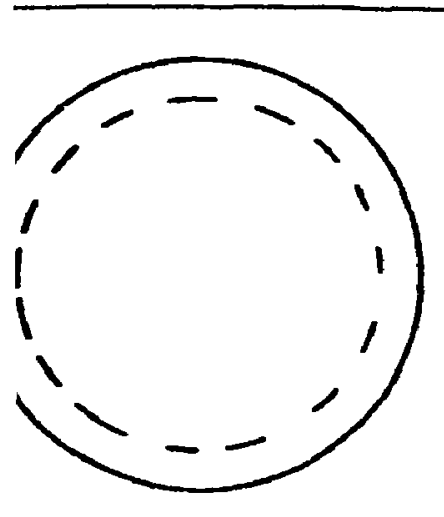

(a)

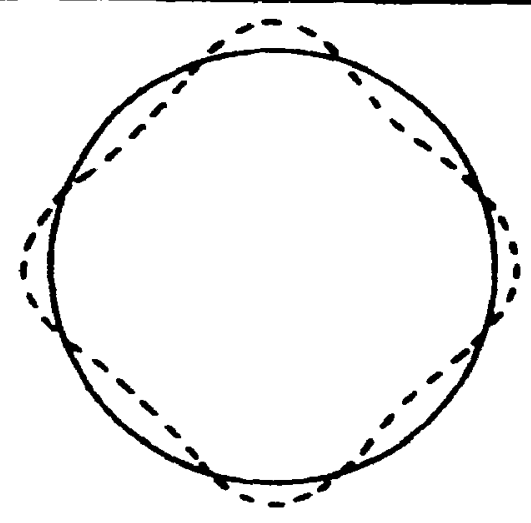

(b)

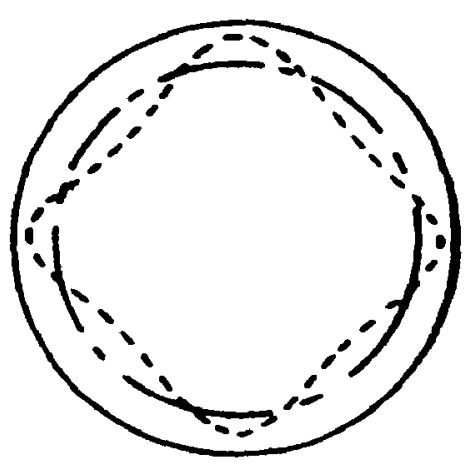

(c)

Figure 2.22 - Idealized response of a cylindrical shell section that is subjected to an axial impact [10].

The section Figure 2.22(a) characterizes the dominant radial displacement ( $\bar{w})$ profile, while section Figure 2.22(b) highlights the perturbation displacement ( $\left.w^{\prime}\right)$ profile. Finally, section Figure 2.22(c) presents the cumulative effect by combining both the dominant radial and perturbation displacement $\left(\bar{w}+w^{\prime}\right)$ profiles. The total radial (or transverse) displacement field may be expressed in the form:

$$
w(x, t)=\bar{w}(t)+w^{\prime}(x, t)
$$

where $\bar{w}(t)$ is the axisymmetric dominant radial displacement and $w^{\prime}(x, t)$ is the perturbed radial displacement. The axial displacement is expressed in a similar fashion in the form:

$$
u(x, t)=\bar{u}(x, t)+u^{\prime}(x, t)
$$

where the $\bar{u}$ and $u$ ' terms are the dominant and perturbed components, respectively. simplification below was introduced by Florence and Goodier after studying the nant behaviour of cylindrical shells [23] :

$$
\frac{\partial \dot{\mathrm{u}}}{\partial \mathrm{x}}=-\frac{\mathrm{V}_{0}}{\mathbf{L}}
$$


where $V_{0}$ is the initial axial impact velocity which is assumed to remain constant during the deformation response, $L$ is the initial length of the tube, and the term in the form of $(\cdot)$ can be rewritten as $\partial() / \partial t$.

Equations (2.44), (2.52), and (2.53) can be used to predict the dominant axial strain $\left(\bar{\varepsilon}_{\mathrm{x}}=\bar{c} \overline{\mathrm{u}} / \partial \mathrm{x}\right)$ using the form of Equation (2.54) which gives:

$$
\dot{\bar{\varepsilon}}_{\mathrm{x}}=-\frac{\mathrm{V}_{0}}{\mathrm{~L}}
$$

Equations (2.47) and (2.52) can be used in a similar fashion to predict the dominant circumferential strain $\left(\widetilde{\varepsilon}_{\theta}=-\bar{W} / R\right)$ which Florence and Goodier described as:

$$
\dot{\bar{\varepsilon}}_{\theta}=\frac{\mathrm{V}_{0}}{2 \mathrm{~L}}
$$

An incompressible material satisfies the expression [10]:

$$
\dot{\bar{\varepsilon}}_{\mathrm{x}}+\dot{\bar{\varepsilon}}_{\mathrm{y}}+\dot{\bar{\varepsilon}}_{\mathrm{z}}=0
$$

and using Equations (2.55) and (2.56) can be used to predict the dominant radial strain rate:

$$
\dot{\bar{\varepsilon}}_{z}=\frac{V_{0}}{2 L}
$$

Therefore, total strain rates based on the work of Florence, Goodier, and Lindberg [24] are given as:

$$
\begin{array}{r}
\dot{\varepsilon}_{x}=-\frac{V_{0}}{L}+z \frac{\partial^{2} \dot{w}^{\prime}}{\partial x^{2}} \\
\dot{\varepsilon}_{\theta}=\left(1-\frac{z}{R}\right)+\left(\frac{V_{0}}{2 L}-\frac{\dot{w}^{\prime}}{R}\right)
\end{array}
$$


For an isotropic plastic material, the Prandtl-Reuss equations can be used to predict both the axial and circumferential stresses. The Prandtl-Reuss constitutive equations for a plastic material assume that an increment in plastic strain (e.g. d $\varepsilon_{\mathbf{x}}$ ) is directly proportional to the corresponding instantaneous deviatoric stress, which is the total stress minus the hydrostatic component [10]. Therefore, the axial and circumferential stresses for a plane stress state $\left(\sigma_{2}=0\right)$ are given as:

$$
\begin{aligned}
& \sigma_{\mathrm{x}}=\frac{2 \sigma_{\mathrm{e}}}{3 \dot{\varepsilon}_{\mathrm{e}}}\left(2 \dot{\varepsilon}_{\mathrm{x}}+\dot{\varepsilon}_{\theta}\right) \\
& \sigma_{\theta}=\frac{2 \sigma_{\mathrm{e}}}{3 \dot{\varepsilon}_{\mathrm{e}}}\left(2 \dot{\varepsilon}_{\theta}+\dot{\varepsilon}_{\mathrm{x}}\right)
\end{aligned}
$$

where the equivalent stress for a rigid plastic material is defined as:

$$
\sigma_{\mathrm{e}}=\sigma_{0}+\mathbf{E}_{\mathbf{h}} \varepsilon_{\mathrm{e}}
$$

where $\sigma_{0}$ is the yield stress and $\mathrm{E}_{\mathrm{h}}$ is the linear strain hardening modulus.

The equivalent strain rate based on the material incompressibility condition is defined as

$$
\dot{\varepsilon}_{\mathrm{e}}=\frac{2}{\sqrt{3}}\left(\dot{\varepsilon}_{\mathrm{x}}^{2}+\dot{\varepsilon}_{\theta}^{2}+\dot{\varepsilon}_{\mathrm{x}} \dot{\varepsilon}_{\theta}\right)^{1 / 2}
$$

Substituting Equations (2.58) and (2.59) into the above Equation (2.63) and neglecting the $\mathrm{z}^{2}$ terms and products of the perturbed quantities gives:

$$
\dot{\varepsilon}_{\mathrm{e}} \cong \frac{V_{0}}{L}\left\{1+\frac{z L}{V_{0}}\left(\frac{4 \dot{w}^{\prime}}{3 R^{2}}-2 \frac{\partial^{2} \dot{w}^{\prime}}{\partial x^{2}}\right)\right\}^{1 / 2}
$$


Equation (2.64) can be written as:

$$
\dot{\varepsilon}_{\mathrm{e}} \cong \frac{\mathrm{V}_{0}}{\mathrm{~L}}\left\{1+\frac{\mathrm{zL}}{2 \mathrm{~V}_{0}}\left(\frac{4 \dot{\mathrm{w}}^{\prime}}{3 \mathrm{R}^{2}}-2 \frac{\partial^{2} \dot{\mathrm{w}}^{\prime}}{\partial \mathrm{x}^{2}}\right)\right\}
$$

Integrating the above Equation (2.65) with respect to time yields the expression for the effective strain:

$$
\varepsilon_{\mathrm{e}} \cong \frac{V_{0}}{L}\left\{t+\frac{z L}{2 V_{0}}\left(\frac{4 w^{\prime}}{3 R^{2}}-2 \frac{\partial^{2} w^{\prime}}{\partial x^{2}}\right)\right\}
$$

More refined expressions for both the axial and circumferential stresses are obtained by substituting the above Equation (2.66) into Equation (2.62) which gives $\sigma_{e}$, which, when further substituted into Equations (2.60) and (2.61) with the other Equations (2.58), (2.59), and (2.63) yields:

$$
\begin{gathered}
\sigma_{\mathrm{x}}=-\sigma^{0}\left(1+\frac{2 \mathrm{~L} \dot{\mathrm{w}}^{\prime}}{3 \mathrm{~V}_{0} R}\right)+\frac{z \sigma^{0} \mathrm{~L}}{3 \mathrm{~V}_{0}}\left(\frac{\partial^{2} \dot{\mathrm{w}}^{\prime}}{\partial \mathrm{x}^{2}}+\frac{4 \dot{\mathrm{w}}^{\prime}}{\mathrm{R}^{2}}-\frac{\mathrm{V}_{0}}{\mathrm{R} \mathrm{L}}\right)+z \mathrm{E}_{\mathrm{h}}\left(\frac{\partial^{2} \mathrm{w}^{\prime}}{\partial \mathrm{x}^{2}}-\frac{2 \mathrm{w}^{\prime}}{3 \mathrm{R}^{2}}\right) \\
\sigma_{\theta}=\frac{4 \mathrm{~L} \sigma^{0} \dot{\mathrm{w}}^{\prime}}{3 \mathrm{~V}_{0} \mathrm{R}}+\frac{2 z \sigma^{0} \mathrm{~L}}{3 \mathrm{~V}_{0}}\left(\frac{\partial^{2} \dot{\mathrm{w}}^{\prime}}{\partial \mathrm{x}^{2}}+\frac{2 \dot{\mathrm{w}}^{\prime}}{\mathrm{R}^{2}}-\frac{\mathrm{V}_{0}}{\mathrm{RL}}\right)
\end{gathered}
$$

where

$$
\sigma^{0}=\sigma_{0}+E_{h} \frac{V_{0} t}{L}
$$

For a perfectly plastic material without any material strain hardening (i.e. $E_{b}=0$ ): $\sigma^{0}=\sigma_{0}$ The axial stress $\sigma_{x}$ acts over an elemental area equivalent to $2 \pi\left(R-w^{i}+z\right) d z$, while the axial membrane force $\mathrm{N}_{\mathrm{x}}$ is defined as a running load with respect to a unit length of the tube circumference. 
The axial membrane force is expressed as:

$$
N_{x}=\int_{-H / 2}^{H / 2} \sigma_{x} \frac{2 \pi\left(R-w^{i}+z\right) d z}{2 \pi\left(R-w^{i}\right)}
$$

If $\frac{w^{i}}{R} \ll 1$, then:

$$
N_{x} \cong \int_{-H / 2}^{H / 2} \sigma_{x}\left(1+\frac{z}{R}\right) d z
$$

Substituting Equation (2.67) into Equation (2.71) and neglecting the $z^{2}$ terms yields the following expression for the axial membrane force:

$$
\mathbf{N}_{\mathrm{x}}=\overline{\mathbf{N}}_{\mathrm{x}}+\mathbf{N}_{\mathrm{x}}
$$

Where $\overline{\mathrm{N}}_{\mathrm{x}}$ is the dominant axial membrane force and is defined as:

$$
\overline{\mathrm{N}}_{\mathrm{x}}=-\sigma^{0} \mathrm{H}
$$

$\mathrm{N}_{\mathrm{x}}$ is the perturbed axial membrane force and is defined as:

$$
N_{x}^{\prime}=-\frac{2 \sigma^{0} H L \dot{w}^{\prime}}{3 V_{0} R}
$$

Similarly, the same can be done for the axial bending moment $\mathrm{M}_{\mathrm{x}}$ as presented below:

$$
\begin{gathered}
M_{x} \equiv-\int_{-H / 2}^{H / 2} \sigma_{x}\left(1+\frac{z}{R}\right) z d z \\
M_{x}=\bar{M}_{x}+M_{x}^{\prime}
\end{gathered}
$$


where:

$$
\overline{\mathbf{M}}_{\mathbf{x}}=\frac{\sigma^{0} \mathrm{H}^{3}}{9 \mathrm{R}}
$$

and

$$
\mathbf{M}_{\mathbf{x}}^{\prime}=-\frac{\mathrm{H}^{3} \sigma^{0} L}{36 \mathrm{~V}_{0}}\left(\frac{\partial^{2} \dot{\mathbf{W}}^{\prime}}{\partial \mathbf{x}^{2}}+\frac{2 \dot{\mathrm{w}}^{\prime}}{\mathbf{R}^{2}}\right)-\frac{\mathbf{H}^{3} \mathrm{E}_{\mathrm{h}}}{36}\left(3 \frac{\partial^{2} \mathbf{w}^{\prime}}{\partial \mathrm{x}^{2}}-\frac{2 \mathbf{w}^{\prime}}{\mathbf{R}^{2}}\right)
$$

The above expression for the perturbed axial moment $\mathbf{M}_{x}$ comprises of two distinct groups of terms. The first group contains the term $\sigma^{0}$ and is known as the directional moment. The last group of terms deals with the material strain hardening modulus $\mathrm{E}_{\mathrm{h}}$ and is known as the hardening moment [10].

In a similar manner, an expression for the circumferential membrane force can be developed from:

$$
N_{\theta}=\int_{-H / 2}^{H / 2} \sigma_{\theta} d z
$$

And also written in the following fashion:

$$
\mathrm{N}_{\theta}=\overline{\mathrm{N}}_{\theta}+\mathrm{N}_{\theta}^{\prime}
$$

Where $\overline{\mathbf{N}}_{\theta}$ is the dominant circumferential membrane force and is considered negligible:

$$
\overline{\mathrm{N}}_{\theta}=0
$$

and $\mathrm{N}_{\theta}{ }_{\theta}$ is the perturbed circumferential membrane force and is defined as:

$$
N_{\theta}^{\prime}=-\frac{4 \sigma^{0} H L \dot{w}^{\prime}}{3 V_{0} R}
$$

The circumferential membrane moment is derived from:

$$
M_{\theta}=-\int_{-H / 2}^{H / 2} \sigma_{\theta} z d z
$$


And also written in the following fashion:

$$
\mathbf{M}_{\theta}=\overline{\mathbf{M}}_{\theta}+\mathbf{M}_{\theta}
$$

Where $\overline{\mathbf{M}}_{\theta}$ is the dominant circumferential membrane moment and is expressed as:

$$
\overline{\mathbf{M}}_{\theta}=\frac{\sigma^{0} \mathrm{H}^{3}}{18 \mathrm{R}}
$$

and $\mathbf{M}^{\prime}{ }_{\theta}$ is the perturbed circumferential membrane moment and is defined as:

$$
M_{\theta}^{\prime}=-\frac{\mathbf{H}^{3} \sigma^{0} L}{18 V_{0}}\left(\frac{\partial^{2} \dot{w}^{\prime}}{\partial x^{2}}+\frac{2 \dot{w}^{\prime}}{R^{2}}\right)
$$




\section{Chapter 3}

\section{NUMERICAL SIMULATION}

The explicit finite element code LS-DYNA was used to simulate the dynamic axial compression of the thin-walled extruded tubes. This fully vectorized, large deformation structural dynamics code has been developed for analyzing transient dynamic response of 3D structures employing as an explicit code the central-difference method in order to solve the equations of motion. It offers many advantages that are critical for efficient and accurate analysis of structural impacts. The research study was conducted in two phases. The first phase consisted of creating and validating finite element models of thin-walled square extruded tubes of varying wall thickness and impact velocities. The results of the numerical simulation were correlated against published experimental data pertaining to similar tube sections to establish the validity of the model. The second phase of the research study used the established model and material parameters that were validated in the first phase to evaluate the crashworthiness behaviour of more complex hexagonal and octagonal thin-walled extruded sections. In addition, the numerical simulation results pertaining to the more complex tube sections were compared against the baseline square section to quantify the improvements in impact energy absorption characteristics. 


\subsection{VALIDATION OF NUMERICAL RESULTS}

The first phase of the research study was deemed critical since it would establish the validity of the material property parameters for the finite element model. The material property parameters would influence the overall plasticity response of the model resulting from the simulated impact. For the validation of the LS-DYNA numerical simulations, it was decided to correlate the numerical simulations with the published experimental results pertaining to Langseth and Hopperstad (Department of Structural Engineering, Norwegian University of Science and Technology) $[5,6,25]$. Both authors have investigated and accumulated extensive experimental data regarding the static and dynamic axial crushing of thin-walled square tube aluminium extrusions. They also established with reasonable accuracy the aluminium alloy AA6060-T4 and T6 material properties of their test specimens using standard tensile testing in conjunction with the work results done by Opheim [26] who found no significant differences in the behaviour of the material in either tension and compression loading. Details regarding the test program and experimental processes are readily available in [5].

To assess the accuracy of the LS-DYNA numerical simulations in predicting the overall post-buckling behaviour of the test specimens, it was decided to select experimental specimens with varying wall thickness and impact velocities. The impact mass for the numerical simulations is kept constant at $55.92 \mathrm{~kg}$ and is in accordance with that used in the tests by Langseth and Hopperstad $[5,6]$.

For the finite element model, all the extruded tubes were modelled using BelytschkoTsay 4-node shell elements with nine integration points through the thickness and one integration point in the element plane with the optimal mesh density for all the models being in the order of $4 \mathrm{~mm} \times 4 \mathrm{~mm}$. The boundary constraints were similar for all the tube configurations with the lower edge fixed (no translation and rotation) and the top end attached to a rigid body (impact plate) member modeled using shell elements. The rigid body member was modelled with one degree of freedom and that being translation along the vertical axis (z-axis) which coincides with the direction of impact. The impact 
velocities were similar to those described in the experimental data $[5,6]$ and were incorporated into the model by defining nodal velocities to the rigid body elements with the simulated total impact mass being constant at $55.92 \mathrm{~kg}$ [5] for all the LS-DYNA model configurations. The length of the extruded tube for all of the model configurations was $310 \mathrm{~mm}$.

For the purposes of the LS-DYNA model validation, a total of 9 experimental specimens fabricated from the aluminium alloy AA6060-T4 were selected and used as a baseline for the LS-DYNA model validation. The same material would also be used for the second phase validation of the more complex sections.

Table 3.1 - Experimental square extrusion tube dynamic test specimens [5].

\begin{tabular}{|c|c|c|c|c|}
\hline Specimen Test & $\begin{array}{c}\mathbf{t} \\
(\mathbf{m m})\end{array}$ & $\begin{array}{c}\mathbf{b} \\
(\mathbf{m m})\end{array}$ & $\begin{array}{c}\mathbf{A} \\
\left(\mathbf{m m}^{\mathbf{2}}\right)\end{array}$ & $\begin{array}{c}\mathbf{V} \\
(\mathbf{m} / \mathbf{s})\end{array}$ \\
\hline EX1.8-1-SQ & 1.80 & 80.0 & 581 & 10.28 \\
EX1.8-2-SQ & 1.80 & 80.0 & 581 & 10.17 \\
EX1.8-3-SQ & 1.80 & 80.0 & 581 & 8.47 \\
EX2.0-4-SQ & 2.00 & 80.0 & 625 & 11.64 \\
EX2.0-5-SQ & 2.00 & 80.0 & 625 & 14.06 \\
EX2.0-6-SQ & 2.00 & 80.0 & 625 & 9.45 \\
EX2.5-7-SQ & 2.50 & 80.0 & 773 & 13.20 \\
EX2.5-8-SQ & 2.50 & 80.0 & 773 & 11.10 \\
EX2.5-9-SQ & 2.50 & 80.0 & 773 & 15.60 \\
\hline
\end{tabular}

where

SQ denotes square section

$\mathrm{V}=$ impact velocity

$\mathrm{b}=$ tube wall width

EX $=$ Experimental

$A=$ section material area

$t=$ section nominal thickness 


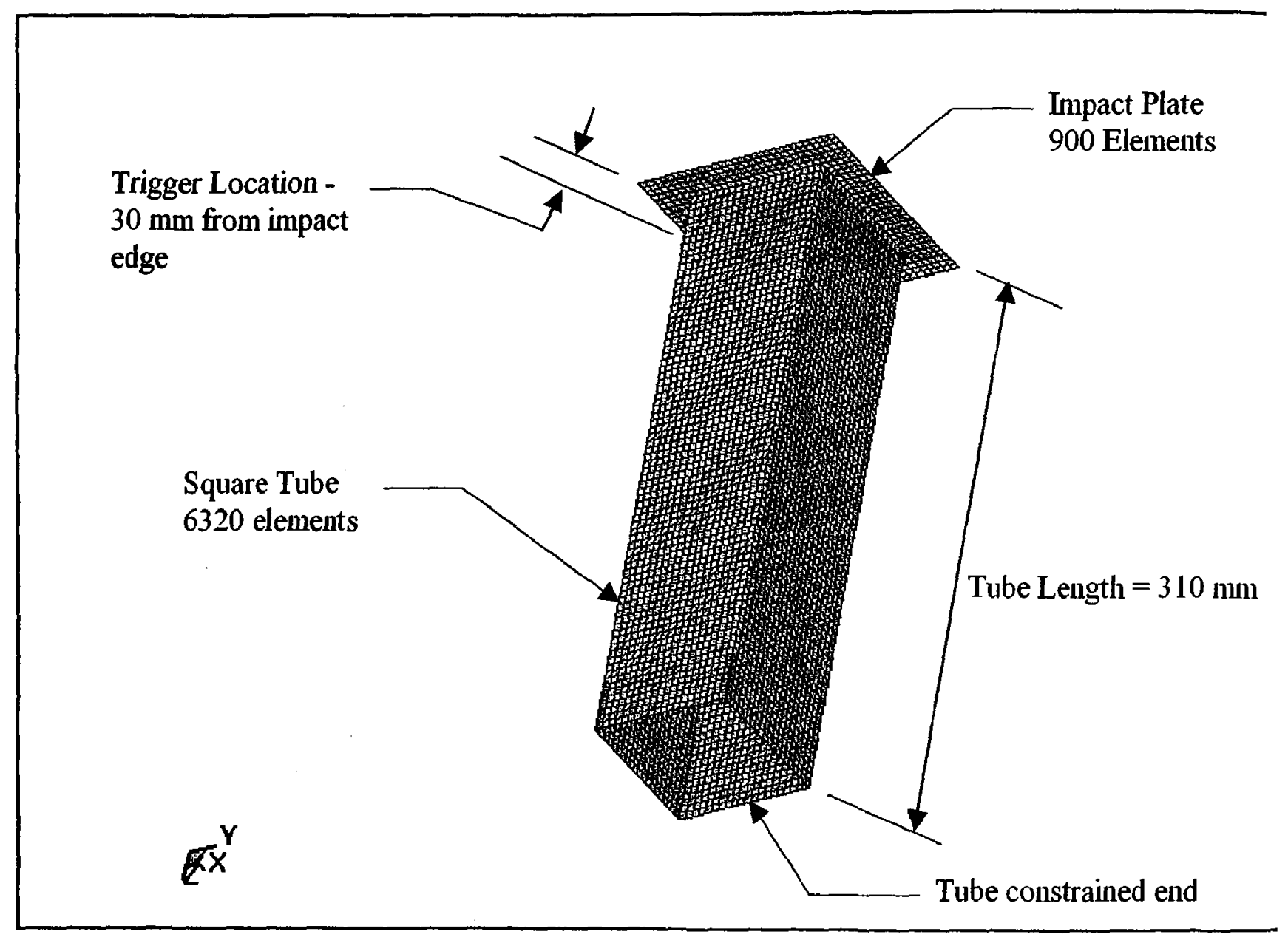

Figure 3.1 - LS-DYNA meshed model depicting the square tube and impact plate specimens. 


\subsection{VALIDATION OF COMPLEX POLYGONAL TUBE SECTIONS}

The second phase of the research extended the validated model parameters and material properties to more complex polygonal tube sections in an effort to evaluate their crashworthiness effectiveness. It was decided to use the computational numerical results from the first phase of the investigation as a baseline to gauge the crashworthiness effectiveness of the more complex shaped members. The common metric established to define the complex shaped hexagonal and octagonal test specimens was the material cross-section area. For each nominal wall thickness (i.e., $1.8 \mathrm{~mm}, 2.0 \mathrm{~mm}$, and $2.5 \mathrm{~mm}$ ) of the square tube section specimen evaluated in the first phase of the investigation, a corresponding tube wall width (b) was determined for each hexagonal and octagonal test specimen to keep the material area constant. Refer to Tables $3.2 \& 3.3$ for more specific geometric details of the tube sections used in the numerical analysis.

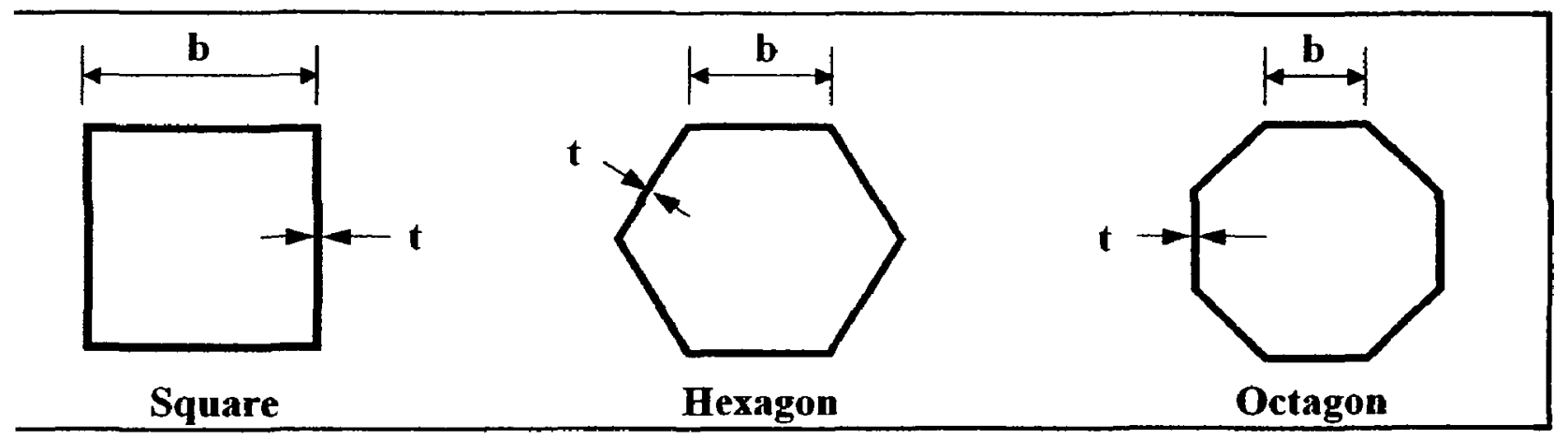

Figure 3.2 - Tube sections used for LS-DYNA numerical simulation analysis.

Table 3.2 - Hexagonal tube section geometric and simulation parameters.

\begin{tabular}{|c|c|c|c|c|}
\hline $\begin{array}{c}\text { LS-DYNA } \\
\text { Model ID }\end{array}$ & $\begin{array}{c}\mathbf{t} \\
(\mathbf{m m})\end{array}$ & $\begin{array}{c}\mathbf{b} \\
(\mathbf{m m})\end{array}$ & $\begin{array}{c}\mathbf{A} \\
\left(\mathbf{m m}^{\mathbf{2}}\right)\end{array}$ & $\begin{array}{c}\mathbf{V} \\
(\mathbf{m} / \mathbf{s})\end{array}$ \\
\hline LS1.8-1-HEX & 1.80 & 53.0 & 581 & 10.28 \\
LS1.8-2-HEX & 1.80 & 53.0 & 581 & 10.17 \\
LS1.8-3-HEX & 1.80 & 53.0 & 581 & 8.47 \\
LS2.0-4-HEX & 2.00 & 53.0 & 625 & 11.64 \\
LS2.0-5-HEX & 2.00 & 53.0 & 625 & 14.06 \\
LS2.0-6-HEX & 2.00 & 53.0 & 625 & 9.45 \\
LS2.5-7-HEX & 2.50 & 53.0 & 773 & 13.20 \\
LS2.5-8-HEX & 2.50 & 53.0 & 773 & 11.10 \\
LS2.5-9-HEX & 2.50 & 53.0 & 773 & 15.60 \\
\hline
\end{tabular}


Table 3.3 - Octagonal tube section geometric and simulation parameters.

\begin{tabular}{|c|c|c|c|c|}
\hline $\begin{array}{c}\text { LS-DYNA } \\
\text { Model ID }\end{array}$ & $\begin{array}{c}\mathbf{t} \\
(\mathbf{m m})\end{array}$ & $\begin{array}{c}\mathbf{b} \\
(\mathbf{m m})\end{array}$ & $\begin{array}{c}\mathbf{A} \\
\left(\mathbf{m m}^{\mathbf{2}}\right)\end{array}$ & $\begin{array}{c}\mathbf{V} \\
(\mathbf{m} / \mathbf{s})\end{array}$ \\
\hline LS1.8-1-OCT & 1.80 & 40.0 & 581 & 10.28 \\
LS1.8-2-OCT & 1.80 & 40.0 & 581 & 10.17 \\
LS1.8-3-OCT & 1.80 & 40.0 & 581 & 8.47 \\
LS2.0-4-OCT & 2.00 & 40.0 & 625 & 11.64 \\
LS2.0-5-OCT & 2.00 & 40.0 & 625 & 14.06 \\
LS2.0-6-OCT & 2.00 & 40.0 & 625 & 9.45 \\
LS2.5-7-OCT & 2.50 & 40.0 & 773 & 13.20 \\
LS2.5-8-OCT & 2.50 & 40.0 & 773 & 11.10 \\
LS2.5-9-OCT & 2.50 & 40.0 & 773 & 15.60 \\
\hline
\end{tabular}

where:

LS denotes LS-DYNA

HEX denotes hexagonal section

OCT denotes octagonal section $\mathrm{b}=$ tube wall width

$A=$ section material area

$\mathrm{V}=$ impact velocity

$\mathrm{t}=$ section nominal thickness

Trigger Location $30 \mathrm{~mm}$ from impact edge

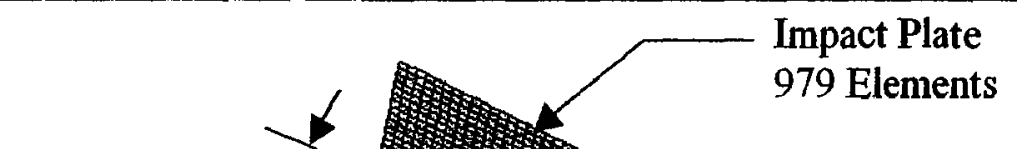

Hexagonal Tube 8010 Elements

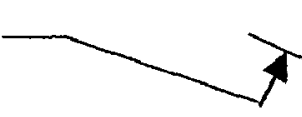
7

Ar

Tube constrained end 


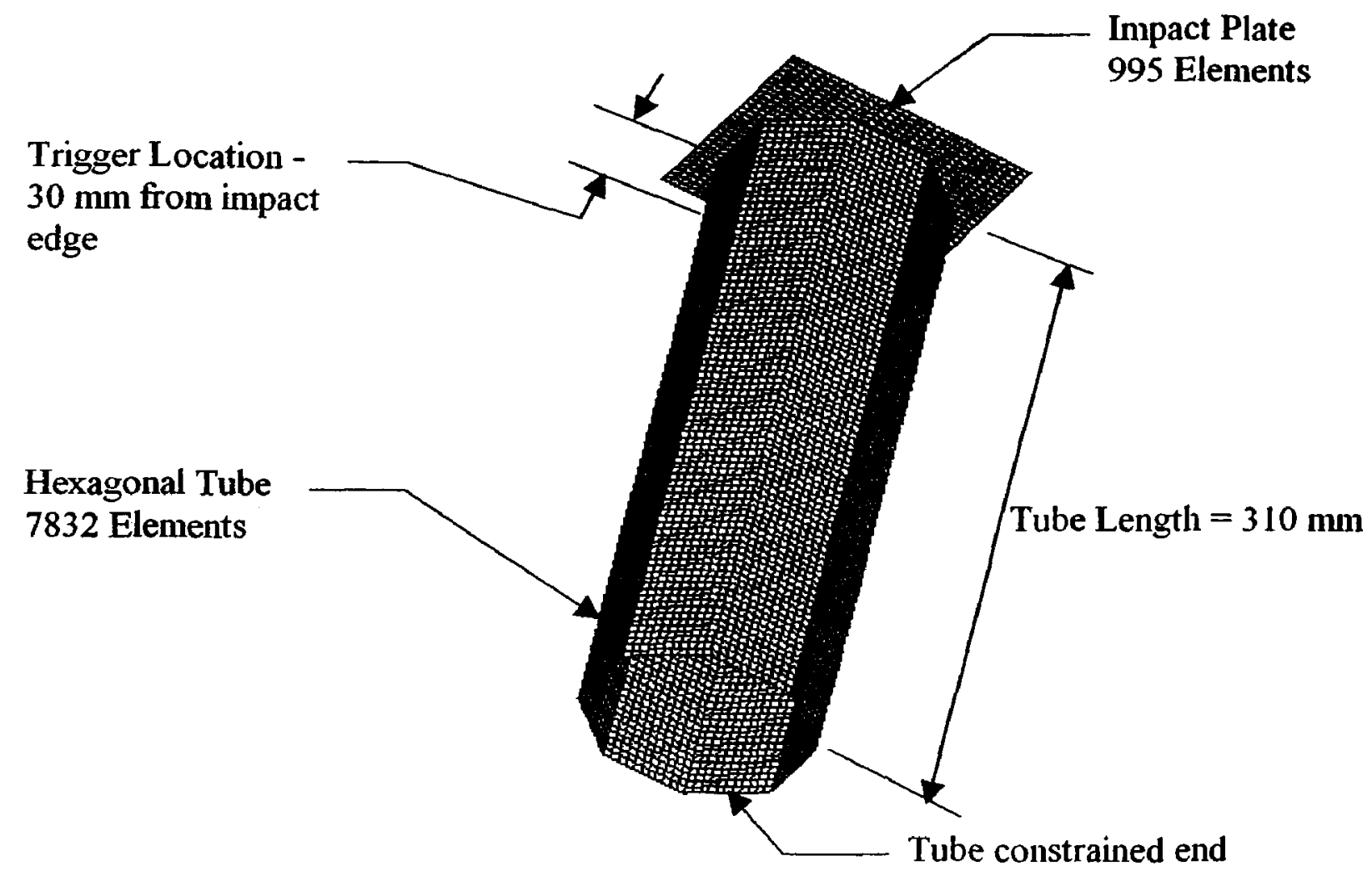

Figure 3.4 - LS-DYNA meshed model depicting the octagonal tube and impact plate specimens.

For the second phase of the research study, the impact mass of $55.92 \mathrm{~kg}$ was kept similar to that used in the first phase along with the specific impact velocities for given sections as defined in Tables 3.1, 3.2, and 3.3. The effort to maintain specific geometric and test parameters consistent between the three varying tube sections would facilitate the comparative analysis and make the results much more relevant in their context.

In all the finite element models, a "trigger" was incorporated in order to induce progressive folding and avoid any global bending mode response that may arise during the post-buckling. It has been shown that for purposes of impact energy dissipation, the essive folding behaviour is more efficient compared to global bending. Automotive ures make extensive use of "triggers" in the front-end members so that stable essive folding is induced in the event of a collision. The "triggers" were placed 30 rom the impact end on all the models, similarly to the experimental models tested by seth and Hopperstad $[5,6]$. 
Figure 3.5 - Trigger profiles utilized for the finite element analysis tube sections.

(a) Square Section

(b) Octagonal Section

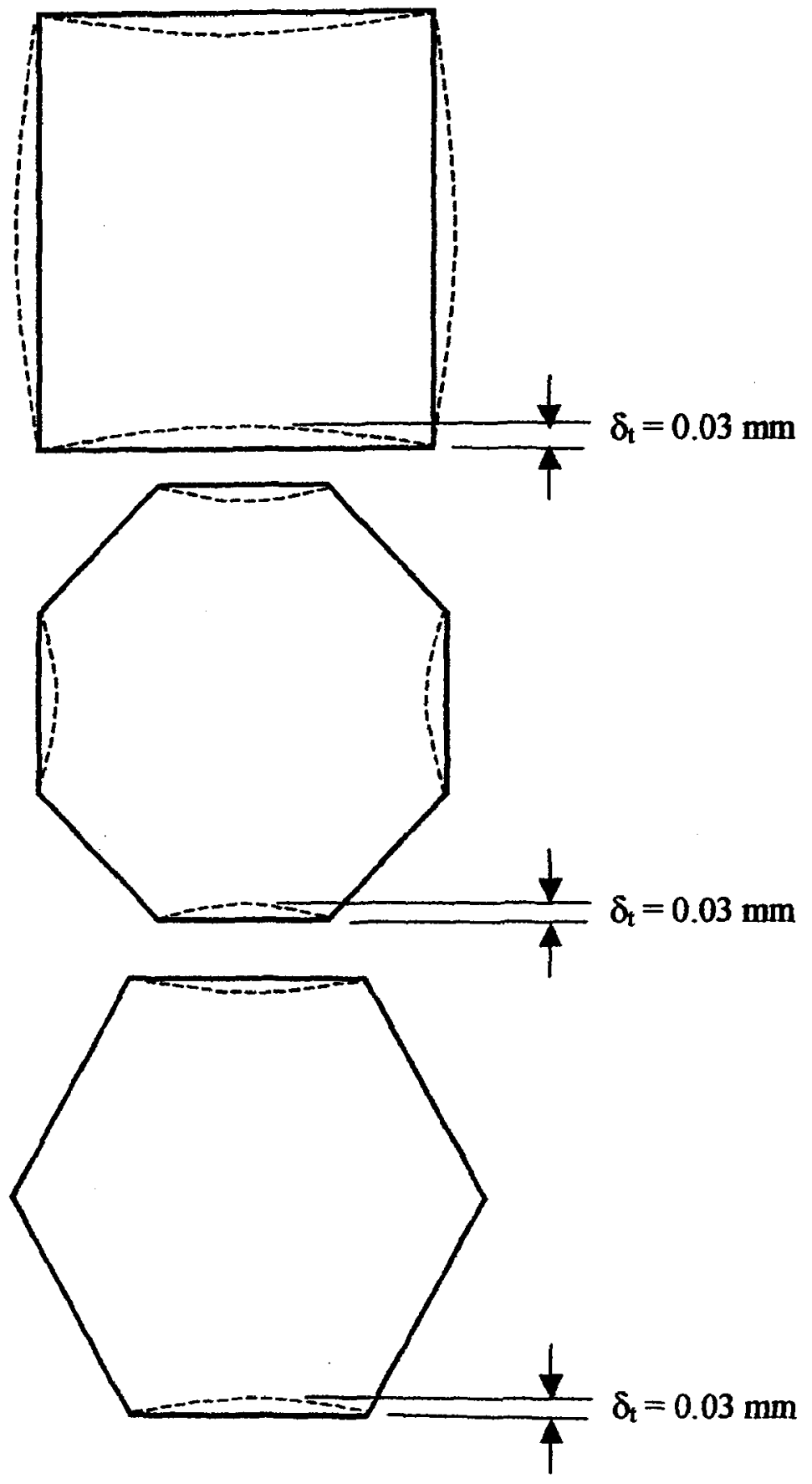

Unfortunately, the use of a "trigger" tends to lower the peak crushing load, but this effect can be minimized by the careful selection of the amplitude $\left(\delta_{t}\right)$ of the "trigger". In spite of this discrepancy, the average force as a function of the axial deformation is not affected significantly and this is the primary parameter that normally dictates the crashworthiness of a component. 
Typically, the peak load manifests itself as very transient in nature and tends to be very localized and short lived in terms of the impact event. The "trigger" amplitude of $\left(\delta_{l}\right)$ of $0.03 \mathrm{~mm}$ was used on all the finite element models and it was consistent with the value used by both Langseth and Hopperstad [5]. The amplitude value of $0.03 \mathrm{~mm}$ is well within the geometrical imperfection (tolerances) of the tube side-walls pertaining to the experimental specimens used by Langseth and Hopperstad. Measurements conducted by Langseth and Hopperstad on the overall wall out-of-flatness for their experimental specimens showed a deviation from nominal with a maximum value of $0.07 \mathrm{~mm}$. Thus the selected amplitude value of $0.03 \mathrm{~mm}$ is well within the measured value of $0.07 \mathrm{~mm}$. 


\subsection{MATERIAL PROPERTY MODEL}

For good correlation due to the non-linear stress-strain curve of aluminium in general, it is quite paramount that the strain hardening properties of the material be included in the material model. It was decided to adopt the Five-Parameter Material Model to develop the true stress-strain curves pertaining to the wall thickness (i.e., 1.8, 2.0, and $2.5 \mathrm{~mm}$ ) of the selected experimental specimens used in the LS-DYNA simulation $[5,25]$.

Formulation for the five parameter material model is given as:

$$
\sigma=\sigma_{0}+\sum_{i=1}^{2} \mathbf{R}_{\mathbf{i}}+\sum_{\mathbf{i}=1}^{2} \mathbf{X}_{\mathbf{i}}
$$

where

$$
\begin{aligned}
& R_{i}=\alpha Q_{i}\left[1-\exp \left(-C_{i} \varepsilon_{p}\right)\right] \\
& X_{i}=(1-\alpha) Q_{i}\left[1-\exp \left(-C_{i} \varepsilon_{p}\right)\right]
\end{aligned}
$$

The parameter $\sigma_{0}$ is the proportionality limit obtained from the uniaxial test, $\varepsilon_{\mathrm{p}}$ is the plastic strain (and is equivalent to $\varepsilon-\sigma / E$ ). The parameter $C_{i}$ governs the rate of change in the isotropic and kinematic hardening variables and $Q_{i}$ represents their asymptotic values. The hardening parameter for this alloy is taken as $\alpha=1.0$ as based on previous studies $[5,25]$.

Any resulting viscous effects were deemed negligible since supporting tests using alloy AA6060 specimens have demonstrated that this particular alloy to be strain rate insensitive [2]. The material constants $\sigma_{0}, C_{i}$, and $Q_{i}(i=1,2)$ were determined by Langseth and Hopperstad based on the true stress-strain curves for each temper and wall thickness and thus were utilized for the developing the true stress-strain curves to be used for the computational analysis material model. The material constants and other relevant parameters obtained via Langseth and Hopperstad via experimental methods are presented in Tables 3.4 and 3.4 [2]. 
The material properties were those of aluminium alloy AA6060-T4. They were based on the uniaxial true stress-strain behaviour of the material fitted to the Five-Parameter Material Model $[5,25]$ and adopted accordingly in the LS-DYNA simulations using the MAT_PIECEWISE_LINEAR_PLASTICITY (Material Type 24) material card. This naterial card allows additional formulations for strain rate effects, but the primary Idvantage is that it allows any arbitrary stress versus strain curve and strain rate dependency For an elasto-plastic material to be defined. The AA6060-T4 stress-strain curves pertaining :o each of the experimental square tube wall thickness (i.e., $1.8 \mathrm{~mm}, 2.0 \mathrm{~mm}$, and $2.5 \mathrm{~mm}$ ) lerived using the Five-Parameter Material Model are presented in Figures 3.6 to 3.8.

Table 3.4 - Material parameters for AA6060-T4 [2].

\begin{tabular}{|c|c|c|c|}
\cline { 2 - 4 } \multicolumn{1}{c|}{} & \multicolumn{3}{c|}{ Nominal Wall Thickness } \\
\hline Parameter & $\begin{array}{c}\mathbf{1 . 8} \\
\mathbf{m m}\end{array}$ & $\begin{array}{c}\mathbf{2 . 0} \\
\mathbf{m m}\end{array}$ & $\begin{array}{c}\mathbf{2 . 5} \\
\mathbf{m m}\end{array}$ \\
\hline$\sigma_{\mathrm{y}}\left(\mathrm{N} \mathrm{mm}^{-2}\right)$ & 56 & 66 & 75 \\
$\mathrm{C}_{\mathbf{l}}$ & 2326 & 399.6 & 25 \\
$\mathrm{Q}_{1}\left(\mathrm{~N} \mathrm{~mm}^{-2}\right)$ & 12.56 & 7.98 & 75 \\
$\mathrm{C}_{\mathbf{2}}$ & 15.82 & 14.54 & 1.9 \\
$\mathrm{Q}_{2}\left(\mathrm{~N} \mathrm{~mm}^{-2}\right)$ & 125.6 & 135.6 & 200 \\
\hline
\end{tabular}

rable 3.5 - Material properties for AA6060-T4 [2].

\begin{tabular}{|c|c|c|c|}
\cline { 2 - 4 } \multicolumn{1}{c|}{} & \multicolumn{3}{c|}{ Nominal Wall Thickness } \\
\hline Parameter & $\begin{array}{c}1.8 \\
\mathbf{m m}\end{array}$ & $\begin{array}{c}\mathbf{2 . 0} \\
\mathbf{m m}\end{array}$ & $\begin{array}{c}2.5 \\
\mathbf{m m}\end{array}$ \\
\hline$\sigma_{0.2}\left(\mathrm{~N} / \mathrm{mm}^{-2}\right)$ & 71 & 76 & 82 \\
$\sigma_{\mathrm{u}}\left(\mathrm{N} / \mathrm{mm}^{-2}\right)$ & 158 & 167 & 171 \\
$\varepsilon_{\mathrm{u}}(\%)$ & 18 & 18 & 19 \\
$\mathrm{E}\left(\mathrm{N} / \mathrm{mm}^{-2}\right)$ & 68200 & 68200 & 68200 \\
$\mathrm{n}$ & 10 & 10 & 10 \\
\hline
\end{tabular}




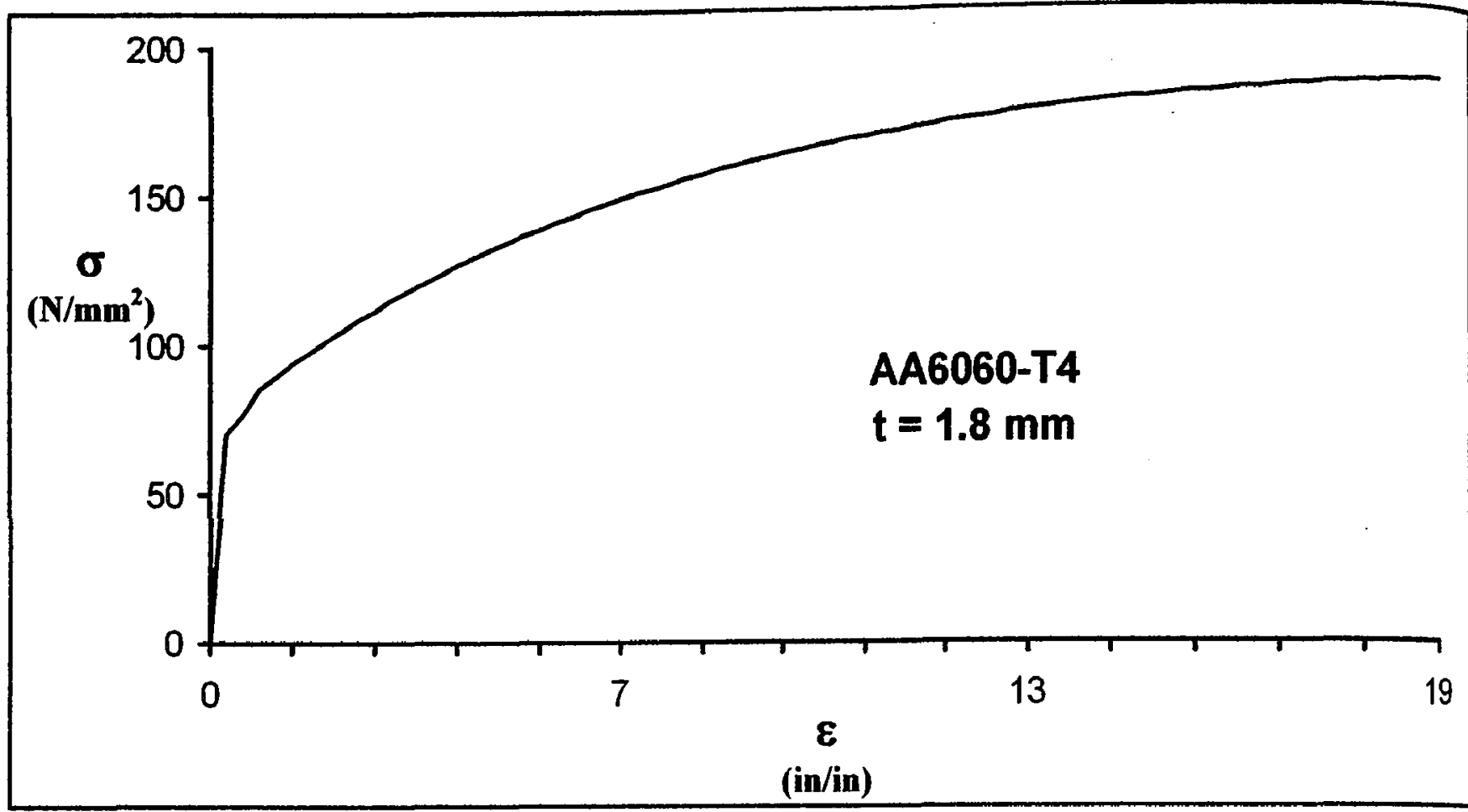

Figure 3.6 - True stress-strain curve of AA6060-T4 alloy $\mathrm{t}=1.8 \mathrm{~mm}$.

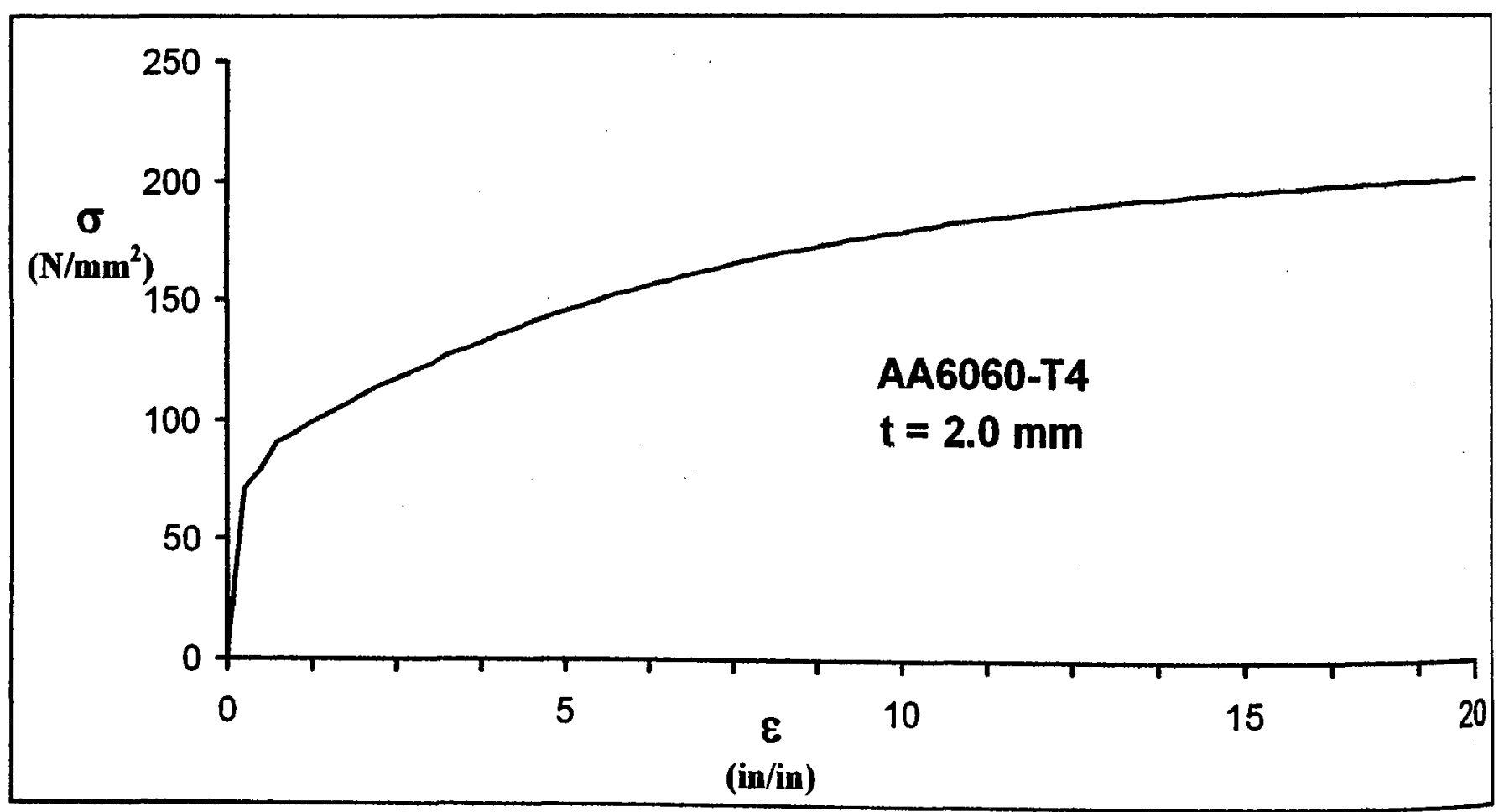

Figure 3.7 - True stress-strain curve of AA6060-T4 alloy $\mathrm{t}=2.0 \mathrm{~mm}$. 


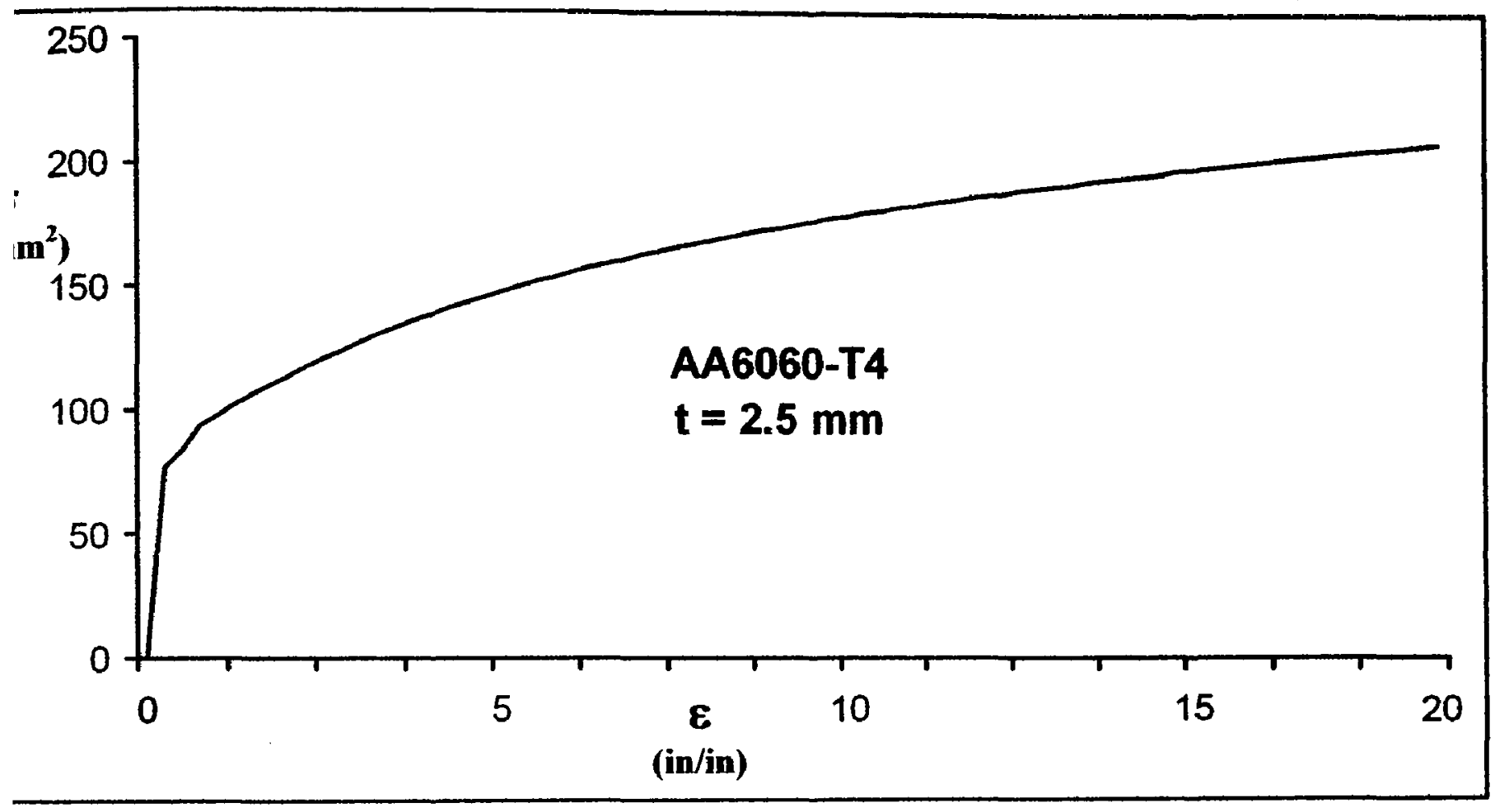

Figure 3.8 - True stress-strain curve of AA6060-T4 alloy $\mathrm{t}=2.5 \mathrm{~mm}$. 


\subsection{MODEL MESH ELEMENTS}

All the extruded tubes were modelled using Belytschko-Lin-Tsay 4-node shell elements with nine integration points through the thickness and only one integration point in the element plane. The optimal mesh density for all the models was determined to be in the order of $4 \mathrm{~mm} \times 4 \mathrm{~mm}$.

The Belytschko-Lin-Tsay shell element is the default shell element for computation formulation in LS-DYNA superseding the Hughes-Liu shell element. For example, a given shell element with five through-the-thickness integration points would require 725 mathematical operations for a Belytschko-Lin-Tsay shell element compared to 35,367 operations for the selectively reduced integration formulation of the Hughes-Liu shell element. As shown, the Belytschko-Lin-Tsay shell element is much more computational efficient without sacrificing accuracy.

The Belytschko-Lin-Tsay shell element is based on a combined co-rotational and velocity-strain formulation. The efficiency of the element is obtained from the mathematical simplifications that result from these two kinematical assumptions. The corotational portion of the formulation avoids the complexities of non-linear mechanics by embedding a coordinate system in the element. The choice of velocity strain, or rate of deformation in the formulation helps the constitutive evaluation since the conjugate stress is the more familiar Cauchy stress [27]. 


\subsubsection{BELYTCHKO-LIN-TSAY SHELL ELEMENT CO- ROTATIONAL COORDINATES}

The midsurface of the quadrilateral shell element, or reference surface, is defined by the location of the element's four comer nodes. An embedded element coordinate system that deforms with the element is defined in terms of these nodal coordinates. The procedure for constructing the co-rotational coordinate system begins by calculating a unit vector normal to the main diagonal of the element (see Figure 3.9):

$$
\begin{gathered}
\hat{e}_{3}=\frac{s_{3}}{\left\|s_{3}\right\|} \\
\left\|s_{3}\right\|=\sqrt{s_{31}^{2}+s_{32}^{2}+s_{33}^{2}} \\
s_{3}=r_{31} \times r_{42}
\end{gathered}
$$

where the superscript $(\wedge)$ represents the local element coordinate system. The local element $\mathbf{x}$-axis $(\hat{\mathbf{x}})$ is established approximately along the element edge between nodes 1 and 2 which is convenient for interpreting the element stresses which are defined in the local element $\hat{x}-\hat{y}$ coordinate system (see Figure 3.9).

The required procedure for constructing this unit vector involves defining a vector $s_{1}$ that is nearly parallel to the vector $\mathrm{I}_{21}$ where:

$$
\begin{aligned}
& s_{1}=r_{21}-\left(r_{21} \cdot \hat{e}_{3}\right) \hat{e}_{3} \\
& \hat{e}_{1}=\frac{s_{1}}{\left\|s_{1}\right\|}
\end{aligned}
$$

efore, the remaining undefined unit vector is determined from the vector cross uct:

$$
\hat{\mathbf{e}}_{2}=\hat{\mathbf{e}}_{3} \times \hat{\mathbf{e}}_{1}
$$




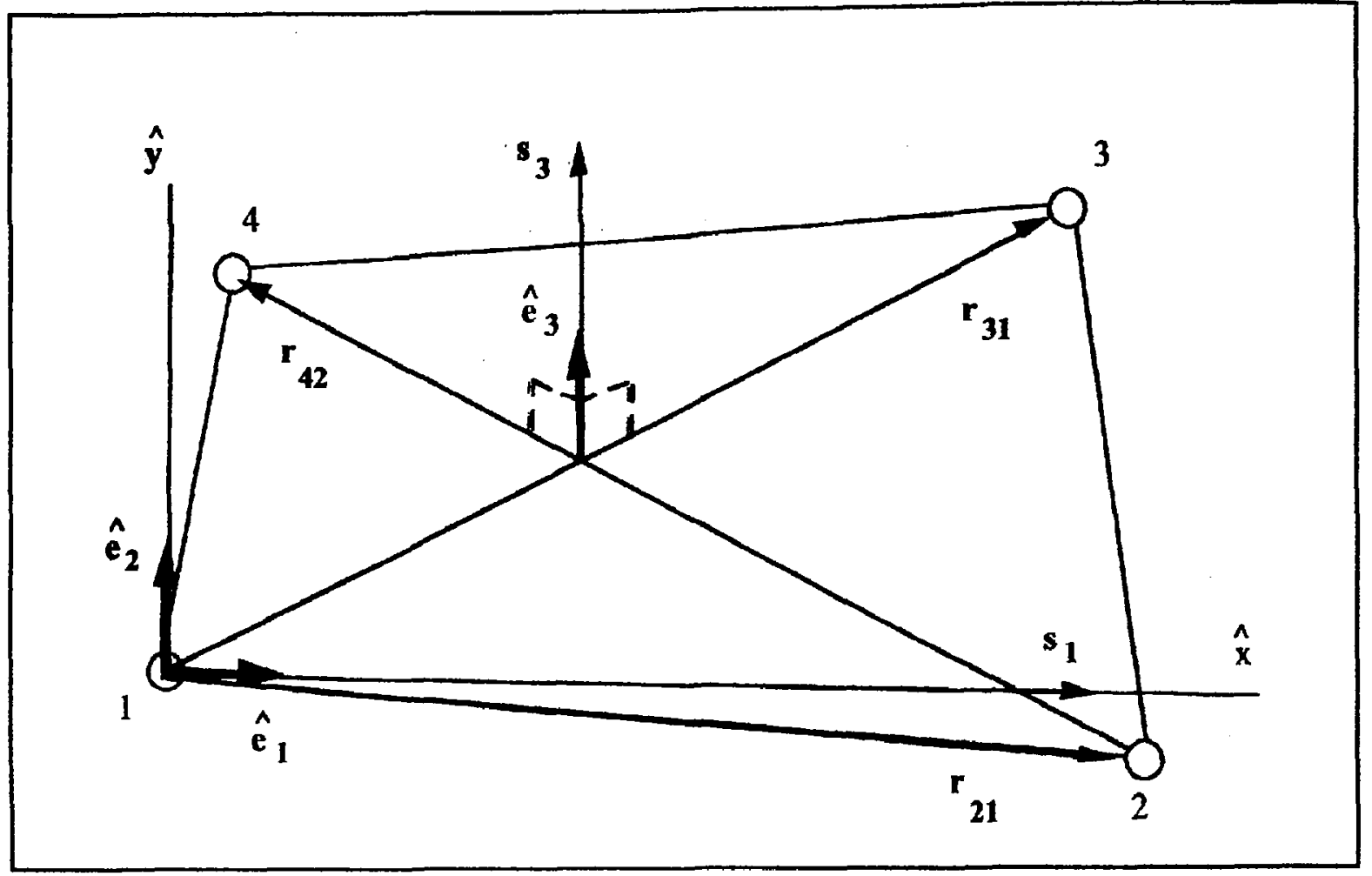

Figure 3.9 - Element coordinate system parameters [27].

In the case where the four nodes of the element are coplanar, then the unit vectors $\hat{\mathrm{e}}_{1}$ and $\hat{\mathrm{e}}_{2}$ are tangent to the mid-plane of the shell and $\hat{\mathrm{e}}_{3}$ is in the fibre direction. During the element deformation, an angle may develop between the actual fibre and the unit normal $\hat{\mathbf{e}}_{3}$. The magnitude of this angle may be characterized as:

$$
\left|\hat{\mathbf{e}}_{3} \cdot f-1\right|<\delta
$$

where $f$ is the unit vector in the fibre direction and the magnitude of $\delta$ depends on the magnitude of the strains. For most engineering applications according to Belytschko, acceptable values of $\delta$ are on the order of $10^{-2}$ and if the condition defined in Equation (3.10) is met, then the difference between the rotation of the co-rotational coordinates $\hat{e}$ and the material rotation should be small (negligible). 
The global components of the co-rotational unit vectors $\left(\hat{\mathrm{e}}_{1}, \hat{\mathrm{e}}_{2}, \hat{\mathbf{e}}_{3}\right)$ are ultimately used to define a transformation matrix between the global and local element coordinate systems. 


\subsubsection{VELOCITY-STRAIN DISPLACEMENT RELATIONS}

The small rotation condition characterized by Equation (3.10) does not restrict the magnitude of the element's rigid body rotations [27]. Instead, the restriction is placed on the out-of-plane deformations, and thus on the element strain. The displacement of any point in the shell is partitioned into a mid-surface displacement (nodal translations) and also a displacement associated with rotations of the element's fibres (nodal rotations). The Mindlin theory of plates and shells is used by the Belytschko-Lin-Tsay shell elements to partition the velocity of any point in the shell using the following expression:

$$
v=v^{m}-\hat{z} \hat{e}_{3} \times \theta
$$

where $v^{m}$ is the velocity of the mid-surface, $\theta$ is the angular velocity vector, and $\hat{z}$ is the distance along the fibre direction (thickness) of the shell element. The corresponding corotational components of the velocity strains (rate of deformation) are given by:

$$
\hat{\mathrm{d}}_{\mathrm{ij}}=\frac{1}{2}\left(\frac{\partial \hat{\mathrm{v}}_{\mathrm{i}}}{\partial \hat{\mathrm{x}}_{\mathrm{j}}}+\frac{\partial \hat{\mathrm{v}}_{\mathrm{j}}}{\partial \hat{\mathrm{x}}_{\mathrm{i}}}\right)
$$

The following velocity-strain relations were obtained by substituting Equation (3.11) into Equation (3.12):

$$
\begin{aligned}
& \hat{\mathrm{d}}_{\mathrm{x}}=\left(\frac{\partial \hat{\mathrm{d}}_{\mathrm{s}}^{\mathrm{m}}}{\partial \hat{\mathrm{x}}}+\hat{\mathrm{z}} \frac{\partial \hat{\theta}_{\mathrm{y}}}{\partial \hat{\mathrm{x}}}\right) \\
& \hat{\mathrm{d}}_{\mathrm{y}}=\left(\frac{\partial \hat{\mathrm{d}}_{\mathrm{y}}^{\mathrm{m}}}{\partial \hat{\mathrm{y}}}-\hat{\mathrm{z}} \frac{\partial \hat{\theta}_{\mathrm{x}}}{\partial \hat{\mathrm{y}}}\right)
\end{aligned}
$$




$$
\begin{gathered}
2 \hat{\mathrm{d}}_{\mathrm{xy}}=\left(\frac{\partial \hat{\mathrm{v}}_{\mathrm{s}}^{\mathrm{m}}}{\partial \hat{\mathrm{y}}}+\frac{\partial \hat{\mathrm{v}}_{\mathrm{y}}^{\mathrm{m}}}{\partial \hat{\mathrm{x}}}\right)+\hat{\mathrm{z}}\left(\frac{\partial \hat{\theta}_{\mathrm{y}}}{\partial \hat{\mathrm{y}}}-\frac{\partial \hat{\theta}_{\mathrm{x}}}{\partial \hat{\mathrm{x}}}\right) \\
2 \hat{\mathrm{d}}_{\mathrm{yz}}=\frac{\partial \hat{\mathrm{d}}_{\mathrm{z}}^{\mathrm{m}}}{\partial \hat{\mathrm{y}}}-\hat{\theta}_{\mathrm{x}} \\
2 \hat{\mathrm{d}}_{\mathrm{xz}}=\frac{\partial \hat{\mathrm{d}}_{\mathrm{z}}^{\mathrm{m}}}{\partial \hat{\mathrm{x}}}+\hat{\theta}_{\mathrm{y}}
\end{gathered}
$$

The derived above velocity-strain relations require to be evaluated at the quadrature points within the shell. This can be accomplished using standard bilinear nodal interpolation that is used to define the mid-surface velocity, angular velocity, and also the element's coordinates. Expressions for these interpolations are defined as:

Mid-surface Velocity: $\quad v^{m}=N_{I}(\xi, \eta) v_{I}$

Mid-surface Angular Velocity: $\quad \theta^{\mathrm{m}}=\mathrm{N}_{\mathrm{I}}(\xi, \eta) \theta_{\mathrm{I}}$

Mid-surface Coordinates:

$$
x^{m}=N_{I}(\xi, \eta) x_{I}
$$

Where the subscript I is summed over all the element's nodes and the nodal velocities are obtained by differentiating the nodal coordinates with respect to time which yields the following bilinear shape functions:

$$
\begin{aligned}
& N_{1}=1 / 4(1-\xi)(1-\eta) \\
& N_{2}=1 / 4(1+\xi)(1-\eta) \\
& N_{3}=1 / 4(1+\xi)(1+\eta) \\
& N_{4}=1 / 4(1-\xi)(1+\eta)
\end{aligned}
$$


The velocity strains at the center of the element where $\xi=0$ and $\eta=0$ are obtained by substitution of the above relations into the previously defined velocity-strain displacement relations (Equations (3.13) to (3.17)) which with some algebraic manipulation yield:

$$
\begin{gathered}
\hat{d}_{x}=B_{1 I} \hat{v}_{x I}+\hat{z} B_{1 I} \hat{\theta}_{y I} \\
\hat{d}_{y}=B_{2 I} \hat{v}_{y I}+\hat{z} B_{2 I} \hat{\theta}_{x I} \\
2 \hat{d}_{x y}=B_{2 I} \hat{v}_{x I}+B_{1 I} \hat{v}_{y I}+\hat{z}\left(B_{2 I} \hat{\theta}_{y I}-B_{1 I} \hat{\theta}_{x I}\right) \\
2 \hat{d}_{x z}=B_{1 I} \hat{v}_{z I}+N_{I} \hat{\theta}_{y I} \\
2 \hat{d}_{y z}=B_{2 I} \hat{v}_{z I}-N_{I} \hat{\theta}_{x I}
\end{gathered}
$$

where

$$
\begin{aligned}
& \mathrm{B}_{1 \mathrm{I}}=\frac{\partial \mathrm{N}_{\mathrm{I}}}{\partial \hat{\mathrm{x}}} \\
& \mathrm{B}_{2 \mathrm{I}}=\frac{\partial \mathrm{N}_{\mathrm{I}}}{\partial \hat{\mathrm{y}}}
\end{aligned}
$$

Similarly to the velocity strains, the shape function derivatives $B_{1 I}$ and $B_{2 I}$ are also evaluated at the center of the element where $\xi=0$ and $\eta=0$. 


\subsubsection{STRESS RESULTANTS AND NODAL FORCES}

After evaluating the velocity strains, the resulting stresses are integrated through the thickness of the shell to obtain local resultant forces and moments. The integration formulae for the resultants are as follows:

Resultant Force: $\quad \hat{\mathrm{f}}_{\alpha \beta}^{\mathrm{R}}=\int \hat{\sigma}_{\alpha \beta} d \hat{z}$

Resultant Moment: $\quad \hat{\mathbf{m}}_{\alpha \beta}^{\mathrm{R}}=-\int \hat{z} \hat{\sigma}_{\alpha \beta} \mathrm{d} \hat{\mathbf{z}}$

where the $\mathbf{R}$ superscript represents a resultant force or moment and the Greek subscripts $\alpha$ and $\beta$ indicate the limited range of the indices for plane stress plasticity. The resultant force and moment are element centred and are related to the local nodal forces and moments by the use of the principle of virtual power and performing a one-point quadrature. As a result, the following relations are obtained:

$$
\begin{gathered}
\hat{\mathrm{f}}_{\mathrm{xI}}=\mathrm{A}\left(\mathrm{B}_{1 \mathrm{I}} \hat{\mathrm{f}}_{\mathrm{xx}}^{\mathrm{R}}+\mathrm{B}_{2 \mathrm{I}} \hat{\mathrm{f}}_{\mathrm{xy}}^{\mathrm{R}}\right) \\
\hat{\mathbf{f}}_{\mathrm{yI}}=\mathrm{A}\left(\mathrm{B}_{2 \mathrm{I}} \hat{\mathrm{f}}_{\mathrm{yy}}^{\mathrm{R}}+\mathrm{B}_{1 \mathrm{f}} \hat{\mathrm{f}}_{\mathrm{xy}}^{\mathrm{R}}\right) \\
\hat{\mathrm{f}}_{\mathrm{zI}}=\mathrm{Ak}\left(\mathrm{B}_{1 \mathrm{II}} \hat{\mathrm{f}}_{\mathrm{xz}}^{\mathrm{R}}+\mathrm{B}_{2 \mathrm{f}} \hat{\mathrm{f}}_{\mathrm{yz}}^{\mathrm{R}}\right) \\
\hat{\mathrm{m}}_{\mathrm{xI}}=\mathrm{A}\left(\mathrm{B}_{2 \mathrm{I}} \hat{\mathrm{m}}_{\mathrm{yy}}^{\mathrm{R}}+\mathrm{B}_{1 \mathrm{I}} \hat{\mathrm{m}}_{\mathrm{xy}}^{\mathrm{R}}-\frac{\mathrm{K}}{4} \hat{\mathrm{f}}_{\mathrm{yz}}^{\mathrm{R}}\right) \\
\hat{\mathrm{m}}_{\mathrm{yI}}=\mathrm{A}\left(\mathrm{B}_{1 \mathrm{I}} \hat{\mathrm{m}}_{\mathrm{xx}}^{\mathrm{R}}+\mathrm{B}_{2 \mathrm{I}} \hat{\mathrm{m}}_{\mathrm{xy}}^{\mathrm{R}}-\frac{\mathrm{k}}{4} \hat{\mathrm{f}}_{\mathrm{xz}}^{\mathrm{R}}\right) \\
\hat{\mathrm{m}}_{\mathrm{zI}}=0
\end{gathered}
$$


where $\mathrm{A}$ is the area of the element and $\mathrm{k}$ is the shear factor from the Mindlin theory. In the Belytschko-Lin-Tsay element formulation, $K$ is utilized to enforce the Kirchhoff normality condition and serves as a penalty parameter as the shell becomes thin [27].

The local nodal forces and moments are then transformed to the global coordinate system using the similar transformation relations used for the co-rotational unit vectors $\left(\hat{\mathrm{e}}_{1}, \hat{\mathrm{e}}_{2}, \hat{\mathrm{e}}_{3}\right)$. Upon the transformation to the global coordinate system, the global nodal forces and moments are summed over all the nodes and the global equations of motion are solved for the next increment in nodal accelerations. 


\subsection{CONTACT-IMPACT ALGORITHM}

The powerful capability of the LS-DYNA code to model sliding and impact non-linear behaviour very efficiently made it the most suitable tool to serve the finite element analysis code to evaluate the impact energy dissipation of the complex tube sections. LSDYNA uses three distinct methods for handling impact along interfaces: (1) Kinematic Constraint Method, (2) The Penalty Method, and (3) The Distributed Parameter Method [27].

The contact algorithms evaluated for the impact numerical simulations were both the CONTACT_AUTOMATIC_SINGLE_SURFACE and CONTACT_AUTOMATIC _SURFACE_TO_SURFACE where both make use of the Penalty Method (PM). Based on the initial results of the dynamic impact numerical simulations, it was noticed that both contact algorithms showed very little difference in the results, which made sense since the single surface contact algorithm evolved from the surface to surface contact algorithm. Therefore, the CONTACT_AUTOMATIC_SINGLE_SURFACE was used more extensively due to the input deck being less laborious to set-up between code executions.

The contact algorithm basically allows interfaces to be defined in three dimensions by listing an arbitrary order to the elements that comprise each side of the interface. One side of the interface is designated as the slave side, and the other is designated as the master side. The element nodes located in those surfaces are referred to as slave and master nodes, respectively. For the PM, this distinction is irrelevant, but in the other methods the slave nodes are constrained to slide on the master surface after impact and must remain on the master surface until a tensile force develops between the node and the surface. The antnmatic contact definitions (i.e. CONTACT_AUTOMATIC_SINGLE_SURFACE), as that used for the numerical simulations contained in this report, facilitate the ess and make it more transparent. This approach allows the slave and master surfaces e generated automatically within LS-DYNA from the part identification numbers n for each surface of the model. Some automotive manufacturers for their crash 
models include the entire vehicle in one single surface contact definition where all the nodes and elements are automatically interact within the interface. In the case of large complex models, this allows considerable time and labour to be saved in preparing the models [27].

\subsubsection{THE PENALTY METHOD (PM)}

The PM essentially consists of placing normal interface springs between all penetrating nodes and the contact surface. As a result, the spring stiffness matrix developed by the use of the PM is automatically incorporated into the global stiffness matrix. Compared to other nodal constraint methods, the PM approach excites very little hourglassing effects of the elements which can be directly attributed to the symmetry of the approach. This allows momentum to be exactly conserved without the necessity of imposing additional impact and release conditions.

\subsubsection{THE SLAVE SEARCH PROCESS}

The slave search finds the nearest point on the master surface for each slave node. Lines drawn from a slave node to its nearest point will be perpendicular to the master surface, unless the point lies along the intersection of two master segments, where the segment is defined to be a 3- or 4-node element of a surface. Consider a slave node, $\mathbf{n}_{\mathbf{s}}$, sliding on a piecewise smooth master surface and assume that a search of the master surface has located the master node, $m_{s}$, lying nearest to $n_{s}$ (see figure 3.10). If $m_{s}$ and $n_{s}$ do not coincide, $\mathbf{n}_{\mathbf{s}}$ can usually be shown to lie in a segment $\mathbf{s}_{\mathbf{i}}$ via the following tests: 


$$
\begin{aligned}
& \left(c_{i} \times s\right) \cdot\left(c_{i} \times c_{i+1}\right)>0 \\
& \left(c_{i} \times s\right) \cdot\left(s \times c_{i+1}\right)>0
\end{aligned}
$$

where vector $c_{1}$ and $c_{+1}$ are along edges of $s_{i}$ and point outward from $m_{1}$ (see Figure 3.11). Vector $s$ is the projection of the vector beginning at $\mathbf{m}_{3}$, ending at $n_{\mathbf{z}}$, and denoted by $g$ on the plane being examined.

$$
\mathrm{s}=\mathrm{g}-(\mathrm{g} \cdot \mathrm{m}) \mathrm{m}
$$

where for segment $s_{i}$ : $\quad m=\frac{c_{i} \times c_{i+1}}{\left|c_{i} \times c_{i+1}\right|}$

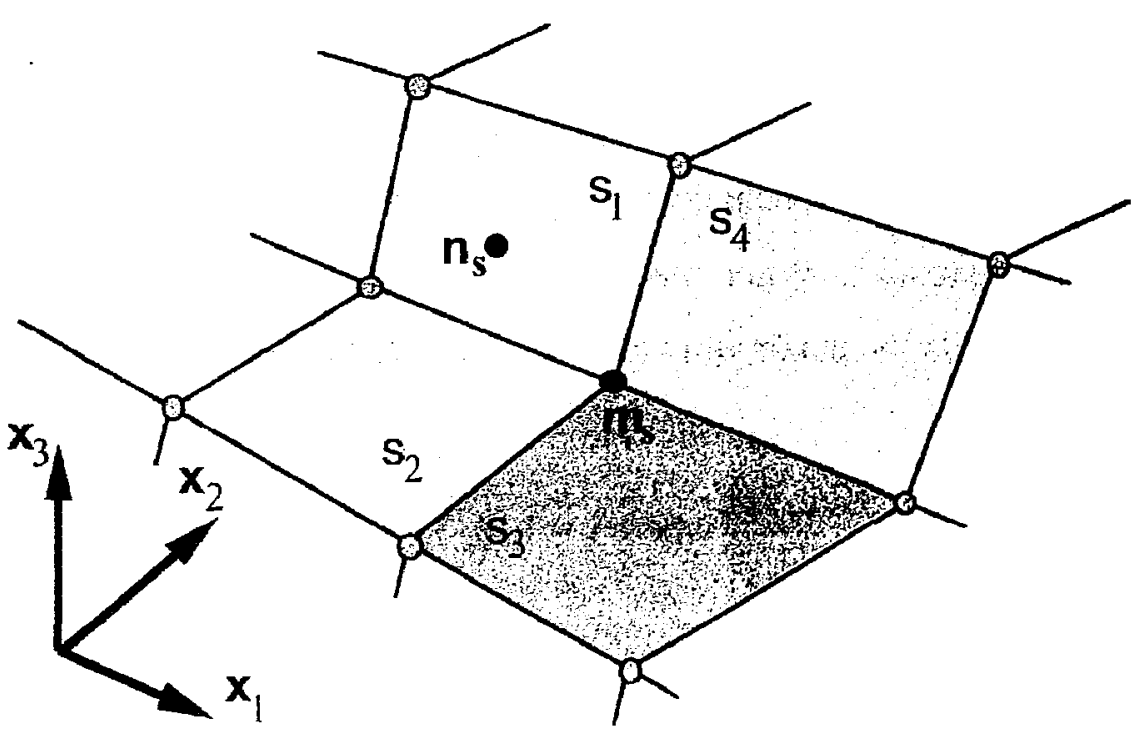

re 3.10 - Four master segments can harbour slave node $\mathrm{n}_{\mathbf{8}}$ given that $\mathrm{m}_{\mathbf{8}}$ is shown the nearest master mode as shown above [27]. 


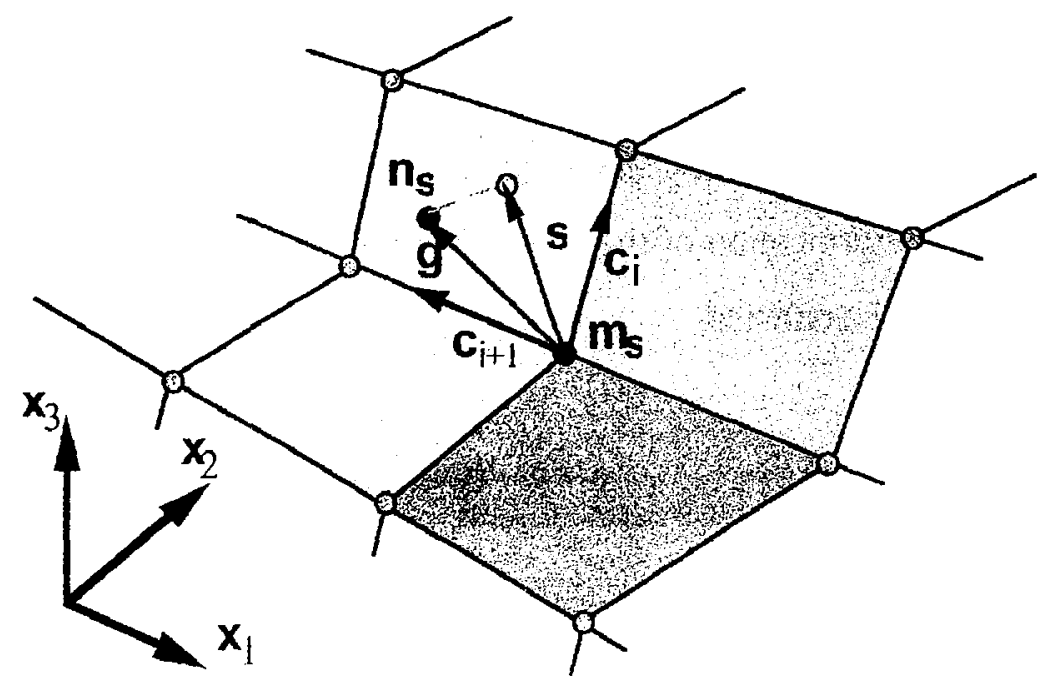

Figure 3.11 - Projection of $\mathbf{g}$ onto the master segment $\mathrm{s}_{\mathbf{i}}[27]$.

Since the sliding constraints keep $\mathbf{n}_{\mathbf{s}}$ close but not necessarily on the master surface and since $\mathbf{n}_{\mathbf{s}}$ may lie near or even on the intersection of two master segments, the inequalities of Equations (3.40) may be inconclusive, i.e. they may fail to be satisfied or more than one may give positive results. When this occurs, $\mathbf{n}_{\mathbf{s}}$ is assumed to lie along the intersection which yields the maximum value for the quantity:

$$
\frac{c_{i} \cdot g}{\left|c_{i}\right|} \text { where } i=1,2,3,4, \ldots
$$

Assuming that a master segment has been located for slave node $\mathbf{n}_{\mathbf{s}}$ and that $\mathbf{n}_{\mathbf{s}}$ is not identified as lying on the intersection of two master segments, then the identification of the contact point becomes non-trivial. As a result, each master surface segment, $s_{i}$, is given the parametric representation of:

$$
r=f_{1}(\xi, \eta) i_{1}+f_{2}(\xi, \eta) i_{2}+f_{3}(\xi, \eta) i_{3}
$$


where

$$
\mathbf{f}_{\mathbf{i}}(\xi, \eta)=\sum_{\mathbf{j}=1}^{4} \phi_{\mathbf{j}} \mathbf{x}_{\mathbf{i}}^{\mathbf{j}}
$$

and noting that $\mathbf{r}_{\mathbf{i}}$ is at least once continuously differentiable with the contact point centre coordinates $\left(\xi_{c}, \eta_{c}\right)$ :

$$
\frac{\partial \mathrm{r}}{\partial \xi} \times \frac{\partial \mathrm{r}}{\partial \eta} \neq 0
$$

Therefore, $r$ represents a master segment that has a unique normal whose direction depends continuously on the points of master segment $\mathbf{s}_{\mathrm{i}}$. Letting $\mathbf{t}$ be a position vector drawn to slave node $n_{s}$ and assuming that the master surface segment $s_{i}$ has been identified with $\mathbf{n}_{\mathrm{s}}$, the contact point coordinates $\left(\xi_{c}, \eta_{c}\right)$ on $\mathbf{s}_{\mathbf{i}}$ satisfy the following criteria (see Figure 3.12):

$$
\begin{aligned}
& \frac{\partial}{\partial \xi}\left(\xi_{c}, \eta_{c}\right)\left[t-r\left(\xi_{c}, \eta_{c}\right)\right]=0 \\
& \frac{\partial}{\partial \eta}\left(\xi_{c}, \eta_{c}\right)\left[t-r\left(\xi_{c}, \eta_{c}\right)\right]=0
\end{aligned}
$$

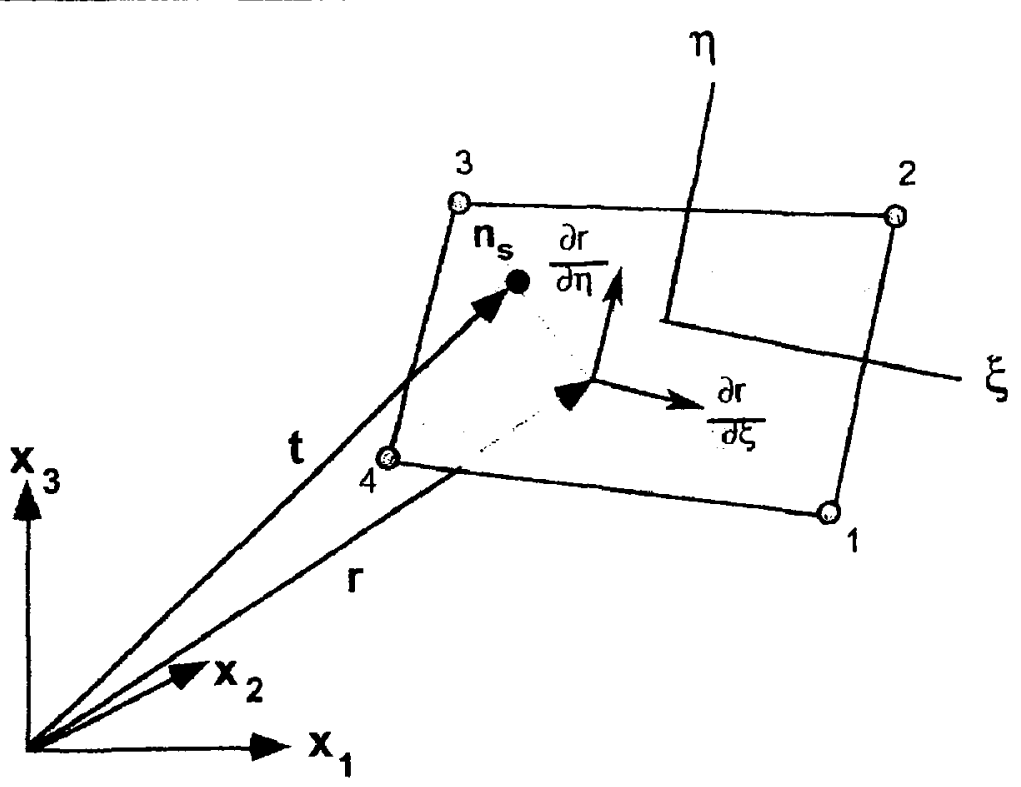

igure 3.12 - Location of contact point when $n_{s}$ lies above the master segment [16]. 
Equations (3.47) and (3.48) can be numerically solved for $\xi_{c}$ and $\eta_{c}$ using a NewtonRaphson iteration that easily accommodates the simple non-linear equations. The method diverges with distorted elements unless the initial guess is accurate. An exact contact point calculation is critical in post-buckling calculations which is directly applicable to the results from the various tube sections validated. The exact contact point calculation ensures that the solution does not wander away from the desired buckling mode. Three iterations with a least-squares projection are used to generate an initial guess followed by the Newton-Raphson iterations which are limited to ten iterations, but typically converges in four or less [27].

Since the PM is applied, each slave node is checked for penetration through the master surface. If there is no penetration, nothing occurs at the slave node and contact point. If penetration does occur, an interface force is applied between the slave node and its contact point. The magnitude of this force is proportional to the amount of penetration and can be idealized as the addition of a spring at the interface.

Penetration of the slave node $\mathbf{n}_{\mathbf{s}}$ through the master segment which contains its contact point is denoted by the parameter $\Delta \mathrm{l}$ where:

$$
\Delta l=n_{i} \cdot\left[t-r\left(\xi_{c}, \eta_{c}\right)\right]<0
$$

where

$$
\left.n_{i}=n_{i}\left(\xi_{c}, \eta_{c}\right)\right]
$$

is the normal vector to the master segment at the contact point.

The interface force vector resulting from the slave node $\mathbf{n}_{\mathbf{s}}$ penetration through the master segment $\boldsymbol{s}_{\mathbf{l}}$ is defined by $\mathbf{f}_{\mathbf{s}}$ :

$$
f_{s}=-\Delta l k_{i} \cdot n_{i} \text { if } \Delta l<0
$$


where:

$i=1,2,3,4$ - four nodes of slave node $n_{i}$

$k_{i}=$ stiffiness factor for master segment $s_{i}=\frac{f_{s i} K_{i} A_{i}}{\max (\text { shell diagonal) }}$

$\mathrm{K}_{\mathbf{i}}=$ bulk modulus of element that contains master segment $\mathbf{s}_{\mathbf{i}}$

$V_{i}=$ volume of element that contains master segment $s_{i}$

$A_{i}=$ face area of element that contains master segment $s_{i}$

$\mathrm{f}_{\mathrm{si}}=$ scale factor for interface stiffness and default value is 0.10

Accounting for the degrees of freedom corresponding to the slave node $n_{\mathbf{s}}$ and the four nodes that comprise the master segment $\mathrm{s}_{\mathrm{i}}$, the interface force can be further expressed as:

$$
\left.\mathrm{f}_{\mathrm{m}}^{\mathrm{i}}=\phi_{\mathrm{i}}\left(\xi_{\mathrm{c}}, \eta_{\mathrm{c}}\right)\right] \mathrm{f}_{\mathrm{s}}
$$

The interface force can be used to determine the contact energy, $E_{\text {contact, }}$ which is incrementally updated from time $\mathbf{n}$ to time $\mathbf{n}+\mathbf{1}$ for each contact interface:

$$
E_{\text {contact }}^{n+1}=E_{\text {contact }}^{n}+\left[\sum_{i=1}^{n s n} \Delta F_{i}^{\text {slave }} \times \Delta \text { dist }_{i}^{\text {slave }}+\sum_{i=1}^{n m m} \Delta F_{i}^{\text {masser }} \times \Delta \text { dist }_{i}^{\text {master }}\right]^{n+\frac{1}{2}}
$$

where:

nsn $=$ number of slave nodes

$\mathrm{nmn}=$ number of master nodes

$\Delta \mathrm{F}_{\mathrm{i}}^{\text {slave }}=\quad$ the interface force between the $i$ th slave node and the contact segment.

$\Delta$ dist $_{\mathbf{i}}^{\text {slave }}=$ the incremental distance the $i$ th slave node has moved during the current time step.

naster $=$ the interface force between the $i$ th master node and the contact segment.

$\mathbf{s t}_{\mathbf{i}}^{\text {master }}=$ the incremental distance the $i$ th master node has moved during the current time step. 
In the absence of friction, the slave and master side energies should be close in magnitude but opposite in sign. The sum, $E_{\text {contact, }}$ should equal the stored energy under normal conditions, but large negative values are indicative of undetected penetrations and thus should be monitored. 


\section{Chapter 4}

\section{VALIDATION OF ANALYSIS}

When using a computational model to analyze a physical phenomenon, it is crucial to check the validity of that model, preferably with measured data from experimental tests. This was the primary objective of the first phase of the research study for the LS-DYNA numerical simulations. The validation criterion was defined as the correlation of the LSDYNA numerical simulations to those of published experimental results pertaining to Langseth and Hopperstad (Department of Structural Engineering, Norwegian University of Science and Technology) [5]. More specifically, the experimental results from their work involving the dynamic axial crushing of thin-walled aluminium alloy extruded square tubes were to be established as the baseline conditions that the LS-DYNA simulations were to predict with a reasonable degree of accuracy. The primary response parameters adopted to gauge the validation of the numerical results with the experimental data consisted of the mean axial crush load $\left(P_{m d}\right)$ and the permanent axial displacement of the collapsed member $\left(\delta_{\mathrm{p}}\right)$ which are typically used to evaluate the energy dissipation capability of structural components. The post-buckling deformation characteristics of the axially crushed members were also to be compared to ensure that there was good agreement. 
Table 4.I- Experimental results compared to LS-DYNA numerical simulation results.

\begin{tabular}{|c|c|c|c|c|c|c|}
\hline \multirow[b]{2}{*}{$\begin{array}{l}\text { Experimental } \\
\text { Specimen }\end{array}$} & \multicolumn{2}{|c|}{$\begin{array}{c}\text { Experimental } \\
\text { Test Results } \\
\end{array}$} & \multicolumn{2}{|c|}{$\begin{array}{c}\text { LS-DYNA } \\
\text { Results } \\
\end{array}$} & \multicolumn{2}{|c|}{$\begin{array}{l}\text { Percentage } \\
\text { Difference } \\
\end{array}$} \\
\hline & $\begin{array}{c}\delta_{\mathrm{p}} \\
(\mathrm{mm})\end{array}$ & $\begin{array}{c}\mathbf{P}_{\mathbf{m d}} \\
(\mathbf{K N}) \\
\end{array}$ & $\begin{array}{c}\delta_{p} \\
(\mathbf{m m})\end{array}$ & $\begin{array}{c}\mathbf{P}_{\mathbf{m d}} \\
(\mathrm{KN})\end{array}$ & $\begin{array}{l}\Delta \delta_{p} \\
(\%) \\
\end{array}$ & $\begin{array}{l}\Delta \mathbf{P}_{\text {md }} \\
(\%)\end{array}$ \\
\hline EX1.8-1-SQ & 117.0 & 24.6 & 122.0 & 24.0 & -4.27 & 2.44 \\
\hline EX1.8-2-SQ & 129.0 & 21.8 & 130.0 & 21.9 & -0.78 & -0.46 \\
\hline EX1.8-3-SQ & 87.0 & 22.5 & 85.0 & 23.2 & 2.30 & -3.11 \\
\hline EX2.0-4-SQ & 126.0 & 29.3 & 128.0 & 29.3 & -1.59 & 0.00 \\
\hline EX2.0-5-SQ & 184.0 & 29.3 & 191.0 & 28.5 & -3.80 & 2.73 \\
\hline EX2.0-6-SQ & 82.0 & 29.7 & 81.0 & 30.4 & 1.22 & -2.36 \\
\hline EX2.5-7-SQ & 117.0 & 40.6 & 121.0 & 39.7 & -3.42 & 2.22 \\
\hline EX2.5-8-SQ & 73.0 & 46.0 & 75.0 & 45.3 & -2.74 & 1.52 \\
\hline EX2.5-9-SQ & 158.0 & 41.9 & 160.0 & 42.0 & -1.27 & -0.24 \\
\hline
\end{tabular}

With reference to Table 4.1 above, it can be seen that experimental values for both the mean axial crush load $\left(\mathrm{P}_{\mathrm{md}}\right)$ and permanent axial displacement $\left(\delta_{\mathrm{p}}\right)$ of the experimental specimen member were predicted within reasonable accuracy $( \pm 5 \%)$ by the LS-DYNA numerical simulations. It also seemed that in seven out of the nine specimens the numerical simulation tended to over predict the maximum displacement compared to the test data, but the same cannot be said for the mean axial crush load $\left(\mathbf{P}_{\mathbf{m d}}\right)$. The successful correlation of the experimental test specimens using LS-DYNA cannot only be attributed to the powerful capability of the LS-DYNA code to model impact non-linear behaviour very efficiently, but also the material property model adopted using the Five-Parameter Material Model (see Section 3.3) which was based on material properties obtained from experimental means by Langseth and Hopperstad. The actual evaluation of the material properties using experimental means allowed the ability to accurately define the material strain hardening effects and plasticity characteristics. In addition to using the FiveParameter Material Model, to obtain the true stress-strain curves of the AA6060-T4 material, the utilization of the LS-DYNA material card MAT_PIECEWISE_LINEAR PLASTICITY to define them for the numerical simulations contributed significantly to the overall accuracy of the results. This material card allows additional formulations for strain rate effects and also includes the flexibility to define stress-strain intervals with high degrees of plasticity and strain hardening effects more precisely. 


\subsection{POST-BUCKLING DEFORMATION}

The post-buckling deformation characteristics of the tube members predicted by the computational model can be considered as equally, if not more, important to the estimation of the mean axial crush load $\left(P_{m d}\right)$ and permanent axial displacement $\left(\delta_{p}\right)$. The ability to predict the various modes of the lobe formation (i.e., Type I, Type II, etc.) is paramount since it helps to ascertain their effect in the overall energy absorption of the member and as a result has an impact on the crashworthiness parameters mean axial crush load $\left(\mathbf{P}_{\mathbf{m d}}\right)$ and permanent axial displacement $\left(\delta_{\mathrm{p}}\right)$. Abramowicz and Jones [12] have demonstrated that extensional modes (Type II) are associated with increased energy absorption when compared to symmetric Type I or asymmetric mixed modes. Based on this, the introduction of symmetric Type II deformation modes are most ideal since they will yield the lease permanent deformation.

As a result, Langseth and Hopperstad [5] describe the post-buckling deformation characteristics of the experimental specimens with great detail. They observed that the post-buckling deformation modes of the experimental square tube specimens comprised of either symmetric or a combination of symmetric and asymmetric crush modes for the thinner specimens (i.e., $1.8 \mathrm{~mm}$ and $2.0 \mathrm{~mm}$ ). For the thicker $2.5 \mathrm{~mm}$ specimens, they noted only symmetric deformation modes. Also, they stated that the formation of the lobes started either at the impacted end of the specimens or at the clamped supported end and the extensional deformation (symmetric Type II) were always limited to either the first lobe or first two lobes from the impacted end. Furthermore, for low impact velocities, buckles with small amplitudes could be observed over the entire length of the specimens in addition to the localized lobes $[5,6]$.

As shall be demonstrated, their experimental observations were mirrored by the LSIA numerical results. For instance, Langseth and Hopperstad state that for the fic case of the $2.5 \mathrm{~mm}$ thick specimens in temper T4, only symmetric deformation is were present. The numerical results for the $2.5 \mathrm{~mm}$ thick specimens (LS2.5-7-SQ, 5-8-SQ, LS2.5-9-SQ) show that the post buckling deformation mode consisted of 
only symmetric type collapse as illustrated below in Figure 4.1 for a typical specimen (LS2.5-9-SQ shown).

Remaining lobe formations are symmetric Type I.

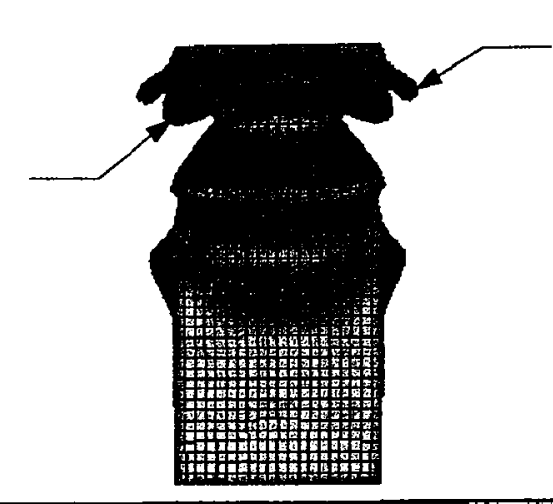

First lobe is extensional mode (symmetric Type II).

Figure 4.1 - Typical $2.5 \mathrm{~mm}$ thick specimen deformation characteristics

To further illustrate the good correlation for the post-buckling deformation characteristics of the LS-DYNA computational results to those of the experimental test results, an additional example is cited. Langseth and Hopperstad stated that for low impact velocities, buckles with small amplitudes could be observed over the entire length of the specimens in conjunction with the localized lobes resulting from the dynamic progressive buckling action. The same effect was found to have occurred when the numerical simulation results were studied and the deformation plots pertaining to the LS-DYNA models LS1.8-3-SQ $(\mathrm{V}=8.47 \mathrm{~m} / \mathrm{s})$ and LS2.0-6-SQ $(\mathrm{V}=9.45 \mathrm{~m} / \mathrm{s})$ are presented in Figure 4.2 to illustrate this effect.

Comparing the impact energy versus permanent axial displacement curve generated from the numerical simulation results with those of the experimental test data reveals good correlation. The trend is very similar with an almost linear relationship with the slope of the curve nearly identical to those generated by Langseth and Hopperstad based on their experimental results. Figure 4.3 and 4.4 represent the impact energy versus permanent axial displacement curve generated from the numerical simulations pertaining to the square tube section finite element models LS1.8-1-SQ and LS2.5-9-SQ, respectively. 


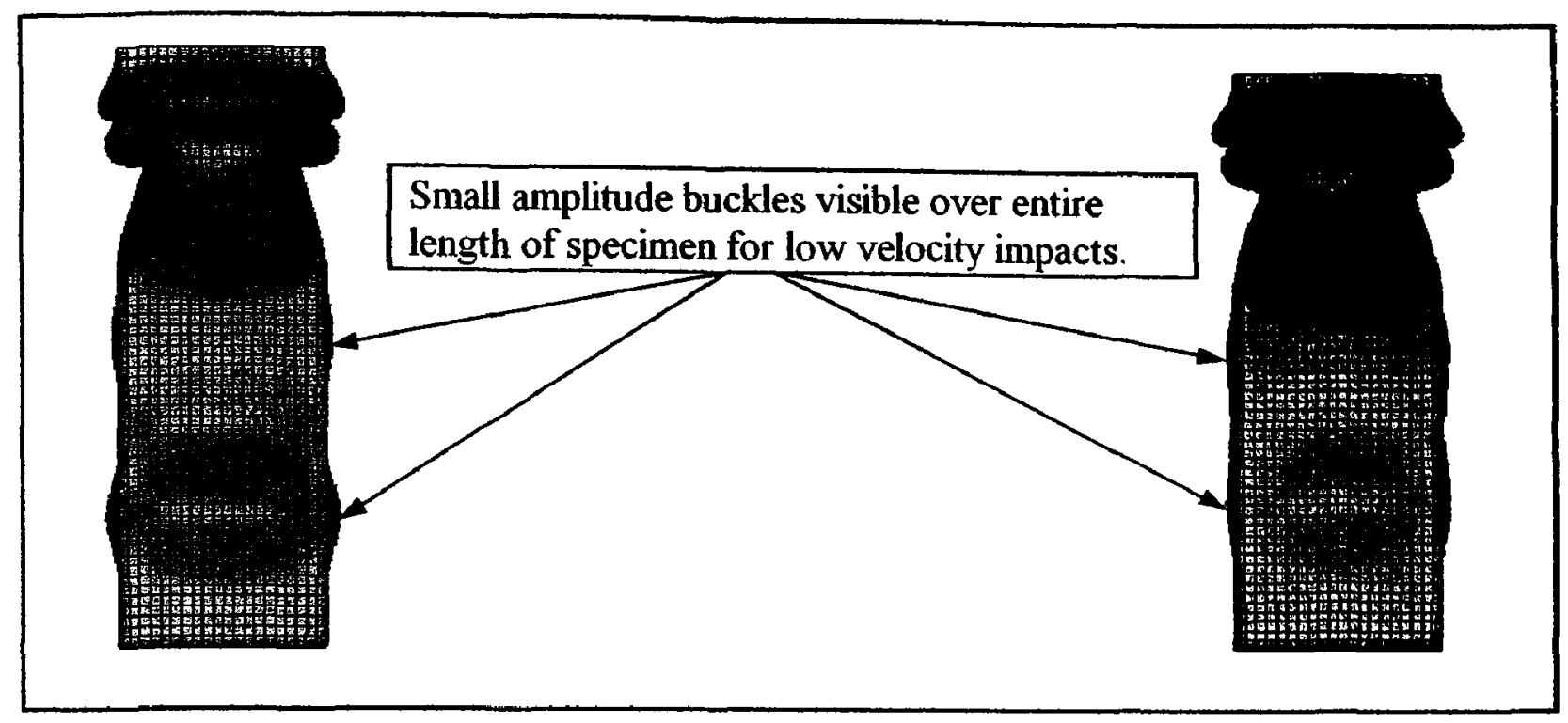

Figure 4.2 - Small amplitude buckling.

All of the remaining numerical simulation results pertaining to the square tube models displayed the almost linear relationship between the impact energy versus permanent axial displacement as presented in Figure 4.3 and 4.4.

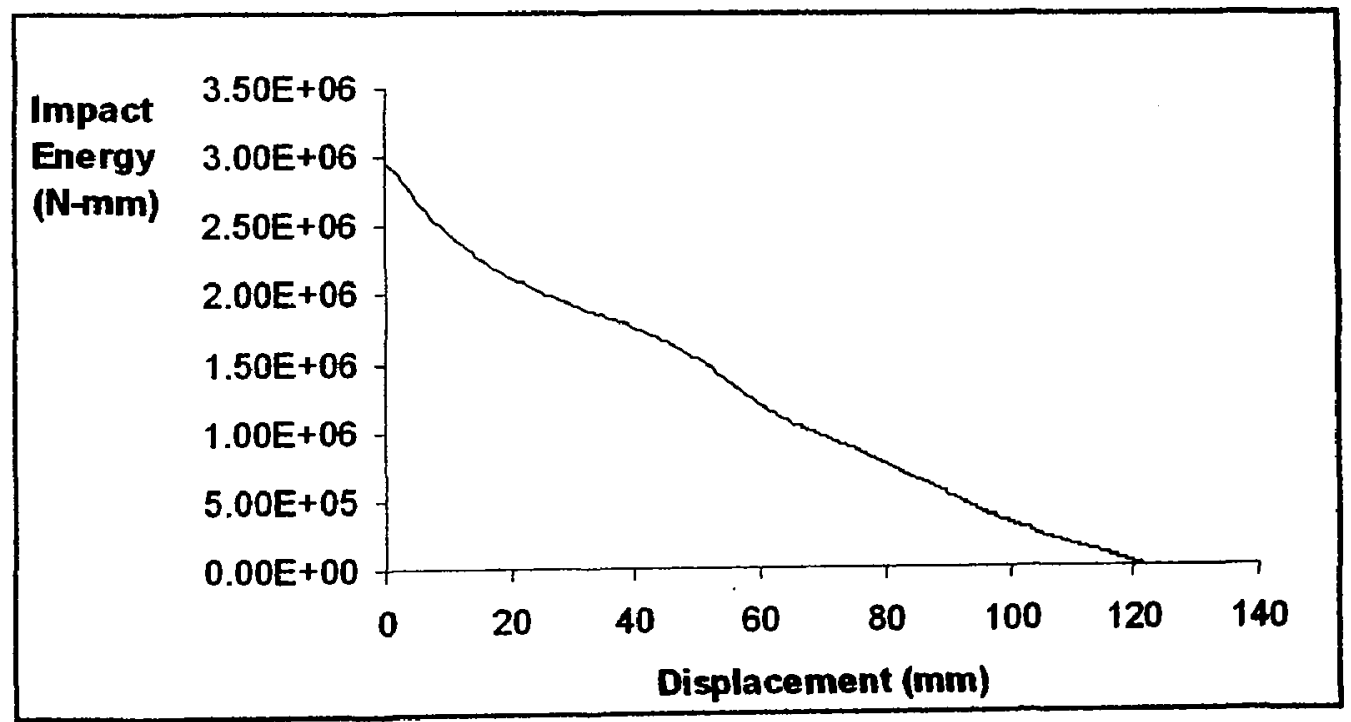

tre 4.3 - Impact Energy vs Displacement Curve of LS1.8-1-SQ numerical simulation. 


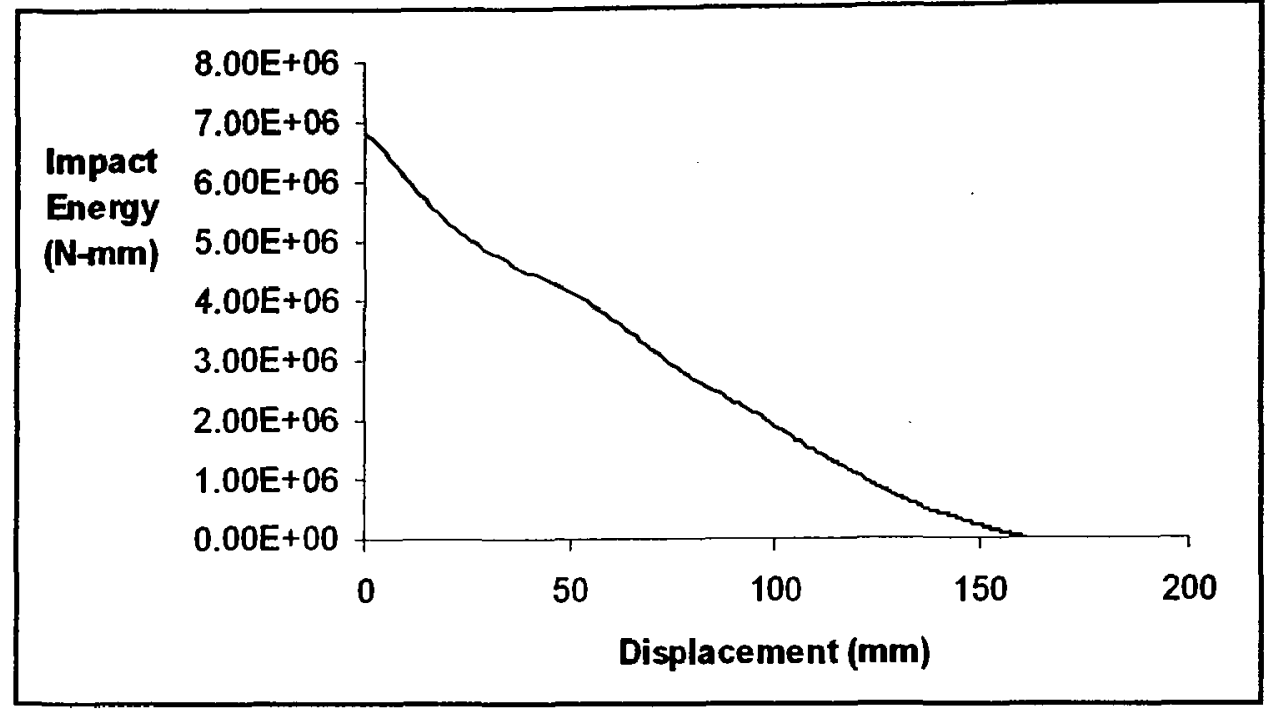

Figure 4.3 - Impact Energy vs Displacement Curve of LS2.5-9-SQ numerical simulation.

The good correlation achieved between the computational numerical and experimental results shall allow the second phase of the research study to be conducted with a fairly high degree of confidence. Furthermore, the square tube section specimens shall be used as a baseline to help assess the energy absorbing performance of the more complex hexagonal and octagonal sections. 


\section{Chapter 5}

\section{RESULTS AND DISCUSSION}

\subsection{NUMERICAL SIMULATION RESULTS}

LS-DYNA Version 960 was used for the computational analysis segment of this research study with the host computer being a desktop $2.4 \mathrm{GHz}$ Pentium 4 . The typical run time of a computational model was approximately 7 hours based 0.1 millisecond time steps.

Typical LS-DYNA post-buckling deformation plots derived from the numerical simulation results are presented in Figures 5.1 to 5.3 with each being representative of the tube sections researched. The remaining tube sections are presented in the Appendix A section of this report. A typical square tube section validated in the first phase of the research study is shown in Figure 5.1 where the post-buckling deformation plots are those pertaining to the LS1.8-1-SQ model. The post-buckling deformation plots show that the buckling lobe formations consist of a symmetric extensional Type II mode (first lobe formation from the top) and symmetric Type I modes (remaining lobes). Figure 5.2 is ssentative of a typical hexagonal tube section that was analyzed for the second phase te research study with the LS-DYNA model LS1.8-1-HEX views being presented. hown, the hexagonal tube section post-buckling deformation lobes consist primarily he symmetric extensional Type II mode. The deformation plots for a typical gonal section are shown in Figure 5.3 with the views shown corresponding to the LS- 
DYNA model LS1.8-1-OCT. Similar to the hexagonal tube section shown in Figure 5.2, the octagonal tube section post-buckling deformation characteristics are primarily manifested by symmetric extensional Type II mode lobe deformations.

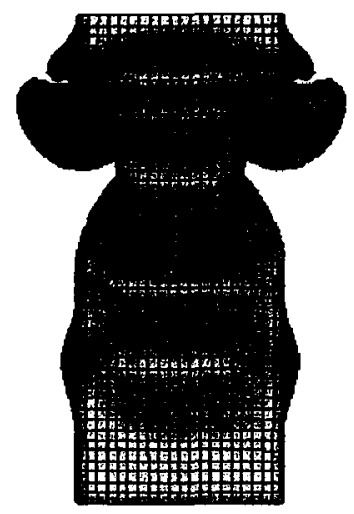

Front liew

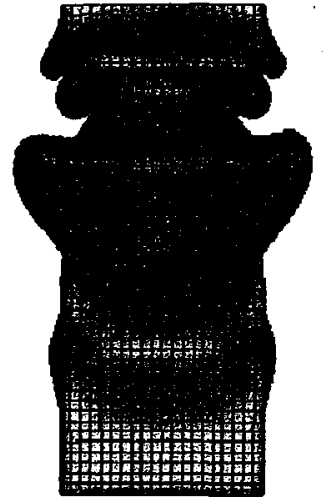

Side Vien:

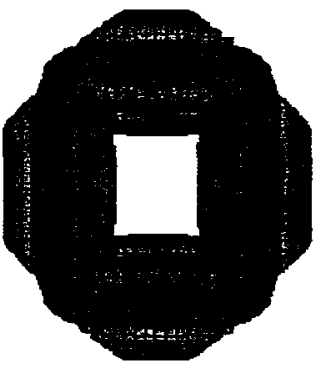

Top View:

Figure 5.1 - Final post-buckling deformation views of LS-DYNA model LS1.8-1-SQ.

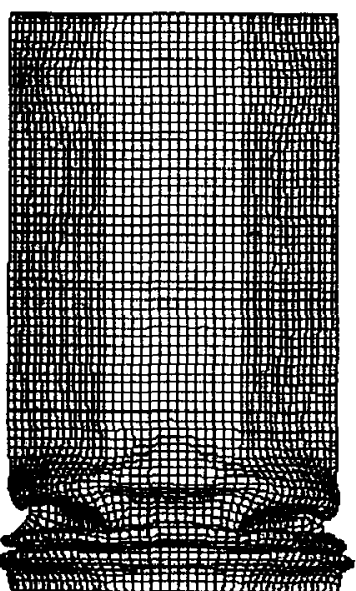

Front Vient:

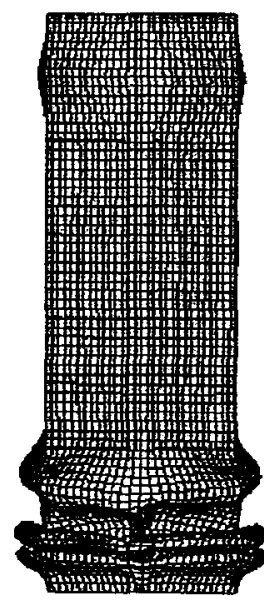

Side Vien.

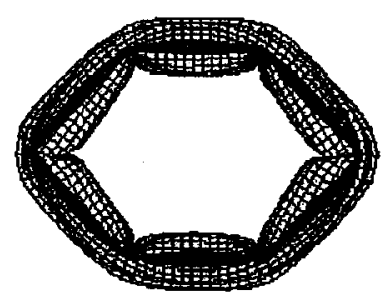

Top View

Figure 5.2 - Final post-buckling deformation views of LS-DYNA model LS1.8-1-HEX. 


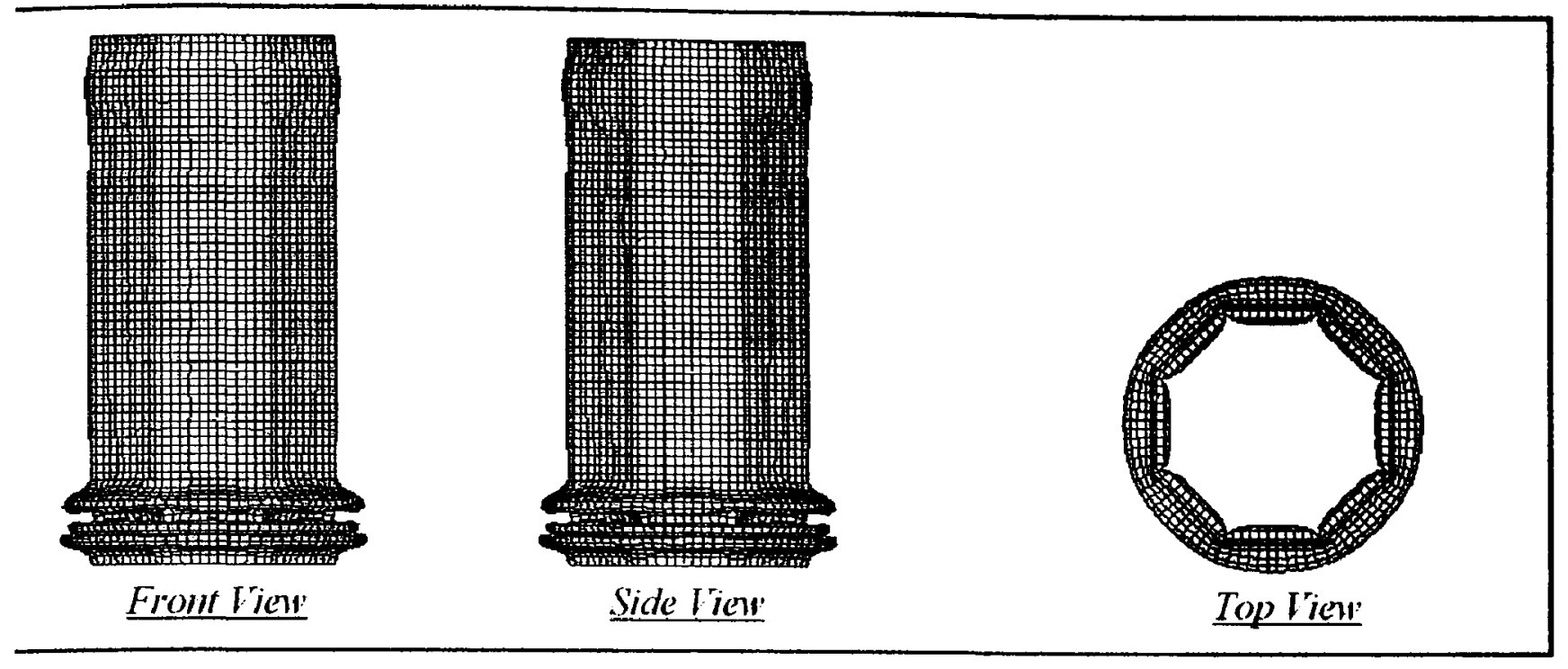

Figure 5.3 - Final post-buckling deformation views of LS-DYNA model LS1.8-1-OCT.

In association with the post-buckling deformation plots, the dynamic axial crushing force versus permanent displacement graph was presented for each representative tube section to highlight the progressive buckling phenomenon of the tube member. This graph illustrates the associated fluctuations in the dynamic axial crushing force in the form of the peak and troughs that are typical for an axially crushed member. The area under this graph is equivalent to the total energy absorbed by the axially crushed member. The energy absorption can be determined by integration of the force-deformation curve, and absorbed energy divided by the deformation defines the mean crush load for a given deformation. The mean crush load is the accepted primary response parameter to evaluate a column's capability of absorbing energy. The LSTC post-processing module within LS-DYNA provides the ability to output various results parameters (such as relative velocities, accelerations, kinetic energy, total energy, stresses, strains, etc.) pertaining to specific elements or, if desired, the global model. For instance, in the case of the mean axial crushing force, the relative kinetic energy values at a specific time smental (i.e., 0.0001 second) were directly output from the numerical computational Its in the form of text files that were further processed using EXCEL to obtain the ins featured in Figures 5.4 to 5.6. The graphs for the remaining specimens can be Id in the Appendix A section of this report. The relevant theory was presented in 
Section 2.2 Dynamic Progressive Buckling of this thesis that describes the formulation. Other items that are of interest are the crush force efficiency $\left(A_{E}\right)$ and inertia effects. In addition, the LS-DYNA numerical simulation results shall be compared to the theoretical closed form methods such as the Super Folding Element Method, Dynamic Amplification Factor Method, and Strain Rate Sensitivity Effect Method which were presented in Section 2.2 Dynamic Progressive Buckling of this thesis.

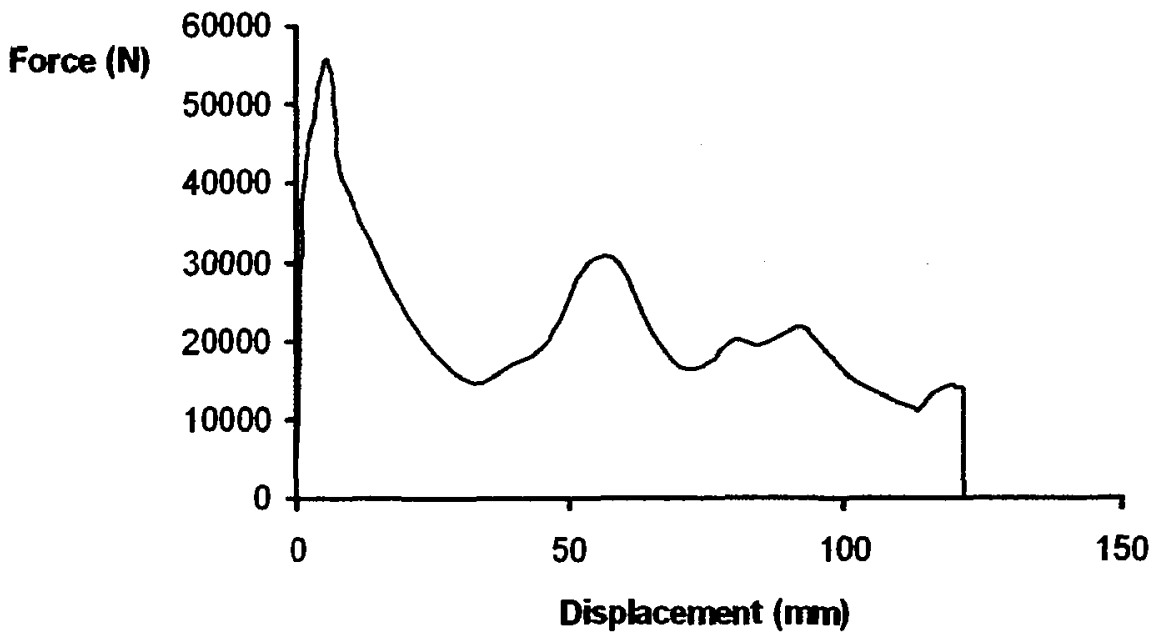

Figure 5.4 - Typical square tube section dynamic axial crushing force vs permanent displacement (Model LS1.8-1-SQ shown).

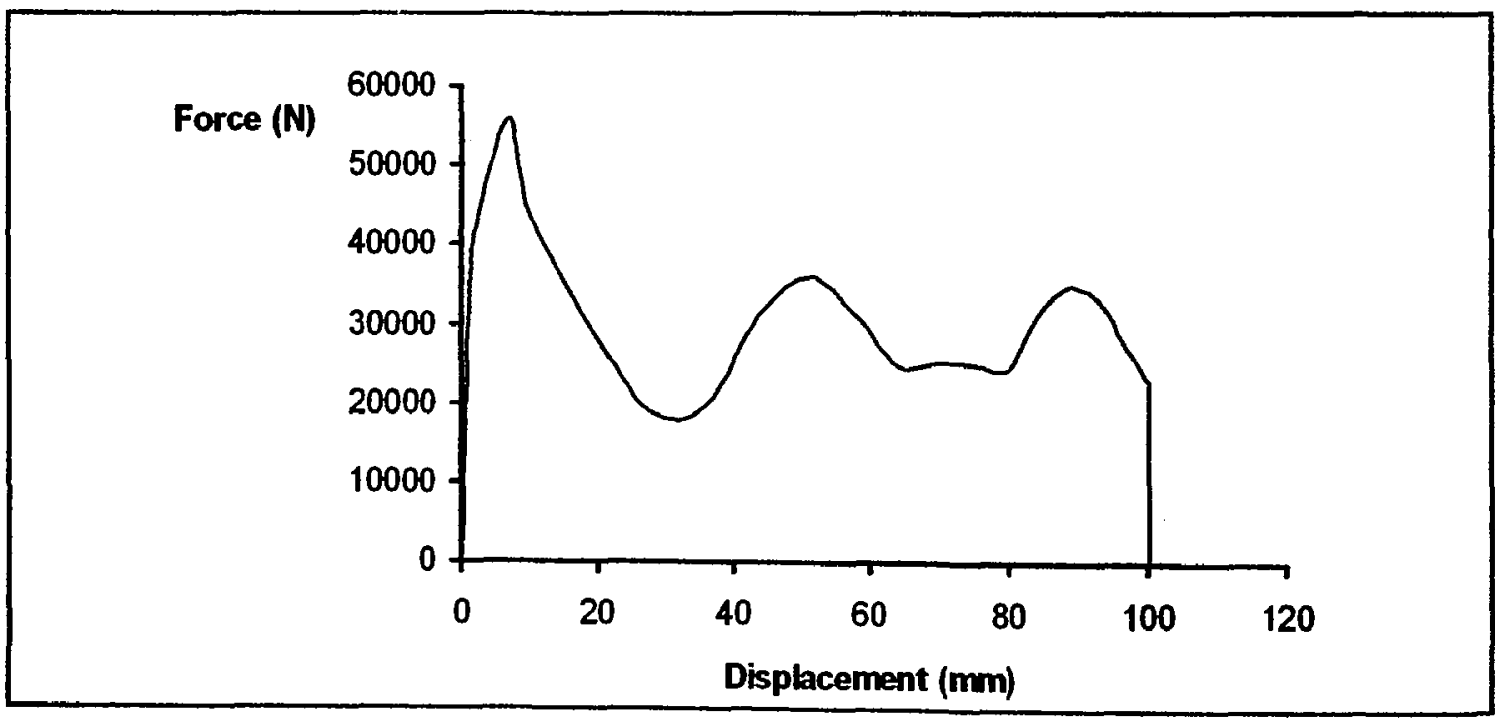

Figure 5.5 - Typical hexagonal tube section dynamic axial crushing force vs permanent displacement (Model LS1.8-1-HEX shown). 


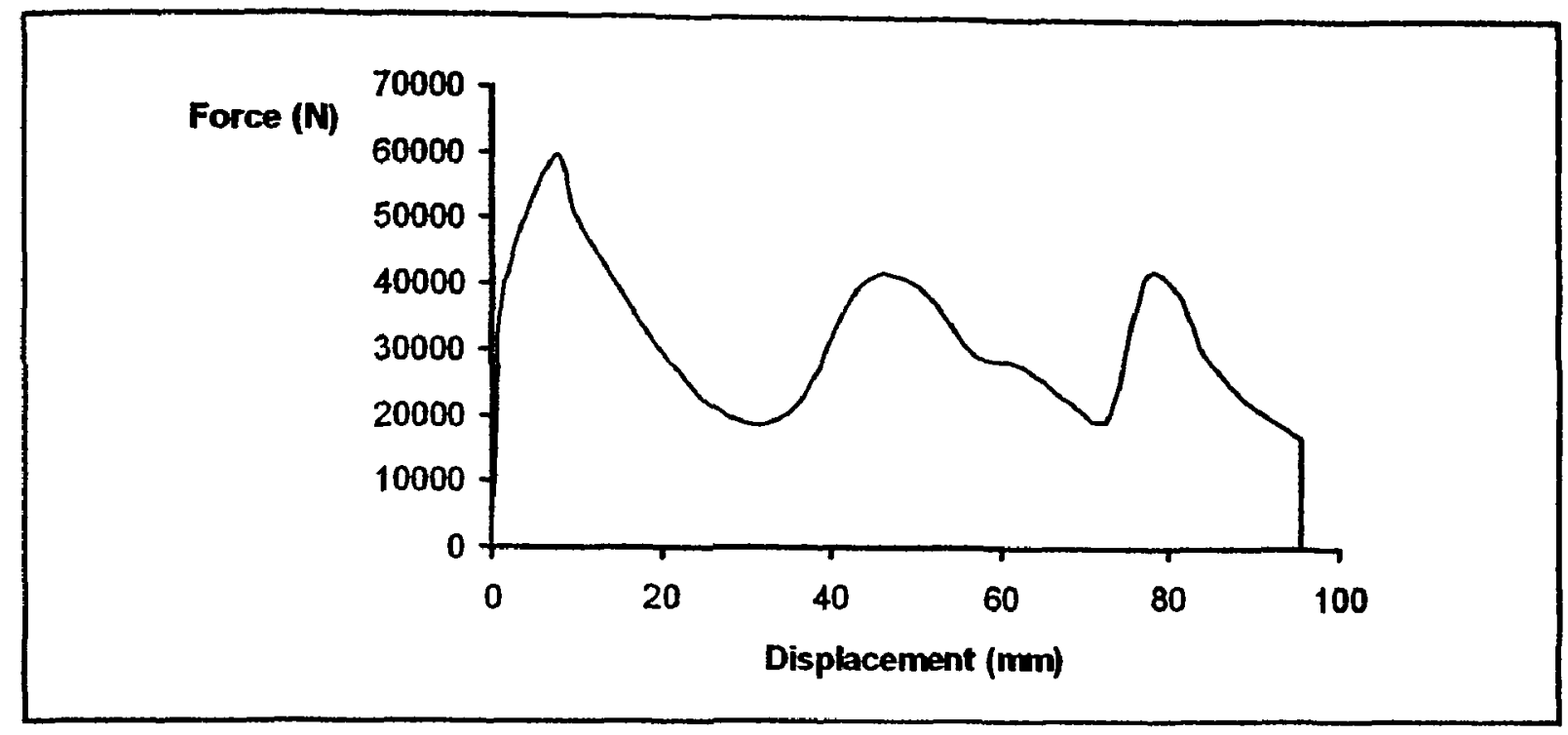

Figure 5.6-Typical octagonal tube section dynamic axial crushing force vs permanent displacement (Model LS1.8-1-OCT shown).

A second graph, the permanent displacement of the axially crushed member versus the duration of the impact event compliments the dynamic axial crushing force versus permanent displacement graph. The graph for each representative tube section is presented in Figure 5.7 to 5.9. This graph not only provides the time of the crushing effect, but it also helps to assess the effectiveness of the member in dissipating the impact energy. The non-linearity of the plotted data shows that the energy dissipation rate varies over the course of the impact event. The cause and significance of this shall be further highlighted in Section 5.2 of this thesis. 


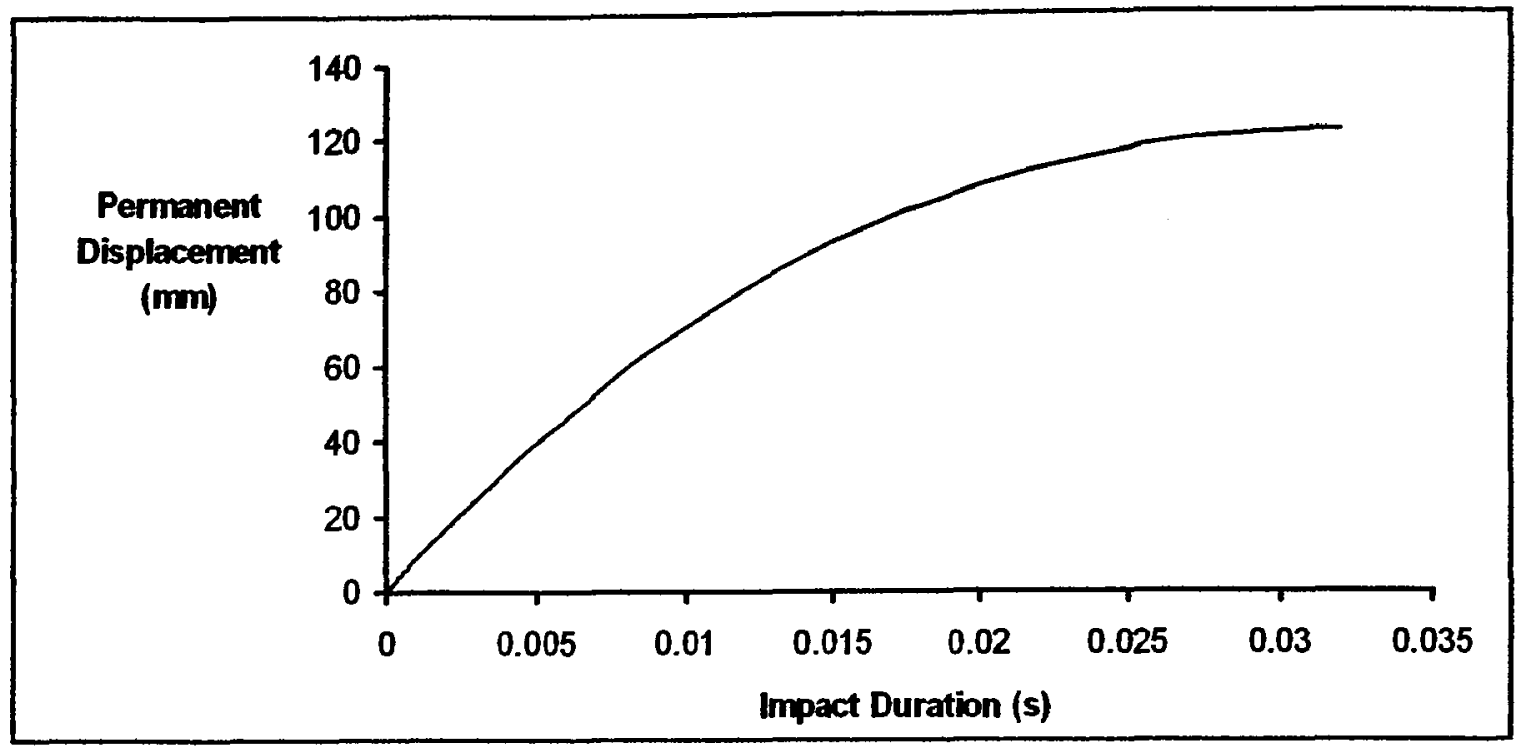

Figure 5.7 - Typical square tube section permanent displacement vs impact duration (Model LS1.8-1-SQ shown).

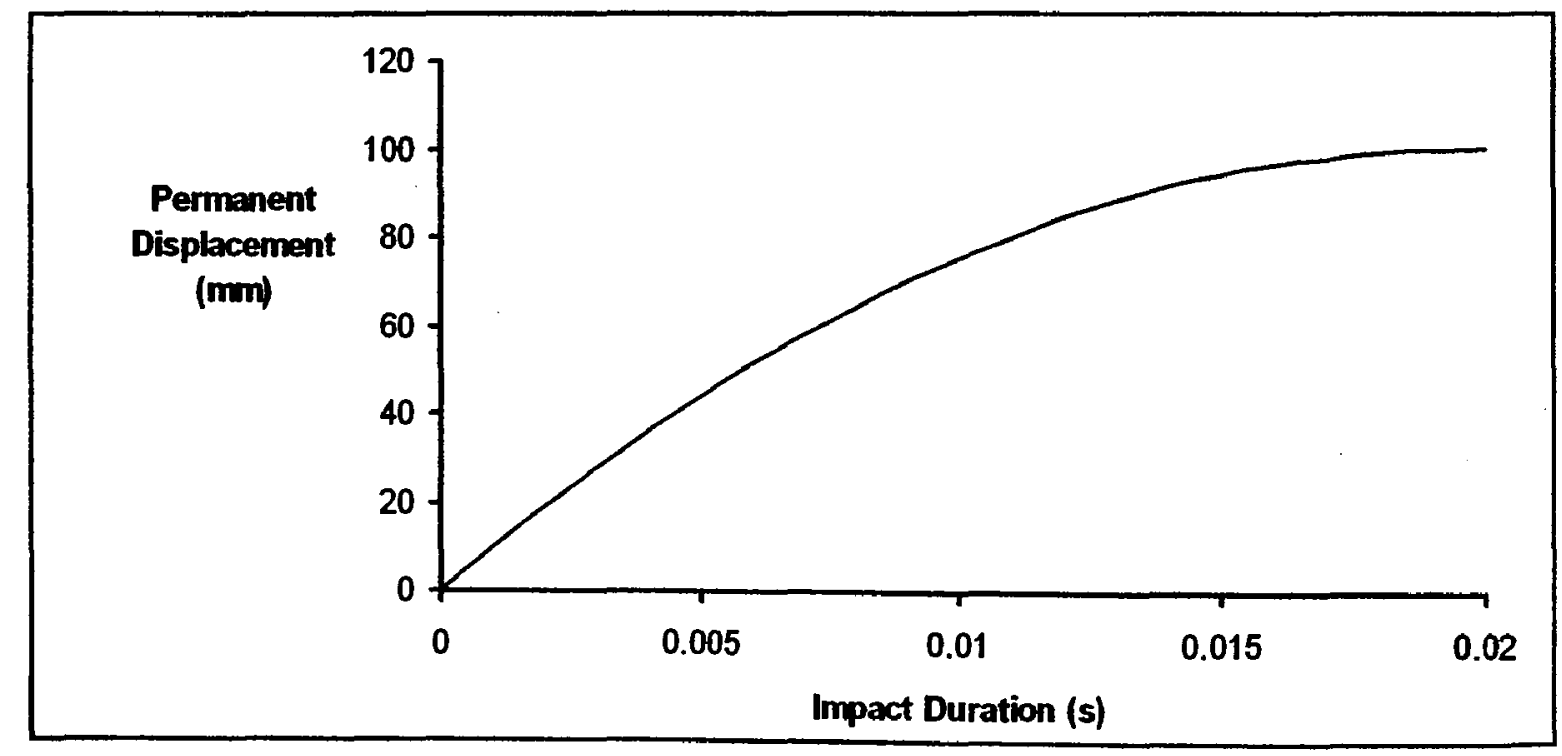

Figure 5.8 - Typical hexagonal tube section permanent displacement vs impact duration (Model LS1.8-1-HEX shown). 


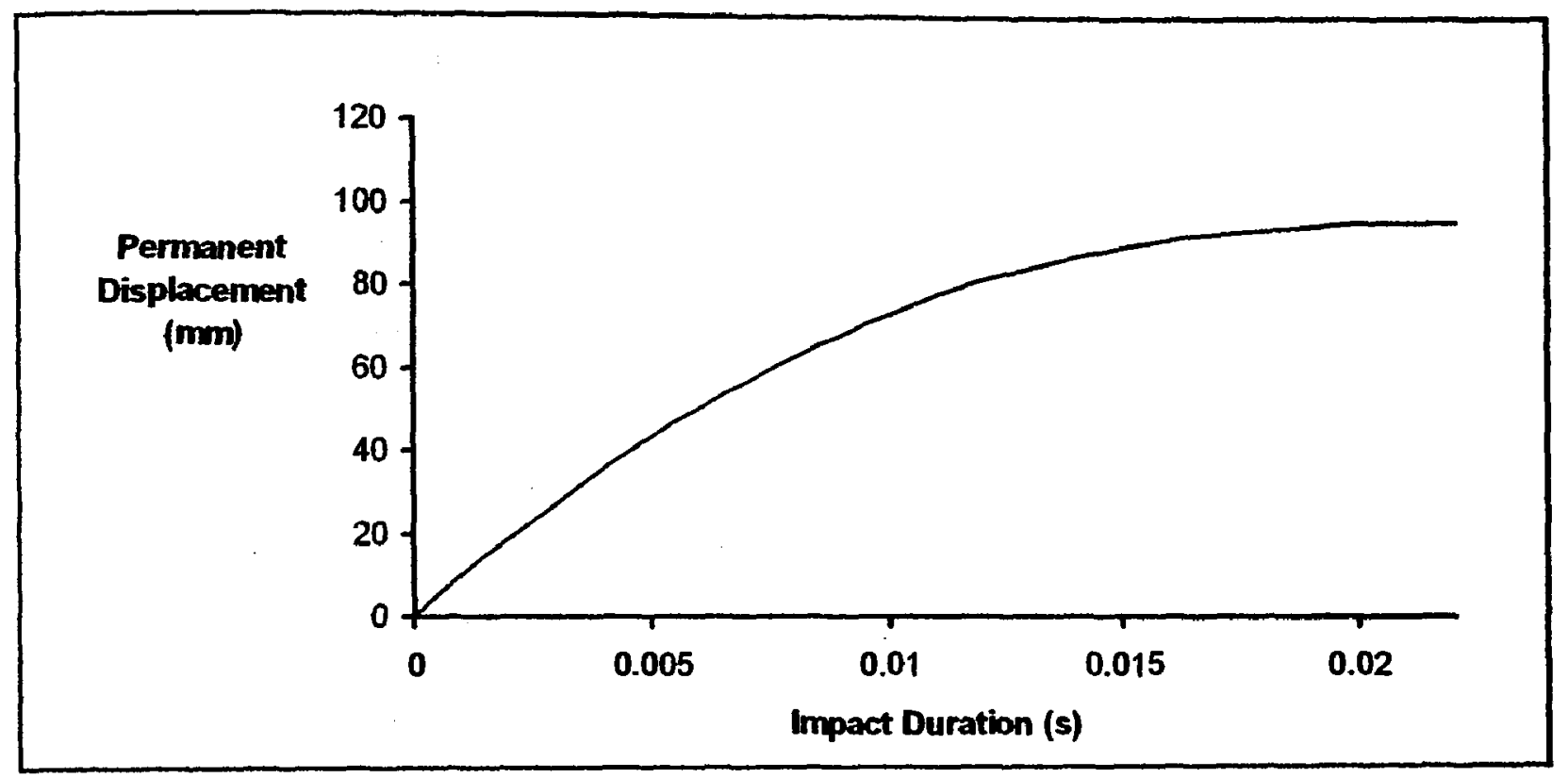

Figure 5.9 - Typical octagonal tube section permanent displacement vs impact duration (Model LS1.8-1-OCT shown). 
Table 5.I - LS-DYNA simulation results for square extrusion tube.

\begin{tabular}{|c|c|c|c|c|c|c|}
\hline $\begin{array}{c}\text { LS-DYNA } \\
\text { Model }\end{array}$ & $\begin{array}{c}\mathbf{t} \\
(\mathbf{m m})\end{array}$ & $\begin{array}{c}\mathbf{A} \\
\left(\mathbf{m m}^{\mathbf{2}}\right)\end{array}$ & $\begin{array}{c}\mathbf{V} \\
(\mathbf{m} / \mathbf{s})\end{array}$ & $\begin{array}{c}\boldsymbol{\delta}_{\mathbf{p}} \\
(\mathbf{m m})\end{array}$ & $\begin{array}{c}\mathbf{A}_{\mathbf{e}} \\
(\mathbf{\%})\end{array}$ & $\begin{array}{c}\mathbf{P}_{\mathbf{m d}} \\
(\mathbf{K N})\end{array}$ \\
\hline LS1.8-1-SQ & 1.80 & 581 & 10.28 & 122 & 39.4 & 24.0 \\
LS1.8-2-SQ & 1.80 & 581 & 10.17 & 130 & 41.9 & 21.9 \\
LS1.8-3-SQ & 1.80 & 581 & 8.47 & 85 & 27.4 & 23.2 \\
LS2.0-4-SQ & 2.00 & 625 & 11.64 & 128 & 41.3 & 29.3 \\
LS2.0-5-SQ & 2.00 & 625 & 14.06 & 191 & 61.6 & 28.5 \\
LS2.0-6-SQ & 2.00 & 625 & 9.45 & 81 & 26.1 & 30.4 \\
LS2.5-7-SQ & 2.50 & 773 & 13.20 & 121 & 39.0 & 39.7 \\
LS2.5-8-SQ & 2.50 & 773 & 11.10 & 75 & 24.2 & 45.3 \\
LS2.5-9-SQ & 2.50 & 773 & 15.60 & 160 & 51.6 & 42.0 \\
\hline
\end{tabular}

Table 5.2 - LS-DYNA simulation results for hexagonal extrusion tube.

\begin{tabular}{|c|c|c|c|c|c|c|}
\hline $\begin{array}{c}\text { LS-DYNA } \\
\text { Model }\end{array}$ & $\begin{array}{c}\mathbf{t} \\
(\mathbf{m m})\end{array}$ & $\begin{array}{c}\mathbf{A} \\
\left(\mathbf{m m}^{\mathbf{2}}\right)\end{array}$ & $\begin{array}{c}\mathbf{V} \\
(\mathbf{m} / \mathbf{s})\end{array}$ & $\begin{array}{c}\boldsymbol{\delta}_{\mathbf{p}} \\
(\mathbf{m m})\end{array}$ & $\begin{array}{c}\mathbf{A}_{\mathbf{e}} \\
(\mathbf{\%})\end{array}$ & $\begin{array}{c}\mathbf{P}_{\mathbf{m d}} \\
(\mathbf{K N})\end{array}$ \\
\hline LS1.8-1-HEX & 1.80 & 581 & 10.28 & 100 & 32.3 & 29.1 \\
LS1.8-2-HEX & 1.80 & 581 & 10.17 & 97 & 31.3 & 29.4 \\
LS1.8-3-HEX & 1.80 & 581 & 8.47 & 68 & 22.0 & 29.0 \\
LS2.0-4-HEX & 2.00 & 625 & 11.64 & 115 & 37.1 & 32.5 \\
LS2.0-5-HEX & 2.00 & 625 & 14.06 & 170 & 54.8 & 32.1 \\
LS2.0-6-HEX & 2.00 & 625 & 9.45 & 73 & 23.5 & 33.8 \\
LS2.5-7-HEX & 2.50 & 773 & 13.20 & 95 & 30.6 & 50.7 \\
LS2.5-8-HEX & 2.50 & 773 & 11.10 & 65 & 20.9 & 52.6 \\
LS2.5-9-HEX & 2.50 & 773 & 15.60 & 134 & 43.3 & 50.1 \\
\hline
\end{tabular}

Table 5.3 - LS-DYNA simulation results for octagonal extrusion tube.

\begin{tabular}{|c|c|c|c|c|c|c|}
\hline $\begin{array}{c}\text { LS-DYNA } \\
\text { Model }\end{array}$ & $\begin{array}{c}\mathbf{t} \\
(\mathbf{m m})\end{array}$ & $\begin{array}{c}\mathbf{A} \\
\left(\mathbf{m m}^{2}\right)\end{array}$ & $\begin{array}{c}\mathbf{V} \\
(\mathbf{m} / \mathbf{s})\end{array}$ & $\begin{array}{c}\boldsymbol{\delta}_{\mathbf{p}} \\
(\mathbf{m m})\end{array}$ & $\begin{array}{c}\mathbf{A}_{\mathbf{e}} \\
(\mathbf{\%})\end{array}$ & $\begin{array}{c}\mathbf{P}_{\mathbf{m d}} \\
(\mathbf{K N})\end{array}$ \\
\hline LS1.8-1-OCT & 1.80 & 581 & 10.28 & 96 & 30.8 & 30.5 \\
LS1.8-2-OCT & 1.80 & 581 & 10.17 & 90 & 29.1 & 31.6 \\
LS1.8-3-OCT & 1.80 & 581 & 8.47 & 61 & 19.5 & 32.7 \\
LS2.0-4-OCT & 2.00 & 625 & 11.64 & 104 & 33.6 & 35.8 \\
LS2.0-5-OCT & 2.00 & 625 & 14.06 & 147 & 47.5 & 37.0 \\
LS2.0-6-OCT & 2.00 & 625 & 9.45 & 65 & 21.0 & 37.9 \\
LS2.5-7-OCT & 2.50 & 773 & 13.20 & 86 & 27.9 & 55.6 \\
LS2.5-8-OCT & 2.50 & 773 & 11.10 & 58 & 18.8 & 58.3 \\
LS2.5-9-OCT & 2.50 & 773 & 15.60 & 122 & 39.3 & 55.1 \\
\hline
\end{tabular}


Table 5.4 - Mean axial crushing force (Pm) determined using the Super Folding Element Method.

\begin{tabular}{|c|c|c|c|c|}
\hline $\mathbf{N}$ & $\begin{array}{c}\mathbf{t} \\
(\mathbf{m m})\end{array}$ & $\begin{array}{c}\mathbf{A} \\
\left(\mathbf{m m}^{2}\right)\end{array}$ & $\begin{array}{c}\sigma_{\mathbf{o}} \\
\left(\mathbf{N} / \mathbf{m m}^{2}\right)\end{array}$ & $\begin{array}{c}\mathbf{P}_{\mathbf{m}} \\
(\mathbf{K N})\end{array}$ \\
\hline 4 & 1.8 & 581 & 107 & 11.0 \\
4 & 2.0 & 625 & 116.5 & 13.8 \\
4 & 2.5 & 773 & 123 & 20.3 \\
6 & 1.8 & 581 & 107 & 13.5 \\
6 & 2.0 & 625 & 116.5 & 16.9 \\
6 & 2.5 & 773 & 123 & 24.9 \\
8 & 1.8 & 581 & 107 & 15.6 \\
8 & 2.0 & 625 & 116.5 & 19.6 \\
8 & 2.5 & 773 & 123 & 28.7 \\
\hline
\end{tabular}

Table 5.5 - Dynamic mean axial crushing force $\left(\mathrm{P}_{\mathrm{m}, \mathrm{D} \mathrm{yn}}\right)$ of LS-DYNA models predicted using the Dynamic Amplification Method.

\begin{tabular}{|c|c|c|c|c|c|c|c|}
\hline $\begin{array}{c}\text { LS-DYNA } \\
\text { MODEL }\end{array}$ & $\begin{array}{c}\mathbf{t} \\
(\mathbf{m m})\end{array}$ & $\begin{array}{c}\mathbf{b} \\
(\mathbf{m m})\end{array}$ & $\begin{array}{c}\mathbf{V} \\
(\mathbf{m} / \mathbf{s})\end{array}$ & $\begin{array}{c}\sigma_{\mathbf{q}} \\
\left(\mathbf{N} / \mathbf{m m}^{\mathbf{2}}\right)\end{array}$ & $\mathbf{D}_{\text {amp }}$ & $\begin{array}{c}\mathbf{P}_{\mathbf{m}} \\
(\mathbf{K N})\end{array}$ & $\begin{array}{c}\mathbf{P}_{\mathbf{m}}, \mathbf{D y n} \\
(\mathbf{K N})\end{array}$ \\
\hline LS1.8-1-SQ & 1.8 & 80 & 10.28 & 107 & 1.25 & 11.0 & 13.8 \\
LS1.8-2-SQ & 1.8 & 80 & 10.17 & 107 & 1.25 & 11.0 & 13.8 \\
LS1.8-3-SQ & 1.8 & 80 & 8.47 & 107 & 1.21 & 11.0 & 13.3 \\
LS2.0-4-SQ & 2.0 & 80 & 11.64 & 116.5 & 1.26 & 13.8 & 17.4 \\
LS2.0-5-SQ & 2.0 & 80 & 14.06 & 116.5 & 1.32 & 13.8 & 18.2 \\
LS2.0-6-SQ & 2.0 & 80 & 9.45 & 116.5 & 1.21 & 13.8 & 16.8 \\
LS2.5-7-SQ & 2.5 & 80 & 13.2 & 123 & 1.26 & 20.3 & 25.5 \\
LS2.5-8-SQ & 2.5 & 80 & 11.1 & 123 & 1.22 & 20.3 & 24.7 \\
LS2.5-9-SQ & 2.5 & 80 & 15.6 & 123 & 1.30 & 20.3 & 26.5 \\
LS1.8-1-HEX & 1.8 & 53 & 10.28 & 107 & 1.21 & 13.5 & 16.3 \\
LS1.8-2-HEX & 1.8 & 53 & 10.17 & 107 & 1.20 & 13.5 & 16.2 \\
LS1.8-3-HEX & 1.8 & 53 & 8.47 & 107 & 1.17 & 13.5 & 15.8 \\
LS2.0-4-HEX & 2.0 & 53 & 11.64 & 116.5 & 1.21 & 16.9 & 20.5 \\
LS2.0-5-HEX & 2.0 & 53 & 14.06 & 116.5 & 1.25 & 16.9 & 21.3 \\
LS2.0-6-HEX & 2.0 & 53 & 9.45 & 116.5 & 1.17 & 16.9 & 19.8 \\
IS2 5-7-HEX & 2.5 & 53 & 13.2 & 123 & 1.21 & 24.9 & 30.0 \\
5-8-HEX & 2.5 & 53 & 11.1 & 123 & 1.17 & 24.9 & 29.2 \\
5-9-HEX & 2.5 & 53 & 15.6 & 123 & 1.24 & 24.9 & 31.0 \\
8-1-OCT & 1.8 & 40 & 10.28 & 107 & 1.18 & 15.6 & 18.4 \\
8-2-OCT & 1.8 & 40 & 10.17 & 107 & 1.18 & 15.6 & 18.3 \\
\hline
\end{tabular}


Table 5.5 (continued) - Dynamic mean axial crushing force $\left(\mathrm{P}_{\mathrm{m}, \mathrm{Dyn}}\right)$ determined using the Dynamic Amplification Method.

\begin{tabular}{|c|c|c|c|c|c|c|c|}
\hline $\begin{array}{c}\text { LS-DYNA } \\
\text { MODEL }\end{array}$ & $\begin{array}{c}\mathbf{t} \\
(\mathbf{m m})\end{array}$ & $\begin{array}{c}\mathbf{b} \\
(\mathbf{m m})\end{array}$ & $\begin{array}{c}\mathbf{V} \\
(\mathbf{m} / \mathbf{s})\end{array}$ & $\begin{array}{c}\mathbf{\sigma}_{\mathbf{o}} \\
\left(\mathbf{N} / \mathbf{m m}^{\mathbf{2}}\right)\end{array}$ & $\mathbf{D}_{\mathbf{a m p}}$ & $\begin{array}{c}\mathbf{P}_{\mathbf{m}} \\
(\mathbf{K N})\end{array}$ & $\begin{array}{c}\mathbf{P}_{\mathbf{m}, \mathbf{D}_{\text {Jns str }}} \\
(\mathbf{K N})\end{array}$ \\
\hline LS1.8-3-OCT & 1.8 & 40 & 8.47 & 107 & 1.15 & 15.6 & 17.9 \\
LS2.0-4-OCT & 2.0 & 40 & 11.64 & 116.5 & 1.18 & 19.6 & 23.1 \\
LS2.0-5-OCT & 2.0 & 40 & 14.06 & 116.5 & 1.22 & 19.6 & 23.9 \\
LS2.0-6-OCT & 2.0 & 40 & 9.45 & 116.5 & 1.15 & 19.6 & 22.5 \\
LS2.5-7-OCT & 2.5 & 40 & 13.2 & 123 & 1.18 & 28.7 & 33.8 \\
LS2.5-8-OCT & 2.5 & 40 & 11.1 & 123 & 1.15 & 28.7 & 33.0 \\
LS2.5-9-OCT & 2.5 & 40 & 15.6 & 123 & 1.21 & 28.7 & 34.8 \\
\hline
\end{tabular}

Table 5.6 - Dynamic mean axial crushing force $\left(\mathrm{P}_{\mathrm{m} \text { Dyn }}\right)$ of LS-DYNA models predicted using the Strain Rate Sensitivity Effect Method.

\begin{tabular}{|c|c|c|c|c|c|c|c|}
\hline $\begin{array}{c}\text { LS-DYNA } \\
\text { MODEL }\end{array}$ & $\begin{array}{c}\mathbf{t} \\
(\mathbf{m m})\end{array}$ & $\begin{array}{c}\mathbf{b} \\
(\mathbf{m m})\end{array}$ & $\begin{array}{c}\mathbf{V} \\
(\mathbf{m} / \mathbf{s})\end{array}$ & $\begin{array}{c}\boldsymbol{\sigma}_{\mathbf{q}} \\
\left(\mathbf{N} / \mathbf{m m}^{\mathbf{2}}\right)\end{array}$ & $\begin{array}{c}\text { Strain } \\
\text { Factor }\end{array}$ & $\begin{array}{c}\mathbf{P}_{\mathbf{m}} \\
(\mathbf{K N})\end{array}$ & $\begin{array}{c}\mathbf{P}_{\mathbf{m}, \mathbf{p y n}, \mathbf{s t r}} \\
(\mathbf{K N})\end{array}$ \\
\hline LS1.8-1-SQ & 1.8 & 80 & 10.28 & 107 & 1.28 & 11.0 & 14.2 \\
LS1.8-2-SQ & 1.8 & 80 & 10.17 & 107 & 1.28 & 11.0 & 14.2 \\
LS1.8-3-SQ & 1.8 & 80 & 8.47 & 107 & 1.27 & 11.0 & 14.0 \\
LS2.0-4-SQ & 2.0 & 80 & 11.64 & 116.5 & 1.29 & 13.8 & 17.9 \\
LS2.0-5-SQ & 2.0 & 80 & 14.06 & 116.5 & 1.31 & 13.8 & 18.1 \\
LS2.0-6-SQ & 2.0 & 80 & 9.45 & 116.5 & 1.28 & 13.8 & 17.7 \\
LS2.5-7-SQ & 2.5 & 80 & 13.2 & 123 & 1.30 & 20.3 & 26.4 \\
LS2.5-8-SQ & 2.5 & 80 & 11.1 & 123 & 1.29 & 20.3 & 26.2 \\
LS2.5-9-SQ & 2.5 & 80 & 15.6 & 123 & 1.32 & 20.3 & 26.7 \\
LS1.8-1-HEX & 1.8 & 53 & 10.28 & 107 & 1.32 & 13.5 & 17.8 \\
LS1.8-2-HEX & 1.8 & 53 & 10.17 & 107 & 1.31 & 13.5 & 17.7 \\
LS1.8-3-HEX & 1.8 & 53 & 8.47 & 107 & 1.30 & 13.5 & 17.6 \\
LS2.0-4-HEX & 2.0 & 53 & 11.64 & 116.5 & 1.32 & 16.9 & 22.4 \\
LS2.0-5-HEX & 2.0 & 53 & 14.06 & 116.5 & 1.34 & 16.9 & 22.7 \\
LS2.0-6-HEX & 2.0 & 53 & 9.45 & 116.5 & 1.31 & 16.9 & 22.2 \\
LS2.5-7-HEX & 2.5 & 53 & 13.2 & 123 & 1.34 & 24.9 & 33.2 \\
LS2.5-8-HEX & 2.5 & 53 & 11.1 & 123 & 1.32 & 24.9 & 32.9 \\
LS2.5-9-HEX & 2.5 & 53 & 15.6 & 123 & 1.35 & 24.9 & 33.6 \\
LS1.8-1-OCT & 1.8 & 40 & 10.28 & 107 & 1.34 & 15.6 & 20.9 \\
LS1.8-2-OCT & 1.8 & 40 & 10.17 & 107 & 1.34 & 15.6 & 20.8 \\
\hline
\end{tabular}


Table 5.6 (continued) - Dynamic mean axial crushing force $\left(\mathrm{P}_{\mathrm{m}, \mathrm{Dym} s \mathrm{st}}\right)$ determined using the Strain Rate Sensitivity Effect Method.

\begin{tabular}{|c|c|c|c|c|c|c|c|}
\hline $\begin{array}{c}\text { LS-DYNA } \\
\text { MODEL }\end{array}$ & $\begin{array}{c}\mathbf{t} \\
(\mathbf{m m})\end{array}$ & $\begin{array}{c}\mathbf{b} \\
(\mathbf{m m})\end{array}$ & $\begin{array}{c}\mathbf{V} \\
(\mathbf{m} / \mathbf{s})\end{array}$ & $\begin{array}{c}\sigma_{\mathbf{o}} \\
\left(\mathbf{N} / \mathbf{m m}^{2}\right)\end{array}$ & $\begin{array}{c}\text { Strain } \\
\text { Factor }\end{array}$ & $\begin{array}{c}\mathbf{P}_{\mathbf{m}} \\
(\mathbf{K N})\end{array}$ & $\begin{array}{c}\mathbf{P}_{\mathbf{m}, \text { Dymostr }} \\
(\mathbf{K N})\end{array}$ \\
\hline LS1.8-3-OCT & 1.8 & 40 & 8.47 & 107 & 1.32 & 15.6 & 20.6 \\
LS2.0-4-OCT & 2.0 & 40 & 11.64 & 116.5 & 1.35 & 19.6 & 26.4 \\
LS2.0-5-OCT & 2.0 & 40 & 14.06 & 116.5 & 1.37 & 19.6 & 26.7 \\
LS2.0-6-OCT & 2.0 & 40 & 9.45 & 116.5 & 1.33 & 19.6 & 26.0 \\
LS2.5-7-OCT & 2.5 & 40 & 13.2 & 123 & 1.36 & 28.7 & 39.0 \\
LS2.5-8-OCT & 2.5 & 40 & 11.1 & 123 & 1.34 & 28.7 & 38.6 \\
LS2.5-9-OCT & 2.5 & 40 & 15.6 & 123 & 1.38 & 28.7 & 39.5 \\
\hline
\end{tabular}


Table 5.7 - Table summarizing the dynamic mean axial crushing force determined by various methods (i.e. Experimental test, LS-DYNA, Dynamic Amplification Method, and Strain Rate Sensitivity Effect Method).

\begin{tabular}{|c|c|c|c|c|}
\hline \multicolumn{5}{|c|}{ Dynamic Mean Axial Crushing Force (KN) } \\
\hline $\begin{array}{l}\text { Tube } \\
\text { Section }\end{array}$ & $\begin{array}{c}\text { Experimental } \\
\text { Tests }\end{array}$ & $\begin{array}{c}\text { LS-DYNA } \\
\text { Simulations }\end{array}$ & $\begin{array}{c}\text { Dynamic } \\
\text { Amplification } \\
\text { Method }\end{array}$ & $\begin{array}{c}\text { Strain Rate } \\
\text { Sensitivity Effect } \\
\text { Method } \\
\end{array}$ \\
\hline 1.8-1-SQ & 24.6 & 24.0 & 13.8 & 14.2 \\
\hline 1.8-2-SQ & 21.8 & 21.9 & 13.8 & 14.2 \\
\hline 1.8-3-SQ & 22.5 & 23.2 & 13.3 & 14.0 \\
\hline 2.0-4-SQ & 29.3 & 29.3 & 17.4 & 17.9 \\
\hline $2.0-5-\mathrm{SQ}$ & 29.3 & 28.5 & 18.2 & 18.1 \\
\hline $2.0-6-\mathrm{SQ}$ & 29.7 & 30.4 & 16.8 & 17.7 \\
\hline 2.5-7-SQ & 40.6 & 39.7 & 25.5 & 26.4 \\
\hline 2.5-8-SQ & 46.0 & 45.3 & 24.7 & 26.2 \\
\hline 2.5-9-SQ & 41.9 & 42.0 & 26.5 & 26.7 \\
\hline 1.8-1-HEX & N/A & 29.1 & 16.3 & 17.8 \\
\hline 1.8-2-HEX & $\mathrm{N} / \mathrm{A}$ & 29.4 & 16.2 & 17.7 \\
\hline 1.8-3-HEX & N/A & 29.0 & 15.8 & 17.6 \\
\hline 2.0-4-HEX & N/A & 32.5 & 20.5 & 22.4 \\
\hline 2.0-5-HEX & N/A & 32.1 & 21.3 & 22.7 \\
\hline 2.0-6-HEX & N/A & 33.8 & 19.8 & 22.2 \\
\hline 2.5-7-HEX & N/A & 50.7 & 30.0 & 33.2 \\
\hline 2.5-8-HEX & N/A & 52.6 & 29.2 & 32.9 \\
\hline 2.5-9-HEX & N/A & 50.1 & 31.0 & 33.6 \\
\hline 1.8-1-OCT & N/A & 30.5 & 18.4 & 20.9 \\
\hline $1.8-2-0 C T$ & N/A & 31.6 & 18.3 & 20.8 \\
\hline 1.8-3-OCT & N/A & 32.7 & 17.9 & 20.6 \\
\hline 2.0-4-OCT & N/A & 35.8 & 23.1 & 26.4 \\
\hline $2.0-5-\mathrm{OCT}$ & N/A & 37.0 & 23.9 & 26.7 \\
\hline 2.0-6-OCT & N/A & 37.9 & 22.5 & 26.0 \\
\hline 2.5-7-OCT & N/A & 55.6 & 33.8 & 39.0 \\
\hline 2.5-8-OCT & N/A & 58.3 & 33.0 & 38.6 \\
\hline 2.5-9-OCT & N/A & 55.1 & 34.8 & 39.5 \\
\hline
\end{tabular}




\subsection{DISCUSSION}

Based on the numerical simulation results, it becomes apparent that the increase in the number of walls (flanges) has a direct impact on the mean axial crushing force $\left(\mathbf{P}_{\mathbf{m d}}\right)$ and permanent displacement $\left(\delta_{\mathrm{p}}\right)$ parameters. The mean axial crushing force $\left(\mathbf{P}_{\mathrm{mdd}}\right)$ and permanent displacement $\left(\delta_{\mathrm{p}}\right)$ parameters obtained from the numerical simulation results for both the hexagonal and octagonal tube sections were compared against the LS-DYNA baseline values of the square tube sections established in the first phase of the research study.

As presented below in Table 5.8, the adoption of a hexagonal tube section as an axially loaded energy absorbing column (based on the lower bound average value corresponding to the specimens with a nominal $2.0 \mathrm{~mm}$ wall thickness) would yield an average increase of $11 \%$ in the mean axial crushing force $\left(\mathrm{P}_{\mathrm{md}}\right)$ and an average decrease of $10 \%$ in the permanent displacement $\left(\delta_{\mathrm{p}}\right)$. The greatest benefits were demonstrated in the specimens with the thinnest nominal wall thickness $(1.8 \mathrm{~mm})$, where the upper bound average value yielded an average increase of $27 \%$ in the mean axial crushing force $\left(\mathrm{P}_{\mathrm{md}}\right)$ and an average decrease of $20 \%$ in the permanent displacement $\left(\delta_{p}\right)$.

Table 5.8 - Percentage difference of mean dynamic force $\left(\Delta \mathrm{P}_{\mathrm{md}}\right)$ and permanent axial displacement $\left(\Delta \delta_{p}\right)$ for hexagonal extrusion section compared to baseline square extrusion section LS-DYNA results.

\begin{tabular}{|c|c|c|c|c|}
\hline $\begin{array}{c}\text { LS-DYNA } \\
\text { Model }\end{array}$ & $\begin{array}{c}\boldsymbol{\delta}_{\mathbf{p}} \\
(\mathbf{m m})\end{array}$ & $\begin{array}{c}\Delta \boldsymbol{\delta}_{\mathbf{p}} \\
(\mathbf{\%})\end{array}$ & $\begin{array}{c}\mathbf{P}_{\mathbf{m d}} \\
(\mathbf{K N})\end{array}$ & $\begin{array}{c}\Delta \mathbf{P}_{\mathbf{m d}} \\
(\mathbf{\%})\end{array}$ \\
\hline LS1.8-1-HEX & 100 & -14 & 29.1 & 18 \\
LS1.8-2-HEX & 97 & -25 & 29.4 & 35 \\
LS1.8-3-HEX & 68 & -21 & 29.0 & 29 \\
\hline Average & & -20 & & 27 \\
\hline LS2.0-4-HEX & 115 & -9 & 32.5 & 11 \\
LS2.0-5-HEX & 170 & -9 & 32.1 & 10 \\
LS2.0-6-HEX & 73 & -11 & 33.8 & 14 \\
\hline Average & & -10 & & 11 \\
\hline LS2.5-7-HEX & 95 & -19 & 50.7 & 25 \\
LS2.5-8-HEX & 65 & -11 & 52.6 & 14 \\
LS2.5-9-HEX & 134 & -15 & 50.1 & 20 \\
\hline Average & & -15 & & 20 \\
\hline
\end{tabular}


Comparing the numerical simulation results obtained for the octagonal sectioned tubes to the LS-DYNA baseline values of the square tube sections revealed a similar trend to that established by the hexagonal section tube comparison to the baseline values. The magnitude of the mean axial crushing force $\left(\mathrm{P}_{\mathrm{md}}\right)$ and permanent displacement $\left(\delta_{\mathrm{p}}\right)$ parameters corresponding to the octagonal section tubes were higher than those achieved by their hexagonal counterparts. As presented in Table 5.9, the adoption of an octagonal tube section for an axially loaded energy absorbing column (based on the lower bound average values pertaining to the specimens with a nominal $2.0 \mathrm{~mm}$ wall thickness) would yield an average increase of $25 \%$ in the mean axial crushing force $\left(\mathbf{P}_{\mathrm{md}}\right)$ and an average decrease of $20 \%$ in the permanent displacement $\left(\delta_{p}\right)$. The greatest benefits were demonstrated in the specimens with the thinnest nominal wall thickness $(1.8 \mathrm{~mm})$, where the upper bound average values yielded an average increase of $38 \%$ in the mean axial crushing force $\left(\mathrm{P}_{\mathrm{md}}\right)$ and an average decrease of $26 \%$ in the permanent displacement $\left(\delta_{\mathrm{p}}\right)$.

Table 5.9 - Percentage difference of mean dynamic force $\left(\Delta \mathbf{P}_{\mathrm{md}}\right)$ and permanent axial displacement $\left(\Delta \delta_{p}\right)$ for octagonal extrusion section compared to baseline square extrusion section LS-DYNA results.

\begin{tabular}{|c|c|c|c|c|}
\hline $\begin{array}{c}\text { LS-DYNA } \\
\text { Model }\end{array}$ & $\begin{array}{c}\boldsymbol{\delta}_{\mathbf{p}} \\
(\mathbf{m m})\end{array}$ & $\begin{array}{c}\Delta \boldsymbol{\delta}_{\mathbf{p}} \\
\mathbf{( \% )}\end{array}$ & $\begin{array}{c}\mathbf{P}_{\mathbf{m d}} \\
(\mathbf{K N})\end{array}$ & $\begin{array}{c}\Delta \mathbf{P}_{\mathbf{m d}} \\
(\mathbf{\%})\end{array}$ \\
\hline LS1.8-1-OCT & 96 & -18 & 30.5 & 24 \\
LS1.8-2-OCT & 90 & -30 & 31.6 & 45 \\
LS1.8-3-OCT & 61 & -30 & 32.7 & 45 \\
\hline Average & & -26 & & 38 \\
\hline LS2.0-4-OCT & 104 & -18 & 35.8 & 22 \\
LS2.0-5-OCT & 147 & -20 & 37.0 & 26 \\
LS2.0-6-OCT & 65 & -21 & 37.9 & 27 \\
\hline Average & & -20 & & 25 \\
\hline LS2.5-7-OCT & 86 & -26 & 55.6 & 37 \\
LS2.5-8-OCT & 58 & -20 & 58.3 & 27 \\
LS2.5-9-OCT & 122 & -23 & 55.1 & 32 \\
\hline Average & & -23 & & 32 \\
\hline
\end{tabular}

A significant benefit in adopting one of the more complex section tubes (i.e., hexagon or octagon), aside from the increase in the mean axial crushing force, would be that the length of the given energy absorbing member can be reduced as a result of the lower 
permanent deformation. This reduction in the length of the undeformed member can provide additional weight savings. The stroke efficiency parameter, $A_{e}$, is a good indicator. Typically, the stroke efficiency parameter should be in the range of $80 \%$ to $95 \%$, and based on the numerical simulation results for the complex sections (see Table 5.2 and 5.3), they range from a lower bound value of $18.8 \%$ to a higher bound value of $54.8 \%$. Therefore, as a result of adopting either a hexagonal or octagonal tube section, there is a decrease in the stroke efficiency parameter over the benchmark square tube sections. In order to increase the $A_{e}$, the undeformed length of the tube should be decreased to fully maximize the benefits of the more complex section tubes.

The post buckling deformation characteristics exhibited by the computational analysis models pertaining to the hexagonal and octagonal tube sections consisted primarily of extensional (symmetric Type II) modes (see Section 2.2.1). Abromowicz and Jones $[11,12]$ demonstrated analytically that the difference in energy absorption between a symmetric and asymmetric mode (Type I) is indistinguishable between the two, but they found that extensional modes (Type II) had an increased energy absorption capacity. Thus, the extensional modes manifested by the LS-DYNA hexagonal and octagonal model deformations are the most efficient mechanism in dissipating impact energy in collapsing axial members. When plotting the graph depicting the dynamic axial crushing force $\left(P_{d}\right)$ vs the permanent displacement $\left(\delta_{p}\right)$ for the values based on the LS-DYNA results (see Figures 5.1 to 5.3), the generated curves exhibited similar characteristics to those of the experimental specimens. The typical curve shows that the tube initially behaves elastically with the load increasing at a steady rate, attaining an initial peak load followed by a rapid decrease in load.

Thereafter, the post-buckling phase is characterized by secondary peaks and troughs directly related to the lobe formations. The secondary peak loads are significantly lower agnitude compared to the initial peak load, but almost maintain a similar magnitude te progressive buckling of the tube continues. The large peak crushing force can be buted to inertia effects, as more energy is needed to initiate the folding process. The erical results data corresponding to the LS-DYNA model LS2.5-9-OCT (see 
Appendix A), which are typical of the computational analysis results, are used to illustrate the relevance of the lobe formation and its association with the dynamic axial crushing force $\left(P_{d}\right)$ vs the permanent displacement $\left(\delta_{p}\right)$ curve (see Figure 5.10).

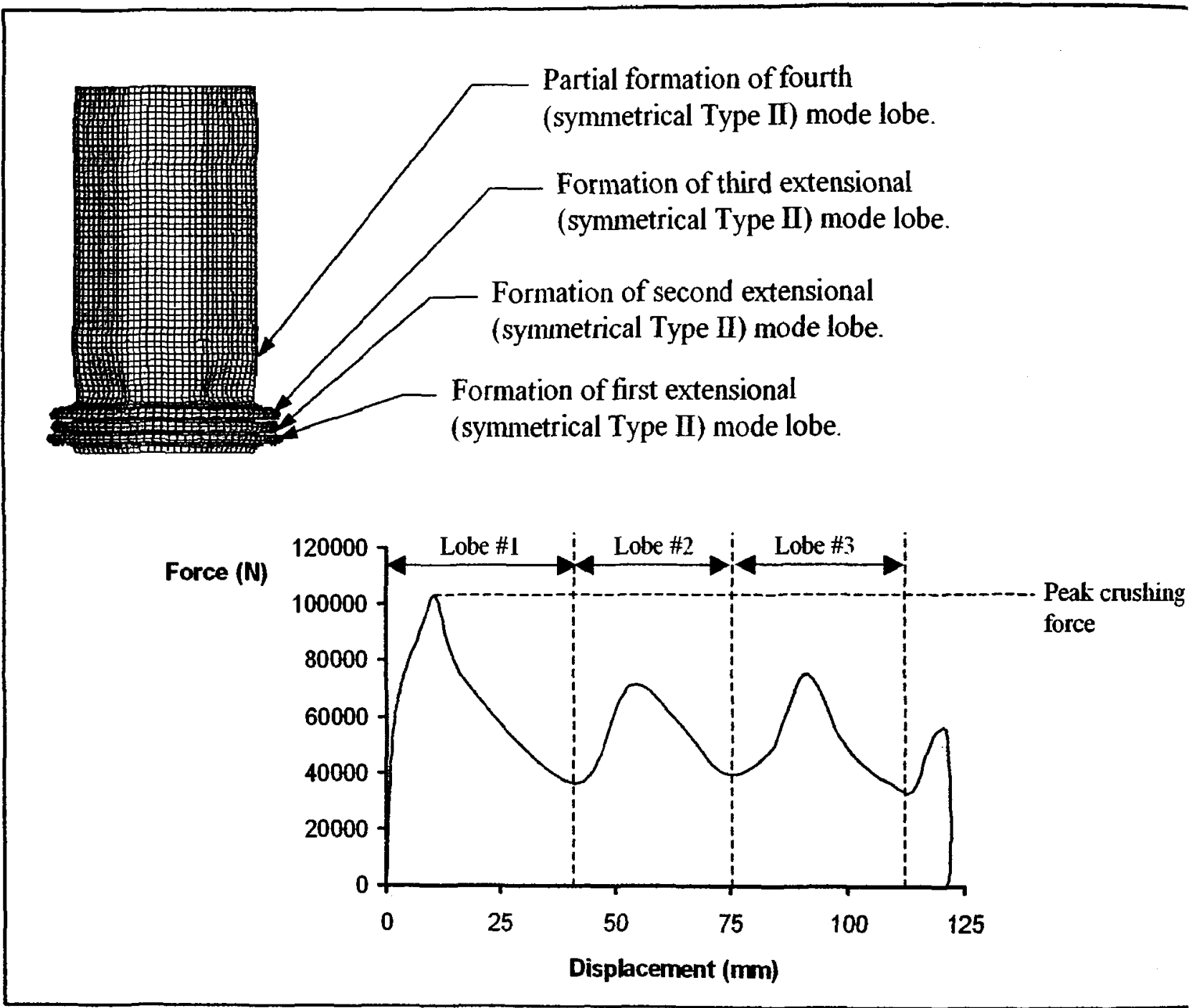

Figure 5.10 - Typical lobe formation relevance to the dynamic axial crushing force vs the permanent displacement curve.

Further review of the numerical simulation post-buckling deformation plots of the hexagonal and octagonal tube sections revealed an interesting insight into the nature of the post-buckling phenomenon. The crushing (folding) of the tube sections were primarily manifested by dynamic progressive buckling behaviour (see Section 2.2) as characterized by the lobe formations being confined primarily at one end. But, there also 
existed characteristics that are more closely associated with dynamic plastic buckling (see Section 2.3). It was noticed that a lateral deformation profile with a low mode number developed over the entire length of the tube in conjunction with the concentrated lobe deformations confined at one end of the tube. The lateral deformation was noticed at the impact end of the tube and perhaps the axial crushing effect of the tube is a combination of both the onset of a transient dynamic plastic buckling phase followed by the terminal dynamic progressive buckling phase. It was pointed out in Section 2.3 that dynamic plastic buckling produces a stress field within the shell during deformation that is biaxial, since both axial and radial deformations are evident, unlike dynamic progressive buckling, which is considered uniaxial and neglects the energy dissipation effects associated with radial deformations. This formation of a biaxial stress field is typically associated with inertia effects [10] and thus there exists a possibility that the peak crushing force discussed earlier is a direct result of dynamic plastic buckling, which is a very transient condition (short lived). Even Langseth and Hopperstad [5] observed that, in the beginning of an impact, inertia forces were set up as a result of the lateral movement of the sidewalls and thus were assumed to have a dominant effect. Their observations were based on limited experimental data that was not conducive to interpreting this effect, but they also did state that, as the displacement increases during the impact scenario, the dominance of the inertia effects disappears.

To further facilitate the understanding of the inertia effects and their relationship with dynamic plastic buckling, it was decided to plot the permanent displacement versus impact duration parameters. This permanent displacement versus impact duration graph provided both a qualitative and quantitative means to gauge the interactive effects between dynamic plastic buckling and dynamic progressive buckling. As shown in Figure 5.11, the slope of the curve has a steeper gradient for the phase of the deformation dominated by the preliminary phase of the impact event that corresponds to the inertia ts assumed to result from dynamic plastic buckling. The data in Figure 5.11 esponds to the LS-DYNA hexagonal model LS1.8-2-HEX, but is typically esentative of all the sections investigated (i.e., square, hexagonal, and octagonal). As onstrated in Figure 5.11, the formation of the first lobe which was identified as the 
peak crushing force, has a total permanent displacement of approximately $30 \mathrm{~mm}$ as deduced from the dynamic axial crushing force versus the permanent displacement curve. Translating the total permanent displacement value of $30 \mathrm{~mm}$, to the permanent displacement versus impact duration graph reveals that the time interval for this initial phase of the impact event is approximately 0.0035 seconds out of a total impact duration time of 0.022 seconds. In addition, the slope of the curve is at its greatest over this time interval.

In essence, the information derived from Figure 5.11 indicates that almost one-third of the total permanent displacement of the collapsed tube section occurred over a short time span equivalent to $16 \%$ of the whole impact duration. Based on these figures, it seems that there is a significant degree of impact energy dissipation occurring during this transient phase of the deformation that is highly characteristic of dynamic plastic buckling. Thus, it can be rationalized that the initial peak crushing force witnessed is a direct result of a transient condition that may in all likelihood be manifested by dynamic plastic buckling. Further experimental testing devoted to studying inertia effects would be beneficial to correlate the numerical simulation results with experimental data and establish some relationship. 


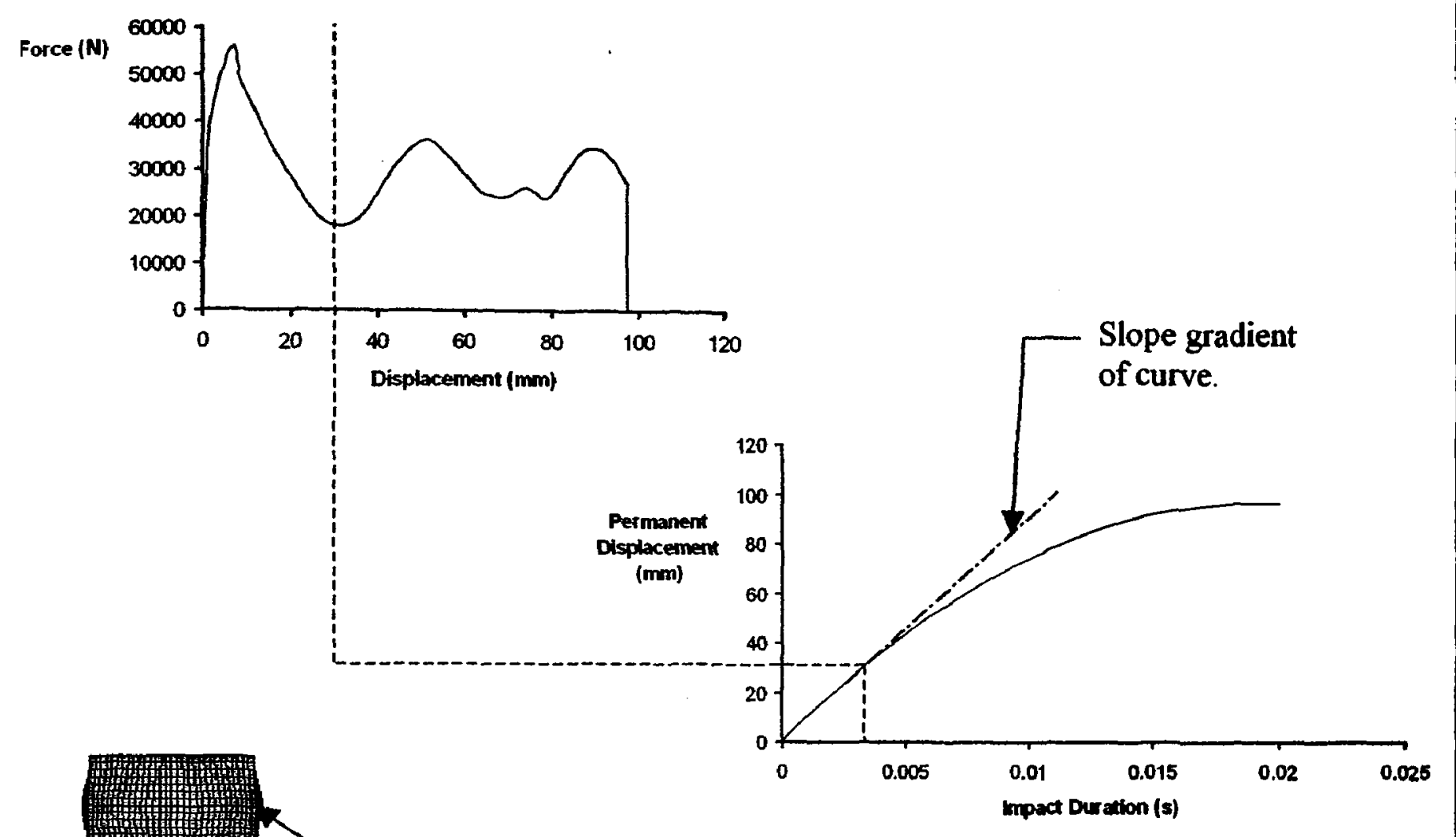

Formation of low mode lateral displacement that extends along entire length of tube.

ire 5.11 - Typical relationship showing dynamic plastic buckling effects using both the dynamic axial crushing force vs the permanent displacement curve and the permanent displacement vs impact duration curve. 


\subsubsection{REFINEMENT OF EXISTING ANALYTICAL METHODS}

Various methods such as the Dynamic Amplification Method (see Section 2.2.3.1) and the Strain Rate Sensitivity Effect Method (see Section 2.2.3.2), which are all variations of the Super Element Folding Method (see Section 2.2.2) proposed by Wierzbicki and Abramowicz [8], are overly conservative in their analytical prediction of the mean axial crushing force $\left(\mathbf{P}_{\mathrm{md}}\right)$. The Super Element Folding Method was based on several assumptions that simplify the complex interactions that occur during the axial crushing process, which, as a consequence, contribute directly to the conservativeness of this analytical approach. For instance, it was assumed that each lobe fold completely flattens the flanges after an axial displacement of $2 \mathrm{H}$ (see Figure 2.15), but as observed from the experimental test specimens and finite element results, that tends not to be the case. More importantly, the assumption of the flow stress $\left(\sigma_{0}\right)$ being constant, as assumed by Wierzbicki and Abramowicz [8,9,5], may give reasonable results for a material that is rigid, perfectly plastic and insensitive to strain rate. But in materials where significant strain hardening occurs after yielding, this over simplification for the stress flow may prove to be a source for inaccurate theoretical values. Such may be the case for the aluminium alloy based thin-walled extrusions used for this research study. As discussed previously, the actual deformation behaviour during dynamic loading is much more complicated owing to the fact that inertia forces have to be taken into account. It would be ideal for preliminary design and sizing purposes that the computational analysis results for the respective tube sections be integrated with the existing analytical methods.

This would allow the existing lower bound values pertaining to the dynamic mean axial crushing force $\left(P_{m d}\right)$ to be modified and provide less overly conservative (see Table 5.4 for lower bound $P_{m}$ values) analytical predictions. As presented in Table 5.10, the difference between the mean axial crushing force predicted using the Super Element Folding Method and computational numerical results is quite significant with a lower bound value of $82.7 \%$ (i.e., octagonal tube section with a nominal wall thickness of 2.5 $\mathrm{mm}$ ) and an upper bound value of $114.8 \%$ (i.e., hexagonal tube section with a nominal wall thickness of $1.8 \mathrm{~mm}$ ). Therefore, based on the trend defined from the finite element 
results, the values calculated using the Super Element Folding Method show that there is considerable room for improvement when it comes to yielding a more practical estimation for the dynamic mean axial crushing force $\left(P_{m d}\right)$. This leads one to incorporate a correction factor. The adopted correction factor would be based on the lower bound value of the $P_{m d}$ percentage increase (see Table 5.10) to preserve a degree of conservatism and at the same time improve the correlation between the values derived from the analytical and computational methods

Table 5.10 - Table showing comparison between mean axial crushing force predicted using the Super Element Folding Method and LS-DYNA numerical simulations.

\begin{tabular}{|c|c|c|c|c|c|c|}
\hline $\begin{array}{c}\text { Section } \\
\text { Tube }\end{array}$ & $\mathbf{N}$ & $\begin{array}{c}\mathbf{t} \\
(\mathbf{m m})\end{array}$ & $\begin{array}{c}\mathbf{A} \\
\left(\mathbf{m m}^{\mathbf{2}}\right)\end{array}$ & $\begin{array}{c}\mathbf{P}_{\mathbf{m}} \mathbf{} \\
(\mathbf{K N})\end{array}$ & $\begin{array}{c}\text { Minimum } \\
\mathbf{L S}-\mathbf{D Y N A} \\
\mathbf{P}_{\mathbf{m d}}(\mathbf{K N})\end{array}$ & $\begin{array}{c}\mathbf{P}_{\mathbf{m d}} \text { Increase } \\
\mathbf{( \% )}\end{array}$ \\
\hline Square & $\mathbf{4}$ & 1.8 & 581 & 11 & 21.9 & 99.1 \\
Square & 4 & 2 & $\mathbf{6 2 5}$ & 13.8 & 28.5 & 106.5 \\
Square & $\mathbf{4}$ & 2.5 & 773 & 20.3 & 39.7 & 95.6 \\
Hexagonal & $\mathbf{6}$ & 1.8 & 581 & 13.5 & 29 & 114.8 \\
Hexagonal & 6 & 2 & $\mathbf{6 2 5}$ & 16.9 & 32.1 & $\mathbf{8 9 . 9}$ \\
Hexagonal & $\mathbf{6}$ & 2.5 & 773 & 24.9 & 50.1 & 101.2 \\
Octagonal & $\mathbf{8}$ & 1.8 & 581 & 15.6 & 30.5 & 95.5 \\
Octagonal & $\mathbf{8}$ & 2 & $\mathbf{6 2 5}$ & 19.6 & 35.8 & $\mathbf{8 2 . 7}$ \\
Octagonal & $\mathbf{8}$ & 2.5 & $\mathbf{7 7 3}$ & $\mathbf{2 8 . 7}$ & 55.1 & $\mathbf{9 2 . 0}$ \\
\hline
\end{tabular}

** See Table 5.4

Therefore, a revised form of the Super Element Folding Method expression defined in Equation (2.29) might include the introduction of a correction factor of 1.80 which would be applicable to either a square, hexagonal, or octagonal tube section column. Hence, Equation (2.29) becomes:

$$
P_{m}=1.80\left[\frac{2}{3} \sigma_{0} t \sqrt{\pi N A}\right]
$$

or,

$$
P_{m}=1.2 \sigma_{0} t \sqrt{\pi N A}
$$


For improved estimates, the lower bound values of each specific tube section can be used to develop a more refined Super Element Folding Method expression. For the square and hexagonal tube sections, a correction factor of 1.95 and 1.89 , respectively, can be adopted based on the values presented in table 5.10. The correction factor of 1.80 developed previously would be applicable to only the octagonal sections. Therefore, the modified Super Element Folding Method expressions would be as follows

Square Section: $\quad P_{m}=1.30 \sigma_{0} t \sqrt{\pi N A}$

Hexagonal Section: $\quad P_{m}=1.26 \sigma_{0} t \sqrt{\pi N A}$

Octagonal Section: $\quad P_{m}=1.20 \sigma_{0} t \sqrt{\pi N A}$

Another note of interest is that the correction factor seems to decrease as the number of tube section flanges is increased. To further explore this relation, it would be prudent to conduct additional experimental and computational analysis in order to establish any validity to this trend

The above modified expressions defined by Equations (5.2) to (5.4) can be conservatively applied to any of the three different types of tube sections, serving as a practical design aid that significantly facilitates the preliminary selection and sizing of structural elements for crashworthiness applications. As illustrated by the formulation of Equation (5.1) with the application of the LS-DYNA numerical simulation results, parametric design curves that would prove very beneficial in crashworthiness design applications involving energy absorbing members can be easily adopted. 


\section{Chapter 6}

\section{CONCLUSION}

In summary, the computation simulations provided valuable insight into the complex axial deformation behaviour of the square tube members, revealing good correlation with the experimental test data for the first phase of the research study by predicting both the mean dynamic axial crushing force and permanent displacement within $\pm 5 \%$ of the experimental values. The good correlation between the experimental and numerical simulation results instilled confidence in the material parameter models that were developed for the finite element analysis and their application in validating more complex polygonal tube sections for the second phase of the research study. The second phase of the research study revealed that the optimization of a given tube section, by adopting a more complex polygonal section in the form of a hexagon or octagon, can provide significant increases in the energy dissipation capability of a column member. It was demonstrated from the LS-DYNA numerical simulation results that the hexagonal section tube had a lower bound average increase in the mean axial crushing force and permanent displacement parameters of $10 \%$ and $11 \%$, respectively, when compared to the baseline square tube sections from the first part of the research study. The octagonal tube section demonstrated even greater improvements over the baseline square tube sections. ysis yielded a lower bound average increase in the mean axial crushing force and ranent displacement parameters of $25 \%$ and $20 \%$, respectively. Similarly, the LSJA finite element numerical analyses have shown that the post-buckling deformation acteristics resulting from the dynamic axial impacts can also be predicted with a 
reasonable degree of accuracy. Such is the case for the first phase of the investigative study where the post-buckling deformation characteristics pertaining to the experimental test specimens and the numerical simulations showed good correlation. The distinct lobe formations comprising of symmetric Type I and Type II modes along with the asymmetric mixed mode lobe formations were accurately predicted for the various specimens. Furthermore, even the small amplitude buckles that extended over the entire length of the low impact velocitiy specimens and observed by both Langseth and Hopperstad [5] were predicted by the computational analysis.

The existing analytical procedure, Super Element Folding Method, utilized to estimate the mean dynamic axial crushing force of a column member has been shown to provide overly conservative values. Using the numerical simulation results as a basis, it was determined that the mean dynamic axial crushing force predicted using the Super Element Folding Method, can easily be improved by introducing a correction factor. Depending on the section geometry, the correction factor varies between of 1.8 (octagonal) to 1.95 (square). The application of a modified analytical method would greatly facilitate the sizing of energy absorbing members during the preliminary and conceptual design phases since the estimated values would be more representative of the actual values. As a result of adopting more complex polygonal section tubes for axial impact applications, the energy absorbing members can be made significantly shorter and as a result the corresponding weight will also be reduced when compared to more conventional square and rectangular configurations.

Related research work that can be inspired by this study may include the experimental validation of the crashworthiness behaviour of the hexagonal and octagonal tube sections predicted by computational analysis. Another interesting topic would involve the crashworthiness behaviour of tubes fabricated from composite materials instead of metal with an emphasis on fibre orientation for optimal performance and failure modes associated with the post-buckling deformation. Also, the role inertia plays in the preliminary phases of the impact can be studied in greater depth since this plays a pivotal role in the overall post-buckling deformation characteristics. 
And on a final note, the integration of numerical simulation codes as a tool in formulating parametric design relations and curves should be further investigated since it could facilitate the preliminary conceptual design phase by more accurately predicting the mean axial crushing force of a member and also because it is a less expensive alternative to experimental testing. 


\section{REFERENCES}

[1] Johnson, W., Mamalis, A.G., and Reid, S.R., Aspects of Car Design and Human Injury, Human Body Dynamics, Impact, Occupational and Athletic Aspects, Clarendon Oxford, pg. 181-200, 1982.

[2] Furrer, P. and Ruckstuhl, B., Lighting the Way For Bodies, Journal of Automotive Engineering, August 2003, pg. 38-44.

[3] Snyder, R.G., Human Impact Tolerance, SAE Paper 700398, International Automobile Safety Conference Compendium, SAE, pg. 712-82, 1970.

[4] Automotive Aluminium Crash Energy Management Manual, The Aluminium Association, Publication AT5, December 1998.

[5] Langseth, M.; Hopperstad, O.; Static and Dynamic Axial Crushing of Square Thin-Walled Aluminium Extrusions, International Journal of Impact Engineering 1996, 18(7-8): pg. 949-968.

[6] Langseth, M.; Hopperstad, O.; Berstad, T.; Crashworthiness of Aluminium Extrustions: Validation of Numerical Simulation, Effect of Mass Ratio and Impact Velocity, International Journal of Impact Engineering 1999, 22: pg. 829-854.

[7] Langseth, M.; Hopperstad, O.; Reyes, A.; Square Aluminium Tubes Subjected To Oblique Loading, International Journal of Impact Engineering 2003, 28: pg. 10771106.

[8] Abramowicz, W. and Wierzbicki, T.; On The Crushing Mechanics of Thin-Walled Structures, Journal of Applied Mechanics 1983, 50: pg. 727-739.

[9] Abramowicz, W. and Wierzbicki, T.; Axial Crushing of Multi-corner Sheet Metal Columns, Journal of Applied Mechanics 1989, 56: pg. 113-120.

[10] Jones, N.; Structural Impact, Cambridge University Press, 1989.

[11] Abramowicz, W. and Jones, N.; Dynamic Axial Crushing of Square Tubes, International Journal of Impact Engineering 1984, 2: pg. 179-208.

[12] Abramowicz, W. and Jones, N.; Dynamic Progressive Buckling of Circular and Square Tubes, International Journal of Impact Engineering 1986, 4: pg. 234-273.

[13] Aspden, R.J. and Campbell J.D., The Effect of Loading Rate on the Elasto-Plastic Flexure of Steel Beams, Proceedings of the Royal Society of London, Series A 1966, 290, pg. 266-285. 
[14] Marsh, K.J. and Campbell J.D., The Effect of Strain Rate on the Post-Yield Flow of Mild Steel, Journal of Mechanics and Physics of Solids, 11, pg. 49-63, 1963.

[15] Nicholas, T., Tensile Testing of Materials at High Rates of Strain, Experimental Mechanics, 21,pg. 177-185, 1981.

[16] Kolsky, H.; An Investigation of the Mechanical Properties of Materials at Very High Rates of Strain, Proc. Roy. Phys. Soc., B 62, pp. 676-700 (1949)

[17] Kaiser, M.; Advancements in the Spkit Hopkinson Bar Test-Masters Thesis, Virginia Polytechnic State; 1998.

[18] Maiden, C.J. and Green, S.J., Compressive Strain-Rate Tests on Six Selected Materials at Strain Rates from $10^{-3}$ to $10^{4} \mathrm{in} / \mathrm{in} / \mathrm{sec}$, Journal of Applied Mechanics 1966, 33, pg. 496-504.

[19] Johnson, W. and Mellor, P., Engineering Plasticity, Von Nostrand Reinhold, London, 1973.

[20] Macaulay, M.A., Introduction to Impact Engineering, Chapman \& Hall, London, 1987.

[21] Karagiozova, D.; Dynamic Buckling of Elastic-Plastic Square Tubes Under Axial Impact - I: Stress Wave Propagation Phenomenon, International Journal of Impact Engineering 2004, 30: pg. 143-166.

[22] Kim, H.; New Extruded Multi-Cell Aluminium Profile For Maximum Crash Energy Absorption and Weight Efficiency, Thin Walled Structures 40 2002, pg. 311-327.

[23] Florence, A. and Goodier, J.; Dynamic Plastic Buckling of Cylindrical Shells in Sustained Axial Compressive Flow, Journal of Applied Mechanics, 35(1) 1968, pg. 80-86.

[24] Florence, A and Lindberg, H., Dynamic Pulse Buckling: Theory and Experiment, SRI International Report to Defence Nuclear Agency, February 1982.

[25] Langseth, M.; Hopperstad, O.; Hanssen, A; Static Crushing of Square Aluminium Extrusions With Aluminium Foam Filler, International Journal of Mechanical Sciences 1999, 41(7-8): pg. 967-993.

Opheim, B.; Bending of Thin-Walled Extrusions' Doctoral Thesis, Trondheim: Norwegian University of Science and Technology; 1996.

Hallquist, J, LS-DYNA 3D:Theoretical Mamual, Livermore Software Technology Corporation, 1993. 
[28] Thornton, P.H., Mahmood, H.F., and Magee, C.L., Energy Absorption By Structural Collapse, Structural Crashworthiness, Butterworths, London, pg. 96117, 1983. 


\section{PPENDIX A}

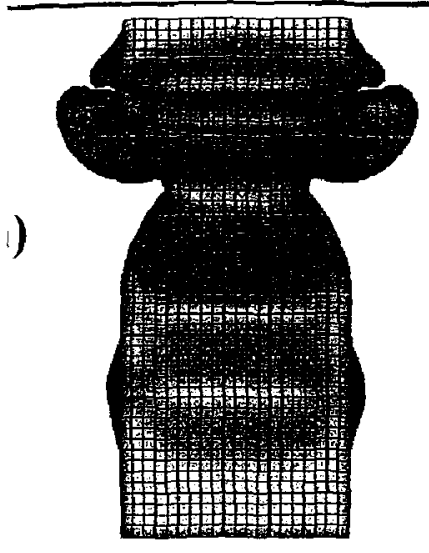

Front Fien:

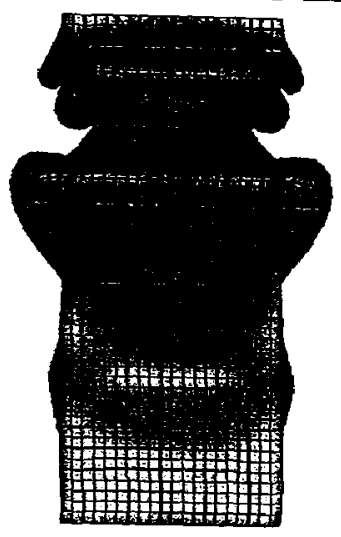

Side rien:

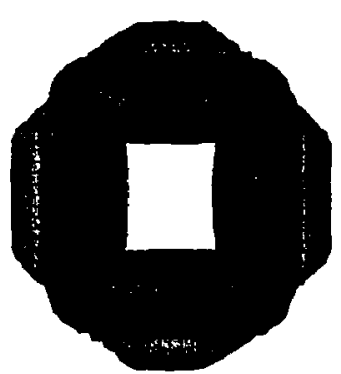

Top rien:

b)
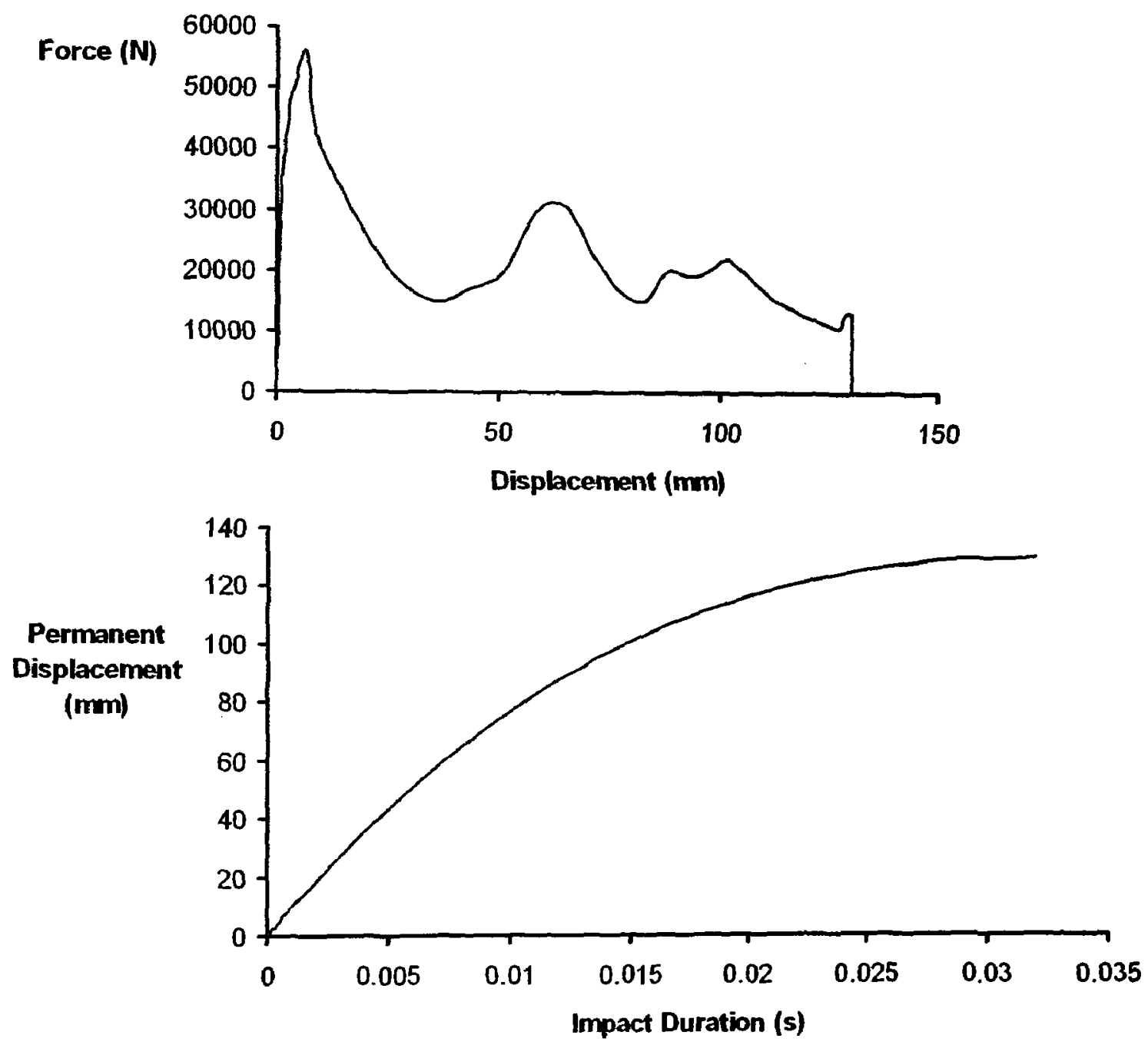

e A-1 (a) Final deformation views of LS-DYNA model LS1 8-2-SQ.

(b) LS 1.8-2- SQ Dynamic Axial Crushing Force vs Permanent Displacement.

(c) LS1.8-2- SQ Permanent Displacement vs Impact Duration. 


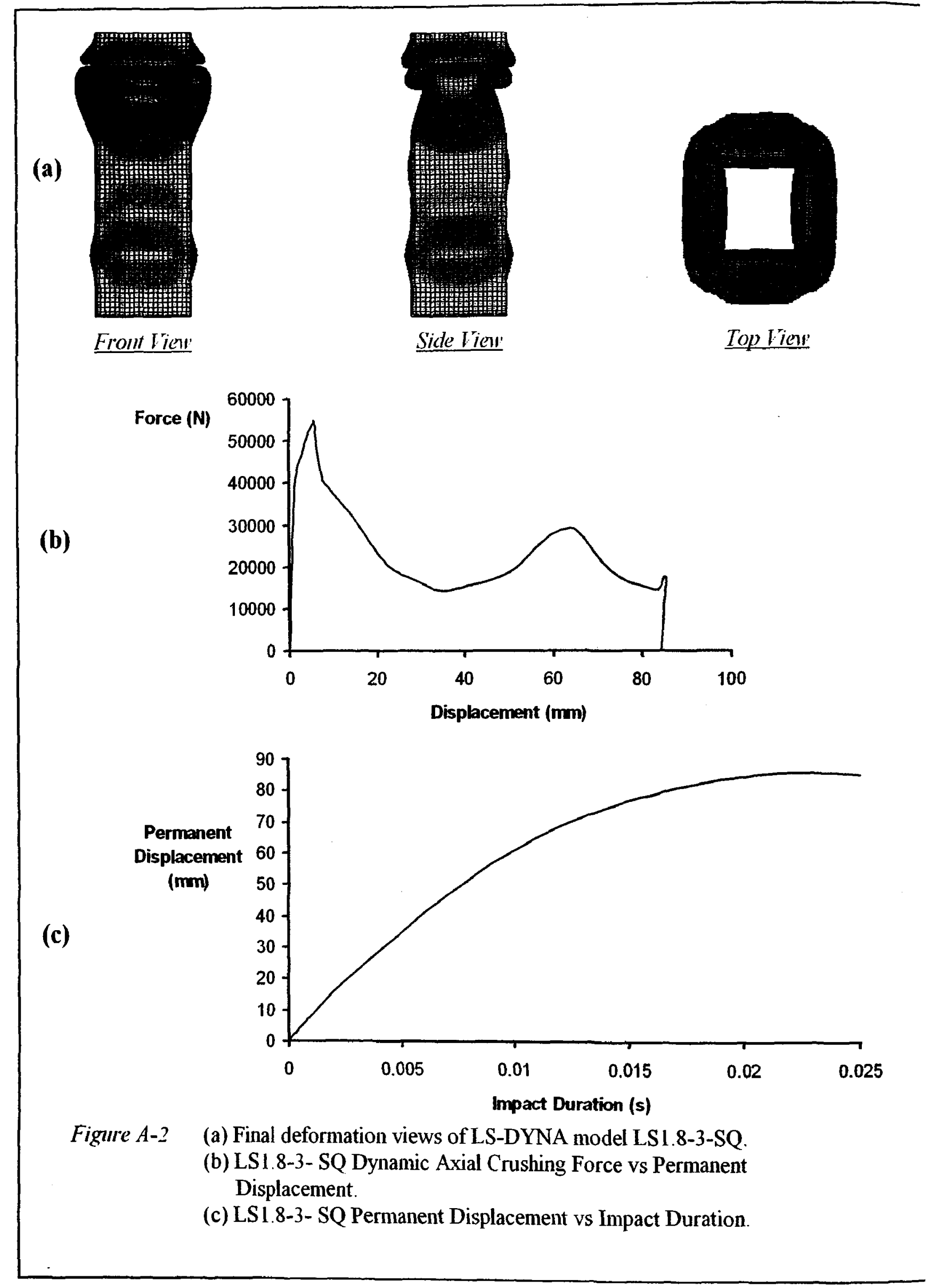


a)

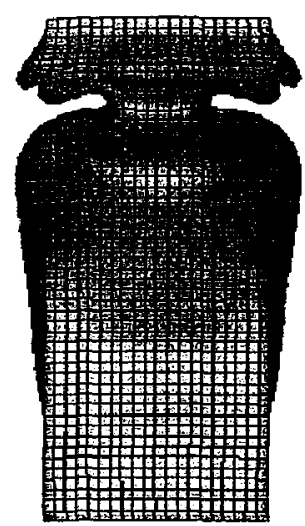

Front T'ielv'

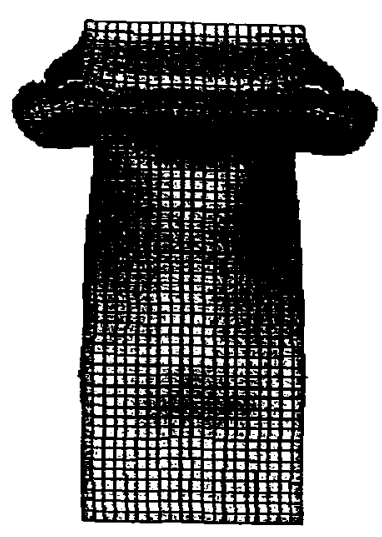

Side Iriew:

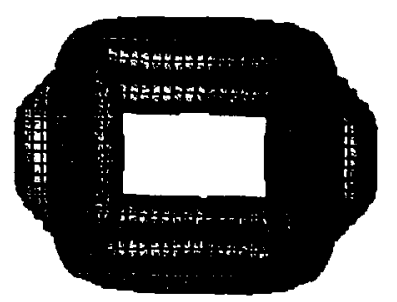

Top liew:

(b)

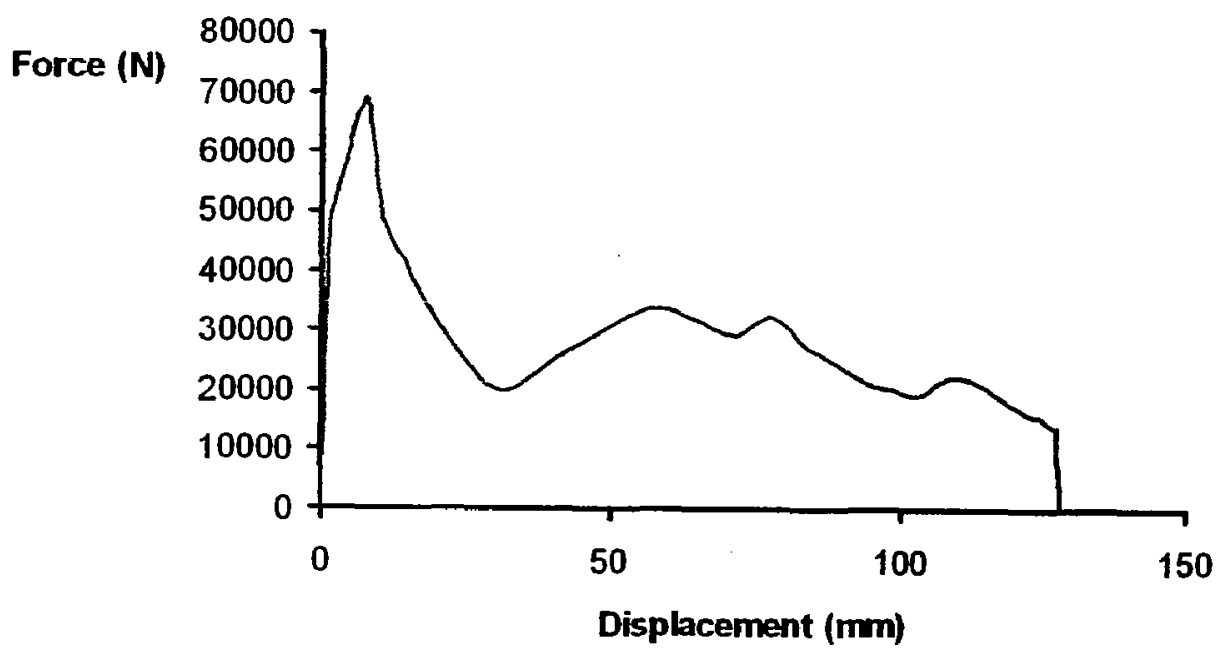

(c)

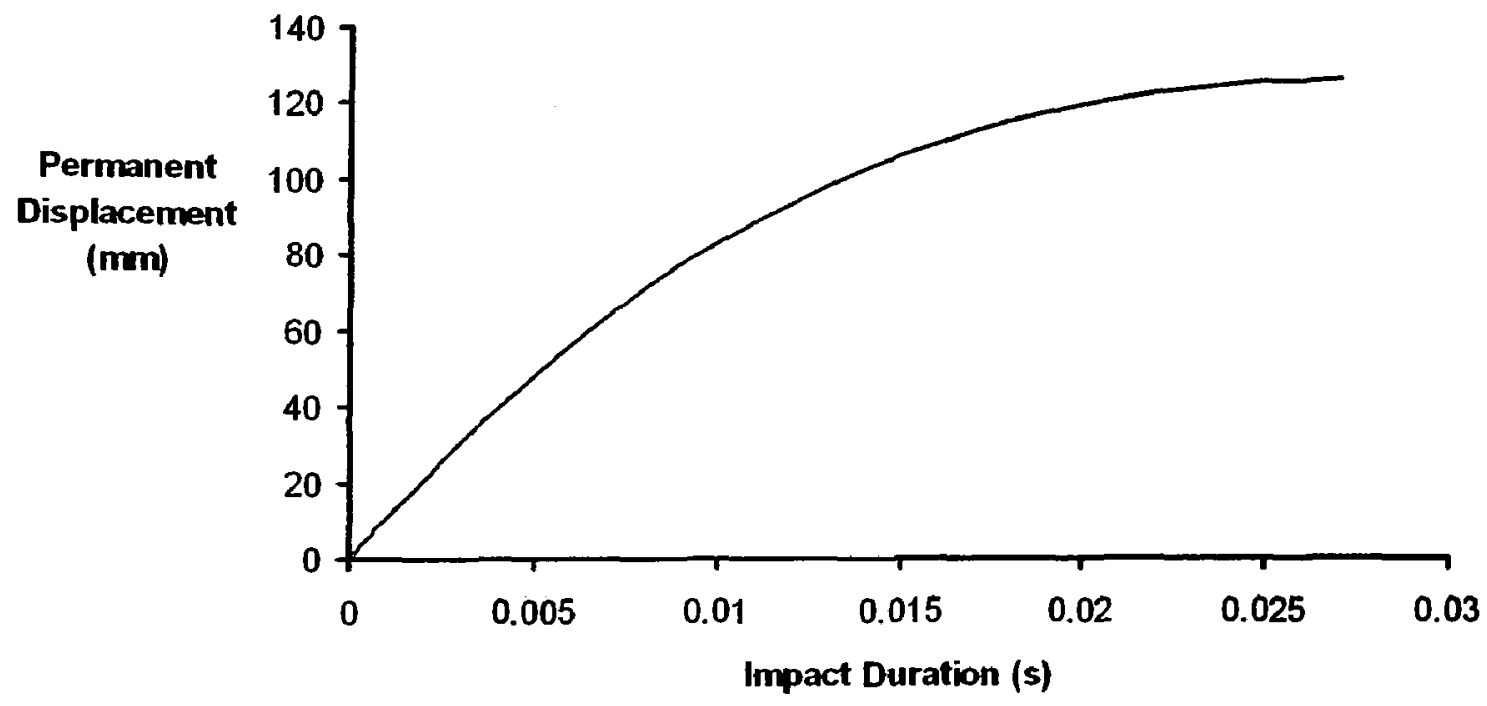

re A-3 (a) Final deformation views of LS-DYNA model LS2.0-4-SQ

(b) LS2.0-4-SQ Dynamic Axial Crushing Force vs Permanent Displacement.

(c) LS2.0-4-SQ Permanent Displacement vs Impact Duration. 
(a)

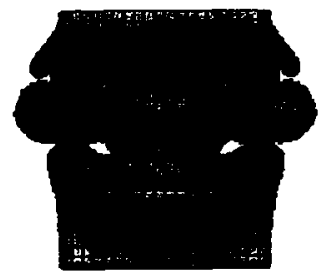

Front View

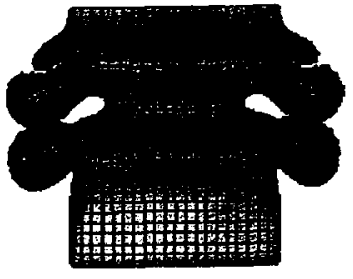

Side Vien:

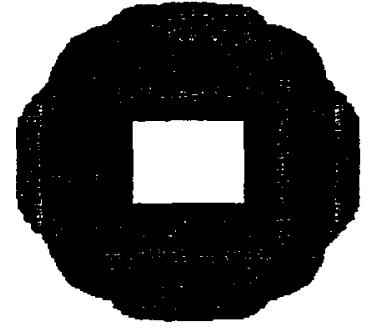

Top View

(b)

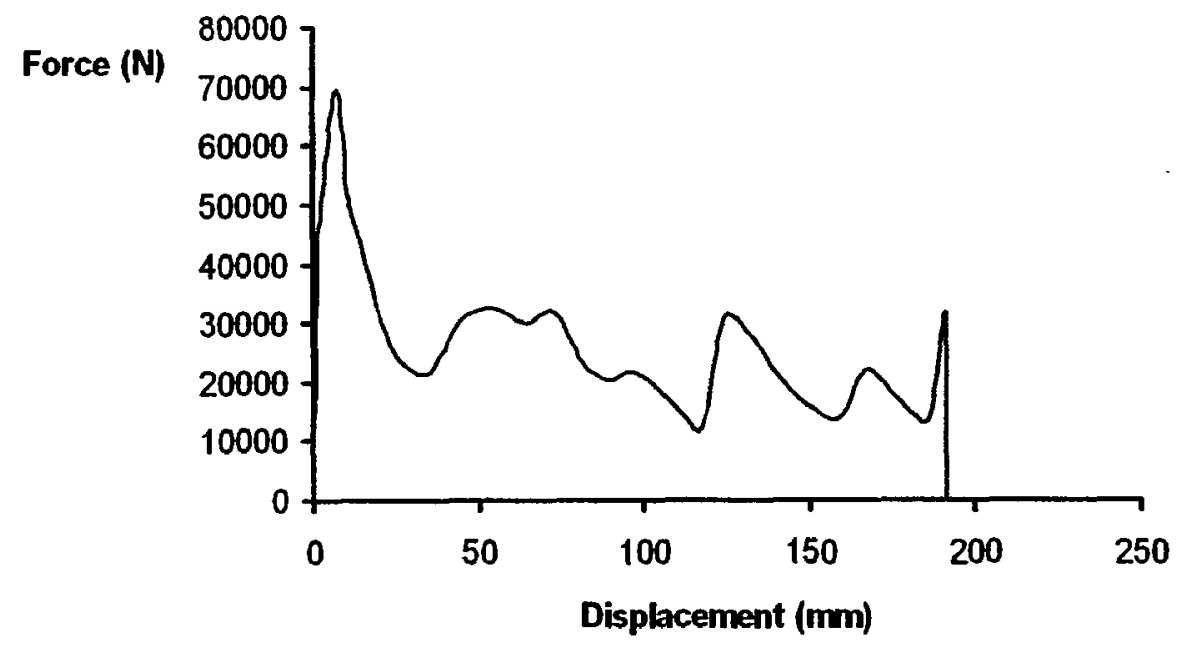

(c)

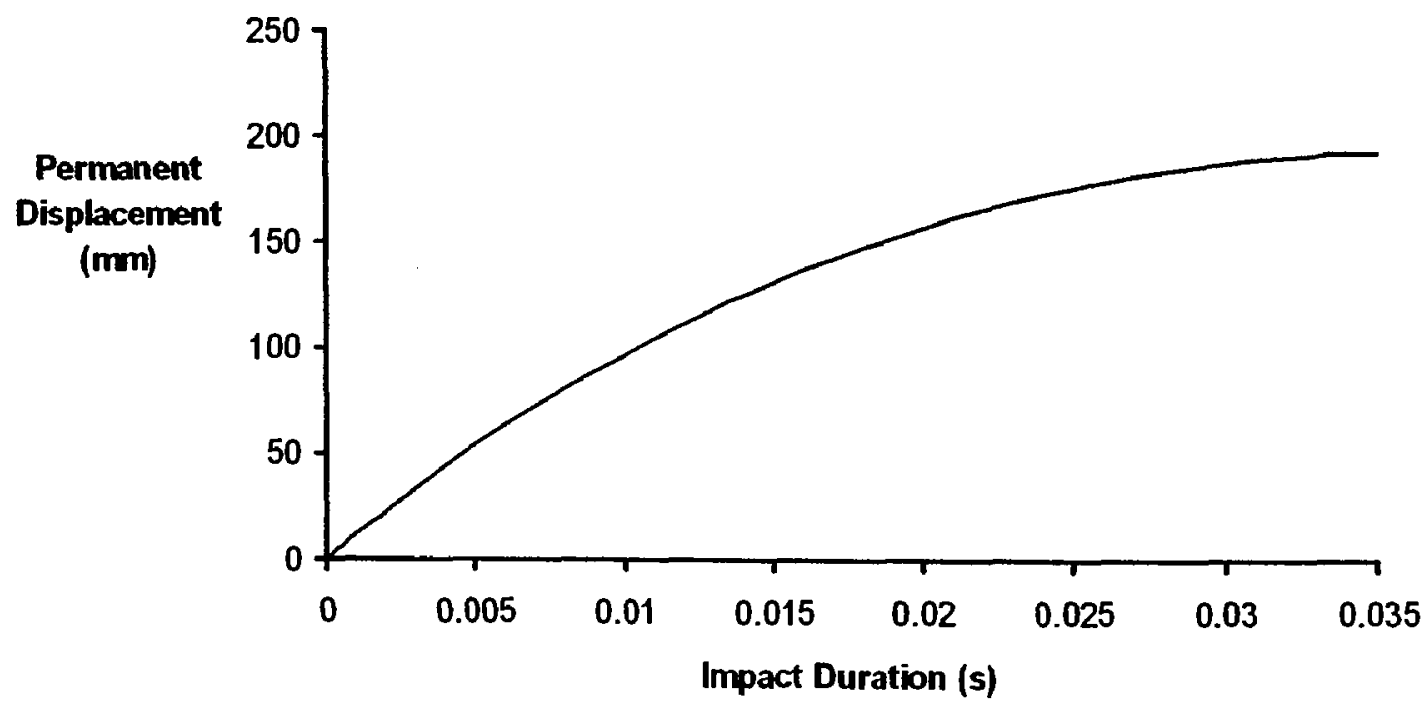

Figure $A-t \quad$ (a) Final deformation views of LS-DYNA model LS2.0-5-SQ.

(b) LS2.0-5-SQ Dynamic Axial Crushing Force vs Permanent Displacement.

(c) LS2.0-5- SQ Permanent Displacement vs Impact Duration. 
1)
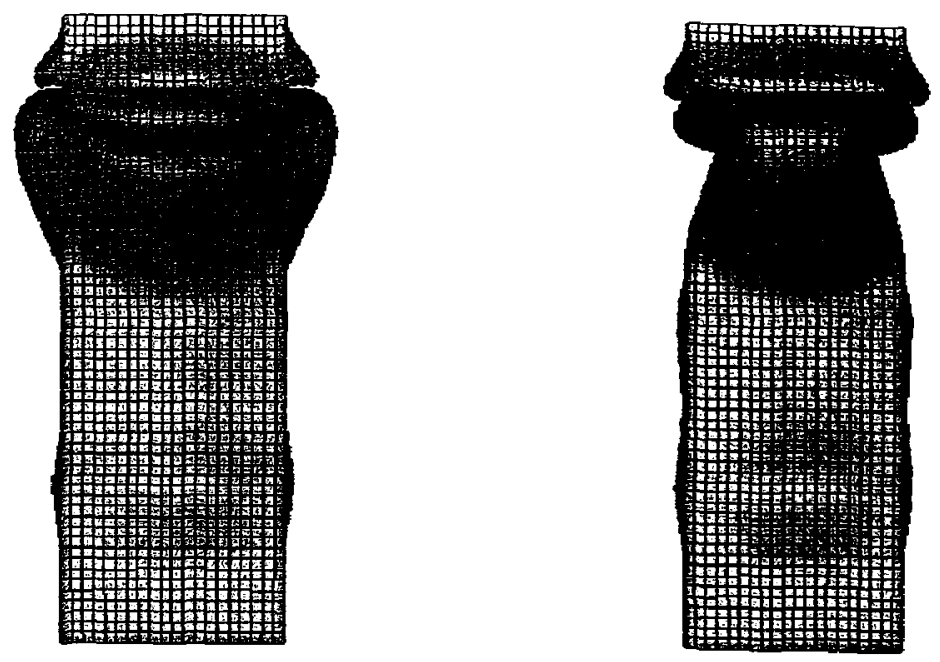

Front Vien:

Side Vien:

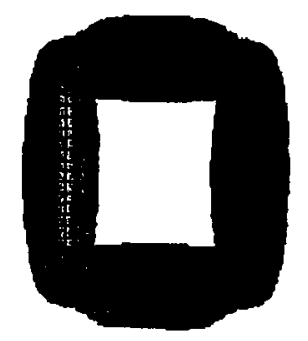

Top Vien:

b)
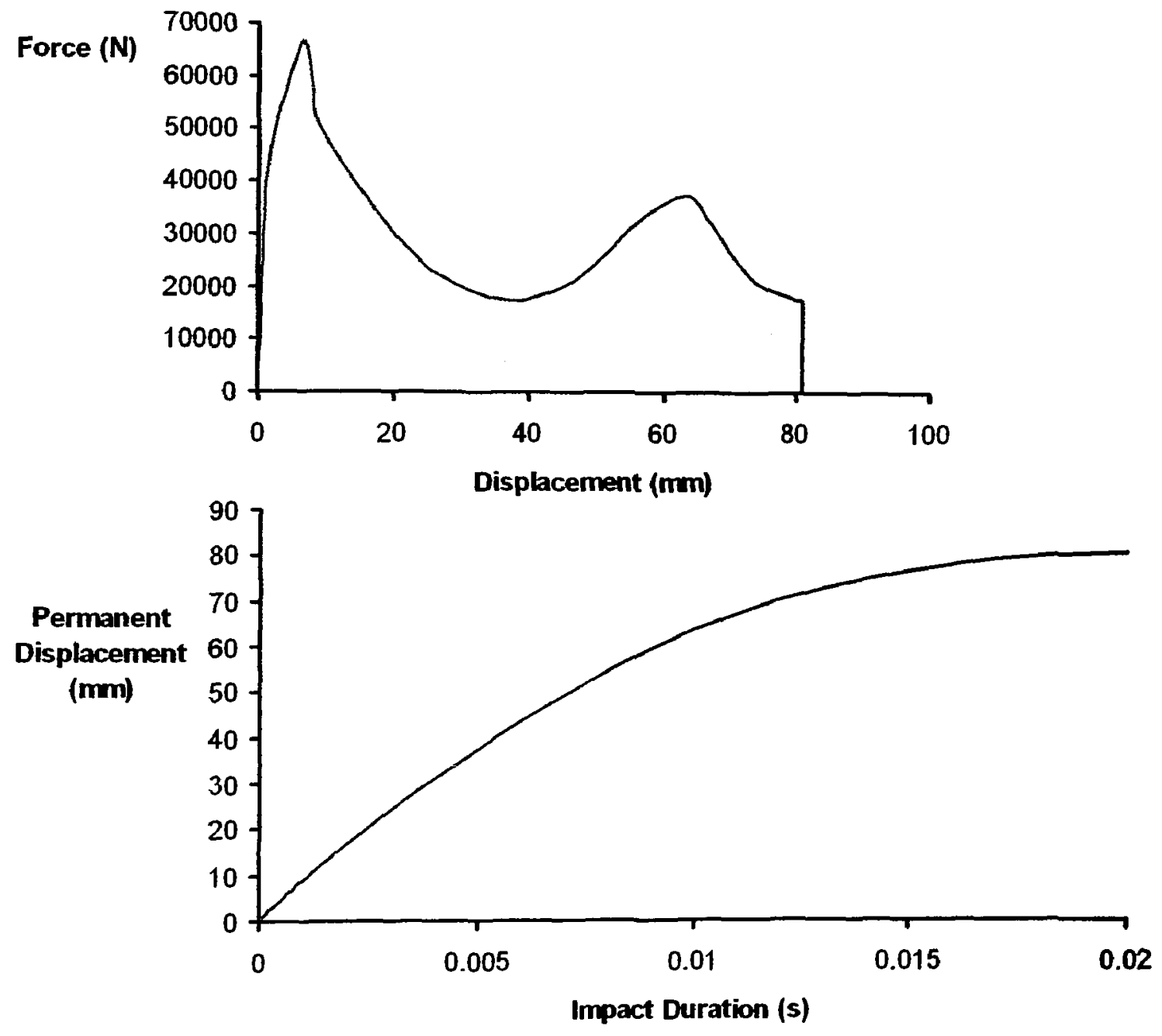

"e $A-5$ (a) Final deformation views of LS-DYNA model LS2.0-6-SQ.

(b) LS2.0-6- SQ Dynamic Axial Crushing Force vs Permanent Displacement.

(c) LS2.0-6- SQ Permanent Displacement vs Impact Duration. 


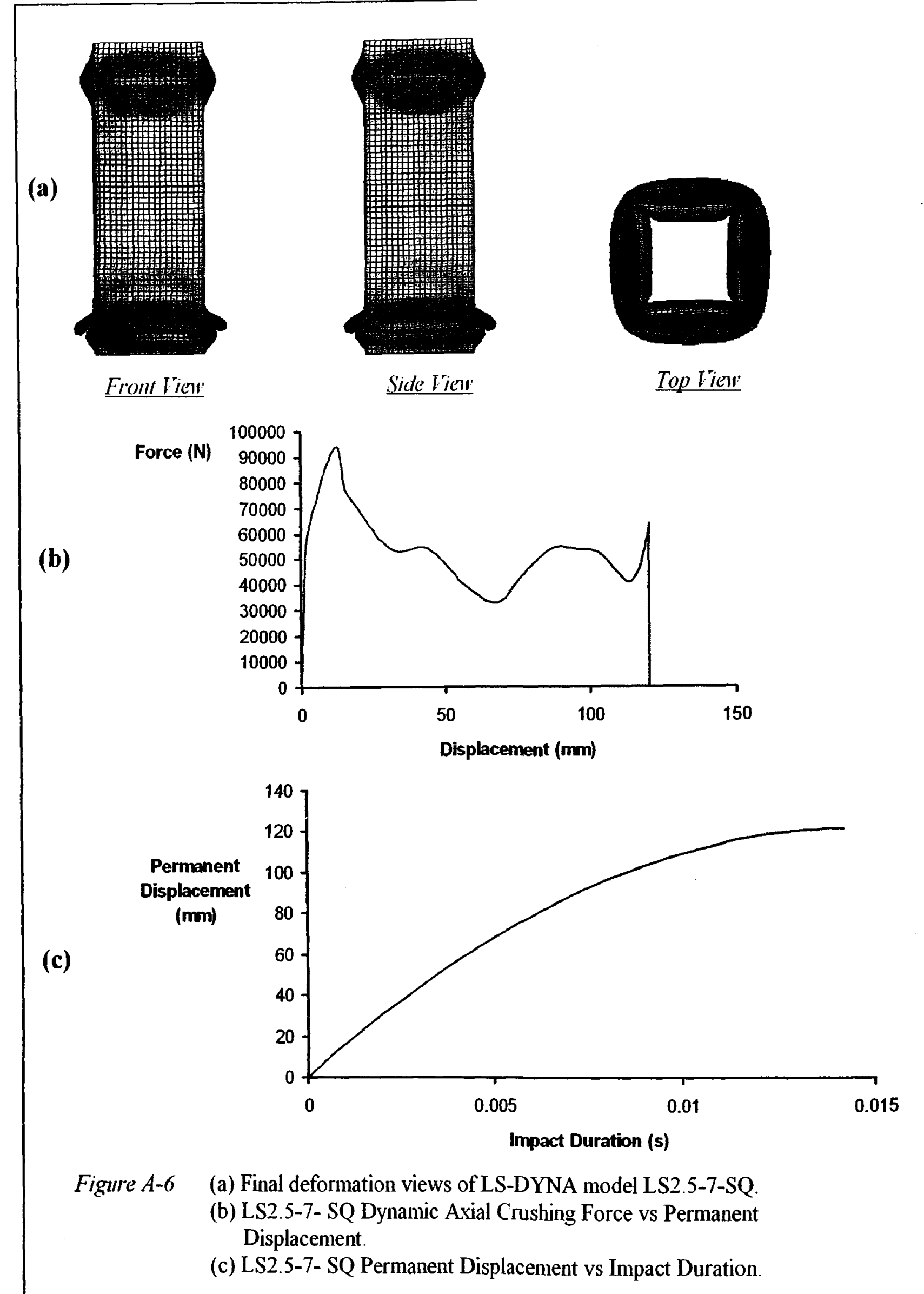


1)
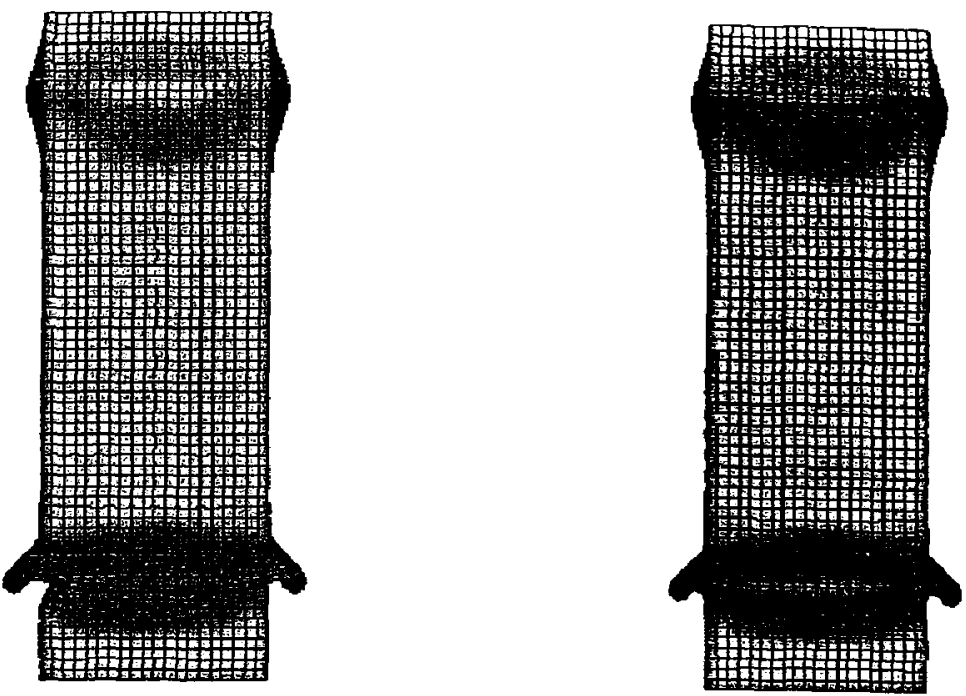

Front Vien:

(b) Force (N)

Side Vien:

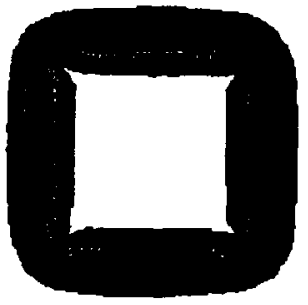

Top Vich:

(c)
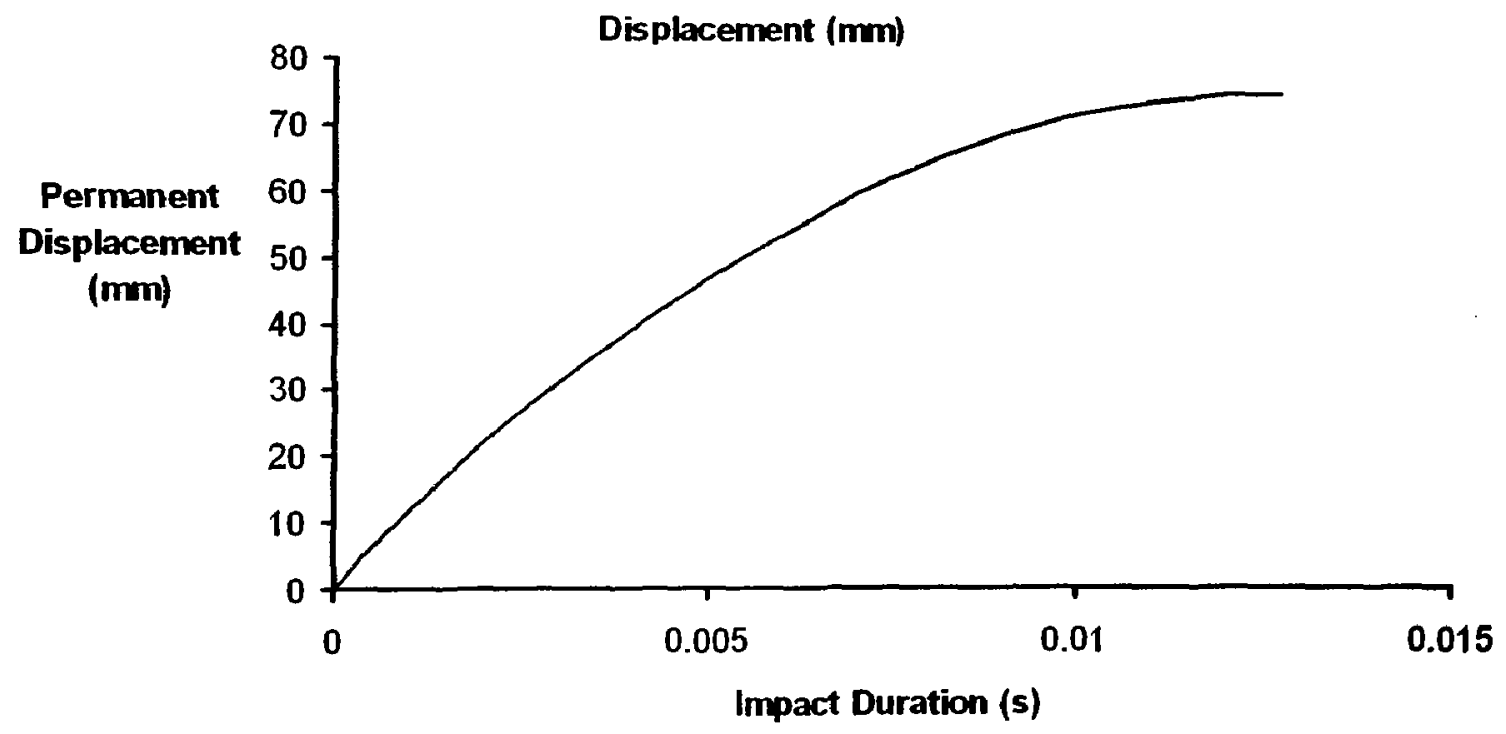

re $A-7$ (a) Final deformation views of LS-DYNA model LS2.5-8-SQ.

(b) LS2.5-8- SQ Dynamic Axial Crushing Force vs Permanent Displacement.

(c) LS2.5-8- SQ Permanent Displacement vs Impact Duration. 
(a)

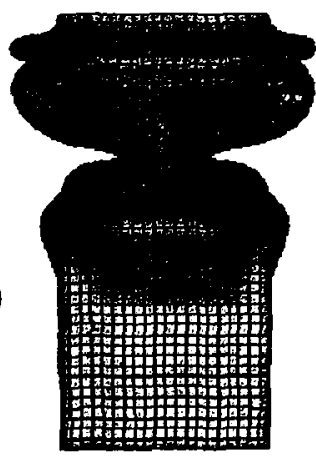

Front lient

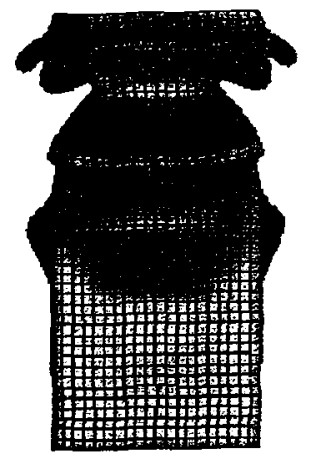

Side Vient:

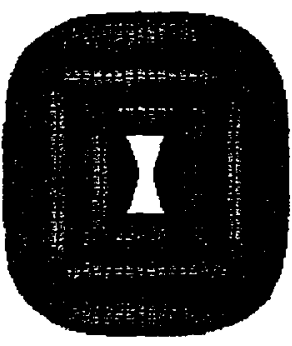

Top Vien

(b)

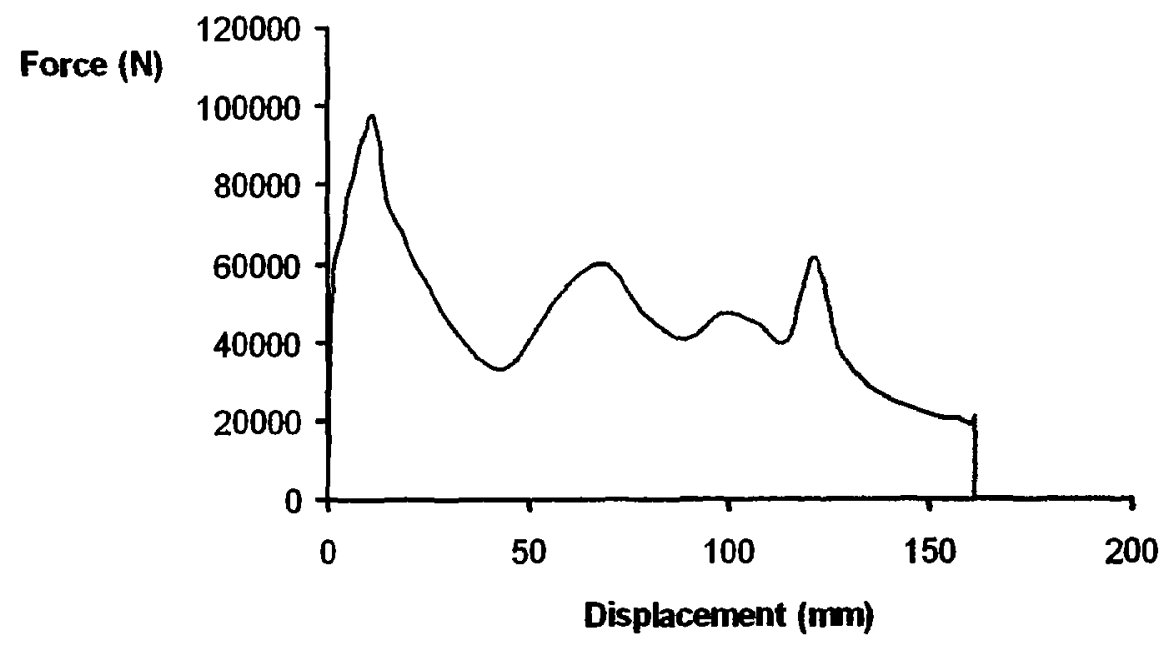

(c)

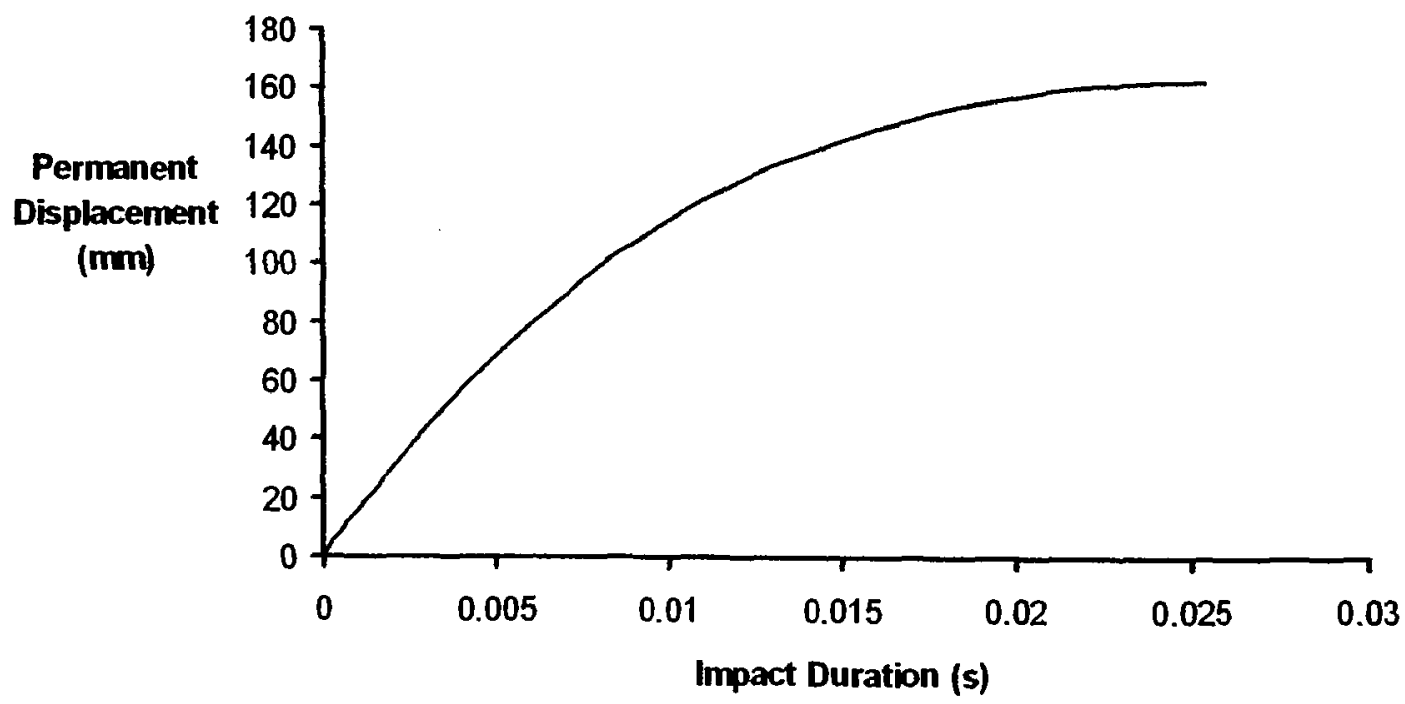

Figire A-8 (a) Final deformation views of LS-DYNA model LS2.5-9-SQ.

(b) LS2.5-9- SQ Dynamic Axial Crushing Force vs Permanent Displacement.

(c) LS2.5-9-SQ Permanent Displacement vs Impact Duration. 
1)

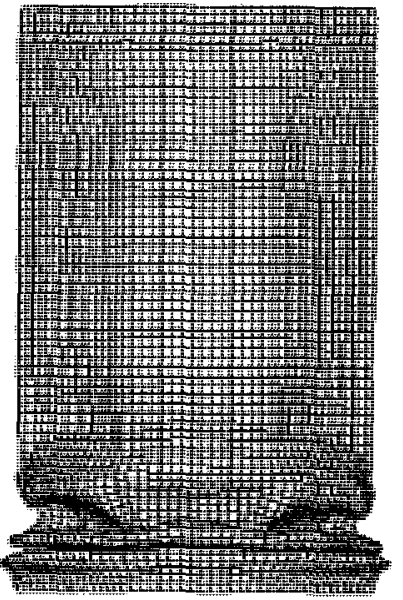

Front lien

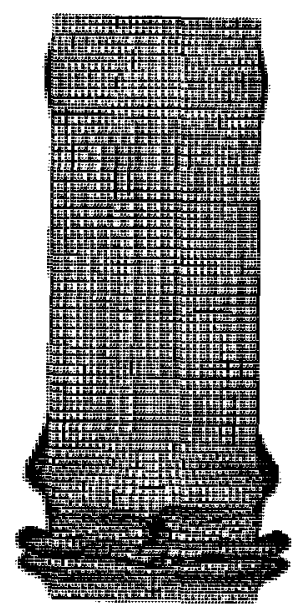

Side liew:

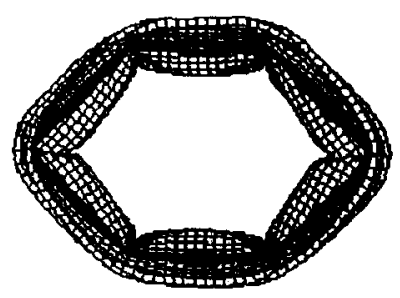

Top Iien:

b)
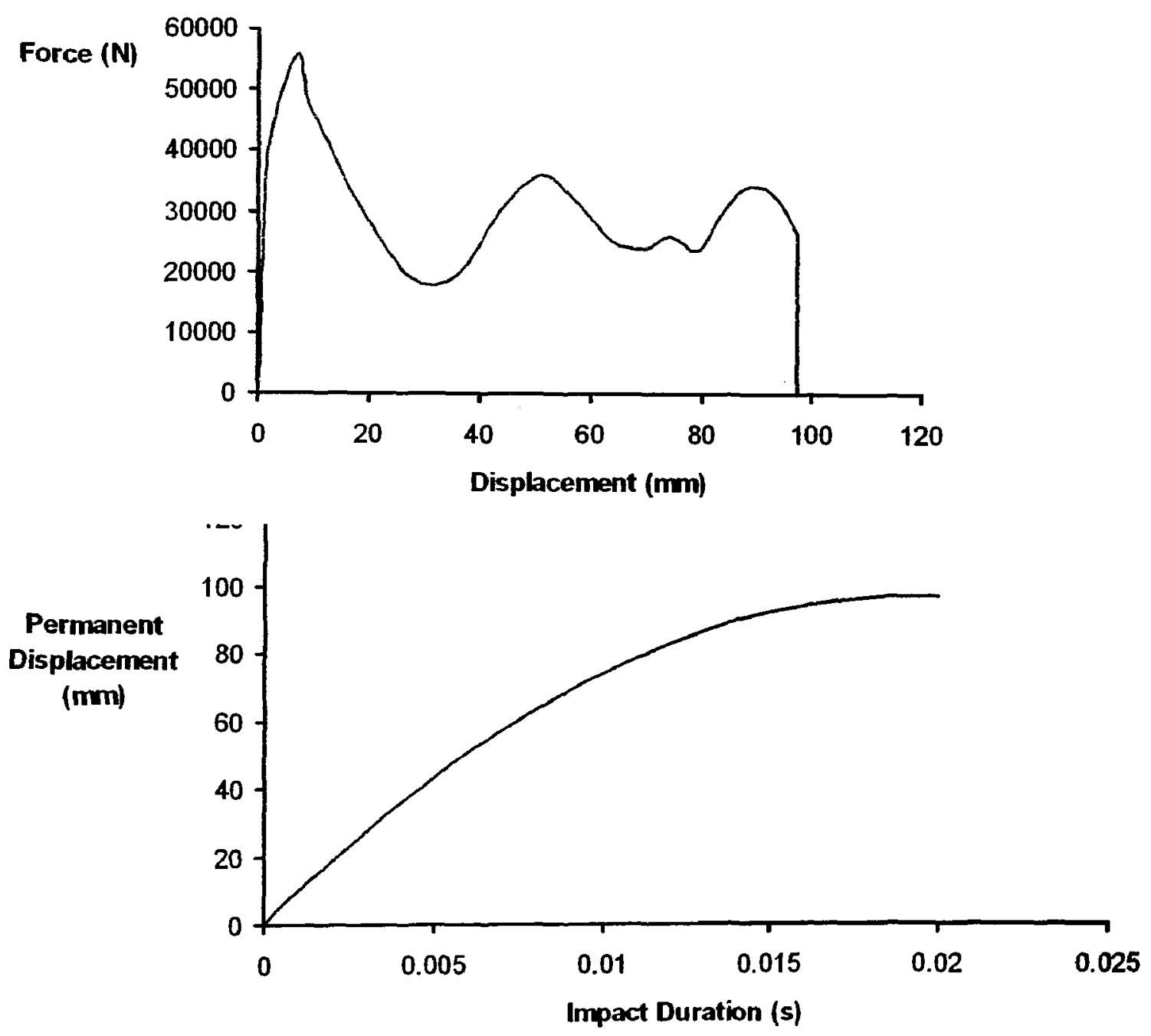

re A-9 (a) Final deformation views of LS-DYNA model LS1 8-2-HEX.

(b) LS 1.8-2-HEX Dynamic Axial Crushing Force vs Permanent Displacement.

(c) LS1.8-2-HEX Permanent Displacement vs Impact Duration. 
(a)

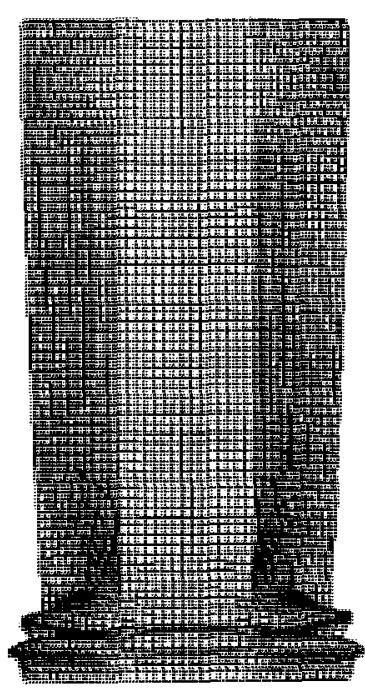

Front Iriew:

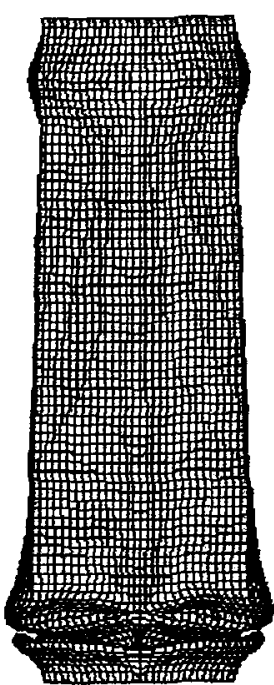

Side Iien:

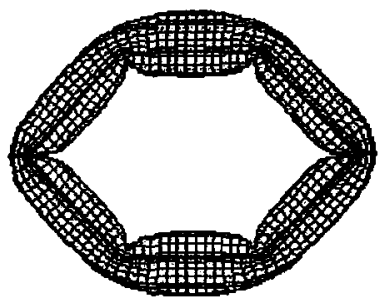

Top Vien:

(b)

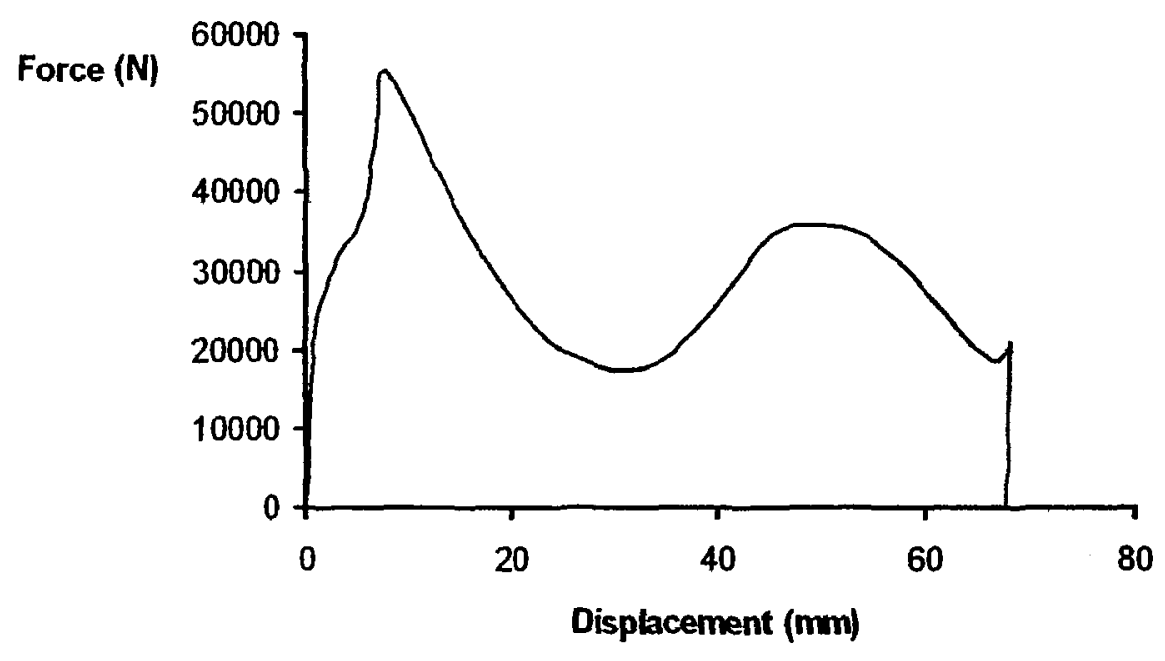

(c)

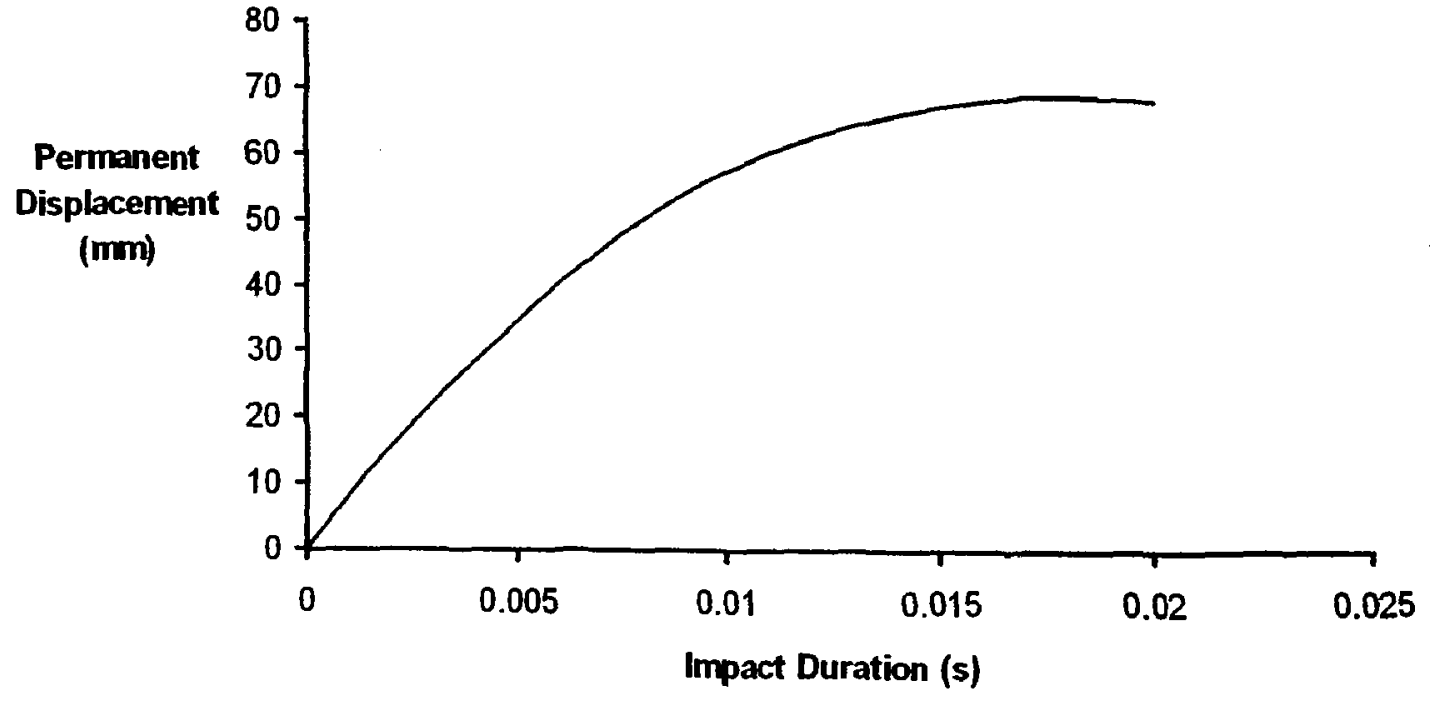

Figure A-10 (a) Final deformation views of LS-DYNA model LS1 8-3-HEX.

(b) LS1.8-3-HEX Dynamic Axial Crushing Force vs Permanent Displacement.

(c) LSI.8-3-HEX Permanent Displacement vs Impact Duration. 
1)

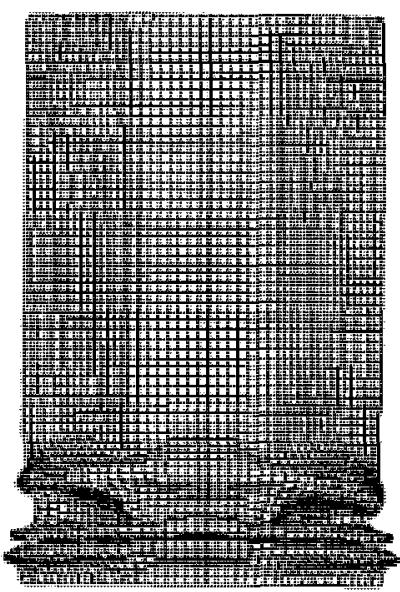

Front Vien:
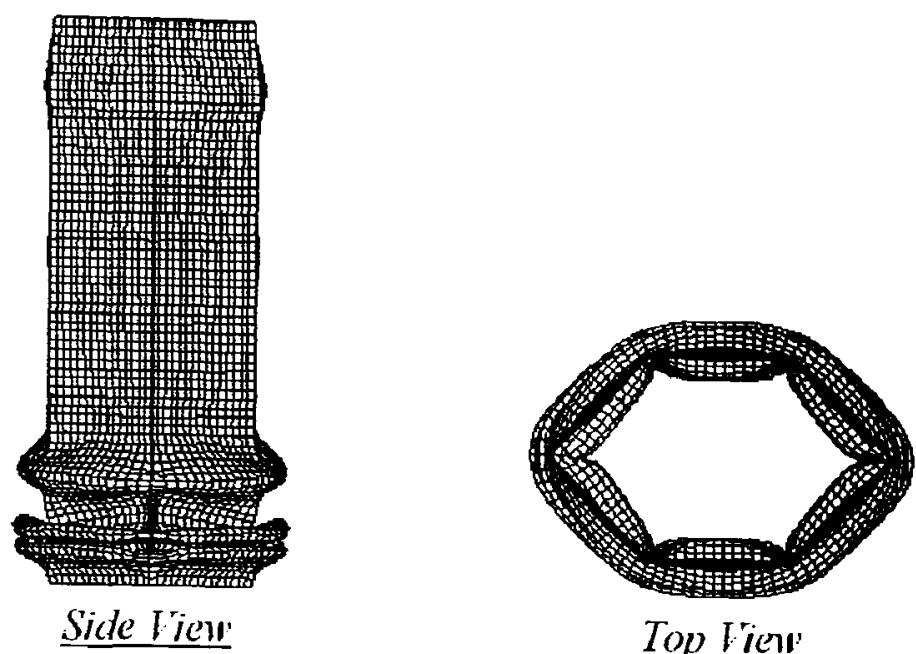

Top Vien:
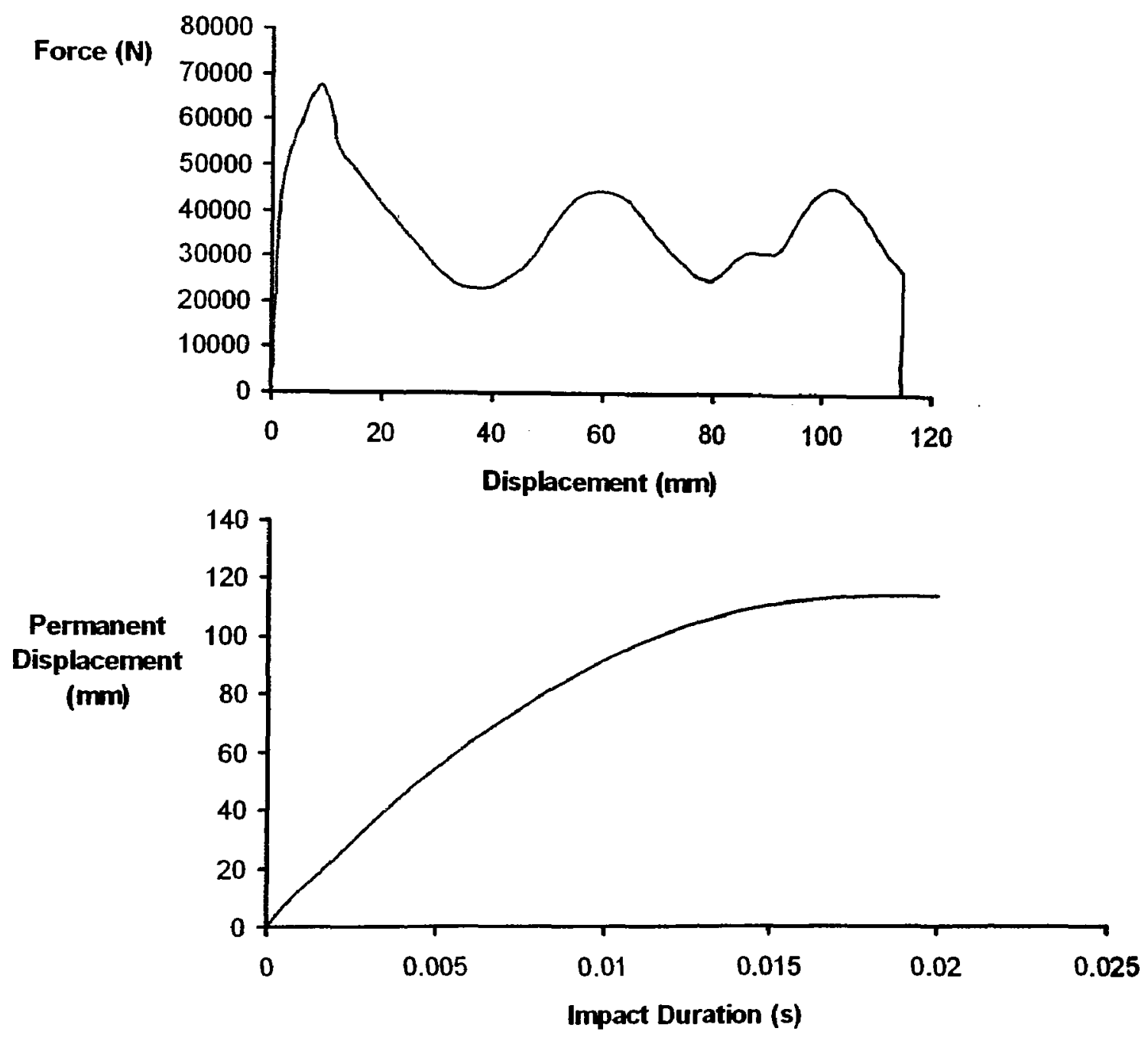

re A-II (a) Final deformation views of LS-DYNA model LS2.0-4-HEX.

(b) LS2.0-4-HEX Dynamic Axial Crushing Force vs Permanent Displacement.

(c) LS2.0-4-HEX Permanent Displacement vs Impact Duration. 
(a)

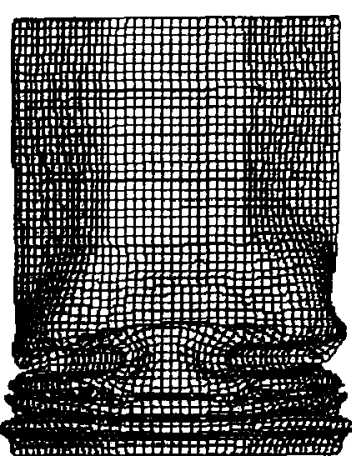

Front Vien:

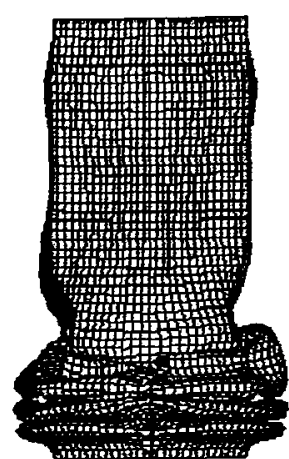

Side Vient

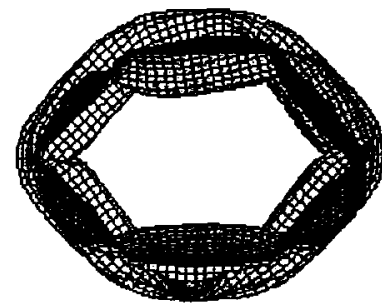

Top Vien'

(b)

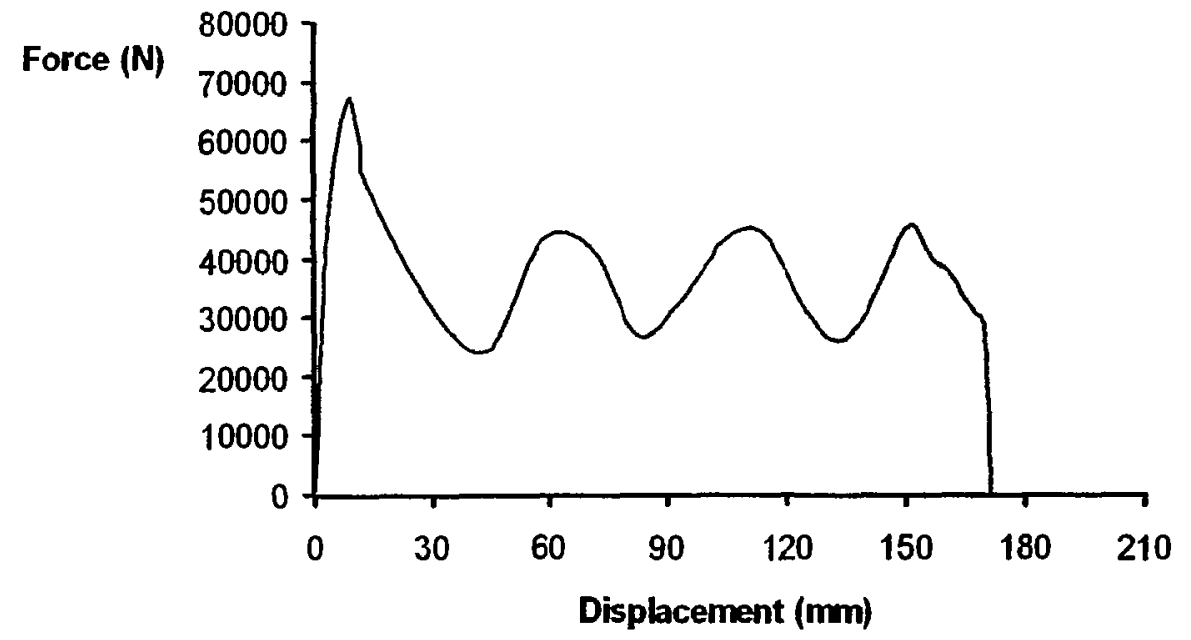

(c)

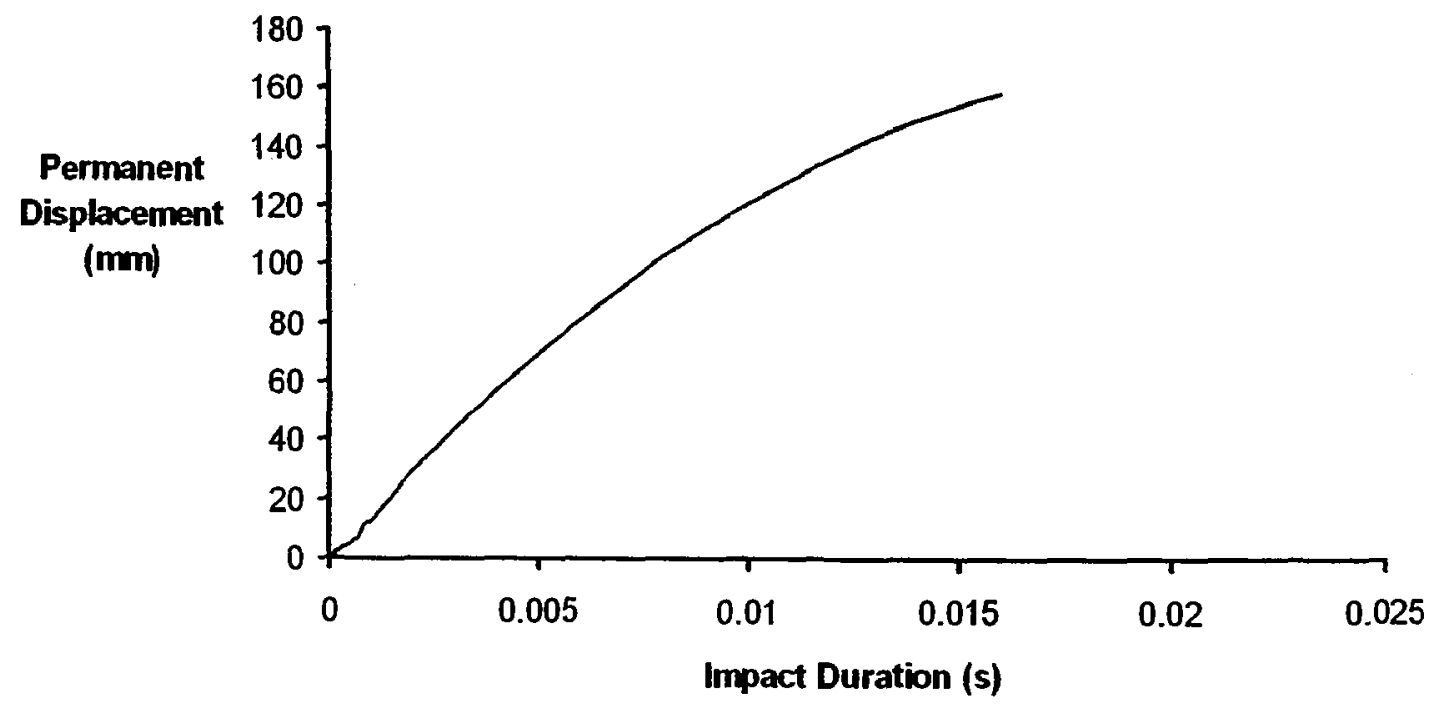

Figure A-12 (a) Final deformation views of LS-DYNA model LS2.0-5-HEX.

(b) LS2 0-6-HEX Dynamic Axial Crushing Force vs Permanent Displacement.

(c) LS2.0-6-HEX Permanent Displacement vs Impact Duration. 


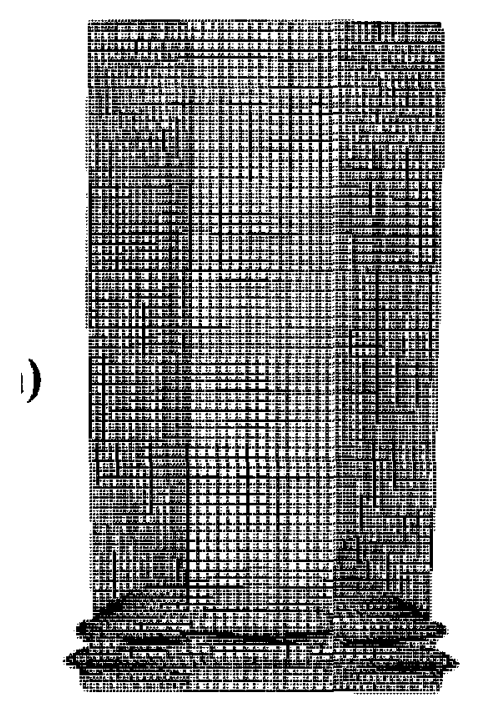

Front Vien:

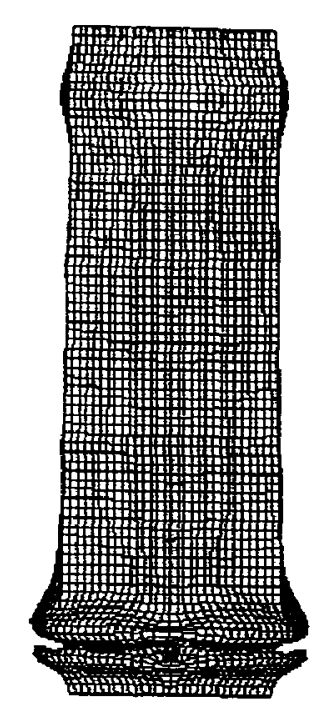

Side lien:

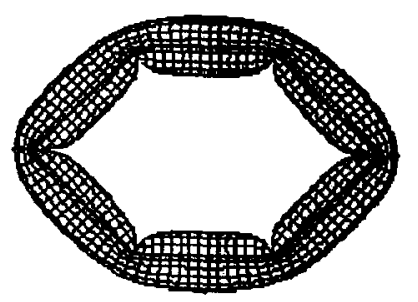

Top Vien

(b)
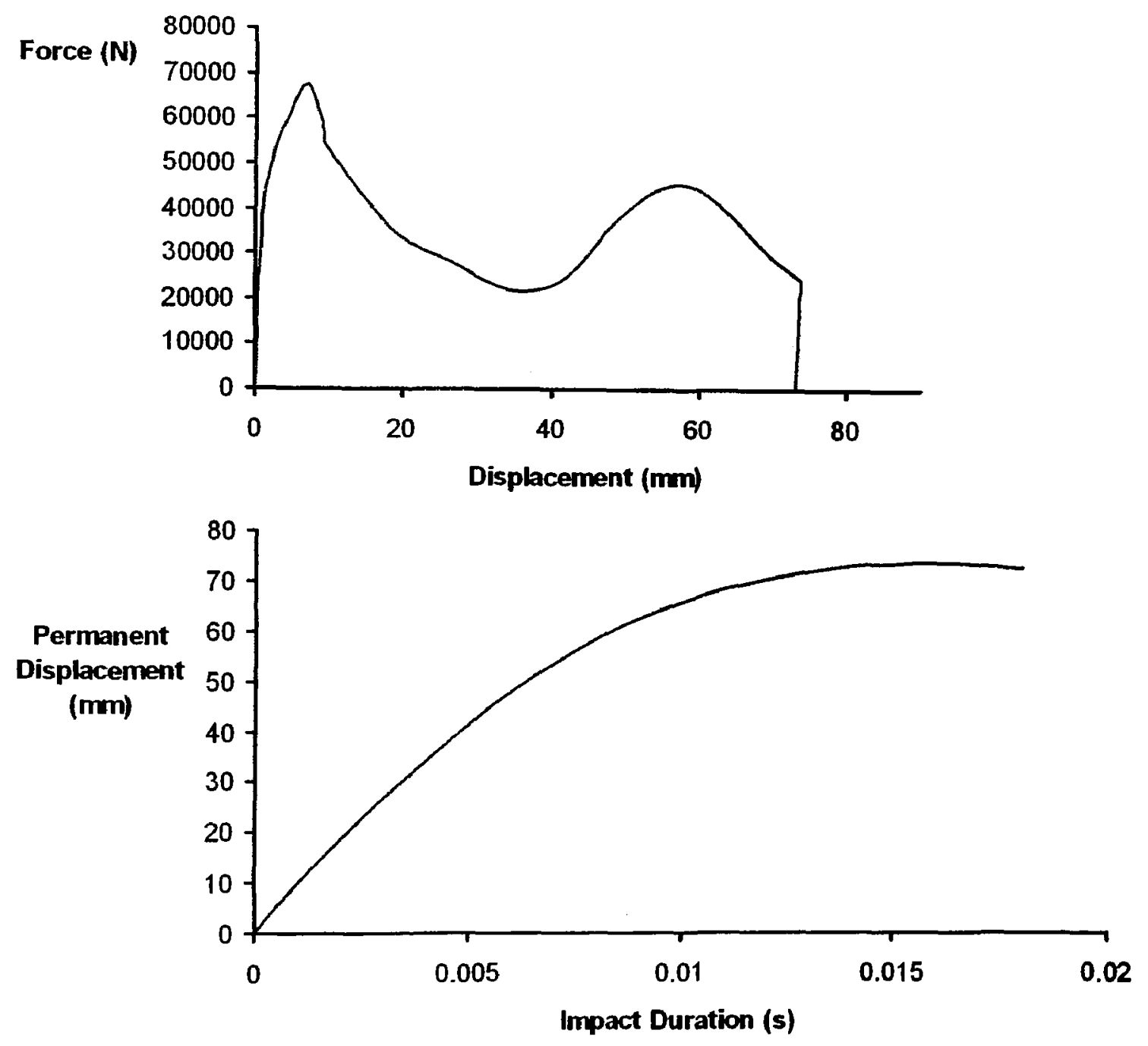

re $A-13$ (a) Final deformation views of LS-DYNA model LS2.0-6-HEX.

(b) LS2.0-6-HEX Dynamic Axial Crushing Force vs Permanent Displacement.

(c) LS2.0-6-HEX Permanent Displacement vs Impact Duration. 
(a)
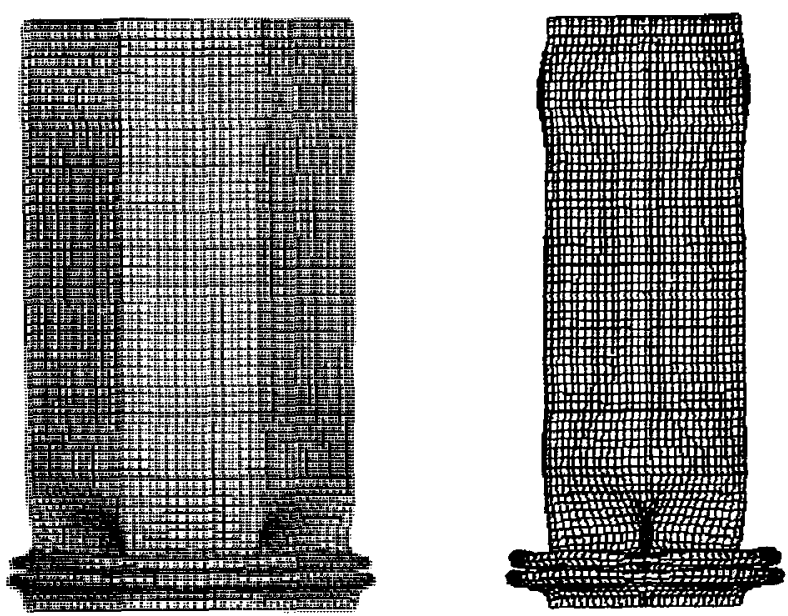

Frout liew

Side liew:

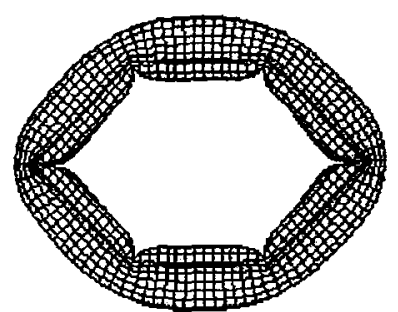

Top Vien:

(b)
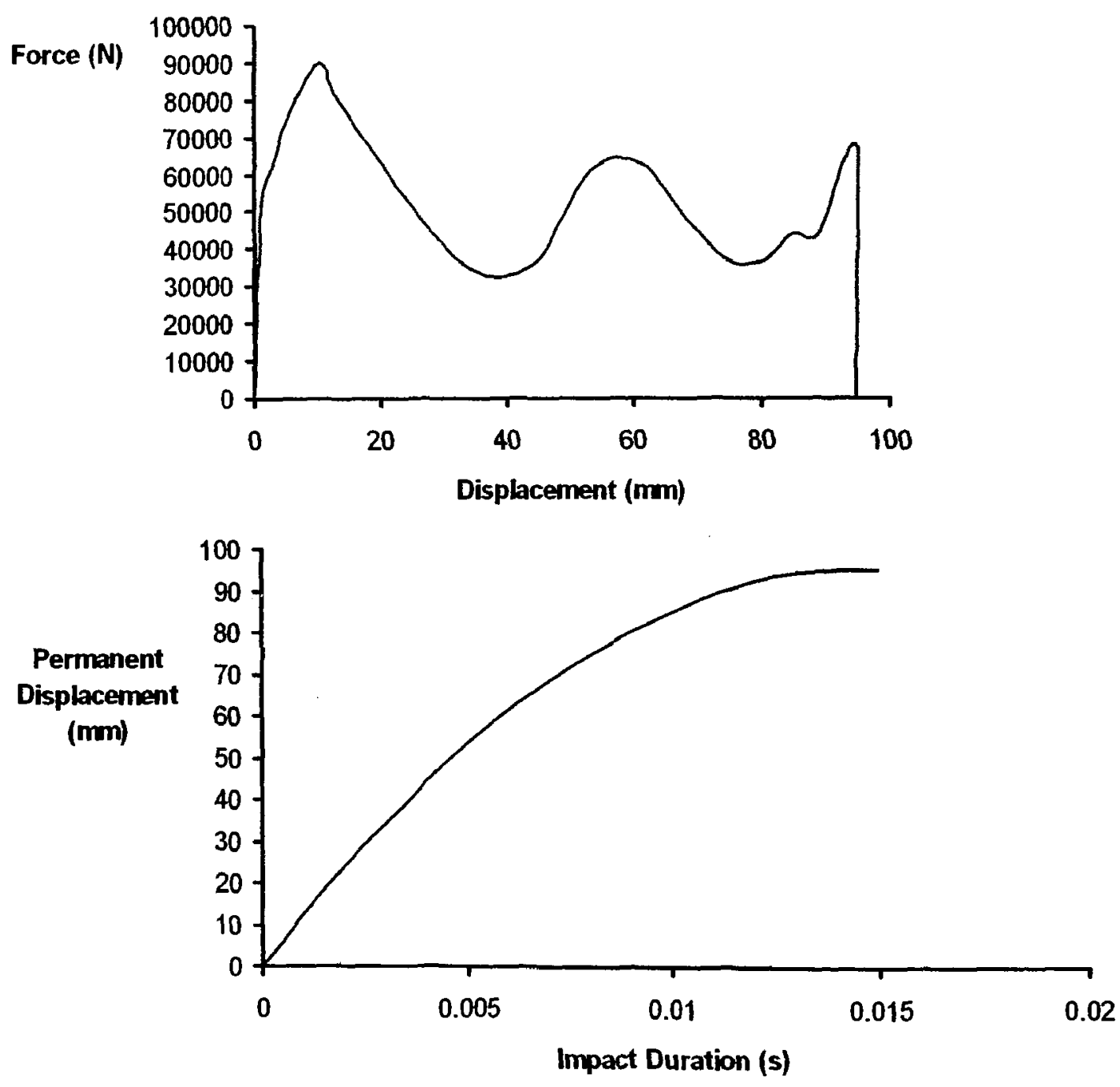

Figure A-1t (a) Final deformation views of LS-DYNA model LS2.5-7-HEX.

(b) LS2.5-7-HEX Dynamic Axial Crushing Force vs Permanent Displacement

(c) LS2.5-7-HEX Permanent Displacement vs Impact Duration. 
a)

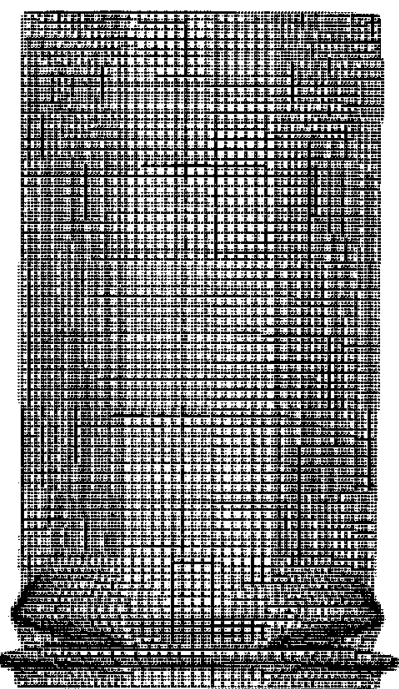

Front Vien:

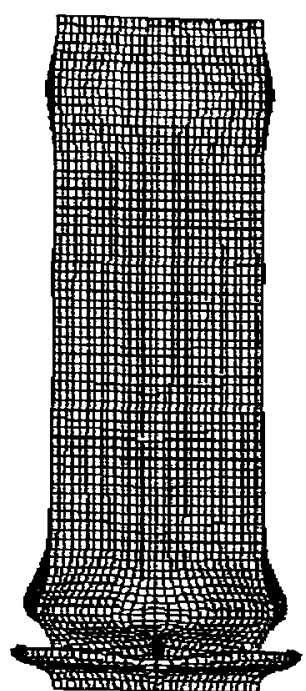

Sicle Iien'

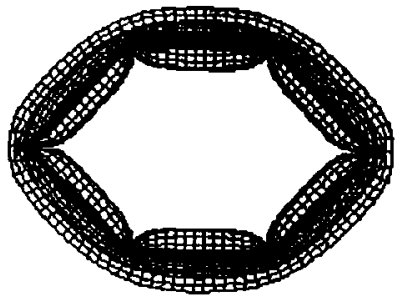

Top liew:

b)
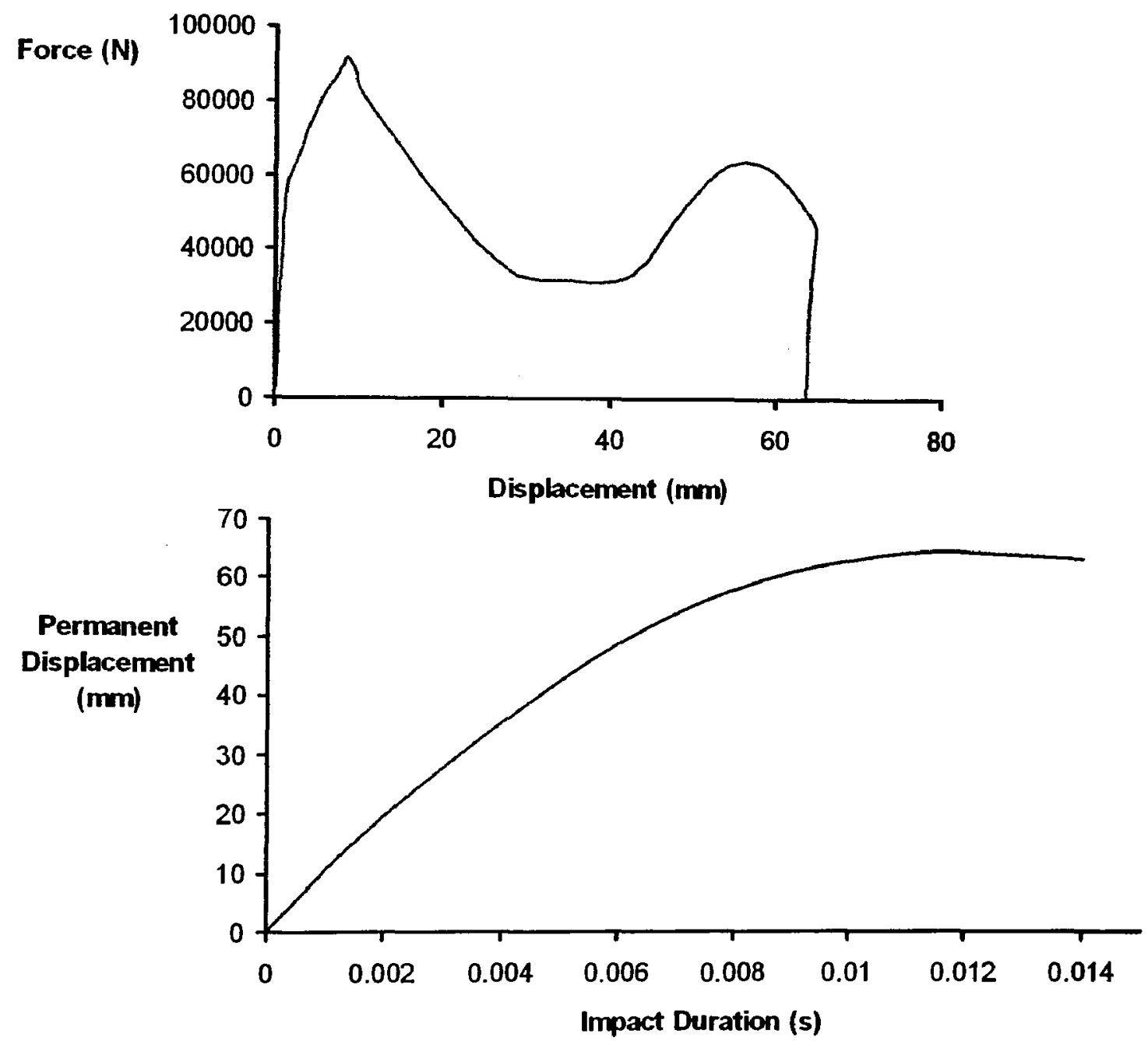

$e A-15$ (a) Final deformation views of LS-DYNA model LS2.5-8-HEX.

(b) LS2.5-8-HEX Dynamic Axial Crushing Force vs Permanent Displacement.

(c) LS2 5-8-HEX Permanent Displacement vs Impact Duration. 
(a)

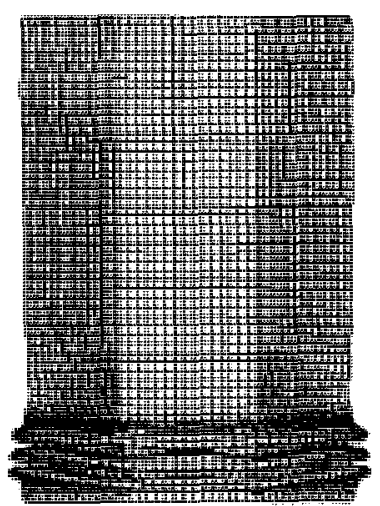

Front Iient

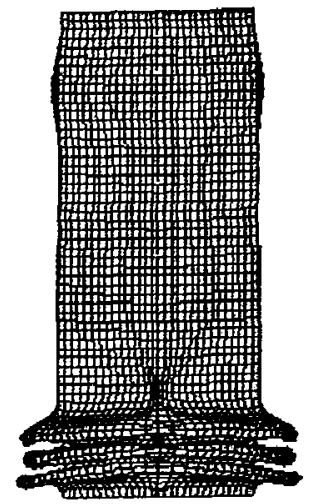

Sick Vien

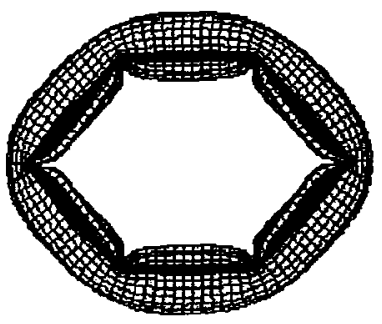

Top View:

(b)
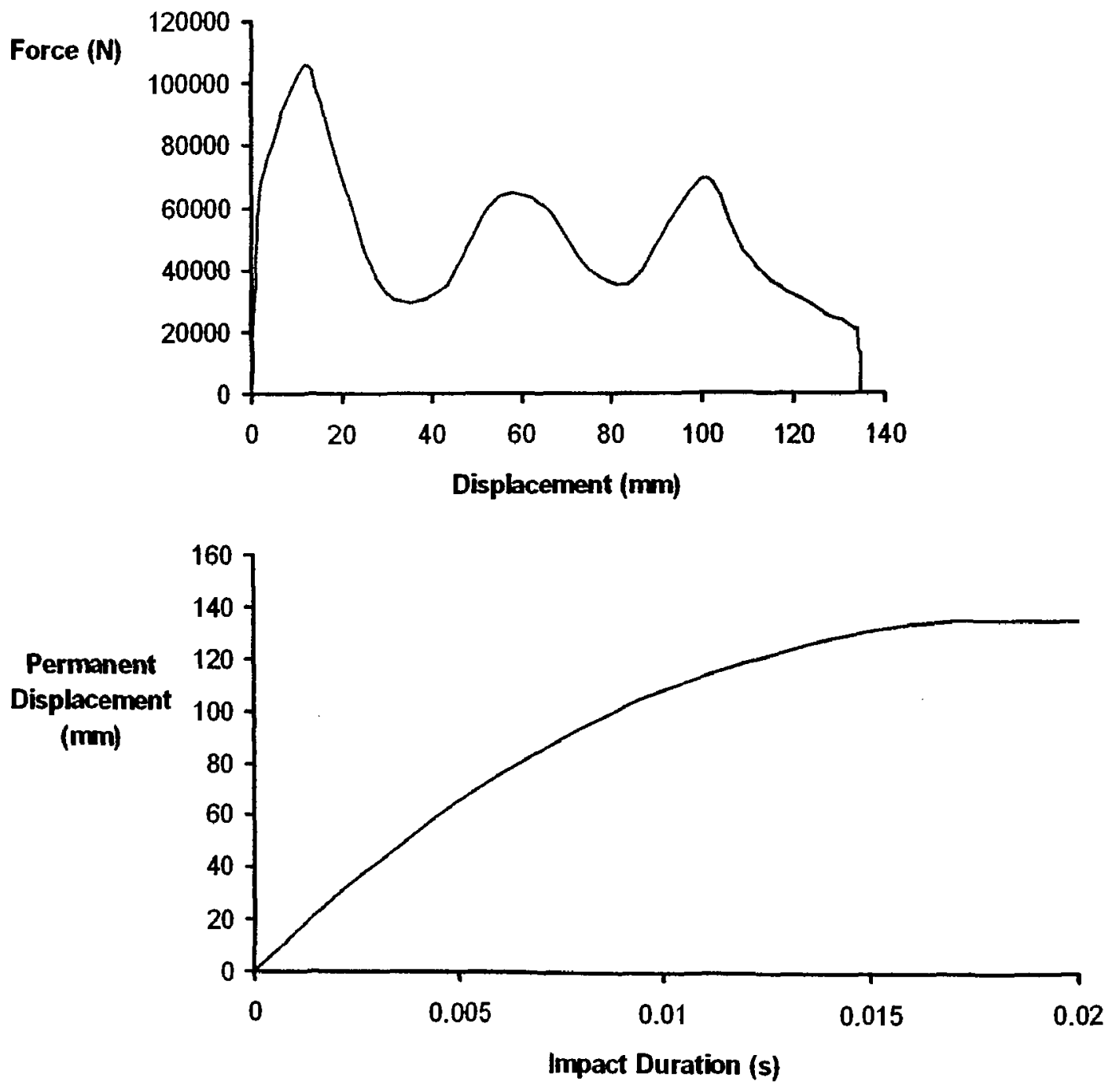

Figure A-16 (a) Final deformation views of LS-DYNA model LS2.5-9-HEX.

(b) LS2.5-9-HEX Dynamic Axial Crushing Force vs Permanent Displacement

(c) LS2.5-9-HEX Permanent Displacement vs Impact Duration. 
1)

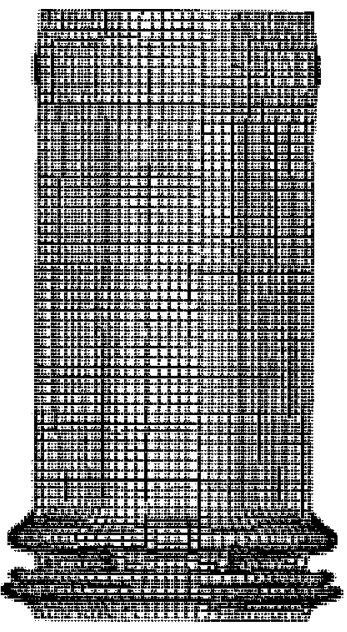

Front liew

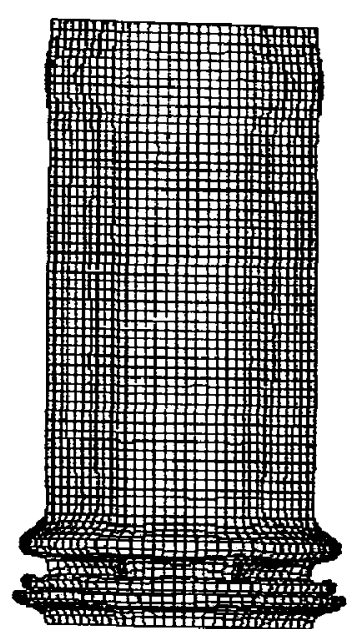

Side lien'

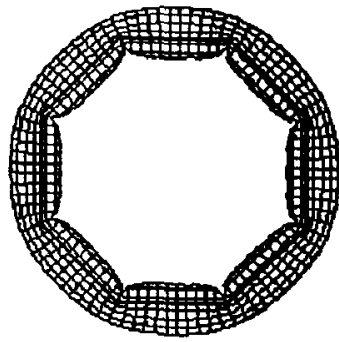

Top View'

b)

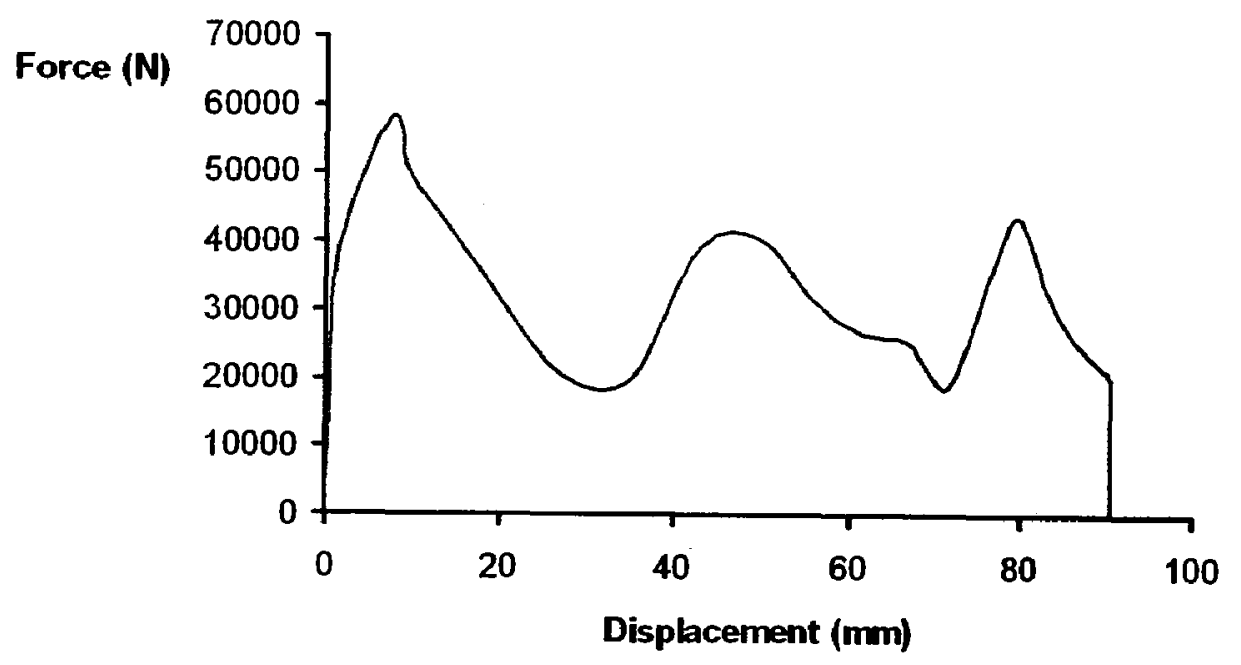

c)

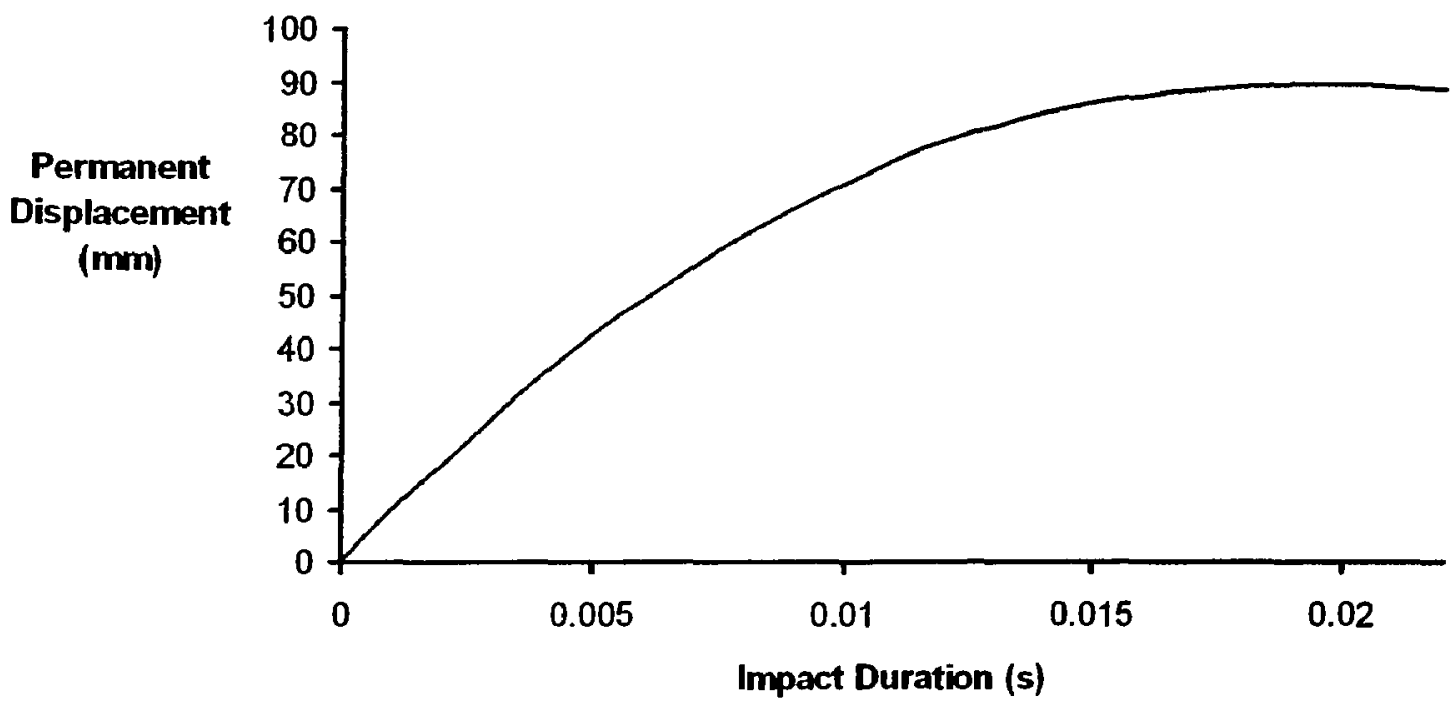

re A-17- (a) Final deformation views of LS-DYNA model LS1.8-2-OCT.

(b) LS1.8-2-OCT Dynamic Axial Crusling Force vs Permanent Displacement.

(c) LS 1.8-2-OCT Permanent Displacement vs Impact Duration. 
(a)

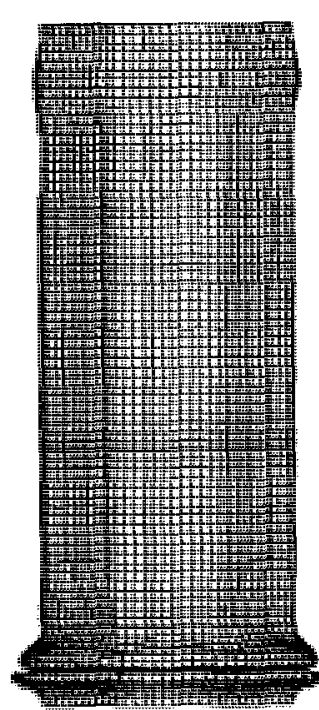

Front Vien:

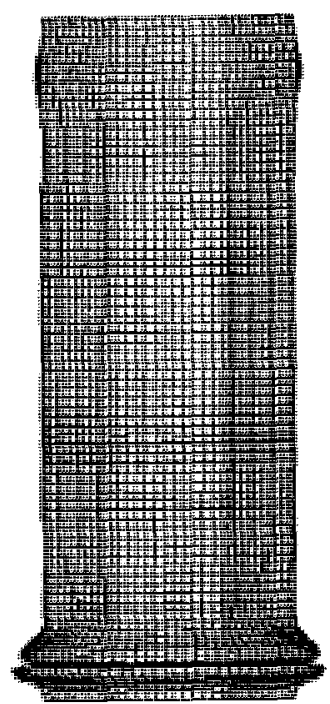

Side Vien:

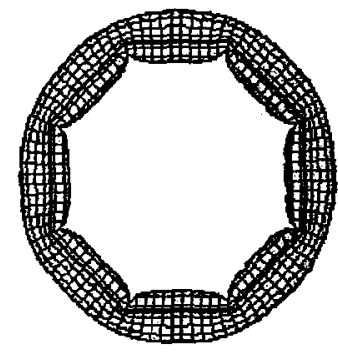

Top Vien:

(b)
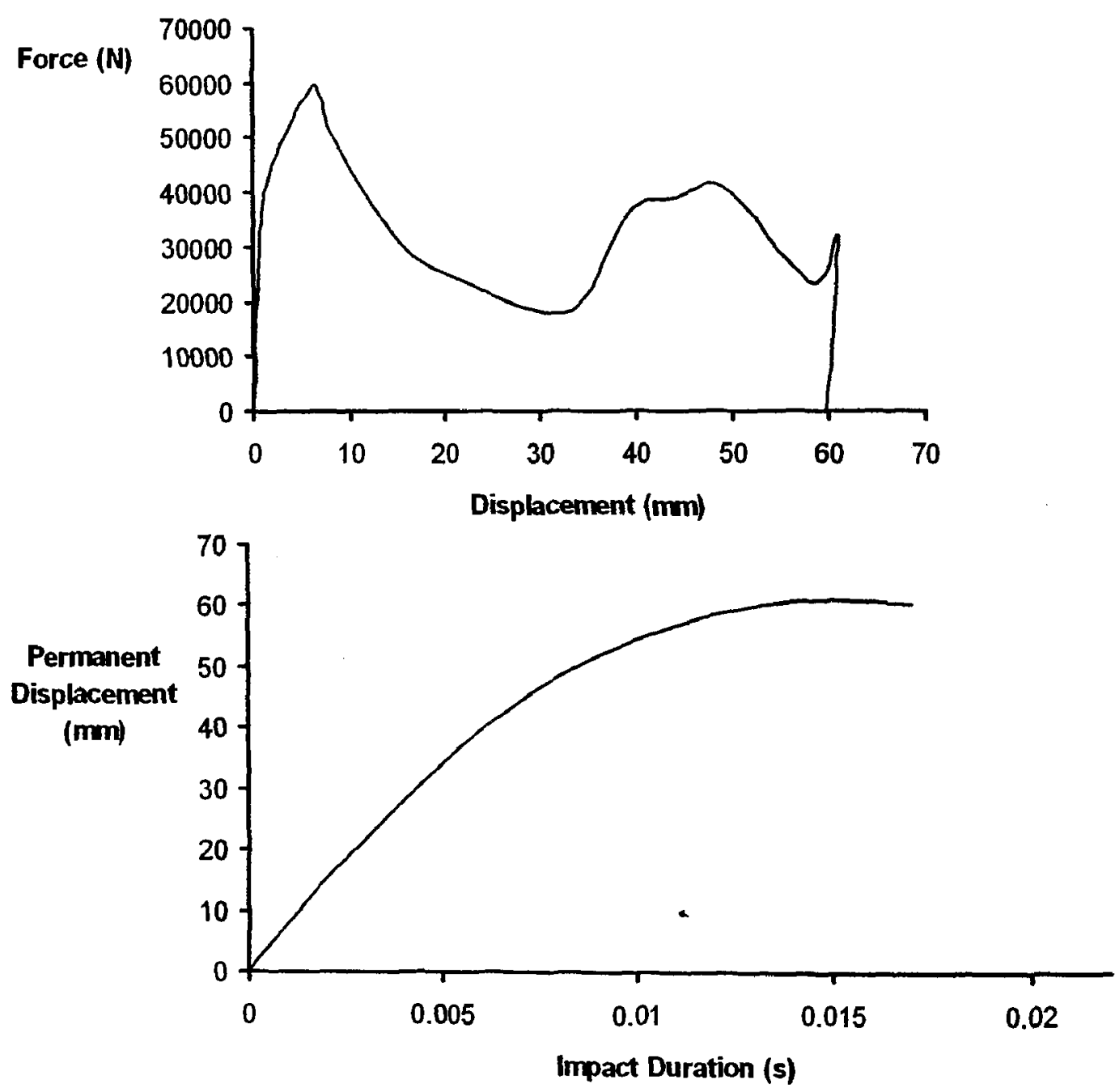

Figure A-18 (a) Final deformation views of LS-DYNA model LS1.8-3-OCT.

(b) LS1.8-3-OCT Dynamic Axial Crushing Force vs Permanent Displacement.

(c) LS1.8-3-OCT Permanent Displacement vs Impact Duration 
a)

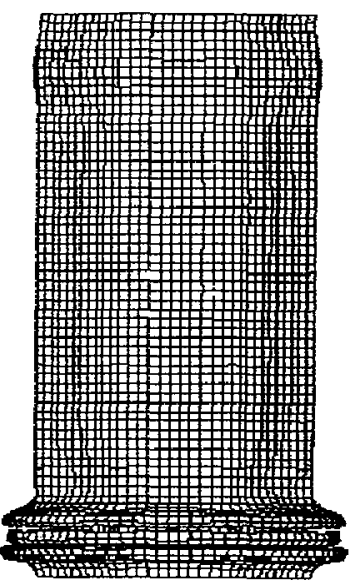

Front liew

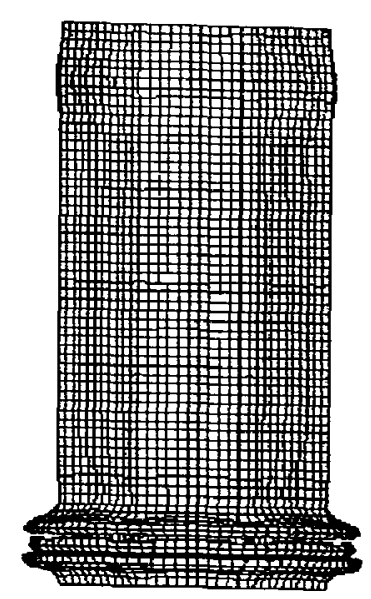

Side I'iew:

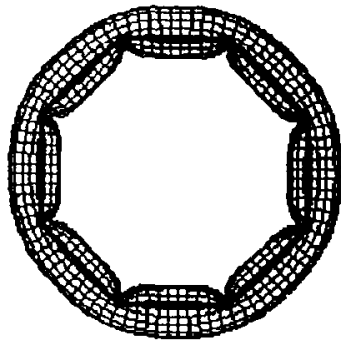

Top lien:

b)
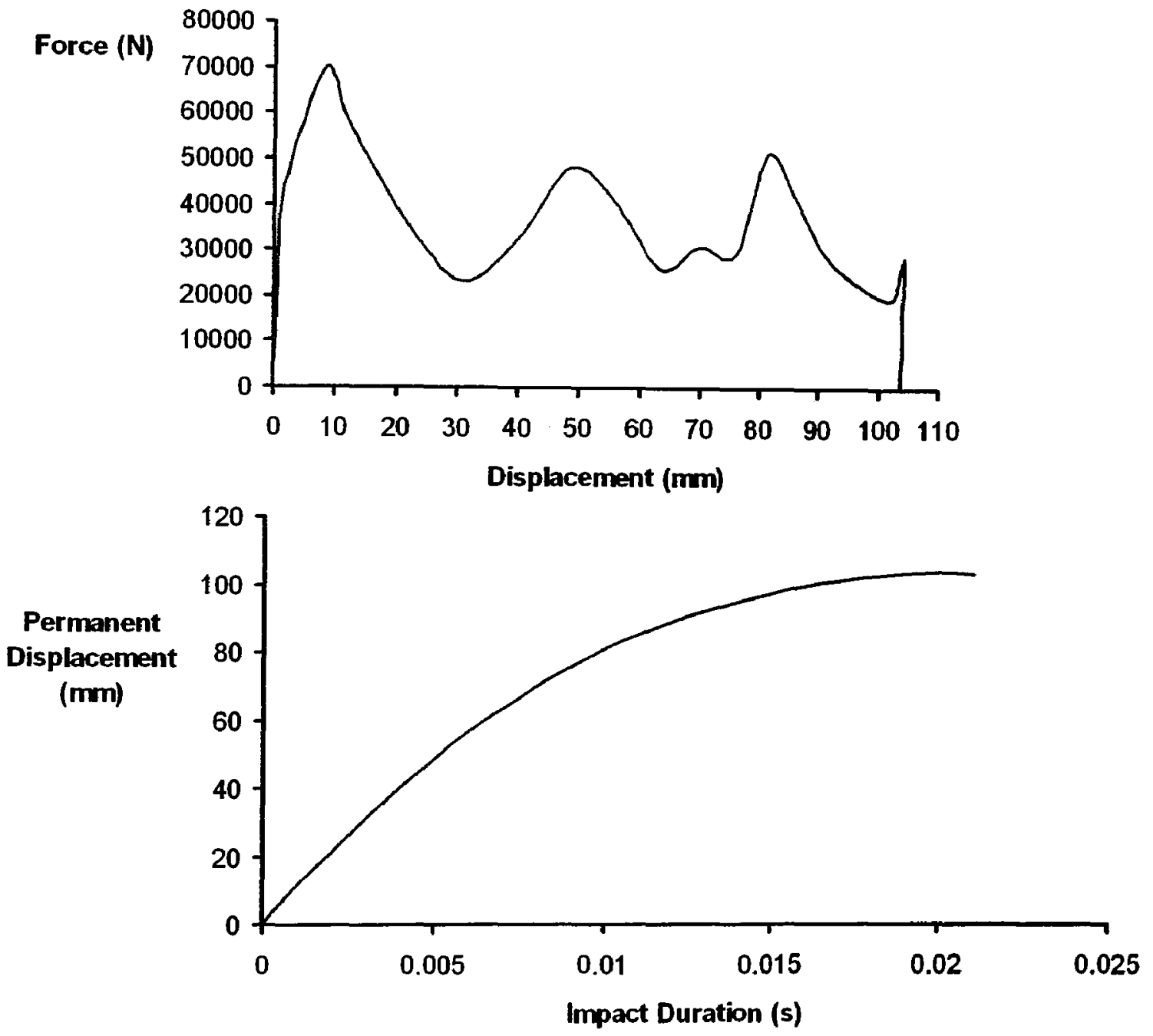

re $A-19$ (a) Final deformation views of LS-DYNA model LS2.0-4-OCT

(b) LS2.0-4-OCT Dynamic Axial Crushing Force vs Permanent Displacement.

(c) LS2.0-4-OCT Permanent Displacement vs Impact Duration. 
(a)

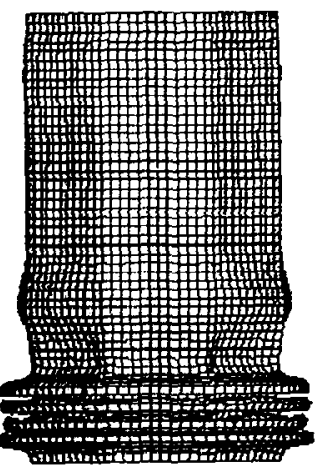

Front rien:

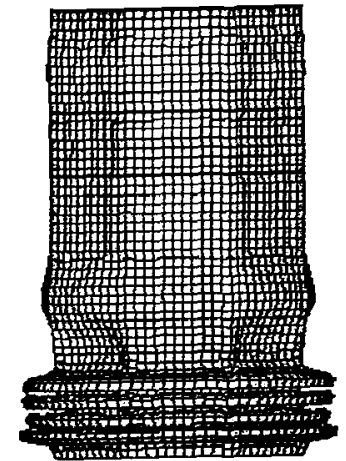

Side View

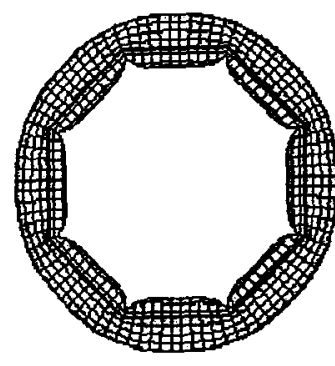

Top Vien:

(b)

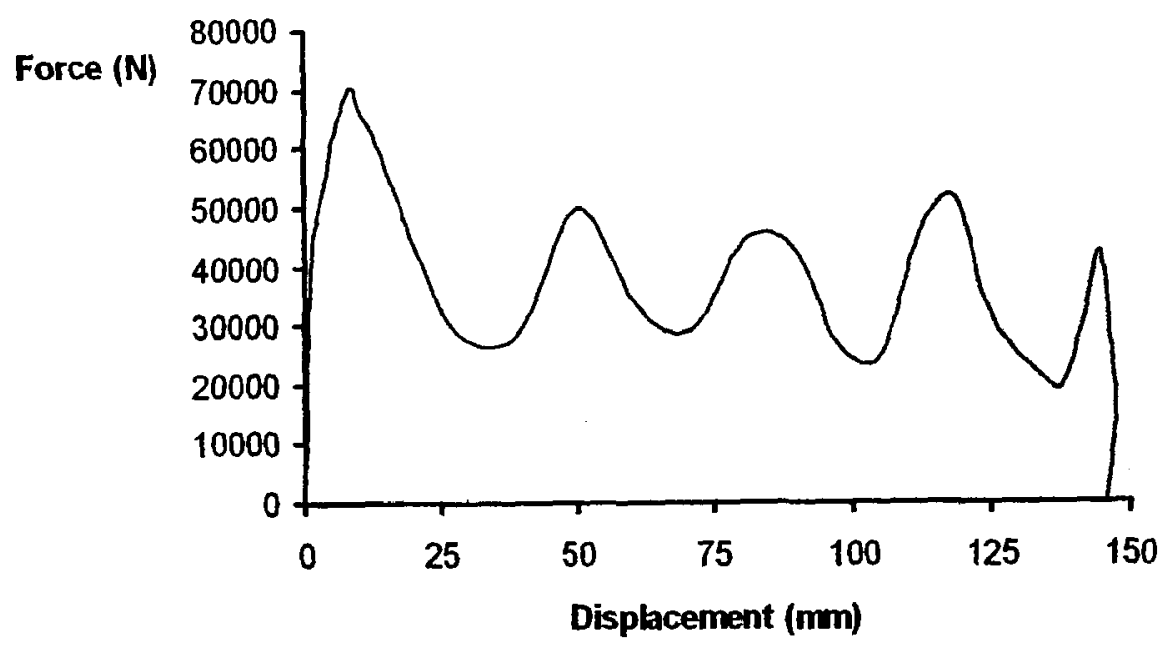

(c)

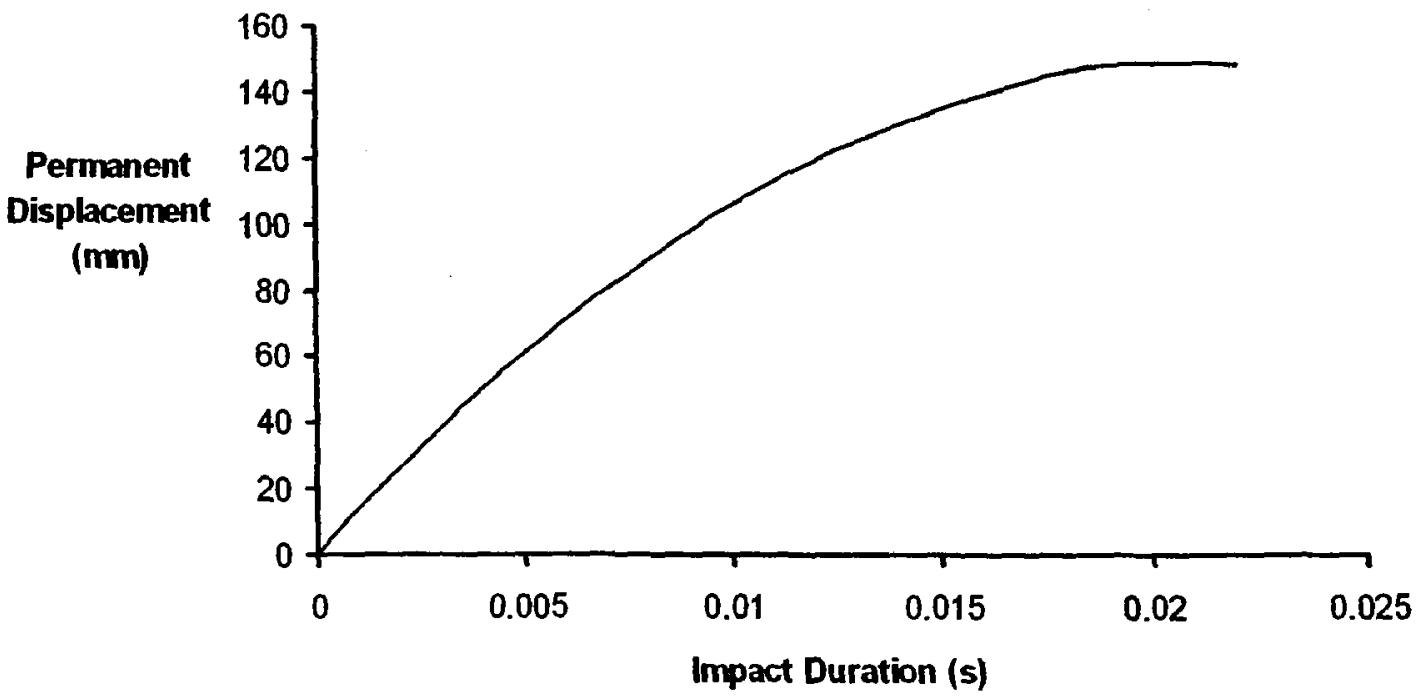

Figure $A-20$ (a) Final deformation views of LS-DYNA model LS2.0-5-OCT.

(b) LS2.0-5-OCT Dynanic Axial Crushing Force vs Permanent Displacement.

(c) LS2 0-5-OCT Permanent Displacement vs Impact Duration. 
)

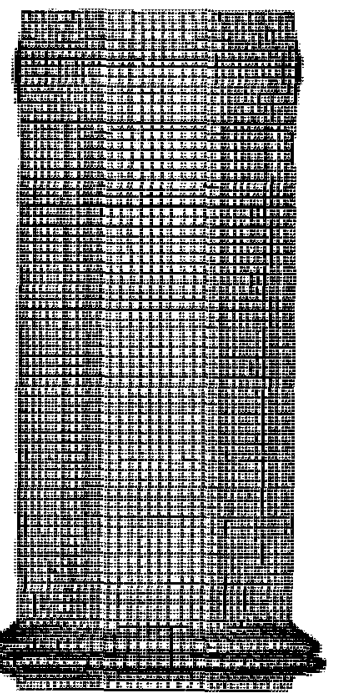

Front trien:

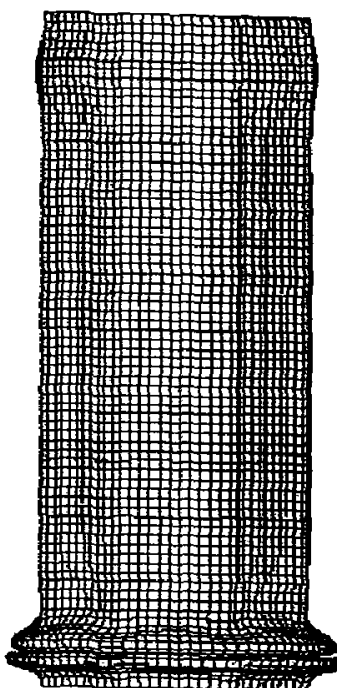

Sicle liew

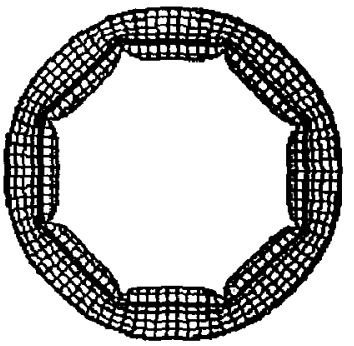

Top lice:

b)
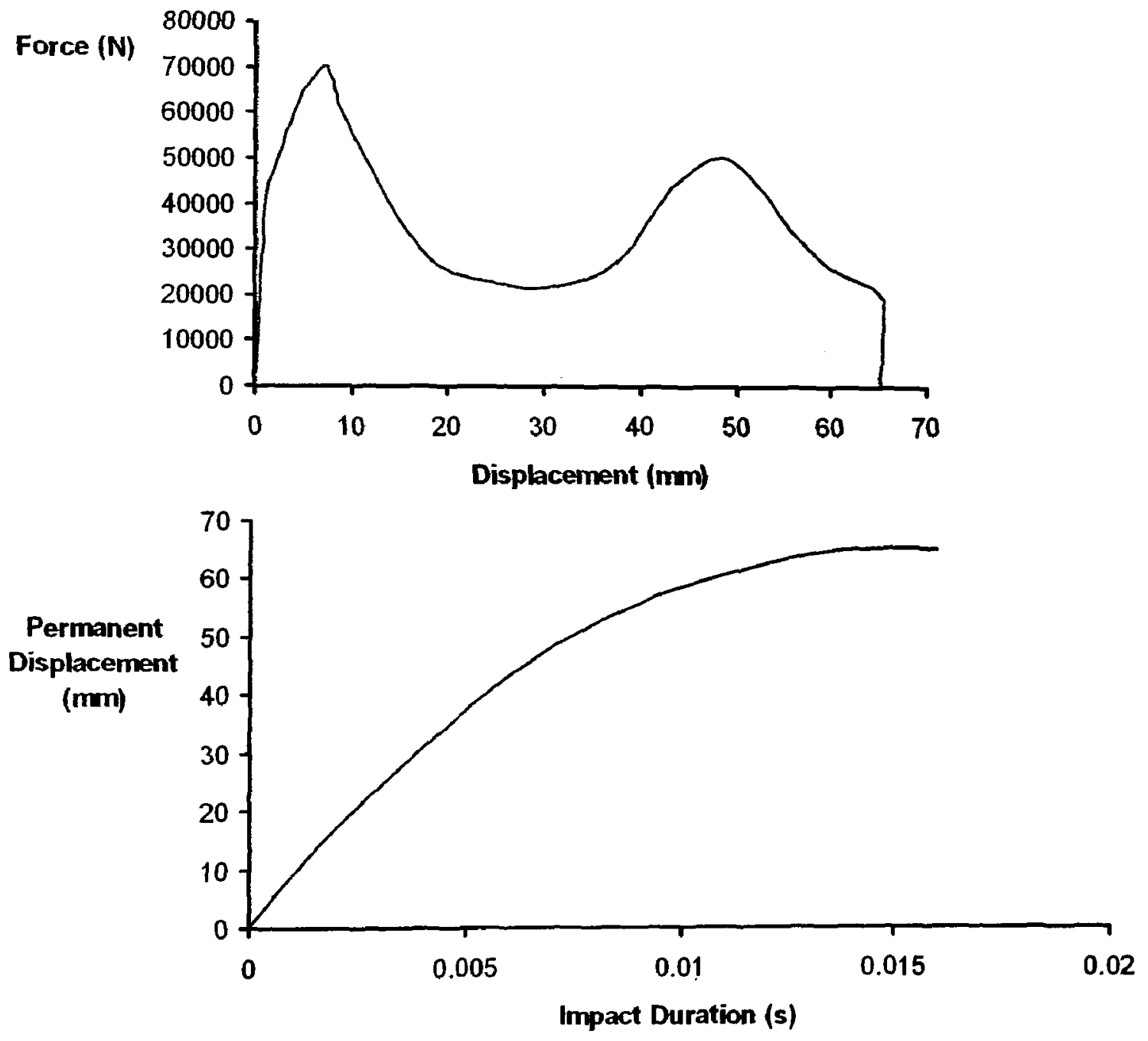

we A-21 (a) Final deformation views of LS-DYNA model LS2.0-6-OCT.

(b) LS2.0-6-OCT Dynamic Axial Crushing Force vs Permanent Displacement.

(c) LS2 0-6-OCT Permanent Displacement vs Impact Duration. 
(a)

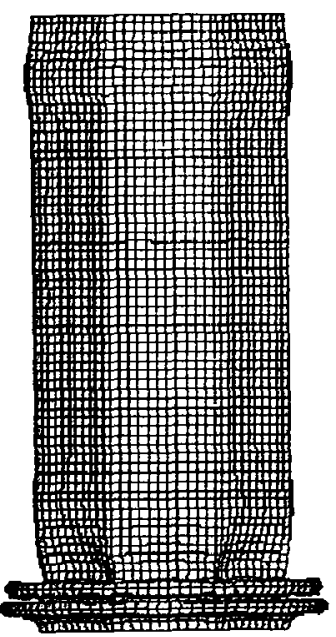

Front liew:

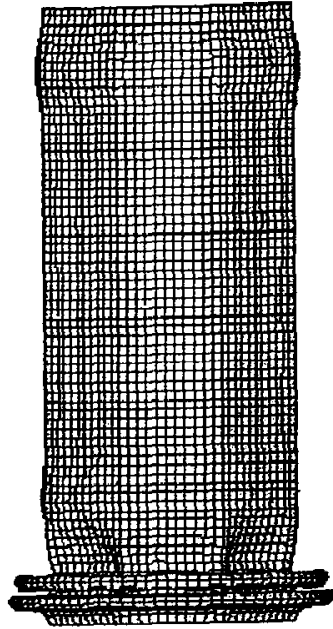

Side View'

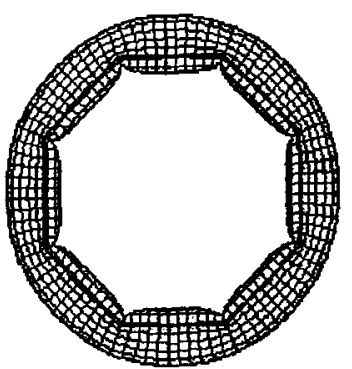

Top View:

(b)

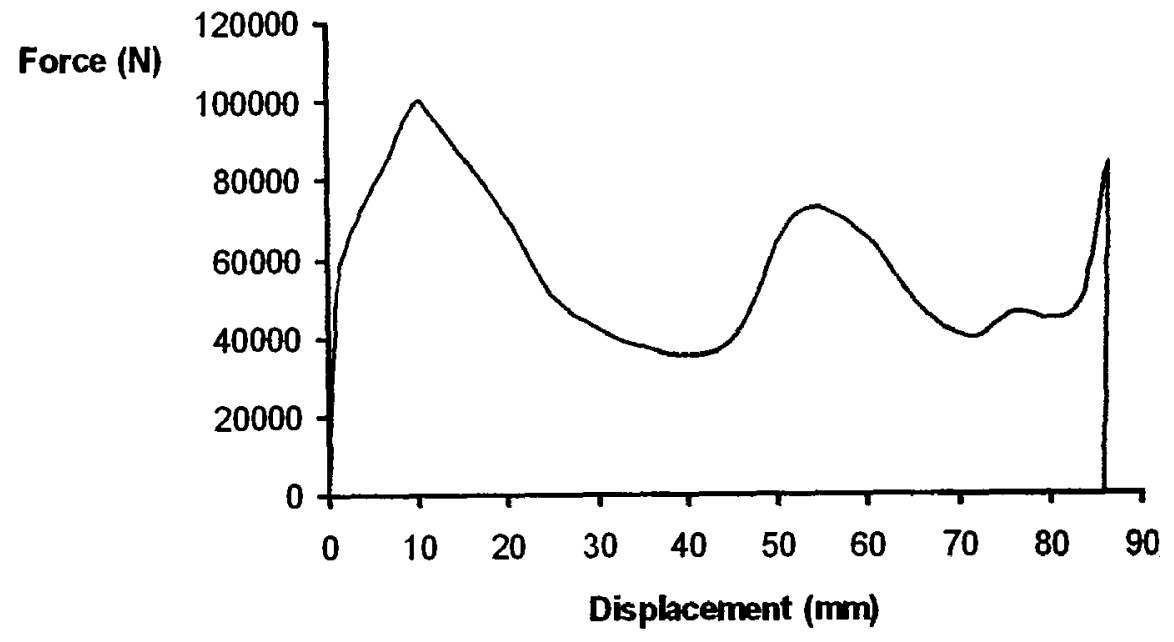

(c)

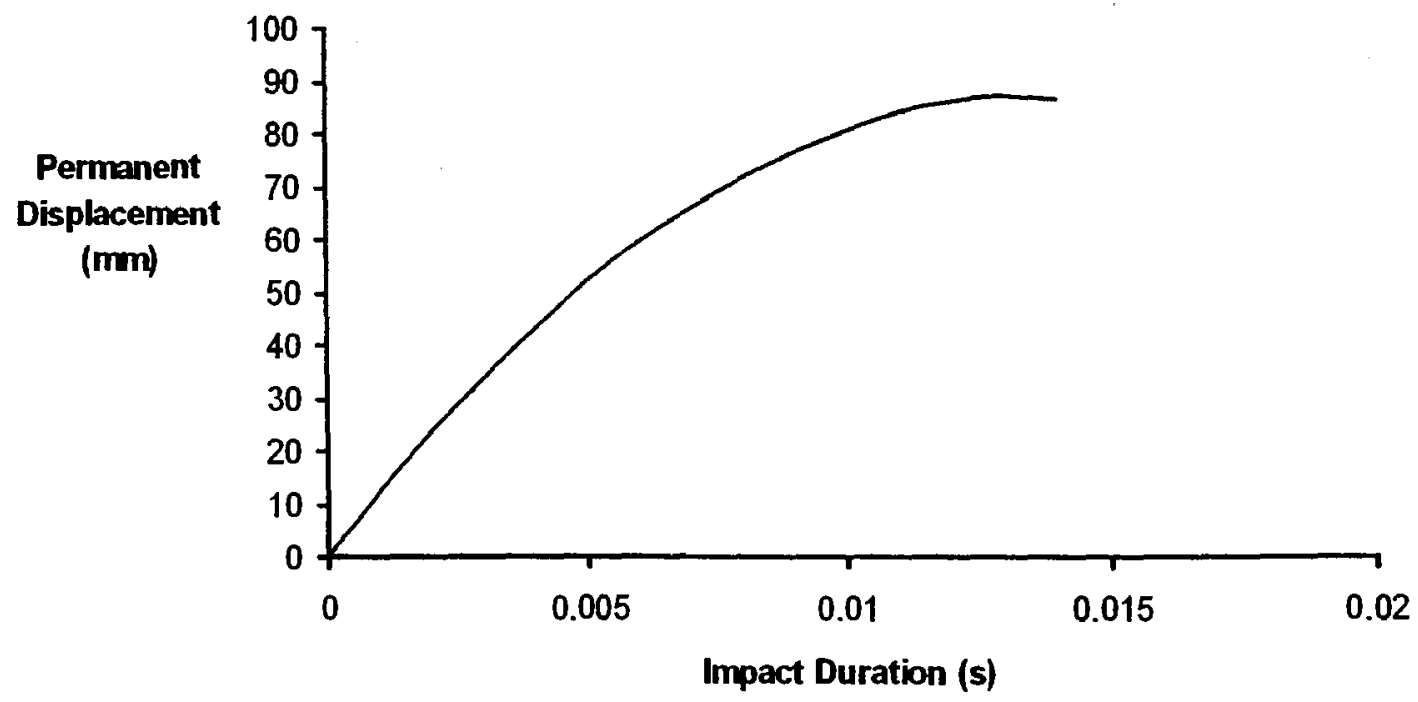

Figure A-22 (a) Final deformation views of LS-DYNA model LS2.5-7-OCT.

(b) LS2.5-7-OCT Dynamic Axial Crushing Force vs Pernanent Displacement.

(c) LS2.5-7-OCT Permanent Displacement vs Impact Duration 
a)

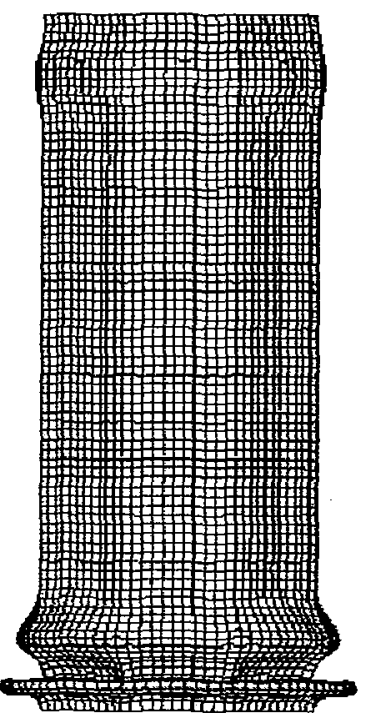

Front liew

(b)
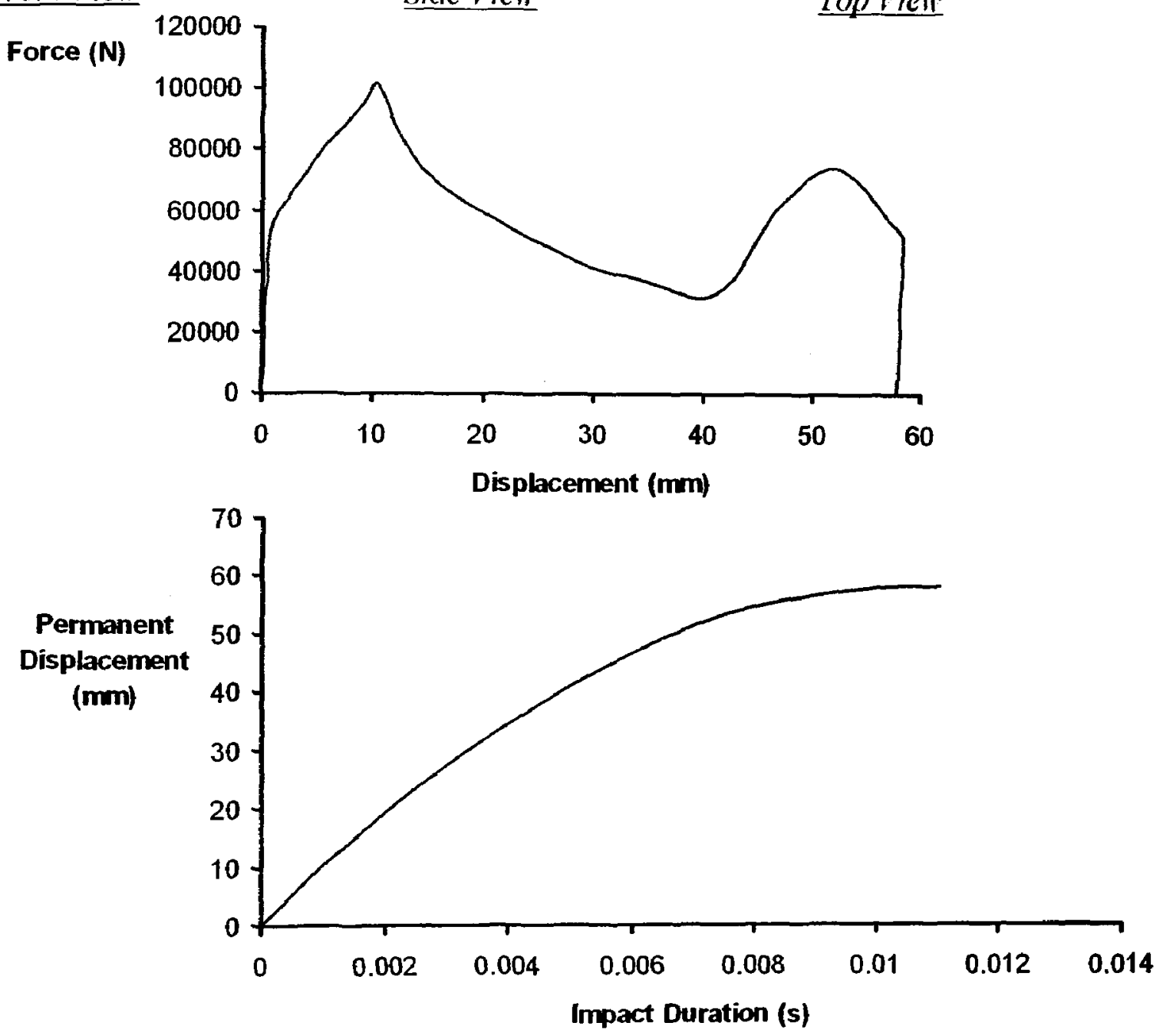

re A-23 (a) Final deformation views of LS-DYNA model LS2 .5-8-OCT.

(b) LS2.5-8-OCT Dynamic Axial Crushing Force vs Permanent

Displacement.

(c) LS2.5-8-OCT Permanent Displacement vs Impact Duration. 
(a)

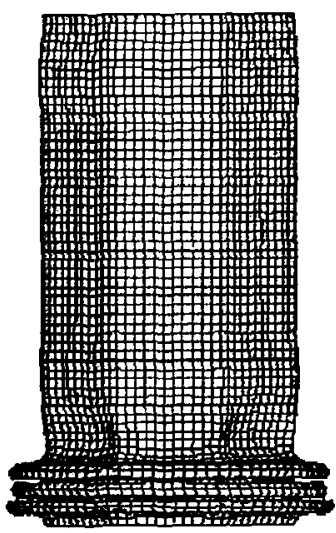

Front Vien

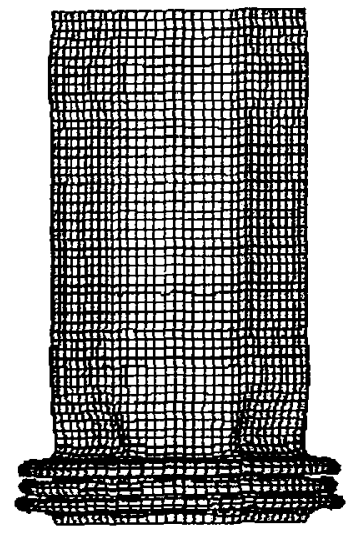

Side liew

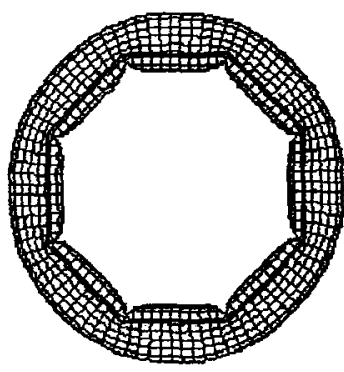

Top Vien'

(b)
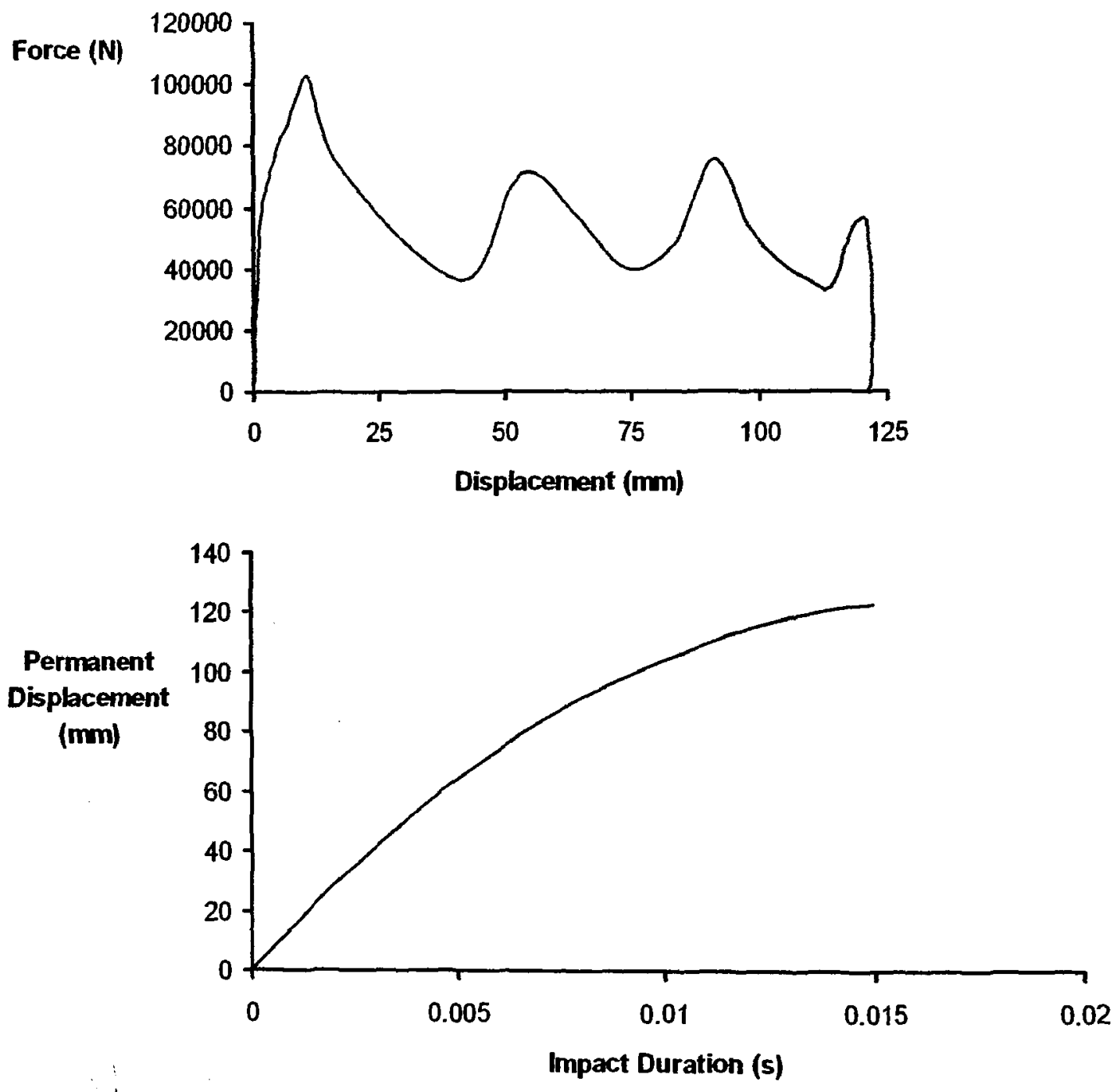

Figure A-2t (a) Final deformation views of LS-DYNA model LS2.5-9-OCT.

(b) LS2.5-9-OCT Dynamic Axial Crushing Force vs Permanent Displacement.

(c) LS2.5-9-OCT Permanent Displacement vs Impact Duration. 